


\section{FAUNA EUROPAA}

\section{MOLLUSCORUM EXTRAMARINORUM.}

\section{PRODR0MUS.}

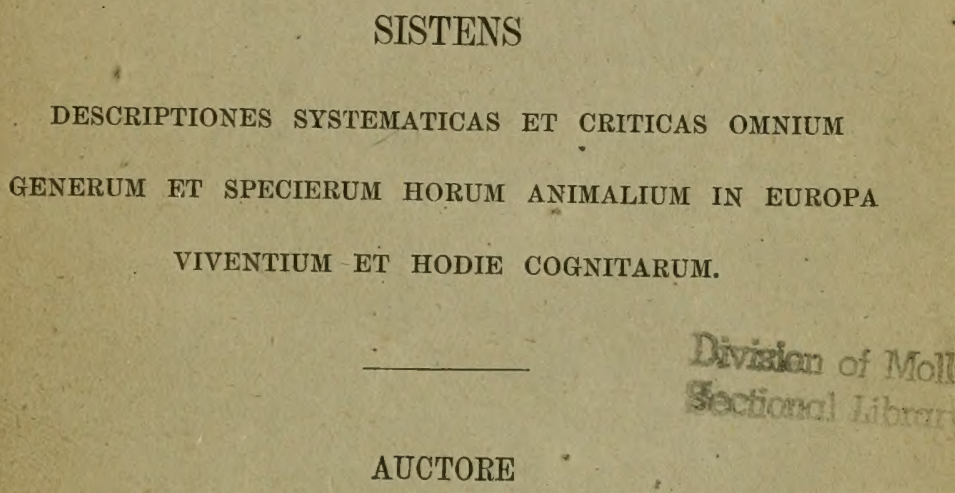

DR. CAROLO AGARDH WESTERLUND.

FASCICULUS I. 



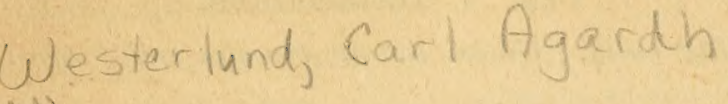

\section{Fauna Europæa \\ Division of Mollusks}

\section{Molluscorum Extramarinorum.}

Prodromus.

\section{Malacozoa}

(Mollusca.)

Animalia vertebris et articulis destituta, corpore gelatinoso, pallio ut plurimum cincto, aut nudo aut testa vestito; circulatione humorum duplici, corde bi-triloculari; organis respirationi peculiaribus, vesicis pulmonaceis vel branchiis; systemate nervorum ganglioso, gangliis circa oesophagum positis.

Cl. I.

\section{Malacozoa Gastropoda Cuvier.}

IMollusca capite libero, tentaculato, oculifero, corpore oblongo, disco longitudinali musculoso repente, testa uniralvi vel. nulla (interna.)

\section{Ordo I.}

\section{Gastropoda Inoperculata.}

Mollusca hermaphrodita, vesica pulmonacea, ostiolo ex arbitrio clausa vel aperta, aërem respirantia; testa nulla externa aut (plerisque) spiralis, semper operculo destituta.

Sect. 1. Geophila FÉr. (Stylommatophora Scнм.)

Moll. pulmonata, terrestria, tentaculis cylindraceis retractilibus quattuor instructa, superioribus duobus majoribus, apice orutiferis (inferioribus interdum inconspicuis).

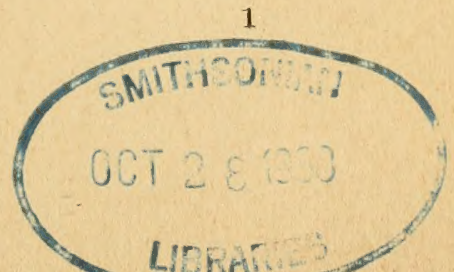




\section{A) Orificium org. gen. externum commune.}

\section{Fam. 1. Testacellidæ.}

Maxilla nulla. Uncini radutie aquales spiniformes.

\section{Gen. Testacella Cuvier.}

Animal limaciforme, parte postico superiore testa obtertum, collo elongato sulcis 2 lateralibus exarato; ani et respirationis orificia in extremitate postica, orificium genitale infra tentaculum majus lateris dextri; uncini radula hamati.

Testa externa, solida, paria, auriformis, apice vix spirata, intus margaritacea; apertura amplissima, ovalis, obliqua, margini columellari depresso. Animal in testa neutiquam contineri protest.

Syn. Testacella Cuvier, 1800. - Helicolimax Férussac, 1801. - Testacellus Faure-Biquet, 1802.

Habitat sub lapidibus vel in terra, sæpe sat profundo, in hortis et fruticetis, ubi tempore nocturno vel de die sub coelo pluvio lumbricis vescitur. Fossilis in str. miocen. et pliocen. medio.

\section{† Testa coniexa.}

1. T. maugei FÉR. (1819): t. ovali-oblonga, virescens $\mathrm{v}$. brunnescens, convexa, deinde rugosa: apertura antice rotundata. postice obtuse angulata; long. 13-17, lat. $7-11$, alt. $3^{1 / 2}-5^{1 / 2}$ mm. (Gall., Hisp., Lusit., Britan.) T. anfr. 2, sutura carinata, columella lata, callosa, non truncata. Syn. T. haliotoides Lam. 1801. T. burdigalensis Gass 1855. T. oceanica Gratel. 1855. Fig. Fér. Hist. t. 8 f. 12. Gass. \& Fisch. Mon. Test. t. 2. f. 1.

2. T. companyoni Dup. (1847): t. ovata, summum alba, reliquum griseo-nigrescens, convexa, striata; apert. antice angustata, postice rotundatior; long. 17, lat. 8, cr. $2^{1 / 2} \mathrm{~mm}$. (Pyren.orient.) $\mathrm{T}$. sat grosse irregul. striata, spiræ rudimento minimo obtuso, columella depressa, antice vix subtruncata. Syn. T. canigonensis Gratel. 1855. Fig. Dup. Moll. Fr. 1. f. 3.

3. T. haliotidea DRP. (1801): t. ovalis, fulvida, tenuis, convexiuscula, rugosa, apice e columella non soluto; apert. antice rotundata et dilatata, postice attenuata; long. 6-10, lat. 4-7, alt. $2 \mathrm{~mm}$. (Gall., Hisp., Lusit, Corsica, Britan.) T. anfr. 11/2, apice fere mediana, columella arcuata, non sinuata, postice attenuata, marg. aperturæ dextro fere verticali, cum colum. angulum formante Fig. Dup. Hist. t. 1. f. 1. 
var. scutulum Sow. (1823): t. angustior, postice valde attenuata, margine dextro ad columellam non angulato, spira productiore. T. anglica Gratel. 1855.

4. T. Pascali Mass. (1870): t. ovata, cornea, crassa, convexo-tectiformis, valide sulcata, apice prominente e columella maxime distante; apert. exacte ovata, superne valde siuato-sulcata; long. 10, lat. $6 \mathrm{~mm}$. (Gall., Haute-Loire.) T. anfr. 2, margine columellari valido, complanato, curvato, superne crassissimo, inferne subtruncato.

5. T. episcia Bourg. (1862): t. ovata, fulva, crassa, convexa, striato-sulcata, apice exiguo e columella maxime separato; apertura ovata; long. 9, lat. $6 \mathrm{~mm}$. (Gall. ad Nizza.) T. anfr. $11{ }_{2}$, columella crassà, fere plana, areuata. Fig. Bourg. Moll. Alp. Mar. t. 1. f. 1-4.

6. T. bourguignati Mass. (1870): t. oblongo-elongata, cornea, parum crassa, parum convexa, striata, apice exiguo e columella parum distante; apert. oblonga; long. $7 \frac{1}{2}$, lat. $4 \frac{1}{2} \mathrm{~mm}$. (Pyren. orient., ut ad Preste.) T. anfr. $\mathbf{1}^{1 / 2}$, colum. sat tenui, planulata, curvata, inferne subtruncato.

\section{ti Testa planulata.}

7. T. bisulcata Risso (1826): t. ovata, ferrugineo-rufescens, tenuissime striata, apice a columella sinu separato; colum. arcuata, antrorsum deflexa; apert. antice angustata; long. 5-7, lat. 3-4, cr. $1-1^{1} / 2 \mathrm{~mm}$. (Gall. merid., Vendée et Bretagne.) T. spiræ rudimento minimo, columella depressa, postice angustata, antice subtruncata. Syn. T. galloprovincialis Gratel. 1855. Fig. Dup. Hist. t. 1. f. 2 .

8. T. drymonia Bourg. (1862): t. trigonalis, cornea, valide striata, apice acuto e columella perarcuata, basi subtruncata separato; long. 7 , lat. $5 \mathrm{~mm}$. (Ital., ins. Capri.) T. anfr. $1 \frac{1}{2}$, apice levi, recurvo.

9. T. beccarii Isset (1868): t. trigonali-ovata, cornea, rugosostriata, apice acuto; columella incurva, elevata; apert. postice acuminata; long. $6 \frac{1}{2}$, lat. 6 , alt. ${ }^{3} / 4 \mathrm{~mm}$. (Ital., Toscana, frequ. ad Firenze.) T. anfr. 11/3, obliqua. Fig. Bull. mal. ital. I. t. 6. f. 1-4.

10. T. pecchioli Bourg. (1861): t. elongata, cornea, rugoso-striatula, apice sicut mammillato; columella parum arcuata, 
antrorsum deflexa; long. 6, lat. $2^{1 / 4} \mathrm{~mm}$. (Ital., frequ. prope Firenze.) T. coaretata, anfr. unico.

11. T. servaini Mass. (1870): t. oblonga, pallide cornea, argute striatula, fere plana, apice minutissimo e columella non distante; apert. ovata; columella curvata, basi attenuata; long. $4^{1} / 2$, lat. $3 \mathrm{~mm}$. (Pyren. orient., ut ad Preste.) T. anfr. 11/2, columella antrorsum deflexa, superne valida, ad basin acuminata.

Obs. Suspicor unam alteramve speciem in specim. junior. aliarum fundatam esse.

\section{Gen. Daudebardia Hartuann.}

Animal subcylindraceum, elongatum, superne longe postice in extrema parte testa munitum, in tergo a tentacul is superioribus sulcis 2 demum divergentibus et 2 aliis lateralibus confuentibus instructum; ani et respirationis orificia in parte postica ad dextrum sub testa, orificium genitale in parte posteriori tentaculi majoris dextri; pallium minimum; radula maxima, uncinis non hamatis.

Testa haliotidea, perforata, depressa, cornea, paucispira, horizontaliter et rapide involuta, anfractu ultimo permagno; apertura amplissima, perobliqua.

Syn. Helix Drp. 1805. - Daudebardia HaRtm. 1821. - Helicophanta FÉr. 1822.

Habitat in locis montosis, humidis et umbrosis, in silvis inter folia putrida, sub lapidibus et in terra. Aestuosis imis diebus diligenter se occultat et tantum autumno, hieme et vere supra terram in conspectum venit. Fossilis in pleistocen. supero.

$\dagger$ Testa aperte perforata $v$. minute umbilicata; anfr. ultimus non angulatus.

1. D. rufa Drp. (1805): t. perforata, anfr. 3, sensim accrescentibus, ultimo elongato; spira mediocris, $1 / 3$ lat. testæ; apertura ovalis, marg. exter. vix arcuato, columell. valde sinuato; long. 5, lat. $3^{1 / 2}$, alt. $1^{1 / 2} \mathrm{~mm}$. (German. merid. \& media, Helvetia.) T. striatula, rufescens. Syn. Vitrina elongata Mandral. (teste Ben.) Fig. Rossm. Icon. f. 39. Hartm. Gaster. t. 3. f. 1-7. Mal. Bl. XIX. t. 2. f. 5-7. (Foss.)

2. D. brevipes Drp. (1805): t. perforata, anfr. $2-2^{1} / 2$ celeriter accrescentibus, ultimo dilatato; spira minutissima, $1 / 4$ lat. 
testæ vix efficiens; apert. rotundata, marg. exter. valde arcuatim extenso, columell. leviter sinuato; long. $4^{1 / 2}$, lat. $3^{1 / 3}$, alt. $1^{1 / 2} \mathrm{~mm}$. (German. merid. \& med., Helv., Ital. bor., Sicil.) T. Iævigata, corneo-virens v. fulva. Syn. Vitrina maravigna Mandral. (teste Ben.) Fig. Hartm. Gaster, t. 4. f. 1-7.

3. D. nivalis Ben. (1847): t. umbilicata; anfr. 3, sensim accrescentes, ultimo peripheria regulariter arcuato; spira magna, $1 / 2$ lat. testæ subefficiens; apert. margine exter. leviter curvato et columell. leviter sinuato; long. $3 \frac{1}{2}$. lat. $21 / 2$. alt. $1 \frac{1}{3} \mathrm{~mm}$. (Sicil. in monte Madonie, Bavaria ad Grossellohe et Schönebach.) T. forma generis Hyalinæ, pallide fulva, apert. latitudine dimidio minor qvam in præcedentibus. Syn.? D. heldii Clessin 1872. Fig. Benoit Illustr. Sicil. t. 1. f. 8. Mal. Bl. XIX t. 2. f. 1, 3, 4.

\section{†† Testa obtecte perforata; anfr. ultimus angulato- depressus.}

4. D. langi Pfr. (1848): t. depressissima, fulva; spira lateralis; apert. ovali-oblonga; long. $6 \frac{1}{2}$, lat. 4 , alt. $1 \frac{1}{3} \mathrm{~mm}$. (Banatus.) T. intus diffuso-callosa, spira minutissima, anfr. 2, columella superne in callum breviter reflexa, sicut in sequentibus.

5. D. transsilvanica Biecz (1859): t. depressa. albida v. fulva, parva; spira terminalis; apert. oblonga; long. $21 / 5-3^{1 / 3}$, lat. $1-1^{2} / 3$, alt. vix $1 \mathrm{~mm}$. (Transsilv. passim, sæpius in silvis fagineis $2-5000^{\prime}$ s. m. positis.) T. anfr. 2, ultimo ovali-oblongo; peristoma cartilagineum.

6. D. sicula Ben., Fisch. (1857): t. valde depressa, succinea; spira lateralis; apert. ovata, marginibus arcuatis; long. $4^{1} / 2$, lat. $2^{3 / 4}$, alt. $1^{1 / 4} \mathrm{~mm}$. (Sicilia.) T. oblonga, anfr. $2^{1 / 2}$, marginibus non parallelis; spira fere plana. Fig. Benoit Illustr. Sic. t. 1.f. 7.

\section{Gen. Glandina Schumacher.}

Animal elongatum, antrorsum angustatum, tentaculis superioribus apice ampulla obliqua ultra oculum prominula terminatis; ad latera oris utrinque lobus trigonus elongatus recurvatus; os superne papillis 3 et in lateribus utrinque 3 munitum; dorsum a tentaculis usque ad testam sulcatum; radula semiovalis, uncinis numerosis in seriebus obliquis.

Testa ovato-oblonga sub epidermide lutescente albida, longitudinaliter plicatostriata, sepius sericea, numqvam splendida, plerumque lineis spiralibus subtilibus decussata; 
anfr. 6-8, ultimo basi attenuato; sutura crenulata; apertura angusta, dimidiam longitudinem requans, columella busi antrorsum arcuata, truncata, peristomate simplici.

Syn. Voluta, Buccini, Bullo, Strombi, Helicis, Bulimi, Achatina etc. sp. Áuctт. Otiacina Borten Mus. 1798 (teste Pfr.) Polyphemus Montw. 1810. Glandina Sснuм. 1817.

Habitat in muscis et sub lapidibus, locis humidis.

1. G. algira BRUG. (1789): t. oblongo-conica, tenuiter striata; anfr. 6-7, ult. convexiusculus, columella subrecta. (Carniol. mer., Istria, Dalm., Ital., Grecia.) T. sutura marginata, minute crenulata; anfr. $6-7$, celeriter accrescentes, ultimus valde descendens; apert. dimidiam longitudinem subæquans, acuminato-oblonga, marg. exteriore stricto, medio antrorsum dilatato; long. 40, diam. 12, apert. long. 19, diam. $7 \mathrm{~mm}$. Variat magnitudine. Syn. Bulimus poireti C. Pfr. 1828. Ach. cornea Brum. 1838. Gl. algira v. angustata Mart. Mal. Bl. VI. p. 162. Fig. Rossm. Icon. f. 123. Pfr. Nat. III. t. 7. f. 3,4 .

2. G. dilatata Z.: t. ovato-conica, confertim costulato-striata; anfr. 6-7, ult. convexus; columella valde arcuata. (Sicitia, Corfu.) T. sutura albo-marginata, crenulata, anfr. 6-7, apert. dimidiam longitudinem superans, ovalis, subtus dilatata, marg. exter. arcuato; long. 36, diam. 14, ap. long. 20, lat. $9 \mathrm{~mm}$. Syn. Polyph. tumidus Villa. Fig. Kust. Mon. t. 17 f. 19-21.

3. G. compressa Mouss. (1859): t. oblongo-cylindracea, confertim costulato-striata; anfr. 5, ult. subimpressus; columella subrecta. (Corfu, Cephalonia et Epirus.) T. sutura submarginata, irregulariter crenulata, apertura acute piriformis, dimidiam longitudinem æquans, marg. exter. in tertia parte antrorsum convexo; long. 35 , diam. 11, ap. long. $16 \mathrm{~mm}$.

4. G. delesserti BourG. (18-): t. ovato-ventricosa, confertim costulato-striata; anfr. 4; columella arcuata. (Corcyra.) T. fusco-cornea, sutura argutissime costulata v. granuloso-marginata, apertura elliptico-oblonga, angusta, $3 / 4$ longitudinis æquans; long. 9 , diam. 5, ap. long. $7 \mathrm{~mm}$.

\section{Fam. 2. Limacidæ.}

Maxilla lavis. Uncini radule incequales, medii tricuspidati, breves, laterales elongati, hamiformes.

Subfam. Limacina. Corpus per totam longitudinem cum pede conjunctum, dorso carinatum, orificio respira- 
tionis postico; forea caudalis nulla. Testa unguiformis, interna a clypeo obtecta.

\section{Gen. Limax Lister.}

Animal dorso postice carinatum, clypeo concentrice striato, postice plus minus angulato vel rotundato; maxilla in medio rostriformiter producta; uncini vadula acuti, medii tricuspidati $v$. utrinque tuberculati; laterales simplices $v$. denticulo postico.

T'esta interna tenuissima, margine membranacea, nucleo laterali.

Syn. Limax Lister 1678, Lin. 1758, MülLer 1774, pr. p.

Habitat in hortis, fruticetis et campis, in truncis et radicibus arborum putridis, inter folia humida vel putrida et sub lapidibus, præcipue in densis et umbrosis silvis. Fossilis in str. miocenico et pleistocen. medio et supero.

A) Clypeus corpore cetero multo brevior.

$广$ Clypeus postice medio elongatus, sapius acute angulatus. (Species sapissime maximce, 10-13-18 usque ad 36 centimet.)

*) Carina abdominalis albida (raro nigra).

๔) Ruga undique colorate, dorsales sulcis angustis disjuncte.

a.) Solea pedis semper fere bicolorata, clypens obscurus, unicolor.

1. L. maximus LiN. (1758): squamæ corporis magnæ, oblongæ, strictæ, tentacula crasse granosa; long. 150-180, lat. 14-18 mm. (Europa.) Cineero-niger; abdomen obsolete longitudinaliter 4 fasciatum, dorso carina validissima, acuta, albida, solea partibus exter. cinereis. Var. 1. niger, unicolor, solea obsolete bicolorata; 2. albus Paasch, unicolor; 3. fasciatus: cinereus, fasciis 5 , latis, nigris, undulatis; 4 luctuosus M. T.: ater, carina pallida; 5. flavescens, unicolor, solea obsolete bicolorata; 6 cinereo-nebulosus Malm: albicans, elypeo flavido, ab domine postice maculato, solea tota albidia. 7. calosoma Eis. \& Stuxb obscure otivacens, maculis pallidioribus, clypeo atro, marginibus pal lidis \&t. Syn. L. cinereo-niger Wolf (1822). Gen. Eutimax M. F,, Malm (uncinus radulæe medius simplex, laterales apiculo secundario), subgen. Heynemannia (clypeus postice elongatus, sudor non coloratus.) 
2. L. nubigenus Borra. (1863): differt a pracedente corpore elongatissimo, cylindrico-fusiformi, angusto (lat. tantum 5-6 mm.) nigro, carina et zonula mediana soleæ albidis et collo cinereo. (Pyrenca altior.)

b) Solea pedis unicolor, alba.

aа) Clypeus nigro-maculatus.

3. L. cinereus Lister (1673): squanæe corporis angustæ, undulato-flexuosæe, tentacula minute granulosa; long. 150-180, lat. $20 \mathrm{~mm}$. (Europa occid.) Cinereus; clypeus postice longe rostratus, abdomen fasciis longitudinalibus vel maculis nigris in seriebus positis, dorso carina alba obtusa. Syn. L. maximus Auct. plur. (non Lin.), L. antiqvorum Fér. 1819. Gen. Eulimax subg. Heynemannia Malm. (Foss.)

bb) Clypeus non nigro-maculatus.

4. L. unicolor HeYnem. (1862): squamæ corporis angustissimæ, valde flexuosæ, striæ concentricæ clypei tenuissimæ. (German. ad Frankfurt am $M$. et Hanau, Dania ad Frederitisdal; Norv. ad Bergen?) Cinereus, clypeo immaculato. Forma norvegica atra, abdomine fasciis $\mathbf{5}$ pallidis, clypeo albomaculato, postice perbreve rostrato, squamis ita flexuosis, ut corpus forte nodulosum videatur. L. cinereo proximus.

5. L. engadinensis HeYnem. (1862): squamæ corporis et strix concentrica clypei validx. (Alpes, in S:t Moritz, Helvetia, et Transsiliania.) Cinereus, clypeo immaculato. L. cinereo proximus.

6. L. companyoni Bourg. (1863): luteus, cinereo-variegatus, squamis corporis validis, clypeo magno, concentrice granulosostriatulo, postice valide rostrato; long. 100-120 mm. (Pyrencec orient., ad Collioure et Port-Vendrees.)

7. L. eubalius Boukg. (1864): corpus mediocre, in adversum percrassum, in aversum perangustum, albidum. maculis numerosis flavidis ornatum. (Alpes Gall., circe Grand-Chartreuse.) Fig. Bourg. Mal. Gro-Chartr, t, 1 f. $5-8$.

B) Rugarum partes summa tantum colorate, ruge dorsules sulcis latis disjuncta.

8. L. schwabi FRFLD (1865): striæ concentricæ clypei creberrima, a centro ad marginem anteriorem c. 50 ; squama abdominis altæ, paullulum flexuosæ; carina evanescens. (In montibus Carpathicis.) Animal sæpius ultramarinum v. subviolaceum, interdum indicum, cinereum vel virescens, solea pedis etiam medio atra. 
9. L. transsilvanicus HEYNEM. (1862): striæ concentricæ clypei distanter positæ, a centro ad marginem anteriorem c. 25; squamæ abdominis angustr, rectæ, depressæ; carina alta, longa. (Transsilvania.) Animal colore varium: albidum, carneum, flavidum, cinereum, nigrescens, in latere utroque fascia obscuro, solea pedis medio alba, partibus exterioribus nigro-punctatis.

**) Carina abdominis rubro-sanguinea.

10. L. doriæ Boura. (1861): corpus giganteum, sæpissime aterrimum, solea atra, medio obscure albida, clypeo aterrimo, subgranuloso, postice minime rostrato. (Gallia merid.orient., Ital. bor.) Animal maximum hujus generis (long. usque ad $360 \mathrm{~mm}$.), squamis abdominis validis, carina maxima. Fig. Bourg. Rev. zool. 1861. t. 8 f. $1-11$.

11. L. dacampi Menec.: corpus sordide albescens, lateriluus rufo-fuscis, fasciis 5 decrescentibus sanguineis ac nigris notatum, clypeo carneo-cupreo, concentrice crispato-sulcato, postice cuneato. (Italic bor.) Animal tentaculis cinereis, granulosis, basi latis, clypeo marginibus anticis carneis, solea cinereo-nigrescente marginata.

12. L. callichrous Bourk. (1861): corpus luteolum, utrinque zonis duabus nigerrimis ac utrinque ad pedis marginem nnaculis nigris interruptis preditum, clypeo postice rostrato, concentrice striatulo, aterrimo ac maculis luteo-vinosis ornato; long. 60 mm. (Gall., Alp. Marit.) Animal solea flavida, ad margines paullo fusca.

13. L. erythrus BovkG. (1864): corpus rufum, unicolor, clypeo nigro-maculoso, postice acuto. (Gall., Alpes ad Grande Chartreuse.) Fig. Bourg. Mal. Grande Chart. t. 2 f. 1-8.

14. L. bielzi Seibert (1873): corpus carneum, interdum dorso et lateribus 4 fasciis nigrescentibus ornatum, clypeo obscure rufescente vel atro, postice longe rostrato; long. ad $140 \mathrm{~mm}$. (In montibus Carpath.) Animal sqvamis abdominis angustis, paullulum undulatis, remotis, solea pedis bicolorata, muco dorsi et clypei rufo, pedis non colorato.

if Clypeus postice medio obtuse angulatus.

*) Species majores (long. 70-90-100 mm.)

a) Clypeus maculatus. (Solea luteola.)

15. L. variegatus DRP. (1801): flavidus; abdomen maculis 
nunerosis, pallide brunneo-cinereis, clypeus flavus, maculis parvis brunneo-cinereis marmoratus. (Europa, excl. parte bor.) Animal ad $100 \mathrm{~mm}$. longum, muco luteo, carina albida, undnlata, acuta preditum. Syn. L. flavus Jeffr. (1862); Eulimax (Plepticolimax) fluvus Mlalm (1868). Fig. Mal. Bl. X. t. 3 f. 5. In cellis penariis et in puteis hree species vivit.

16. L. psarus BourG. (1861): cinereo-coerulescens, in lateribus inferne coeruleo-luteus, ubique maculis aterrimis orinatus. (Lomburdie, circa Brianza.) Animal postice acute ac albidulo carinatum, $70 \mathrm{~mm}$. longum.

ß) Clypeus immaculatus.

a) Clypeus striis concentricis usque ad marginem anteriorem productis.

17. L. corsicus M. T. (1855): flavido-griseus, lateribus inferne rufescentibus, clypeo pallide flavido, solea pedis partibus exterioribus rufobrunneis, medio albida. (Corsica et Sardinia.) Animal 9-13 centimet. longum, 15-20 mm. latum, oblongum, rugositate dorsali tenui. Fig. M. T. Hist. moll. t. 3. f. 10-13.

18. L. martinianus Bourg. (1869): Havido-albus, maculis brunneis numerosis sicut translucentibus et in latere utroque fascia pallide brunnea ornatus; solea albida unicolor. (Gallia in Alp). II (aritim.) Animal minus, subcylindricum, rugositate dorsali vix conspicuo.

b) Clypeus parte anteriore transterse striatus, posteriore striis arcuato-incolutis, non concentricis.

19. L. maurelianus Bovrg. (1969): flavidus, fasciis 5 atris longitudinalibus, una dorsali; solea albida. (Gallia in Alp. MLaritim.) Animal hujus sect. minimum, 50-60 mm. longum.

**) Species minores $(30-50 \mathrm{~mm}$.). Corpus molle, sudor uberrimus, lacteus.

20. L. agrestis LIN. (1758): animal variis modis coloratum, clypeo postice brevissime angulato. (Europa.) Corpus postice angustatum, elongatum, sat valide albo-carinatum, solea flavescente; testa depressissima, tenuissima, rectangularis; uncini laterales subsimplices. (Foss.) Variat 1) albidus, immaculatus; 2) punctatus, albidus, atomis nigris sparsis et utrinque linea uigra:.3) filans Hoy, albus, clypeo flavescente; 4) reticulatus Müll., fuscus, clypeo punctis, abdomine lineolis nigris; 5) veranyanus Bourg.: griseus, maculis nigris confertis 
variegatus; 6) tristis M. T., (L. saxorum Baud.) brunneus, clypeo utrinque fasciato: 7) obscurus M. T., subrufescens, obsolete variegatus, tentaculis fuscis et fasciis brunneis; 8) succineus Müll., supra subrufus, subtus albus; 9) varians, griseus, nigro-maculatus et variegatus; 10) lilacinus M. T., lilacinus, unicolor. \&e.

- var. norvegicus WesterL. (1870): postice validissime carinatus, parum angustatus, supra rufescenti-cinereus, subtus cinereo-cretaceus, medio subpiceus, sudore parco; testa crassa, convexa, latere dextro sinuata; uncini later. bicuspidati; long. 11-13 min. (Norv. in Ringerige.)

- var. sylvaticus Drp. (1805): gracilis, paullo major, clypeo violaceo, rufescente, dorso violaceo coerulescente, nec fasciis nec maculis ornatus, limbo luteo non transverse striato. (Gallia merid.)

- var. ctruscus ISSEL (1868): brunneus, interdum pallide inaculosus, carina nigra, valida, clypeo postice arcuato, "sulcis duobus convergentibus notato," solea luteolo-fusca; testa parvula, subquadrata; long. $31 \mathrm{~mm}$. (Ital., Toscana.)

tti Clypeus postice regulariter rotundatus. Corpus mollissimum, mucor luteus. Species parva, 30-60 mm.

21. I. tenēllus Nilss. (1822): luteo-cinerascens, clypeo luteo, tentaculis nigris, fascia in latere utroque obscura; long. 30-60 mm. (Scandin., Brit., Belg., Gall. merid., Germ.) Animal rugis dorsalibus in seriebus regularibus positis notatum; radula $4 \mathrm{~mm}$. longa, $3 \mathrm{~mm}$. lata, seriebus uncinarum long. 135, transvers. 131, uncinis later. bifidis. Syn. L. cereus Held. L. cinctus Heynem. \& plur.

- var. xanthius Bourg. (1866): animal uniformiter luteolo- vel subviridulo-aurantiacum, rugis dorsalibus argutis, elongatis, dorso convexo, ad caudam acute carinato, clypeo valde anteriori, maximo, eleganter striatulo; long. $45 \mathrm{~mm}$. (Germ., Malberg prope Ems.)

- var. squummatinus Moré. (1845): aureo-virescens, lateribus coerulescentibus, tentaculis nigris, quadrofasciatus, lineis lateralibus parallelis, dorsalibus in unum convergentibus. (Lusitania.)

22. L. majoricensis Hermem. (1862): radula $6 \mathrm{~mm}$. longa, $11 / 2$ mm. lata, seriebus uncinarum long. 80, transv. $100 \mathrm{~mm}$.; testa $3 \frac{1}{2} \mathrm{~mm}$. longa, supra 2, infra $1 \frac{1}{2} \mathrm{~mm}$. (Mallorka.) Notis allatis anatomicis mihi tantum cognitum animal. 
B) Clypeus corpori cetero subaqualis, ut in N:o 19 striatus. Species minime, $10-20 \mathrm{~mm}$. longa, subcylindiacer.

23. L. lævis MüLL. (1774): gracilis, vix carinatus, postice brevissime acuminatus, pallide $\mathrm{v}$. sæpius obscure brunneus; long. 15-20 (25) mm. (Europe media et bor., in locis paludosis.) Animal clypeo utrinque rotundato, antice et postice inæqualiter' striato, solea pallide brunnea, muco non colorato, uncinis radula lateral. apiculo secundario. Fig. Mal. Bl. XIX p. 148. Syn. L. brunncus Drp. 1801. L. parvulus Norm. 1852. Li. arenarius Gass. 1859. Krynickillus brunneus, lavis et bourguignati Mab.

- var. mucronatus WEST.: luteo-brunneus, lateribus pallidis, solea alba, clypeo processu conico; long. $10 \mathrm{~mm}$. (Suec. ad Ronneby.)

24. I. heydeni Hexnem. (1863): breviter carinatus, albidus, lutescens v. rufescens, tentaculis brumneis et muco lacteo; long. $10 \mathrm{~mm}$. (S:t Moritz in Ober-Engadin; Dania?) Animal uncinis radulie lateralibus simplicibus.

? L. lombricoides Morku. (1845): "gracilis, carnicolor, atomis nigris notatus, corpore subcarinato, postice acnminato, r'ugosiusculo, clypeo striis adversis sulcato, anterius libero, valde contractili." (Lusitania ad Monchique.) "Une variété plus foncée habite les montagnes de Braga."

Species mihi ignota: L. cristatus KaL., Tauria, - L. cymiacus Mab., Corsica, - L. dymezewiczi Kalmn., Tauria, L. flavidus Fér., Alpes, Pyren., - L. niger MALz., Belgia, L. pallidus Scrr., Liflandia, - L. laygetos Desh., Morea, L. umbrosus Pnic.. Sicilia.

\section{Gen. Lehmannia Heynem.}

Animal ut Limax, sed uncinis radule obtusis, mediis simplicibus, lateralibus emarginatis, limbo coipusculis $1-5$ unciniformibus minutis, maxilla magna, margine anteriore medio late subproducto. Corpus mollissimum, sudor non rolmatus, solea albida v. cinerascens.

Testa interna subrectangularis, angulis obtusis, consistentia valde incqualis: tenuis, membranacea, gramulis calcareis passim conspersa, vel crussa, calcarca, supra convexu, subtus concave, sapius 5-6 mm. longa, $2^{1} \frac{1}{2}-3 \mathrm{~mm}$. lalu, interdim, ex. gr. in Gotlandia, I mm. longa, $6 \mathrm{~mm}$. lata, $5 \mathrm{~mm}$. crassa. 
Syn. Limax Aucot. - Lehmannia Heynemann, 1863.

Habitat in silvis frondosis humidis, ad rivulos et fontes, in arboribus, sub lapidibus et cortice truncorum, inter folia putrida et in muscis.

1. L. marginata MüLL. (1774): coerulescens v. cinereus, aquosus, subpellucidus, carina abdominis alba, acuta, utrinque fascia obscura marginata, clypeo utrinque striga obscura albomarginata notato; long. 50-75 $\mathrm{mm}$. (Europa.)

Variat (in Belgia) rosea Boeck, 1870 (rosea, dorso brunneorufescente, carina pallide rosea, fasciis nigris, clypeo sub lente maculis ovalibus dense ornato) et colorate Boeck, 1870 (aquosa, subpellucida, carina alba subnigro-marginita, clypeo ut in $\alpha$ fasciata, abdomine utrinque brumneo-fasciato), et (in Würtembergia) tigrina Weinl. (pallide virescenti-flavida, clypeo maculis nigris in seriebus 5 ornatis, corpore utrinque fasciis duobus abruptis,) \& flara Weinl. (viridiflava, unicolor.) Fig. Malm. in Göteb. IIandl.' 1868, Lehm. Schn. Stett. t. 4 \& 8 f. 4. Syn. L. arborum Bouch, 1838, L. sylvaticus Goldf., 1856, L. salicinus Bonill., L. scandens Norm. 1852, I. livonicus Schr., L. limbatus Held., L. helveticus Bourg.

- var. heynemanni. BieLz.: alba, clypeo picto, dorso maculis parvis nigris, rugis majoribus et carina alta notato. (Transsilv.)

\section{Gen. Amalia Moq. TAnd.}

Animal dorso toto albo carinatum, clypeo granulosa, postice marginato et illinc sulco circulari in medio sicut bipartito; maxilla antice emarginata et dentate; uncini radula medii tricuspidati laterales secundi ordinis laminibus transverse bipartitis et lamina utraque dentifera.

Testa interna ovalis, calcarea, crassa, margine non membranaceo, mucleo centrali.

Syn. Limax Aucct. - Limax Subgen. Amalia Moq. Tand. 1856. - Amalia Heynemann 1861. - Milax Gray, 1855 .

Habitat in regionibus calcareis, sub lapidibus, in muris calce illinitis antiquis etc. Fossilis in str. miocen. medio et supero.

1. A. marginata DRP. (1805): rufescenti-griseus vel brunneorufescens, punctis et maculis nigris obtectus, clypeo postice late emarginato, utrinque nigro-unifasciato; long. $80-100 \mathrm{~mm}$. (Hisp., Gall., Belg., Brit., German. media, merid. et occid., Transile., Sicil., Ital., Helr., Tirol., Russia merid.) Animal sub- 
cylindricum rubro-cinereum, flavescenti, carinatum, subtus albidum, dorso oblique sulcato-granulatum. Syn. $L$, souerbyi Fér. 1823. $L$. carinatus Leach ap. Gray 1852. L. argillaceus Gass. 1856. Milax marginatus, carinatus, sowerbyi et pyrrichus Mal). 1870. Fig. M. Tand. Hist. t. 2 f. $4-17$.

2. A. gagates Drr. (1801): obscurus, clypeo gibboso, bilobato, efasciato; long. $70-90 \mathrm{~mm}$. (Gall., Hisp., Lusitan., Itulia, Siril., Brit.) Animal subcylindricum, rufescenti-nigrum, interdum plus minus plumbeum v. olivaceum, acute carinatum, suhtus pallichum.

Speries mihi ignota: A. atrata MaB. (L. agrestis Morel.). Lusitania.

Sulfam. Vitrinina. Corporis pars posterin a pede distincta, spivaliter contcrente ef testa restila. - Testa lemis, nitidn, sepuissime dejmessa, grevistomate temui. vecto et aruto.

\section{Gen. Parmacella Cuvier.}

Animal elongatum, subcylinitraceum, postice valide ravinutum, forea caudati mulla; clypeus magnus, pone medimm dorsi positus, minute granulosus; maxilla lavis, margine edentato medio vix producto; orificium respirationis postirum. IIucus albus glutinosus.

T'esta rudimentaris, pars una (anterior) interna, sutovatis a clypeo obtecta, pars altera (posterior) externa minima, spiralis, dextrorsa, colorato, columella depressa, apertura amplissima.

Syn. Parmacella Cuvier, 1864.

Habitat in pascuis aridis Gallix meridionalis, in fruticetis Quercus cocciferæ. Animal nocturnum.

1. P. valencienni WeBis. et v. Bex. (1836): animal supra adumbratione griseo-flavescenti, cervice cinereo-flavescenti, clypeo subaurantiaco, maculis nigris inæqualibus ubique munito; testa margine dextro superne depresso; long. anim. 90, lat. $20 \mathrm{~mm}$. (Lusitania.)

2. P. moquini Bourg. (1860): animal colore testaceum, unicolor, testa margine dexto non depresso, parte anteriore tenui, pellucida, elliptica, antice cartilaginea, sextuplo majore quam testa externa. (Gallia ad Crau prope Arles.) Animal precedenti magnitudine æquale, sed minus carinatum, testa flavido-virescens, minor, $17 \mathrm{~mm}$. longa, $10 \mathrm{~mm}$. lata, $2 \frac{1}{2} \mathrm{~mm}$. alta, interna difficillime liberanda. Syn. P. valencienni M. 'T. Hist. II. p. 35. t. 4 f. 9-18 (non Webb. \& v. Ben., teste Bourg.) 
3. P. gervaisi M. TAND. (1855): animal brunneo-olivaceum, unicolor; testa parte anteriore sat crassa, calcarea, opaca, sibrotunda, triplo majore quam testa externa; long. anim. $70-80 \mathrm{~mm}$. (Gallia ad Crau.) Animal clypeo antice minus libero, testa 11-12 $\mathrm{mm}$. longa, $7 \mathrm{~mm}$. lata, $2-2^{1 / 4} \mathrm{~mm}$. alta, interna facile liberanda. Fig. M. Tand. Hist. II. t. 4 f. 19, 20.

\section{Gen. Vitrina Draparnaud.}

Animal elongatum, gracile, sed magnum, pallio antire a testa producto, transverse rugosa, in dextro latere appendirem linguaformem retrorsum super testam emittcnte; orifrinm respirationis in parte dextra et postica murginis pallii; orificium genitale in cervicis latere dextro et mediano.

Testa imperforata, depresso-globosa vel aniformis, perTucida, tenuissima, hyalina, spira perbrevi; anfr. 2-3, releriter accrescentes, ultimus maxime dilatatus; apertura ampla, peristomate tenui, margine columellari membranacenmarginata.

Syn. Hetix MüLlen, 1774. - Vitrina Drap., 1801. Helicolimax FẺr. sen., 1802. - Vitrim MIONTF., 1810. - Hyalina Stud., 1820. - Limacina Harts., 1821. Testacella sp. ОкеN, 1815.

Habitat in muscis et inter folia putrescentia locis humidis, ubi per totam hyemem, tempestate leni et pluviosa, hujus generis species capias. Verno tempore ova ponunt, pulli per astatem in terra occulti forte crescunt et cum autumno in lucem maturi veniunt. Fossilis in str. eocenico infero, miocen. et pleistocen. medio et supero.

1. Phenacolimax StAB. Testa plus minus globulosa, anfr. 3-4, apertura plus minus rotundata, margine columell. angustissime membranaceo-marginata. Animal totum in testa continetur et epiphragmate vitreo temussimo clauditur.

\section{+ Testa lavis vel lavigata.}

1. V. pellucida MüLt. (1774): t. subgloboso-depressa, rotundata; anfr. celeriter accrescentes, ult. tumido, antice vix dilatato; spira convexa; sutura crenulata; long. $4-5$, lat. 3-4, alt. 3-4 mm. (Europa.) T. nitidissima, virescens, apertura obliqua, rotundato-ovalis, lalit. et long. subærualis. - Syn. Hel. limacoides v. Alt. 1812. V. beryllina C. Pfr. 1820. V. subglobasa Mich. 1831. Fig. Rssm. Icon. f. 28, Kob. Nass. F. t. 1 f. 7. (Foss.?) 
- valr. perforuta mh.: t. minor, convexior et rotundatior. perforata, margine columellari basi reflexiuseulo. (Suec., Gall. \& c.) Forma inter V. pell. et annul. sicut media.

2. V. major F́́r. (1807): t. depressa, ovato-rotundata; anfr. celerrime accrescentes, ult. antrorsum elongato, depresso. basi subplano; spira subplana; sutura filo-marginata; long. 5-8, lat. $4^{1 / 2}$ $-6 \frac{1}{2}$, alt. $3-4 \mathrm{~mm}$. (Gall., Helv., Tirolia.) T. nitida, virescens, striatula, apertura perobliqua, elongato-elliptica, latior quam alta. Syn. V. pellucida Drap., 1801. V. draparnaldi Cuv.; 1817. Fig. Nachr.bl. 1871. t. I f. 7.

- var. clliptica Brown (1819): t. minus depressa, anfr. ult. minus dilatato. (Germ. in Taumus et Siebengebirge, Belgin.) V. major et draparnaldi Ancet. germ.

$$
\text { ti T'este armule striatule } v \text { plicosa." }
$$

3. V. annularis VEx. ap. STUd. (1820): t. vix nitidula. depresso-globosa, orbicularis; anfr. lente accreseentes, ult. rotundato, antice non dilatato; spira prominula, obtusa; sutura sit profunda; long. 4-5, alt. $21 / 2-3 \mathrm{~mm}$. (In region. altior. A7pium \&e. Ital. bor., Ilelv., Tirol., Pyren., Lusit., Hisp., Gall., Transsilv., Sicilia; Belgia ad Chaulfontaine, teste clar. Broeck.) 'I'. sæepissime perforata, irregularitel' plicosa, viridnla, apertura parum obliqua, rotundata. Syn. V. musigneni Mandral. 1842. V. plicosa Bielz, 1867.

4. V. servainiana S:T Sıм. (1870): t. nitidissima, subglobosa; anfr. regulariter accrescentes, ult. leviter dilatato, subcompresso; spira convexa; sutura impressa; long. 5, alt. $3 \mathrm{~mm}$. (Gall. in Pyren. alt.) T. imperforata, argute striatula, pallide albidovirescens, apertura obliqua, rotundato-oblonga, marg. colum. superne supra locum umbilicalem expranso.

2. Semilimax STAB. Testa depresst, anfr. 2-3 rapide acerescentes, apertura plus minus horizontalis et auriformis, margine colum. membrana reflexa prodita. Animal in teste neutiquam contineri potest.

† Spira fere dimidium latitudinis testre efficiens.

5. V. diaphana DrP. (1805): to ublonga, auriforme dilatata, apertura ampliata, marg. colum. perarcuato, membrana marg. lata; long. 6-7, lat. 41/2-5, alt. 4-5 inm. (Gall., Helv., Bosna, Serbia, Ital. bor., Hisp. ad Madrid, Germ., exel. bor.) T. vitrea v. virescens, intus paullulum margaritacea, anfr. 2-3, forte accrescentes, spira depressa, apertura antice subtruncata. Syn. Helix limacina 
v. Alten, 1812. Hyalina vitrea Stud. 1820. Fig. Nachr. bl. 1871. t. 1 f. 3, 10. - V. heynemanni C. Koci (1871), longitudine membranæe colum. distincta, est forma V. diaph. minoris ponderis, teste am. Cless. (Exk. Fauna, 1876.) Species ita a clariss. Koch descripta: t. perdepressa, spira plana, anfr. $2 \frac{1}{2}$, penultimus diametro dimidium longitudinis totius non attingens, apert. elongata, margine anteriore arcu levi truncato, posteriore alte arcuatim exciso, limbo membranaceo marginis columellaris spiraliter continuati cincto, limbo nitidissimo, partem inferam anfr. ultimi æequante, ab hac linea distincta carinali separato; long. 6, lat. $4^{1 / 2}$, alt. $8 \mathrm{~mm}$. Fig. Nachr. bl. 1871. t. 1. f. 4,9 .

- var. glacialis Forbes (1837): apertura antice rotundata, membrana margin. colum. perlata, spira $1 / 2$ long. testæ non occupaus. (Alpes altior.)

6. V. charpentieri SтAв. (1859): t. elliptica, apertura rhomboidea, ovata, marg. colum. arcuato, membrana marg. angusta; long. 5-6, alt. $2 \frac{1}{2}-3 \mathrm{~mm}$. (Alpes Helv., Tirol., Pedemont.) Differt a præcedente præeterea apertura breviore et altiore, spira elatiore, anfr. 3 lentius crescentibus, ultimo longius producto et antice dilatato. Syn. $\boldsymbol{V}$. nivalis Charp. ap. Dum. \& Mort. 1852.

\section{tt Spira brevissina, $1 / 4$, ad summum $1 / 3$, latitudinis te- stce efficiens.}

7. V. pyrenaica FÉR. (1822): t. depressa, ovalis; anfr. $21 / 2$, ultimus membrana angustissima brunnea cinctus; apertura horizontalis, fere regulariter ovalis, intus callosa, marg. dextro medio dilatato, colum. leviter arcuato; long. 6 , lat. $3^{1 / 2}-4$, alt. $21 / 2$ mm. (Pyrence.) T. hyalino-virens, polita, apertura magna, $3 / 4$ long. testæ occupans, obliqua, subelliptica, antice rotundata, intus lactea, membrana marg. $1 / 4$ partis infer. testæ.

8. V. - brevis FÉR. (1822): t. depressa, elliptico-auriformis ; anfi: 2, rapide accrescentes; apertura subhorizontalis, elliptica, marg. dextro medio repando, colum. forte arcuato, marg. colum. membranaceo sat angusto; long. 5, lat. 4, alt. $2 \mathrm{~mm}$. (Germ.merid.-occ., Tirolia, Carinthia.) T. hyalina, nitidissima, subrotundata, spira non 1/4 long. testæ, apertura ampla, antice truncata, dilatata, membr. colum. vix $1 / 3$ partis infer. testæ. Fig. Nachr. bl. 1871.t. 1 f. 8.

9. V. elongata DRP. (1805): t. depressissima, elongato-auriformis; anfr. vix 2, celeriter accrescentes; apertura fere horizontalis, oblongo-ovalis, marg. dextro medio repando, colum. perarcuato, marg. membr. lato; long. 4-5, lat. 3, alt. 11/2-2. (Germ., Carinth., Gallia.) T. lutescenti-hyalina, supra visa subrhombea, nitida, spira 
fere $1 / 3$ et apertura $2 / 3$ long. testæ occupantes, apertura antice truncata. Fig. Nachr. bl. 1871. t. 1. f. 5.

\section{Gen. Hyalinia (Férussac) Agassiz.}

Animal pallio crasso, pede angusto; orificium respirationis in latere dextro supero colli, genitalis ad basin colli; sagitta amatoria ejusque bursa et folliculi mucosi omnino desunt; porus mucosus, in apice pedis, mullus. Epiphragma mulla rel hyalina.

Testa sapius umbilicata, interdum perforata, depressa, rarissime orbiculato-convexa, polita, tenuis; anfr. 4-7, regulariter accrescentes $v$. angusti; apertura rotundata $v . l u-$ naris.

Syn. Helix Aucct. prisc. - Hyalines FÉR., 1821. Hyalinia Agass., 1837. - Hyalina Gray, 1842.

Habitat in silvis, locis umbrosis, in plantis, foliis putrescentibus, in muscosis humidis juxta aquas, in pratis, in rimis scopulorum murorumque, imo in cellis. Fossilis in strat. omuibus a form. cretaceo supero.

1. Conulus Fitz. Testa turbinato-globosa, subimperforata, anfr. 5-6, tardissime accrescentes, apertura depressolunavis. Uncini radule marginales apiculo secundario.

1. H. fulva MüLL. (1774): t. fulva, striatula, nitida; apertura latior quam alta; diam. $21 / 2-31 / 2$, alt. $2-3 \mathrm{~mm}$. (Europa.) Syn. Helix fulva MüLt. (Foss.)

- var. alderi Gray (1840): t. minor, obscure brunnea, lævigata, polita.

- var. mortoni JEFFr. (1830): t. obscura, depressa, utrinque fere æqualiter convexa.

2. Hyalinia Agass. Testa depressa, umbilicata, anfr, $5-7$, regulariter accrescentes, apertura rotundato-lunaris. Uncini vadule marginales hamiformes, non denticulati.

+ Cellaric: majores (diam. 10-15-20 mm., rarissime $7-9 \mathrm{~mm})$; testa subtus albida.

*) Anfir. lente accrescentes (spira circulum magnum v. maximum formans).

a) Anfractus ultimus latior (antice tamen non dilatatus).

a) Testa umbiticata, firma, nitida, vix striatula, depressa, subtus albida, sutura plus minus canaliculata. 
2. H. cellaria MÜLL. (1774): t. aperte umbilicata, nitida, irregulariter striatula. virenti-cornea, spira depressa, sutura impressa, linea obscura marginata; diam. maj. 12, min. 11, alt. 4 mm. (Europa.) T. anfr. 6, planulatis, lente accrescentibus, ult. depresso; apertura obliqua, ovali-lunaris, marg. columellari non reflexo. Fig. Rossm. Icon. f. 527. (Foss.) Variat in Eur. merid. major: diam. 15, alt. $6 \mathrm{~mm}$, in Sicilia maxima: diam. maj. $16 \mathrm{~mm} .13$, alt. $8 \mathrm{~mm}$.

- var silvatica Mörci (1864): t. sat convexa, apertura intus precipue in margine columellari late albo-sublabiata; diam. 11-13, alt. 5-6 mm. (Dania, Suecia, Britan., Belgia.) Syn. Zon. draparnaldi Bk? Westerl. 1873. Hyal. cell. v. elevata Broeck 1869.

- var. margaritacea Scm. (1851): t. margaritaceo-diaphana, concolor, anfr. $4 \frac{1}{2}$, teretes, ult. nec dilatatus, nec deflexus, apertura lunato-rotundata; diam. maj. 101/2, min. 9, alt. $5 \frac{1}{2} \mathrm{~mm}$. (Germ. in hortis Ascania, Dan.) Forma albina: t. albida, coerulescente, subopaca.

3. H. villæ MorT. (1853): t. umbilicata, nitida, vix striatula, cornea; spira planiuscula, sutura canaliculata, non marginata; diam. maj. 14. min. 12, alt. $5 \mathrm{~mm}$. (Lombardia, Carinthia ad Bad Villach; Sicilia in ins. Favignana: Rossm.) T. subdiscoidea anfr. 6, planiusculi, lente accrescentes, extus lente declivis, ad suturam obtuse angulati, intus breviter descendentes, quasi incumbentes, ult. ntrinque depressus, latus; apertura vix obliqua, lunaris. Syn. Zon. eugyrus Stab. 1859; Hel. mortilleti Stab. ap. Pfr. 1859. Fig. Mal. Bl. 1856, t. 2 f. $1-3$.

4. H. de natale Ben. ap. Prr. (1856): t. late umbilicata, subopaca, minutim striata, subglabra, fusco-cornea; spira plana $\mathrm{V}$. medio concava; sutura subcanaliculata; diam. maj. 12, min. 111/2, alt. $5 \mathrm{~mm}$. (Sicilia.) T. discoidea, raro corneo-luteola, latitudine infra medium anfr. ultimi maxima (ap. H. villæ supra medium), anfr. 6-7 angusti, lentissime accrescentes, ult. major, subcarinatus, apertura obliqua, triangulari-lunaris, marg. basali areuato-sinuato. Fig. Rossm. Icon. f. 902.

\section{(H. pictonica Bourg.)}

b) Testa perforata vel peranguste umbilicata, tenuis, petlucida, nitidissima, sublavis, convexo-depressa, circa umbiticum pallidior; suturu superficialis.

5. H. glabra STUD. ap. FÉR. (1822): t. perforata, convexodepressa, tenuissime striata, supra fulva; apert. elliptico-lunata; diam. 11, alt. $6 \mathrm{~mm}$. (Europa media.) T. pertenuis, fragilis, diaphana, anfr. 5-6, vix convexiusculi, ult. paullulum depressus, spira 
convexa, apertura obliqua, parum depressa, subelliptico-lunata, marg. longe distantibus, colum. extense arcuato. Fig. Rossm. Icon. f. 528. (Foss.)

- var. nitidissima PARr. ap. Mouss. (1859): t. summo depressiusculo, anfractibus subrotundatis, subtus convexioribus. (Epirus.)

- var. subglabra Bourg. (1860): t. argutissime striatula, præsertim ad suturam, sutura impressa, apertura lunato-oblonga; diam. 13-16, alt. 6-9 mm. (Gall. ad Vannes.)

6. H. ercica Ben. ap. PFr. (1868): t. perforata, convexodepressa, vitracea, corneo-virens, concolor; apertura depressa, subovata, oblique lunaris; diam. maj. 10, min. 8, alt. $5 \mathrm{~mm}$. (Sicil. in mont. Caron.) T. diaphana, nitidissima, anfr.5, vix convexiusculi. Fig. Ben. Ill. Sic. t. 10 f. 5. Helix glaberrima Ben. 1859, non Pfr. 1854. An forma vitracea $H$. glabræ?

7. H. alliaria MiLL. (1822): t. subaperte umbilicata, depressa, sublavis, fulvo-comea; apert. rotundato-lunaris; diam. 6-6, alt. 3-4 mm. (Scand., Britan., Islandia, Ferö, German. bor., Gall. bor.) T. tenuis, pellucida, anfr. $5-5^{1} / 2$, vix convexiusculi, ult. rotuntato, spira convexiuscula, apert. parum obliqua, marg. columell. forte arcuato. Variat viridula, testa vitrea. Animal nigrum, foetidum (allio oleraceum).

- var. anceps Westerl. (1871): t anguste umbilicata, depressior, viridula, apertura diagonalis, lunato-ovalis, margine colum. levissime arcuato. (Suce. ad Lund.)

- var. aquitanica CH.: t. magnitudine et convexitate $H$. glabræ, umbilico latiori. H. alliariæ similis. (Ital. Toscana.)

8. H. natolica ALB. (1857): t. anguste umbilicata, convexodepressa, levissime striatula, sub lente lineis spiralibus subtilissimis, fusco-cornea; apertura ovato-lunaris, marginibus subconniventibus; diam. maj. 20, min. 17, alt. $8 \mathrm{~mm}$. (Transsitv.) T. ad suturam crispata, anfr. 6-7, convexi, apert. margine basali strictiusculo, declivi. Fig. Mal. Bl. IV. t. 1 f. 4-6.

c) Testa umbilicata, nitidula, superne, confertim striata, subdiscoidea, sutura impressa.

9. H. opaca Schutrl. ap. Alв.: t. subdiscoidea, confertim striata, parum nitens; anfr. ult. depressus, subangulatus; apert. ovali-lunaris; diam. maj. 9, min. 8, alt. $4 \mathrm{~mm}$. (Sardinia.) T. mediocriter umbilicata, cornea, spira vix elevata, sutura profunda, anfr. 5 , convexiusculi, apert. depressa, marg. colum. declivi, subarcuato. syn. Helix balmei Pfr. Mon. III. p. 85. Fig. Küst. Mon. 150, 6-8. 
10. H. superflua Rssir. ap. PFR. (1848): t. depressa, striata et lineis spiralibus minutissimis decussata, nitidula; anfr. ult. teres; apertura lunato-circularis; diam. maj. 18 , min. 15, alt. $8 \mathrm{~mm}$. (Creta.) T. umbilicata, solidula, diaphana, cinnamomea, spira planiuscula, anfr. 5-6, convexiusculi, apert. parum obliqua, marginibus conniventibus, Fig. Küst. Mon. 121. f. 10-12.

11. H. alicuvensis BEN. (1857): t. subdepressa, oblique striata; anfr. ult. basi planulatus; apertura lunaris; diam. maj. 9, min. 8 , alt. $4 \mathrm{~mm}$. (Ins. Alicuri pr. Sicil.) T. mediocriter umbilicata, tenuis, subdiaphana, spira convexa, anfr. 5, apert. marg. colum. leviter sinuato. Fig. Ben. Ill. Sicil. t. 3 f. 15.

12. H. planella Prr. (1853): t. subdiscoidea, rugulosa-striata, oleoso-micans; anfr. ult. perdepressus, subangulatus; apert. diagonalis, depresse rotundato-lunaris; diam. maj. 10 min. 8 , alt c. 4 mm. (Sicilia.) T. sat late umbilicata, cornea, tenuissima, pcllucida, spira subplana, anfr. 5, vix convexiusculi, apert. marginibus vix convergentibus, colum. levissime arcuato.

?. H. peregrina ARAD. \& MAGG. (1839): "t. subdepressa, oblique ac. eleganter striata, absque nitore; anfr. 5-6 rotundati; sutura profunda; apertura oblique subovata; diam. $31 / 2$ lin., alt. 2 lin." (Sicilia.) Species dubia, teste cl. Benoit.

ß) Anfractus uitimus vix major. (Testa arctispira, orbiculata.)

13. H. testæ PHIL. (1844): t. anguste umbilicata, convexodepressa, arctispira, subtiliter striatula; anfr, ult. demum breviter decurvatulus; sutura linearis;diam. 121/2, alt. $7 \mathrm{~mm}$. (Sicil.) T. corneo-lutescens, nitida, subtus albida, planiuscula, spira convexiuscula, anfr. 7, lentissime accrescentes, planulati, apertura obliqua, lunata. Syn. Hel. philippii Testa. Fig. Rossm. Icon. f. 903. Cfr. H.de natale.

- var, rossmassleri mh.: t. minor, latius umbilicata, anfr. 6 , minus lente accrescentes; diam. 10, alt. $4^{1} / 2 \mathrm{~mm}$. (Sicilia.) Fig. Rossm. Icon, f. 904.

14. H. frondosula Mouss. (1863): t. mediocriter umbilicata, convexo-depressa, arctispirata, frondoso-striata; anfr. ult. non decurvatus; sutura perimpressa; diam. maj. 9 , min. 8 , alt. $31 / 2 \mathrm{~mm}$. (Constantinop.) T. fusco-cornea, nitidula, subtus pallidior, spira convexa, anfr. 7 , densi, convexi, ad suturam tumiduli, apert., vix obliqua transverse depresso-lunaris.

15. H. diaphanella KRYN. (1837): t. anguste umbilicata; depressa, sublævis; anfr. ult. non decurvatus; sutura impressiuscula; 
diam. 9, alt. $21 / 2-3 \mathrm{~mm}$. (Tauria.) T. diaphana, griseo-albida aut flavescens, subtus plamulata, anfr. 7 planulati, ult. attenuatus, apertura compresso-lunata. Syn. H. multispira Pfr. Symb.

16. H. pazi BourG. (1866): t. umbilicata, subdiscoidea, argute ac oblique striatula; anfr. lente accrescentes, ult. ad aperturam subito descendens; sutura sat impressa; diam. 11, alt. $5 \mathrm{~mm}$. (Hisp. ad Madrid.) T. tenuis, cornea, anfr. 7, convexiusculi, ult. sicut incumbens, supra convexus, sultus leviter compressus, apert. perobliqua, valde lunata, subtriangulari-oblonga. Fig. Bourg. Moll. nouv. \&c. VII. t. 33 f. 1-6.

17. H. pictonicus Bourg. (1870): t. umbilicata, compressoplanorbiformis, lrvigata ac circa suturam eleganter radiatulostriatula; anfr. lente accrescentes, ult. non descendens; sutura impressa; diam. $12^{1} / 2$, alt. $5 \mathrm{~mm}$. (Galliu.) T. cornea; anfr. 6 convexiusculi, ult. subcompresso-rotundatus, subtus prope aperturam compressior, apertura leviter obliqua, sat lunata, transverse compressorotundatn, marginibus remotis. Fig. Bourg. Rev. Zool. 1870. t. 16 f. $7-9$.

H. uzielli Iss. (1872): Pisa. Species mihi ignota.

**) Anfr. celeviter accrescentes (spira circulum mediocrem v. parum formans)

18, H. lucida Drap. (1801): t. convexa, corneo-fusca, subtus lactescens, ad umbilicum mediocrem declivus; anfr. ult. multo major, tectiforme inclinatus; apert. perobliqua, depressa, oblonga; diam. 14-16, alt. 6 mm. (Gall., Brit., Germ., Ital., Corsica.) T. sape virenti-cornea, irregulariter striatula, sutura impressa, anfr. 5-6, vix convexiusculi, ult. subdepressus, apert. lunari-subovalis, marg. colum. subincrassato. Syn. Hel. nitida Drp 1805; H.draparnaldii Beck 1837. Fig. Dup. Hist. t. XI. f. 1. Variat 1) major: d. 18 a. $10 \mathrm{~mm}$. ; 2)' albina.

- var. blanneri Schutrl. (1843): t. nitidior, compressior, planior, apert. magis oblonga. (Gall. merid., Corsica.)

19. H. farinesiana Bourg. (1870): t. supra convexo-tectiformis, cornea, subtus pallidior, sicut concava, ad umbilicum infundibuliforme decliva; anfr. ult. paullo major; apert. lunata, oblique declivis et oblonga; diam. 15, alt. $7 \mathrm{~mm}$. (Gall., Hisp.) T. sublævigata, lata umbilicata, anfr. 6-61/2, convexiusculi, sutura parum impressa. Fig. Rev. Zool. XXII. t. 16 f. 1-3.

20. H. obscurata Porro ap. Villa (1841): t. discoidea, fuscocornea, subtus vix pallidior, umbilico mediocri; anfr. ult. maximus; apert. obliqua, late lunaris; diam. maj. 20, min. 17, alt. 7 
mm. (Italia bor., Sicil., Corsica, Sardinia.) T. valde depressa, subplanorbiformis, lavigata, tenuis, anfr. $5 \frac{1}{2}$, convexiusculi, ult. velociter dilatatus, latus, depressus, spira plana, sutura impressa. Fig. Chemn. Conch. Cab., Helix t. 121 f. 16-18.

- var. septemtrionalis Bourg. $(1870)$ : t. cornea, apert. minus obliqua, transversali-oblonga, lunata, marg. dextro antrorsum arcuato ac sat provecto; d. 15, a. $5 \mathrm{~mm}$. (Gall. septemtr.). Fig. Rev. Zool. XXII. t. 16 f. $4-6$.

21. H. navarrica Bourg. 1870): t. supra subtusque convexiuscula, cornea, subtus vix pallidior, anguste-umbilicata; anfr. ult. paullo major; apert. parum obliqua, oblongo-rotundata, lunata; diam. 12, alt. 51/2 mm. (Hisp., Gallia.) T. sublievigata, anfr. 6 subconvexiusculi, ult. subtus convexior. Fig. Rev. Zool. XXIl. t. 16 f. $10-12$.

22. H. æquata Mouss. (1854): t. valde depressa, corneoflavida, sat late umbilicata; anfr. ult. latus; apertura diagonalis, transverse lunato-ovalis; diam. 15, min. 11, alt. $6 \mathrm{~mm}$. (Rhodus, Syra, circa Athenas.) T. tenuis, leviter striatula, sub lente obsoletissime spiraliter striata, parum nitens, anfr. $4^{\mathbf{1}} / 2$, rapide accrescentes, subplani, ult. depressus, basi convexior. Fig. Mouss. Coq. Bell.t. 1 f. 1.

23. H. deila BourG. (1857): "t. depressa, argutissime striatula, fulvo-olivacea, fragilis, diaphana, umbilicato-perforata; anfr. 6, ult. major; apert. obliqua, semirotundata, magna; diam. 11, alt. 6 mm." (Sebastopolis.) Fig. Rev. Zool. 1857. t. 8 f. 7-9.

24. H. nitens Mich. (1831): t. depressa, lutescens, virescens v. fuscescens, oleo micans, aperte umbilicata; anfr. ult. depressus, antice valde et subito dilatatus; apert. ovato-elliptica; diam. 9-11, alt. $4 \mathrm{~mm}$. (Europa merid. \& occid., forma minor septemtrionem versus usque ad Daniam et Germ. sup.) T. subovata, tenera, diaphana, glabriuscula, subtus albida, anfr. $4^{1} / 2$, planulati, ultimus multo major, |depressus et antice subdeflexus, sutura parum impressa, spira non circularis, apert. subovalis, parum lunata. - Fig. Rossm. Icon. f. 524, 525. (Foss.?) Variat 1) minor: d. 7-9 a. 3-31/2 mm., 2) helmi GilB.: t. lutescens, unicolor (Dan. Helv. Brit. Gall. mer.).

- var hiulca JAN. ap. ALв. (1850): t. major, paullo convexior, apert. major, rotundior. (Carinth., Ital. sup., Gill.) Fig. M. Tand. Hist. t. XIX. f. 17.

- var. sicula: t. solidior, subtiliter striata, subopaca, anfr. ult. minus dilatatus. (Sicilia.)

- var. dutaillyanus MAB. (1868): t. minor, sutura impressa, apert. vix obliqua, anguste oblonga. (Gall.merid.-orient., Helv.\&c.) 
25. H. nitidula Drr. (1805): t. convexo- depressa, rufescens v. rufo-brunnea, circa umbilicum lactescens, aperte umbilicata, nitida; anfr. ult. tumido-convexus, antice minime dilatatus; apert. rotnndato-lunaris; diam. 7-9, alt. 31/2-41/2 mm. (Europa media et occid.: a Suec. et Norv. media ad Transsilv., Ital. bor. et Hisp. austr.) T. rotunda, firma et solida, sublævis, anfr. $4-4^{1} / 2$, convexiusculi, sutura impressa. Fig. Rossm. Icon. f. 526. (Foss.) Species me judice a preced. bene distincta, caveas tamen ne formas minores illius pro hac capias.

- var. lucens Pult.: t. brunnea, concolor, sine albidine basali. (Penins. Cimbr. et Britannia.)

if Nitidosa: minores (diam. ad summum 4-7 - et N:o 33,9 - mm.), testa scepius regulariter striata vel striatula, concolor.

*) Testa minutissima (lat. et alt. $1 \mathrm{~mm}$.), subglobosa, anbuste umbilicata.

26. H. lawleyana Bourg. (1863): t. albido-crystallina, sublævigata; spira convexo-tectiformis, obtusissima; apertura parum obliqua, lunato-rotundata. (Italia, in alluviis fluminis Arno prope Fiarenza lecta.) T. hyalina, fragillima, anfr. $4^{1 / 1} / 3-5$, convexiusculi, regulariter crescentes, sutura impressa separati, ultimus paullulum major, in medio subangulatus. Fig. Bourg. Moll. nouv. \&c. III. t. 8. f. 11-14. "Persimilis H. petronella Ch., sed minutissima."

**) Testa parva $\left(3^{1} / 3-5 \mathrm{~mm}\right.$.), depressa, anfr. regulariter accrescentes.

a) Sutura levis, marginata et crenulata, anfr. ad suturam plani; testa striata.

27. H. circunlineata KüsT. ap. PFR. (1846): t. subdiscoidea, superne lineis confertis spiralibus sculpta; anfr. 5, angusti; apert. verticalis, late lunaris; umbilicus latus; diam. $51 / 2$, alt. 2 mm. (Dalmatia ad Budua.) T. tenera, hyalina, suborbiculata, sutura sat impressa, anfr. vix convexiusculi. Fig. Chemn. Cab., Helix, t. 24 f. $22-25$.

28. H. hammonis Хтвӧм (1765): t. spira depressa, subplanata, dense striatula; anfr. 4, planati, ultimus depressus, dilatatus; apert. lunato-ovalis; umbilicus angustus; diam. $3 \frac{1}{2}-4^{1 / 2}$, alt. $2 \mathrm{~mm}$. (Europa.) T. cornea, animale obscure cinereo foeta castanea, apert. sæepe albo-labiata, deflexa. Syn. Hel. striatula Gray, 1821 (nomen); H. nitidosa Fér., 1822 (nomen); H. radiatula Alder 1830. Fig. Rossm. f. 23. (Foss.) - Variat viridula Menke (1820) pr. p: t. albidovirescens, hyalina. 
29. H. petronella Charp. ap. Pfr. (1853): t. spira late convexa, confertim costulato-striata; anfr. $4 \frac{1}{2}-5$, planulati, ultimus rotundatus, antice minime dilatatus; apert. lunato-subrotunda; diam. $4 \frac{1}{2}-51 / 2$, alt. $2 \frac{1}{2}-3 \mathrm{~mm}$. (Eur. bor., media in region. montosis et in Alpibus.) T. virescens, hyalina, animale cinereo foeta cinerea, anfr. ult. subtus sat tumidus, rotundatus. Forma præcedentis viridula sæpe pro hac perperam sumitur. Syn. H. viridula Auctt. plurim. Fig. Mal. B1. 1858. t. 1 f. 1, 2, - Zon. jaccetanicus c Barcelona in Hispania, a clar. Bourguignat 1870 descripta et in Rev. Zool. e. a.t. 16 f. 23-28 delineata, certe forma cornea hujus speciei.

ß) Sutura impressa, immarginata; anfr. ad suturam scpius tumiduli; testa levigata.

30. H. pura ALD. (1830): t. plus minus effuse umbilicata, confertim et subtilissime striata; anfr. 4, regulariter accrescentes, ult. paulo major, depressus; diam. $4-4^{1} / 2$, allt $2 \mathrm{~mm}$. (Europa, præcipue montana.) T. vix nitidula, cinereo-albo, parum pellucida, animale albido antice fusco foeta anfr. 3 primis fulvis; apert. lunulatorotundata. - Hel lenticularis Held (1837) est forma cornea hujus speciei. Hæc in Eur. boreali, illa (pura) in Eur. merid. perrara.

31. H. clara HELD (1837): perforato-umbilicata, lævissima; anfr. $4 \frac{1}{2}$, celeriter accrescentes, ult. multo major, dilatatus; diam. $4 \frac{1}{2}$, alt. vix $2 \mathrm{~mm}$.) (Bavaria in Alp., Helvetia in monte Pilatus.) T. nitida, hyalina, lactescenti-albidula, supra plana, anfr. infra rotundata, spira circulum majorem qvam in præc. formans. Syn. Zon. pilaticus Bourg. 1862 in Malac. d. quatr. cant. t. 1 f. $6-10$.

32. H. courquini Bourg. 1870): t. profunde pervio-umbilicata, lævigata; anfr. 5, regulariter crescentes, ult. vix major, compresso-rotundatus; diam. 6, alt. $2 \frac{1}{2} \mathrm{~mm}$. (Hisp., Sarria prope Barcelona.) T. supra vix convexa, nitida, subpellucida, pallide cornea, apert. obliqua, lunata, transverse oblonga, marg. supero antrorsum arcuato ac provecto. Fig. Rev. Zool. XXII. t. 16 f. 17-22.

33. H. rigiaca BounG. (1862): t. perforata, levis; anfr. 6, regulariter accrescentes, ultimus magnus, dilatatus, infr. subplanatus; diam. 9, alt. $3 \mathrm{~mm}$. (Helvetia in monte Rigi.) T. albidocrystallina, nitida, anfr. planulati, apertura vix obliqua, lunato-oblonga. Fig. Rev. Zool. 1862, t. 18 f. 1-5.

y) Testa major (6-7 mm.) circa umbilicum excavata; anfr. lente accrescentes, convexi; sutura profunda.

34. H. simplicula Mor. (1845): t. depressa, striatula, vix nitidula; anfr. ultim. superne subangulatus; apert. subverticalis, depressa, lunaris; diam. $6 \frac{2}{3}$, alt. vix $3 \mathrm{~mm}$. (Lusitania, inter 
Iertala et Castro Verde.) T. diaphana, pervie umbilicata, pallide cornea, apert. marg. basali levissime arcuato. Fig. Chemn. Cab. Helix, t. 128. f. $31-33$.

35. H. excavata BEan ap. Ald. (1830) t. depressa, dense striata, nitida; anfr. ult. angustus, subcylindraceus; apertura rotundo-lunata; diam. 61/4, a. $2^{3 / 4} \mathrm{~mm}$. (Britannia; Penins. Cimbr. ad Flensborg?) T. late et profunde umbilicata, pellucida, cornea, apert. obliqua, subcircularis, perprofunda. Variat viridula hyalina.

36. H. nitida MüLL. (1774): t. globoso- depressa, striata, nitida; anfr. ult. latior, teres; apert. rotundato-lunaris; diam. 6, alt. $3 \mathrm{~mm}$. (Europa.) T. aperte umbilicata, subcastaneo-brunnea v. rufescenti-cornea, animale foeta subnigra, nitidissima, anfr. 5, regulariter sed multo fortius quam in præcidentibus accrescentes. Pro hac specie beat. Leniman genus Zonitoides 1862 creavit: animal sagitta amatoria, ejusque bursa et folliculis mucosis præditum. Syn. Hel. lucida Drap. 1805. Fig. Rossm. Icon. f. 25. (Foss.) Variat viridula et albida; variat anguste umbilicata et striatula.

3. Vitrea FIтz. Testa scepius perdepressa, orbicularis, vitrea, anguste umbilicata, perforata, diaphana, nitidissima; anfr. 4-6, angusti; apertura verticalis, lunaris.

*) Testa imperforuta (vel subrimata).

37 H. diaphana STUD. (1820): t. anfr. lente crescentibus, apertura anguste lunata; diam. $4-4^{2} / 3$, alt. $1 \frac{1}{2}-2 \mathrm{~mm}$. (Germ., Gall., Alpes, Transsilv., Dalm., Ital. bor., Sicil., Corsica, Hisp.) T. subnivea, supra subplana, anfr. 5-6, planulati. Syn. Hel.hyalina Fér., 182\%. Hyal. vitrea Bielz, 1867. Fig. Rossm. Icon. f. 530. M. Tand. Hist. t. 9 f. 30-32. (Foss.) - H. subrimata Reinh. 1871 est teste amic. Clessin (Exk. Moll. Fauna 1876, p. 81) nihil aliud ac H. diaphana juvenis et immatura, testa perforato-rimata. (Testa celeb. Reih. H. subrim. differt a H. diaphana: testa minore (diam. $2^{1 / 2}-3 \mathrm{~mm}$.), supra subtusque convexiore, anfr. $4^{1 / 2}-5$, fortius accrescentibus, colore viridulo; habitat in Moravia, Gallizia, Bukowina, Helv., Ital.)

38. H. erjaveci Brus. 1870): t. anfr. lentissime accrescentibus, apertura angustissime lunari; diam. $3^{2} / 3$, alt. $1 \frac{2}{3}$ mim. (Croatia.)

\section{**) Testa perforata vel anguste umbilicata.}

39. H. crystallina MüLL. (1774): t. perforata; anfr. $4^{1} / 2-5$, convexiusculi, lente accrescentes, ultimus major, rotundatus, subtus tunidus; apert. late lunaris, margine basali arcuato; diam. $3-4$, alt. $11 / 2$ mm. (Europa.) Apert. marg. basali usque ad insertionem subæqualiter arcuato. Fig. Bourg. Ameu. Mal. I.t. XX. f. 19-24. (Foss.) 
- var. subterranea BourG. (1856): t. minor, apertura albolabiata.

40. H. contracta WesterL. (1873): t. sat aperte unbilicata; anfr. 5-6, planulati, lentissime accrescentes, ultimus angustus, penultimo subæqualis, utrinque depressus; apert. anguste lunata, margine basali leviter curvato; diam. $2 \frac{1}{2}$, alt. $1 \mathrm{~mm}$. (Suecia, German. septemtr., Gall. mevid.) Apert. margine columellari brevi verticali cum margine basali substricto angulum, rectum formante. Figurae hujus speciei in Jahrb. d. mal. Ges. 1875. t. 2 et Exk. Moll. Fauna p. 79 ab amiciss. Clessin datæ nullimodo testæ meæ congruunt. Forma testæ a latere visæ et præcipue aperturæ prorsus alia. - H. botteri Parr., Pfr. Mon. III. p. 66, ("differt a H. cryst. umbilico aperto et diam. $2 \frac{1}{3} \mathrm{~mm} . "$ ), in Dalmatia, Græcia et Italia boreali inventa, ab H. contracta, ut videtur, non diversa.

41. H. kutchigi PARr. ap. Pfr. (1865): t. umbilicata, anfr. 6, convexiusculi. arcte convoluti; apert. parum obliqua, anguste lunaris; diam. 5, alt. $2 \mathrm{~mm}$. (Dalm. ins. Lacroma.) T. levissime striatula, sutura impressa, anfr. ult. subtus paullo convexior, apert. margine supero brevissimo, basali usque ad insertionem subæqvaliter arcuato. "Superne persimilis H. hyalinæ Fér., sed sublate umbilicata."

42. H. hydatina Rossu. (1838): t. perforata; anfr. 5, planulati, ultimus major; apertura perobliqua, depressa, lunaris; diam. $6-6 \frac{1}{4}$, alt. $3 \mathrm{~mm}$. (Gracia, Italia.) T. albida, pellucida, striatula, sutura profundiuscula. Fig. Rossm. Icon. f. 529; Bourg. Amén. I. t. 50 f. $4-6$.

43. H. pseudohydatina BourG. (1856): differt a Z. hydatino statura minore, anfractibus minus globulosis, sutura non marginata, - apertura minus obliqua, angustiore, \&c. (Italia, Lusitania, Gallia.) "Differt a Z. cryst., cui affinior, statuŕa majore, apertura magis obliqua et oblonga, anfractibus rapidius accrescentibus, \&c." (B.). Syn. H. hydatina Aucct. plur. ital. \& gall. Fig. Dupuy Hist. t. XI. f. 5 .

44. H. latebricola Bourg. (1856): t. anguste umbilicata; anfr. 5, regulariter accrescentes, ultimus perdistincte major, obsolete carinatus; apert. obliqua, oblongo-lunaris; diam. 4-5, alt. $2^{1 / 4} \mathrm{~mm}$. (Gi'ceia) T. sublævis, albidissima, anfr. ult. supra subplanulatus, infra convexus. Fig. Bourg. Amén. I. t. 20 f. 7-12.

45. H. eudedalæa BourG. (1856): t. angustissime perforata; anfr. 6, regulariter accrescentes; apert. obliqua lunaris; diam. 7, alt. $4 \mathrm{~mm}$. (Grecia.) T. subcyaneo-albida, lævigata, anfr. convexiusculi, ult. infra subplanulatus, apert. margine columellari dilatato ac in perforatione reflexo. Fig. Bourg. Amén. I. t. 20 f. 1-3. 
4. Mesomphix Rafin. Testa magna, umbiticata vel raro subperforata, globoso-depressa, striatula, fusco-cornea, subtus pallidior; anfr. 51/2-7; apertura lunari-ovata, perist. simplici, acuto, recto.

*) Testa decussato-plicatula.

46. $\mathrm{H}$. fuscosa Z. ap. Rossy. (1838): t. anguste umbilicata, subdepressa, corneo- lutescens; apert. rotundato-lunata; diam. maj. 17, $\min .15$, alt $91 / 2 \mathrm{~mm}$. (Sicilia.) T. densissime et subtilissime transversim striata, lineis tenuissimis distantibus spiralibus decussata, subtus lævis, nitidissima, pallida; anfr. 51/3, angusti, ult. magnus. Syn. H. Calcare Arad. \& Magg. Fig. Rossm. Icon. f. 523.

\section{**) T. non decussato-linecita.}

47. H. olivetorum Hersr. 1784 (GMes. 1788): t. late umbilicata, depresso-globosa, lævigata v. ad suturam striata, olivaceocornea, basi vix pallidior; apert. ovato-lunaris; diam. maj. 31, min. 25, alt. $14 . \mathrm{mm}$. (Italia bor.; Bavaria ad Schellenberg.) T. parum nitens; anfr. 51/2, celeriter accrescentes, convexiusculi, ultimus magnus, antice dilatatus; apert. marginibus conniventibus. Syn. Hel. leopoldiana Charp. Hscr. Zon. mortilleti Pecch. 1868 - paullo postea $Z$. gerfalchensis nominata - est H. olivet. juvenis. Fig. Rossm. t. 39 (fig. sup. in medio.)

48. H. icterica Тıв.: t. late umbilicata, convexo-depressa, conferte arcuato-striata, straminea, supra fuscula; apert. lunatoovalis, intus margaritacea; diam. 27, min. 22, alt. $14 \mathrm{~mm}$. (Sicilia.) T. parum nitens, anfr. 51/2, celeriter accescentes, convexiusculi, ultimus magnus, antice dilatatus, subtus virenti-pallidus, apert.marginibus subapproximatis. Syn. Hel. calcarce Pfr., 1859.

49. H. incerta DRP. (1805): t. aperte umbilicata, globosa v: globoso-depressa, glabriuscula. supra corneo-rufa, subtus virenti-albida; apert. lunato-rotundata; diam. maj. 21, min. 17, alt. $12 \mathrm{~mm}$. (Gall. merid.) T. solida, nitida, anfr. $5 \frac{1}{2}-6^{1 / 3}$, convexiusculi, ultimo multo majore, rotundato, non antice dilatato. Syn. Hel. olivetorum Fér., Rossm., Dup. Fig. Rossm. Icon. f. 532.

50. H. malinowski ZeL. ap. Pfr. (1865): t. mediocriter umbilicata, convexo-depressa, sub lente subundulato-striatula, fulva, subtus pallidior: apert. Iunato-ovalis; diam. (sp. max.) $20 \%$, min. $17^{1 / 2}$, alt. $9 \mathrm{~mm}$. (Dobrudscha.) T. tenuiuscula, anfr. 6, convexiusculi, sat lente accrescentes, ultimus subdepressus, apert. intus leviter margaritacea, marginibus convergentibus.

51. H. filicum Kryn. (1827): t. anguste umbilicata, depressa, dense leviter striata, corneo-fusca, subtus virenti-albida; apert. 
rotundato-lunaris; diam. maj. 14, min. 12, alt. $6 \mathrm{~mm}$. (Tauria; Caucasus). T. nitidula, anfr. $6 \frac{1}{2}-7$, arcte voluti, planulati, ultimus antice dilatatus, apert. marginibus distantibus.

Gen. Zonites MontF.

Animal magnum, longum, pede distincte marginato; porus mucosus oblongus in apice pedis. Epiphragma membranacea. De cetero ut Hyalinia.

Testa magna, late umbilicata, orbiculato-convexa vel depressa, solidula, transversim striata et lineis spiralibus decussata, subtus nitida; anfr.6-7, sensim accrescentes, ultimus magnus; apert. obliqua, lunaris, intus leviter labiata.

Syn. Helix Auct antiqu,; Zonites Montfort, 1800. Helix Gr. Tragomma Heud, 1837.

Habitat hoc genus in silvis, sub foliis putridis, in muscis vel sub lapidibus. Fossilis in str. pleistocen. supero et medio.

+ Testa superne regulariter transversim striata et striis spiralibus argutis ornata (: regulariter granulata).

*) Testa carinata, sutura usque ad aperturam acute marginata.

1. Z. acies Partsch, Fér. (1821): t. convexo-depressa, superne rufo-cornea et a vertice densissime decussata; anfr. $61 / 2$; diam. maj. 31, min. 29, alt. 12 mm. (Dalm., Serbia, Bosna.) T. late umbilicata, supra convexa, subopaca, dense costato-striato, subtilissime decussata, subtus albida, lutescens vel coerulescens, infra carinam albidam dilute cornea; apertura lunato-securiformis. Syn. $H$. acutimargo Z., Brumati, Menke. Fig. Rossm. Icon. f. 152. (Foss.)

2. Z. crypta PArr. ap. PFr. (1868): t. lenticularis, spira vix elata, cinereo-carnea, anfr. penultimus supra suturam et ultimus omnino superne lineis spiralibus decussati; anfr. 5; diam. maj. 26, min. 23, alt $10 \mathrm{~mm}$. (Dalm., in carernis.) T. late umbilicata, subtus leviter striatus, nitidus, apert. obliqua, angulato-lunaris, intus pluries sublabiatum.

**) Testa obsolete carinata $v$. obtuse angulata, sutura tantum superne marginata, postea profunda.

3. Z. algirus LIN. (1758): t. corneo-lutescens vel sulphureoviridula; anfr. 6 , supremi plani, ultimi convexi; diam. maj. 40-45, min. 35-40, alt. 19-41 mm. (Gallia merid., Sicilia, Sardinia, Corsica, Ital. merid., Grcecia, Turcia.) T. subtus nitida, lævis, apert. late oblique lunaris. Syn. Helix oculus capri Müll., 1774. H. agophthalmos Gmel. 1788. Fig. Rossm. Icon. f. 147. 
It Testa superne confertim costulato-striata, striis spiralibus sub lente minutissimis regularibus (:t. non gramulata, tantum costulis transverse striata).

*) Testa spira sat concexa.

4. Z. croaticus Partsch, Fér. (1821): t. obtuse angulata, sutura superne acute marginata. deinde profunda, immarginata; diam. maj. 28, min. 25, alt. $13 \mathrm{~mm}$. (Croatia.) T. cornea, subtus albida, lutescens v. virescens, nitida, anfr. 7. ultimus utrinque convexus, medio albido-cinctus, apert. oblique rotundato-lunaris. Fig. Rossm. Icon. f. 151.

- var. transiens Mouss. (1859): t. paullo minor, depressior, fascia cornea destituta, subtus convexior, cornea, radiis latis albidis ornata; diam. maj. $22-24 \mathrm{~mm}$. (Epinus.) Nonne Z. carniol. proxima? (Möllend.)

5. Z. gemonensis Fér. (1819): t. acute angulata, sutura usque ad aperturam acute marginata; diam. maj. 18, min. 16, alt. $7 \mathrm{~mm}$. (Friaul, Tirolia.) 'T. orbiculato-convexa, lutescens, subtus glabra, nitida, anfr. 6, planiusculi, apertura angulato-lusata. Syn. Hel. isodoma Jan. Fig. Rossm. Icon. f. 153.

**) Testa spira depressa, lineis spiratibus subtilissimis.

6. Z. compressa Z. ap. Rosss. (1836); t. obtuse angulata, supra dense costulato-striata, cinereo-rufescens, sericina, subtus nitida, cinereo-albidoque radiata; diam. maj. 32, min. 2S, alt 12 mm. (Camiolia, Calabria, Croatia.) T. depresso-orbiculata, sutura primum superficialis et acute marginata, demum profunda et immarginata, anfr. 6, convexiusculi, apert. oblique lunaris. Fig. Rossm. Icon. f. 150 .

tt+ Testa transversim rugosa, lineis spivalibus subargutis sed irregularibus (: irregulariter gramulata.)

*) Testa spira globoso-clevata.

7. Z. verticillus FÉr. (1819): t. luteo-fusca, strigis latis remotis luteis ornata, subtus glabra, nitida, virescenti-lutea; diam. maj. 33, min. 29, alt. $19 \mathrm{~mm}$. (Bohem. ad Brandeis, Bararia ad Schellenberg et Passau, Austria, Styria, Carniolia, Carinth. Croatia, Bosna, Gracia.) T. subsemiglobosa, anfr. 7, omnes convexi, ultimus rotundatus, vix conspicue obtuse angulatus, sutura superne marginata, apertura lunato-subcircularis, intus albo-callosum. Fig. Rossm. Icon. f. 149. (Foss.)

**) Testa spira depressa.

8. Z. albanicus Z. ap. Rossm. (1836): t. major, vix conspicue 
angulata; diam. maj. 37, $\min .33$, alt. $14-15 \mathrm{~mm}$. (Da7m., Albania, Morea, Bosna.) T. discoideo-convexa, corneo-fusca, interdum albido-cincta, circa umbilicum lutescens, nitida, anfr. 6, sat convexi, sutura sat profunda, apert. oblique lunaris, marginibus convergentibus. Fig. Rossm. Icon. f. 148.

9. Z. carniolicus A. Schm. ap. Prr. (1959); t. minor, obtuse carinata; diam. maj. 23, min. $201 / 2$, alt. $8 \frac{1}{2}$ mm. (Carniol., Carinth., Bosna, Serbia, Croatia.) T. convexo-depressa, fuscentifulva, subtus nitida, albida, anfr. $6-61 / 2$, vix convexiusculi, sutura marginata, apertura parum obliqua, subangulato-lunaris.

Subfam. Calcarina. Corpus ut in Vitrinina. - Testa crassa, solida, opaca, cretacea, globosa, lavigata, peristomati labiato vel incrassato.

Gen. Leucochroa Веск.

Animal magnum, grosse nugosum, disco lato; orificium respirationis latere dextro supra collare, genitalis ad basin colli; sagitta amatoria ejusque bursa desunt; porus mucosus in apice pedis nullus. Epiphragma crassa, calcarea.

Testa sapissime obtecte umbilicata; anfractus $5-5^{1} / 2$, regulariter accrescentes superiores carinati, embryonales lavissimi, nitentes, ultimus antice descendens; apertura lunatorotundata, margine columellari calloso dilatato, umbilicum in adultis sapissime tegente.

Syn. Helix Drap. et alior.; Leucochroa Beck pr. p, 1837.

Habitat hoc genus semper in locis aridissimis, in campis quam maxime sterilibus, ubi in cavulis vivit; raro in scopulis et rarissime in truncis arborum ascendit.

*) Testa ecarinata.

1. I. candidissima DRP. (1801): t. globosa, cretacea, lrvigata; anfractus sensim accrescentes; diam. maj. 20. min. 17, alt. 13-14 mm. (In litor. mediterr. Gallia, Italice, Sicilia et insularum ceter.) T. tenuissime et irregulariter striatula, subperforata v. imperforata, anfr. 5-6 planulati, superi carinati, ultimus convexus, rotundatus, antice omnino ecarinatus, ad aperturam deflexus, apertura parva, rotundato-lunata, subangulata, peristoma simplex, intus subincrassatum. Fig. Rossm. Icon. f. 367, 814 (f. major.) - Variat 1) rimosa J $\mathrm{JN}_{\mathrm{N}} 1832$ (subcarinata Bourg., umbilicata MкE), t. plus minus umbilicata; 2) microstoma Mке 1831, t. apertura minuta; 3) minor: $t$. diam. 15, alt. $10 \mathrm{~mm}$. (Formæ majores :diam. 25, alt. $20 \mathrm{~mm}$. in Algeria vivunt.) 
2. L. bætica Rossm. (1853): t. globosa, albo-cinerea, superne carioso-rugulosa; anfr. lentissime accrescentes; diam. maj. 25, min. 22. alt. $15-16 \mathrm{~mm}$. (Hisp.) T. exumbilicata, anfr. 51/2, superi carinati, penultimus desuper spectus ultimum latitudine æequaus, ultimus carina sensim evanescente rotundus; apert. perobliqua, late lunata, extus subangulata, peristoma sublabiatum. Fig. Rossm. Icon. f. 812, 813.

*) Testa carinata.

3. L. cariosula Mich. (1833): t. subsemiglobosa, supra cariosa, subtus planiuscula; apertura lunata; diam. maj. 18, min. 16, alt. 10-12 mm. (Hisp.) T. superne globoso-convexa, plus minus visibile spiraliter lineata, usque ad aperturam olotuse carinata, sutura tuberculata, anfr. $5^{1} / 2$, lentissime accrescentes, planiusculi, ult. antice forte deflexus, apertura lunata, peristoma sublabiatum, angulatum. Fig. Rossm. Icon. f. 561. Variat depressa: sublentiformis, utrinque subrequaliter convexa, medio filiformi carinata.

4. I. chionodiscus PFr. (1856): t. conoideo-lentiformis, rugoso-striata, subtus turgidula; apert. rhombeo-lunaris; diam. maj. 26, min. 23, alt. $13 \mathrm{~mm}$. (Tauria.) T. nivea, anfr. 5, ultimus antice breviter deflexus, infra suturam linearem turgidulus, basi convexus, apertura diagonalis, peristoma margini dextro recto, hasali subarcuato. Fig. Mal. Bl. 1856, t. 2 f. $12,13$.

\section{Fam. 3. Helicidæ.}

Maxilla perpendiculariter costata, margine crenato. Uncini radula rquales, bi-tri-cuspidati, breves, laterales secundi ordinis basi verevsa, transrersaliter affixa, quam cuspides plerumque multo majori.

Subfam. Arionina. Corpus per totam longitudinem cum pede conjunctum, postice convexum, orificio respirationis antemediano; forea caudatis mucipara. Teste interne loco granule calcuree plus mimus conglomerate a clypeo gramuloso obtecte (Arion) rel testa interna solida (Geomalacus).

Gen. Arion Férussac.

Animal antice et postice angustius, canda postice elongato-triangularis, forea raudali coquati, sed magis elongata; clypeus postice late rotundatus rel subtrumcatus; orificia respirationis et genit. magis antica.

Testa internce Toro gramule calcarea rel rarissime testa interna submembranacea gramulis conspersa. 
Syn. Limax Auctr. antiqu. pr. p. - Avion Férussac, 1819.

Habitat in silvis frondosis, humidis et umbrosis, sub folia putrida, in agaricis et truncis putrescentibus.

1. A. impericorum Fér. (1819): corpus animalis maturi unicolor, grosse squamosum, magnum, solea æquali latitudine, antice et postice æque rotundata; long. ad 130-150, lat. 18 -25 mm.

(Europa.) Granulæ calcareæ sub clypeo testæ loco; uncinus radulæ medius cuspide utrinque unituberculato, uncini marginales apice secundario externo; sudor non vel leviter coloratus. Sunt præsertim tres colores sub quibus hoc animal demum occurrit: 1. ater Lin. (1758), corpore aterrimo, subtus pallido, limbo sæpe rufo lineis transversis striato (præs. in Europa boreali): 2. rufus Moq.Tand. (1855), corpore miniato vel coccineo, limbo striato, subtus pallido (præs. in Europa meridionali); 3. albus Lin. (1767), corpore toto albo (vel flavido, interdum tentaculis et limbo striato coloralis). Nulti sunt inter illas tranciti colorum et variationes (ex. gr. fulvus, obscure rufus, fusco-castaneus et aliæ), permultæ autem colore formæ animalium crescentium a pullo semper albido vel pallide virescente, omnes fere ab auctoribus ut species descriptæ, quarum secquentes principes: $\boldsymbol{A}$, melanocephalus F. Big., 1822 (L. flavus Müll. ?, Nilss., A. flavus Fér., Lehm.), albidovirescens v. pallide thalassinus, capite et tentaculis atris; $A$. succineus Müll. 1774, rufo-fuscus v. succini coloris, tentaculis nigris; $A$. campestris Mab. 1868, aurantiacus, limbo non striato flavido, punetis crebris aurantiacis ornato (Gall.); A. hibernus Nab. 1868, obscure purpureus, solea albida, limbo transverse brunneo-lineolato (Gall.); $\boldsymbol{A}$. gaudefroyi Mab. 1870, dorso griseo-rufescens v. flavidus, solea medio pallida, limbo griseo atro-lineato (Gall.); A. virescens Mill. 185t, virescens, fasciis duabus lateralibus flavido-aurantiacis (Gall.); A. brunnens Lehm. 1862, caffeatus vel ferrugineus, dorso obscuriore (Germ. bor. et Bohem.) A. olivaceus Schm. 1856, olivaceo-brunneus, obscurius fasciatus, subtus cinereus, sudore luteo (Germ. et Transsilv.); A. bicolor Broeck 1870, albido-flavidus, utrinque fascia nigra (Belgia). Omnes minores vel parvi (pulli). Aliæ formæ magnitudine animali maturo æquales ut species descriptæ, inter quas: A. sulcatus Morel. 1845, omnino niger, margine radiato, castaneo, long. 15-16 Cm. (Lusitania); A. servainianus Lab. 1870, corpus rufum, postice attenuatum obtusum, squamis vix elevatis, corpore contracto rectangularibus, apud exempl. in spiritu conservata omnino deplanatis (Gallia). - Perraro occurrunt (in Belgia et Germ. ad Eberbach) specimina juniora hujus speciei fasciata (corpore fascia obscura utrinque ornato, clypeo lyriforme signato), in quibus fasciæ cum retate omnino exstinguuntur (teste clar. II. Seibert.)

2. A. subfuscus DRP. (1805): corpus animalis robustum, utrinque fascia continua obscura, magis cylindraceum, dorso postice subcarinatum, magnitudine mediocre, tenuiter squamosum; clypens 
elongatus; solea deorsum sensim attenuata, apice rotundata; long. 40-60, lat. 6-10 mm. (Europa.) Granulæ calcarea conglomeratre sub clypeo; uncini marginales cuspidibus duobus secundariis; sudor uberrimus, luteus; corpus supra fusco-castaneum vel rufo-brunneum, dorso obscuriore, fasciis in adultis obsoletis, limbo transverse fusco-striato. Syn. L. rufus Lin. Fauna Su. 1761; A. fuscus Malm, 1868, A. fasciatus Nilss. 1822, A. cinctus Mörch 186t, A. rufus et subfuscus Westerl. 1871. - Uti species distinetre sequentes me judice colore mutationes descriptæ sunt: $A$. rupicola Mab. 1868, virescens, interdum rufescens, obscure fasciatus, limbo albido, striato (Gall., Germ. occ.); A. aggericola Mab. 1870, roseo-flavescens, nigrescente fasciatus, limbo striato. (Gall.); A. vubiginosus Baud. 1867, flavido-rufescens, violaceo-fasciatus, clypeo obscure-zonato, vix granuloso. (Gall.); A. dupuyianus Bourg. 1861, subcoerulescens, clypeo (non abdomine) utrinque fasciato, dorso postice distincte albo-carinato (Gall.); A. bourguignati Mab. 1868, griseo-albidus, utrinque obscure fasciatus, postice non angustatus, dorso in junioribus postice forte, in adultis vix, carinatus, limbo striato (Gall.); A. neustriacus $\mathrm{Mab} .1868$, griseo-rufescens, limbó non striato, de cetero ut præcedens (Gall.); $A$. paladilhianus Mab. 1870, virescens (Gall.); A. citrinus Westerl. 1873, citrinus, dorso vix obscuriore, efasciatus, capite et tentaculis nigricantibus (Suec.) Distinctiores, agre tamen species bonæ, sunt: A. mabillianus Bourg. 1866, cylindricus, antice validus, postice attenuatus, ochraceo-luteolus, cum tribus zonulis (una dorsali) luteo-castaneis longis, dorso valide rugosus, limbo argutissime fusco-sulcato; long. $82 \mathrm{~mm}$. (Gall.); $\boldsymbol{A}$. limacopus Westerl. 1871, rufo-brunneus, dorso obscuro, lateribus pallidis, clypeo irregulariter striis rugosa, solea sulcis 2 longitudinaliter tripartita, partibus lateralibus lineis transversis, parte mediana subelevata irregulariter partita; long. $40-50 \mathrm{~mm}$. (Suec.); A. timidus Morel. 1845, fusco-nigricans, margine lutescente radiato, linea castanea bipartito, clypeo parvo vermiculato, tentaculis parvis, sæpius semireductus, vel fusco-virescens, margine ferreo, capite tentaculisque nigris (Lusit.).

3. A. hortensis FÉR. (1819): corpus animalis elongatum, utrinque fascia discontinua obscura, magnitudine parvum, squamis semicylindraceis juxtapositis obtectum; clypeus brevissimus; solea deorsum forte attenuata; long. 40-50, lat. 4-5 mm. (Europa.) Testa interna membranacea, granulis calcareis conspersa, uncini raIulæ fere ut in A. subfuscus, sudor parcus albus, corpus supra albidum v. pallide cinereum, dorso obscuriore, tentaculis et fasciis nigricantibus, limbo absque lineis transversis, - Plures huic nimis affines formæ ut species descriptæ sunt, a quibus sequentes referam: $A$. pelophitus Mab. 1870, ater, fasciis lateralibus obscurioribus, limbo rufo (Gall.); A. anthracius Bourg. 1866, gracilis, minor (long. c. $30 \mathrm{~mm}$.), uniformiter aterrimus, limbo paullo pallidiori (Gall. merid.); $A$. distinctus Mab. 1868, minor, grisco-flavidus (Gall); A. oresicecus Mab. 
1870, flavescens, tentaculis nigris (Gall.); A. leucophaus Norm. 1852, griseo-coerulescens, maculis et fasciis nigris. (Gall., Belg.)

Species mihi ignote: A. krynickii KaL., Ukraine. A. fuligineus Morel., Lusitania.

\section{Gen. Geomalacus Altmann.}

Animal latitudine aquali, cauda postice rotundata, foven caudali angusta transversali; clypeus vermiculato striatus, postice ovalis; orificia respirationis et genit. minus antica, submediana.

Testa interna solida, lavis, mucleo et striis privata, ovalis, depressa.

Syn. Limax Morel. 1845. - Geomalacus Alum. 1846.

Habitat in muscis et aliis plantis infra scopulos ad radices montium.

1. G. maculosus ALur. (1846); niger, maculis albidis, in clypeo rotundatis in corpore elongatis, ornatus; long. 50-60, lat. $10 \mathrm{~mm}$. (Irlandia occ.-merid. ad Kilnarney, Hisp. in Asturia.) Solea longitudinaliter subæqualiter sed parum distincte tripartita. Cel. Heynem, tres colore formas distinxit: allemanni, ater vel obscure brunneus, albomaculatus; typica, ater maculis flavidis; verkrïzeni, griseus, albomaculatus. Fig. Mal. Bl. XXI. t. 1 f. 1-6. - Celeberr. MoRELET speciem Limacum, quam $L$. anguiformis nominavit et quam cel. Heynem. (an merito?) ad genus Geomalacus duxit, his verbis descripsit: L. fusco-virescens, corpore cylindraceo, utrinque nigro-fasciato, rugis tenuibus striatim reticulato, clypeo elongato, depresso, lævi, atomis nigris notato, capite tentaculisque coeruleis, sub clypeo sæpius contractis, cavitate branchiali antica. Habite la Serra de Monchique en Algarve." - Celeberrimi auctores gallici Normand, Mabille, Baddon et Letounneux sex species e Gallia nominaverunt (G. intermedius N., bourguignati M., paladilhianus M., moitessierianus M., mabilli B., vendeanus L.) quas omnes clar. Heynemann (Malac. Blätt. XXI.) demonstravit a speciminibus junioribus generis Arion creatas esse.

Subfam. Helicina. Animal gracile, inclusum, pallio carnoso collum ad basim circumdante et tunicam membranaceam reliquum corpus tegentem efformante munitum; tentacula-quattuor, superiora majora, apice capitulifera, oculifera; pes oblongus; orificium respirationis in parte superiore postica marginis pallii, orificium genitale in dorsi dextera parte.

Testa forma pervariabilis (planorbica, de- 
pressa, globosa, conica vel elato-turrita) apertura transversa, obliqua, lunaris rel subrotundata.

Gen. Helix Linné.

Genus characteribus subfamitic gandet.

1. Patula Heud. Testa aperte umbilicata depressa (N:o 13 turbinata); anfr. lente accrescentes scepissime costulatostriati; apertura lunari-rotundata, peristomate simplici, recto et acuto.

Habitat sub lapidibus sæpium, sub cortice arborum, in trincis putrescentibus, in muscis et ruderatis, in muros et inter folia putrida, sæpissime socialiter. - Fossilis in stratibus oligocen., miocenico infero, pliocen. infero et pleistocen. toto.

+ Testa lenticulari-depressa, anfractu ultimo carinato; diame. $r-9$, alt. $2^{1 / 2}-3 \mathrm{~mm}$.

1. H. solaria MKE (1830): t. cornea, rufo-maculata, acute carinata; diam. $6-7$, alt. $1 \frac{1}{2}-2 \mathrm{~mm}$. (Silesia, Austria, Carinth., Carniolia, Transsilv., Bosna, Croatia, Sertia, Dalm., Lombardia, Bavaria merid.-austr.) T. latissime et profunde umbilicata, superne planiuscula, subtus excavata, tenuis, dense costulatostriata, raro unicolor, anfr. 6, supra plani, ultimus subtus ad umbilicum altus, intus declivus, sutura perlevis, apertura securiformis. Syn. H. perspectiva Mühlf. (1818), non Say. Fig. Rossm. Icon. f. 353. (Foss.)

2. H. frivaldskyana Rossm. (1842): t. albido-straminea, obtuse carinata; diam. 9, alt. $3^{1 / 2} \mathrm{~mm}$. (Rumelia.) T. perspective umbilicata, subtiliter costulato-striata, anfr. 5 convexi, sutura perprofunda separati, apert. oblique lunaris. Syn. H. convexu Fér. 1819, nee. Rafin. Fig. Rossm. Icon. f. 691.

(H. rotundata Müll. var.)

ti Testa convexo-depressa (N:o 13 turbinata), anfractu. ultimo rotundato.

*) Testa diam. 9-13, alt. 5- $7^{1 / 2}$ mm.

3. H. flavida ZGI, ap. Rossm. (1839): t. plicato-costulata; anfr. $6 \frac{1}{2}$, convexi; apert. lunata; diam. 10, alt. 51/2 mm. (Sicilia.) 'J. flavescens, depressa, suborbiculata, aperte umbilicata, arctispira, sutura profunda, apertura subverticalis, intus levissime callosa. Syn. H. striolate Phil. 1836, nec C. Pfr. H. teresa Ben. nec Alb. Fig. Rossm. Ieon. f. 610 .

4. H. erdelii Rоти (1855): t. confertim chordato-costulata; anfr. 51/2, convexi; apert. late lunaris; diam. 9, alt. $4 \frac{1}{2} \mathrm{~mm}$. (Rhodus.) T. corneo-lutescens, diaphana, late umbilicata, spira con- 
vexa, anfr. lente crescentes, sutura profunda, apert. obliqua, intus levissime labiata. Fig. Roth diss. t. 1. f. 4, 5, 20.

5. H. sudensis Prr. (1846): t. confertim árgute capillaceocostulata; anfr. 6, teretiusculi; apert. late lunata; diam. 13, alt. 71/2 mm. (Candia ad Suda.) T. corneo-lutescens, convexo-orbiculata, perspective umbilicata, anfr. subcylindracei, ult. major, sutura excavata, apert. fauce profunde coerulescenti-callosa, peristomate flexuosorepando. Fig. Rossm. Icon. f. 901.

**) Testa diam. 6-8, alt. 3-4 mm.

6. H. rotundata MüLu. (1774): t. costulata, brunnea, ferrugineo-maculata; diam. 6-7, alt. $2^{1} / 2-3 \mathrm{~mm}$. (Europa.) T. depressa, flavido-brunnea, maculis rufo-brunneis transversis supra obsita, infra cornea, unicolor, rarissime anfractu ultimo fasciis pluribus rufis transversis ornato, spira vix convexa, perspective umbilieata, anfr. 6-7, angnsti, planulati, ultimus superne obtuse angulatus, subtus convexus, sutura profundiuscula, apertura obliqua, depresso-subovatoiunaris, vel angulato-lunata, rotundata. Fig. Rossm. Icon. f. 454. (Foss.) - Variat albina (testa alba vel subvirescens, concolor) \& grisea (t. grisea concolor). tannia.)

- var. pyramidalis JEFFR. (1862): testa subconoidea. (Bri-

- var globosa Fried. (1864): t. minor, convexa, spira elata, anfractu ultimo utrinque rufomaculata. (Hamburg.)

- var. turtoni FleM. (1828): testa depressissima, spira fere plana. (Brit., Gall., Gerin.)

7. H. ruderata STUd. (1820): t. dense costulato-striata, lutescenti-cornea, concolor; diam. 7-8, alt. 3-4. (Eur., in region. montosis.) T. convexa, perspective umbilicata, anfr. 5, convexi, sat accrescentes, ultimus rotundatus; v. obsolete subangulosus, sutura profunda, apert. perobliqua, lunato-ovalis. Fig. Rossm. Icon. f. 455. (Foss.) Obs. Ex. spuria hujus et præcedentis in valle Reposoir in Alp. Savoy. celeb. Dumont et Mortillet observarunt.

8. H. carpetana HiD. (1870): t. supra vix striatula, infra lævigata, subvirenti-cornea, unicolor; diam. 6, alt. $3 \mathrm{~mm}$. (Hisp. in montib. Carpetanis.) T. late umbilicata, orbiculato-depressa, spira convexa, anfr. 5, convexiusculi, subangusti, ultimus paullo major, rotundatus, sutura minus profunda, apert. rotundato-lunaris.

***) Testa diam. 3-31/2, alt. $1^{1 / 2}-2 \mathrm{~mm}$.

a) Testä depressa vel globoso-depressa.

9. H. sororcula BEN. (1859): t. albido- fulvescens, confertim costulata, opaca; apert. subovato-lunata, non labiata; diam. $3 \frac{1}{2}$ alt. $1^{3} / 4 \mathrm{~mm}$. (Sicilia.) T. umbilicata, anfr. 4, convexiusculi, ultimus 
non dilatatus, apert. parum obliqua, peristoma marginibus conniventibus, pariete subincrassato. Fig. Ben. Ill. Sic. t. 4 f. 16.

10. H. tenuicostata Shutru. ap. PFr. (1846): t. cornea, tenuiter costulata; apert. lunato-circularis, non labiata; diam. $23 / 3$, alt. $1^{1 / 3} \mathrm{~mm}$. (Sardinia; Lusitan. ad Oporto: Morel. ex. sp.) T. perspective umbilicata, depressa, tenuis, anfr. 4 , convexiusculi, ultimus basi sublævigatus, apert. subobliqua, peristomate simplici acuto.

11. H. aranea PARr. ap. Pfr. (1870): t. cerea, confertim subcordato costulata; apert. lunato-rotundata, non labiata; diam. $2 \frac{2}{3}$, alt. $1 \frac{1}{3} \mathrm{~mm}$. (Sicilia.) T. apert. umbilicata, depressa, tenuis, anfr. 4, convexiusculi, ultimus subdepresso-rotundatus, apert. parum obliqua, peristomate simplici, recto.

12. H. hauffeni F. Scru. (1855): t. lutescenti-alba, subdiaphana, eleganter chordato-costata; apert. diagonalis, subsinuato-rotundata; diam. $31 / 4$, alt. $1 \frac{1}{2} \mathrm{~mm}$. (Carniolia in cavernis.) T. sublate umbilicata, depressa, anfr. $4^{1} / 2-5$, convexi, ultimus major, teres, apertura margine basali leviter labiato, anguste reflexo. Fig. Journ. Conch. XI. t. 13. f. 4.

\section{ß) T. turbinata.}

13. H. rupestris DRP. (1801): $t$. depressa turbinata, fuscocornea, subtilissime striata; apert. rotundata, non labiata; diam. $21 / 2-3$, alt. $1 \frac{1}{2}-2 \mathrm{~mm}$. (Europa media et merid.) T. sericina, anfr. 5, convexi, subcylindracei, angusti, ultimus rotundus, sutura profunda, apert. peristomate simplici, acuto, recto. Syn. H. umbilicata Mont. 1803, $H$. aliena Z. ap. Pfr. 1841. $H$. spirula Villa 1841. Fig. Rossm. Icon. f. 534. - Variat depressa, umbilico latiori (H. saxatilis Hartm.) et elevata, trochoides, umbilico angustiore ( $v$. rupicola Stab.) ****) Testa diam. 11/2-2, alt. $1-1^{1 / 4} \mathrm{~mm}$.

a) Testa tota striata.

14. H. pygmæa DrP. (1801): t. minutissime striatula, spira convexa; anfr. convexiusculi, sutura impressa; diam. $2 \mathrm{~mm}$., alt. 11/ mm. (Europa.) T. late pervio-umbilicata, cornea, spira obtusissima, anfr. $4^{\mathrm{T}} / 2$, lente et regulariter accrescentes, ultimus non descendens, apert. vix obliqua, late lunato-rotundata. Fig. Rev. \& Mag. 1863. t. 18. f. $1-4$. (Foss.)

ק) Testa lavis.

15. H. massoti BourG. (1863): t. lævigata, compressa, supra fere complanata; anfr. convexi, sutura valde profunda; diam. $11 / 2$, alt. $1 \mathrm{~mm}$. (Pyrencece orient. et Catalogne.) T. late pervio-umbilicata, pallide cornea, spira fere plana, anfr. lentius et magis regulariter accrescentes, ultimus leviter descendens, apert. obliqua, valde lunata, rotundata. Fig. Rev. \& Mag. 1863. t. 18. f. 5-8. 
r) Testa lamellata.

16. H. microplouros $\mathrm{PAG}_{\mathrm{AG}}(1854)$ : t. oblique lamelli-costata, supra planiuscula; anfr. sat celeriter crescentes, ultimus rotundatus, non descendens; apert. oblongo-rotundata; diam. 2, alt. 1 mm. (Gall. media et Pyren. orient.) T. aperte pervio-umbilicata, cornea v. succinea, spira vix convexa, anfr. 4, convexiusculi, ult.infra convexo-rotundatus, apert.' obliqua, parum lunata. Fig. Rev. \& Mag. 1863. t. 18 f. 9-13. - Obs. H. temuicostatce simillima!

17. H. elachia Boung. (1863): t. lamellicostata, supra planata; anfr, rapide accrescentes, subangulati, ultimus lente descendens, ad umbilicum subcarinatus; apert. late ovato-rotundata; diam. $1 \frac{1}{2}$, alt, $3 / 4 \mathrm{~mm}$. (Gallia, in valle fluvii Loire.) T. umbilicata, pallide cornea, hyalina, anfr. $3^{1} / 2$, sutura impressa separati, apert. ampla, parum lunata. Fig. Rev. \& Mag. 1863. t. 18. f. 14-17.

H. servaini Bourg. (1869) - Gallia, in valle fluvii Seine - est mihi ignota species.

\section{d) Testa anfractu ultimo striata.}

18. H. simoniana Bourg. (1870): t. compressa, anfr. ultimo sub validissima lente striatulo; anfr. convexi, lente accrescentes, ultimus vix major; apert. fere rotundata, lunata; diam. 1, alt. $3 / 4$ mm. (Gall. circa Toulouse.) T. late pervio-umbilicata, luteo-cornea, anfr. $3^{1 / 2}-4$, sutura perprofunda, apert. vix obliqua. Fig. Bourg. Moll. nouv. fase. 11,12 .

Obs. H. dibenedictiana Calc., cupaniana Calc., brocchiana Calc., schwerzenbachiana Calc., templorum et bocconiana Ben., teste amiciss. Kobelt, et H. assarinensis Calc. me judice, omnes e Sicilia, excludendæ sunt. (Cfr. Jahrb. 1875 p. 10.)

2. Acanthinula BEck. Testa perforata, globosa ad turbinata, epidermide brumea, vel membranaceo-costulatoplicata vel aculeata, induta; anfr. $4-6$ teretes, celeriter accrescentes; apertura rotundata, peristomate tenui, expansiusculo, marginibus approximatis.

Habitat in silvis frondosis inter folia putrescentia, sub lapidibus et ad radices arborum, plerumque prope aquas.

19. H. lamellata JEFFR. (1830): t. conoideo-globosa, sericina, flavido cornea, membranaceo-costulata; apert. depressa, lunaris, non labiata; diam. 21/3, alt. 2 mm. (Britan., Dan., Suec., Germ. bor.) T. tenuis, anfr. $6^{1} / 2$, subæquales, sutura profunda, angusta, peristoma simplex, acutum, rectum, margine columellari reflexiusculo. Syn. H. scarburgensis Rossm. 1838. Fig. Rossm. Icon. f. 533. 
20. H. aculeata MüLL. (1774): t. globoso-turbinata, opaca, fusca, costis lamellatis transversis medio in spinas elongatis prædita; apert. rotundata, intus submarginata; diam. $2-21 / 2$, alt. 2-31/4 mm. (Europa.) T. tenuis, anfr. $4^{1} / 2$, convexi, subcylindracei, ultimus maximus, sutura profunda, apertura parum lunata, peristomate expansiusculo, membranaceo, obscuro, intus pallide labiato, marginibus valde approximatis. Fig. Rossm. Icon, f. 526. Variat allida.

- var. sublavis Westerr. (1871): t. costis obsoletis, anfr. 4, labro roseo, spinis destituta. (Succia, Dania.)

21. H. harpa $S_{A Y}$ (1824): t. ovato-turrita, sericina, virescens vel corneo-lutescens, confertim striatula et distanter membranaceo-costulata; apert. rotundato-sublunaris, non labiata; diam. $2-21 / 2$, alt. $31 / 2-4$ mm. (Suec.bor., Norveg., Fennia.) T. tenuis, anfr. $4-4 \frac{1}{2}$, convexiuseuli, ultimus ventrosus, sutura profunda, apert. sæpe semi-ovalis, peristomate simplici, acuto, margine columellari subreflexo. "Animal ovoviviparum, marginibus pedis profunde crenulatis" (Morse.) Syn. Pupa costulata Migh. 184t, Bulimus harpa Pfr. 1847, H. aculeata Nord. \& Nyl. 1856, H. amurensis Gerstf. 1859, Zoögenetes harpa Morse 1864. Fig. Portl. Soc. Nat. Hist. I. t. 1. f. 1-12.

3. Trigonostoma Fitz. Testa umbilicata (in N:s 36 \& 40 obtecte perforata), orbiculata, depressa, arctispira; aperture obliqua, anguste lunaris, angulata vel simuata, peristomate reflexo, labiato, sape calloso.

Habitat sub lapidibus, in rimis murorum vel rupium, in truncis arborum putridis, inter folia putrescentia, sub cortice arborum veterum etc. Fossilis in strato oligocen, miocen. infero, pliocen. et pleistocenico.

\section{+ Testa lenticularis, acute carinata.}

*) Peristoma superne rectum, margine basali incrassato vel reflexo, edentato.

22. H. lenticula FÉr. (1821): t. subtilissime costulato-striata; anfr. $4 \cdot 1 / 2-5$, ultimus major; apertura late lunaris, peristomate flexuoso; diam. 8, alt. 3 mm. (Gall. merid., Pyrence, Hisp., Lusit. merid., Dalm., Sicil., Gracia.) T. carinata, late umbilicata, fusco-cornea v. flavescens, opaco, supra convexiuscula, infra convexa, apert. marginibus eallo tenuissimo junctis, columellari reflexiusculo, subtiliter limbato, ad carinam obtuse angulata. Fig. Rossm. Icon. f. 452 .

23. H. lens FÉR. (1821): t. arctispira, costulato-striata, rarissime pilosa; anfr. 7 , subæquales; apertura angusta, subsecuri- 
formis, margine dextro et basali reflexis; diam. 13, alt. $6 \mathrm{~mm}$. (Gracia.) T. argute carinata, aperte umbilicata, fusco-cornea, opaca, supra subplana, infra ventrosa, costulis deusis, subrectis vel levissime curvatis, apert. callo punctiformi juxta insertionem marginis superi, peristomate albo, leviter labiata. Syn. H. barbata Desh. 1836. Fig. Rossm. Icon. f, 450 .

- var. lentiformis $Z_{\text {.: }}$ t. minor, umbilico angustiore, carina minus acuta, apertura angusta sublunaris; diam. $9-10$, alt. $3^{1} / 2-4$. mm. (Macedonia, Thessalia, Attica.)

- var. aliostoma mh.: t. angustius umbilicata, nitida, costata, costis sat distantibus, arcuatis vel subgeniculatis, apert. subtetragona v. subrhomboidea, marginibus leviter reflexis; diam. 13, alt. $6 \mathrm{~mm}$. (Corfu: Parr. sub nom. H. barbatæ.) T. cornea, supra convexa, infra ventrosa, apertura callo punctiformi juxta insertionem marginis superi, margine columellari et $\mathrm{m}$. exteriori breviore cum basali angulos subrectos formantibus. Forte species distincta.

24. H. lenticularis Mor. 1853): t. costulato-striata; anfr. 7, lente accrescentes, ultimus ad carinam compressus; apertura angulato-lunaris, margine basali albido, reflexo, crasso; diam. 17, alt. $6 \mathrm{~mm}$. (Hispan. circa Tarifa.) T. acute carinata, umbilicata, corneo-fulva. Fig. Journ. Conch. 1853. t. 5. f. 13, 14.

25. H. calpeana Mor. (1854): t. arctispira, tenere costulatostriata; anfr. $7^{1} / 2$, subæquales; apertura securiformis, margine basali sinuoso, reflexo; diam. 11, alt. $5 \mathrm{~mm}$. (Hisp. prope Gibraltar.) T. medio acute carinata, utrinque subæqualiter convexiuscula, tenuis, pervie umbilicata, corneo-fulva, anfr. supra complanati.

\section{*.*) Peristoma expansum vel reflexum, margine basali dentato.}

26. H. barbula Charp. ap. Rossm. (1838): t. confertim subtilissime costulata; apert. angusta, lunato-trisinuata, peristomate basi replicato, albo-labiato, bidenticulato; diam. 11 , alt. $4 \frac{1}{2} \mathrm{~mm}$. (Lusitania.) T. aperte umbilicata, cornea, arctispira, anfr. 6 planulati, ultimus antice constrictus, subtus convexus. Syn. Carocolla guerini Ant. 1839. Fig. Rossm. Icon. f. 451. Carina variat obtusior, immo interdum obsoleta, teste celeb. Morelet.

27. H. rangiana FE̊R. (1819): t. confertim radiato-striata; apertura depresse lunaris, peristomate ad carinam uncinatim recurvato, margine basali incrassato, reflexo, unidentato, extus scrobiculato; diam. 12, alt. $5 \mathrm{~mm}$. (Gall., Pyren. orient. et Provence.) T. aperte umbilicata, cornea, anfr, $6^{1} / 2$, vix convexiusculi, lente 
accrescentes, ultimus subtus turgidulus, sutura impressa, margo columellaris postice denticulo secundario. Fig. Rossm. Icon. f. 537.

28. H. turriplana Mor. (1845): t. densissime granulata; apertura depressa, trisinuata, peristomate replicato, calloso, albolabiato, bidenticulato, extus impressionibus duobus signato; diam. 13-16, alt. $4 \frac{1}{2}-5 \mathrm{~mm}$. (Lusitania.) T. umbilicata, pallide cornea (raro ferruginea), superne acute carinata, carina utrinque sulco terminata, anfr. 6 marginati, plus minus exserti, subplani, ultimus subtus turgidus. Fig. Rossm. Icon. f. 537.

if Testa depressa, arctispira, ecarinata (anfr. ultimus rotundatus vel superne obtuse angulatus.)

*) Peristoma expansum rel plus minus reflexum.

29. H. constricta BouB. (1836): t. confertim arcuato-striata, anfractu ultimo superne obsolete angulato, spira parum elevata; diam. $6 \frac{1}{2}$, alt. $3-31 / 2 \mathrm{~mm}$. (Pyrenœœ.) T. anguste umbilicata, fuscula, anfr. $5 \frac{1}{2}$, convexiusuli, lente accrescentes, angusti, ultimus subtus inflatus, pone aperturam constrictus, apert. subverticalis, anguste lunaris, superne sinuosa, peristomate albido, marginibus lamina compressa erecta in pariete junctis. Fig. M. Tand. Hist. t. 10. f. $23-25$.

30. H. nautiliformis Porro (1838): t. lrvis, hispida, anfractu ultimo rotundo, reliquos involvente, spira immersa; diam. $5-6^{1} / 2$, alt. $2^{1 / 2}-3^{1} / 2$ mm. (Lombartia.) T. utrinque, subtus perspective umbilicata, cornea, anfr. 5, superne vix conspicui, apert. subverticalis, anguste lunaris, peristomate reflexo, rubello labiato, marginibus linea callosa tenui in pariete subjunctis. Gen. Drepanostoma Porro, Fig. Rossm. Icon. f. 615.

**) Peristoma angulatim reflexum.

a) Apertura edentula, peristomate calloso.

a) Testa aperte umbilicata.

f) Anfractus ultimus antice non descendens; testa muda.

31. H. Iusitanica PFr. (1841): t. mediocriter umbilicata, discoidea; anfr. $5 \frac{1}{2}$, exteriores precedentibus duplo latiores, ultimus rotundatus; apertura late lunaris; diam. 15 , alt. $5 \mathrm{~mm}$. (Lusitania.) T. fusca, suboblique confertim costulata, spira plana, peristoma album. Fig. Chemn. Cab. Hel. t. 64. f. 10-12.

32. H. tarnieri Mor. (1854): t. mediocriter umbilicata, subdiscoidea; anfr. 7, omnes angusti, ultimus superne obtuse angulatus; apertura depressa, lunaris, parum obliqua; diam. 12, alt. $5 \mathrm{~mm}$. (Tarifa.) T. fusco-cornea, arctispira, confertim costulato- 
striata, spira vix emersa, convexiusculi, ult. non deflexus, peristoma callosum.

33. H. boscæ Hid. (1869): t. anguste umbilicata, depressa, spira plana; anfr. 6, lente accrescentes, ultimus superne obtuse angulatus; apertura anguste lunaris, margine supero brevissimo, basali reflexo infra angulum ultimi anfractus appresso; diam. 11, alt. $5 \mathrm{~mm}$. (Hisp.) T. corneo-fusca, confertion arcuato-striata, anfr. parum convexi, ult. basi convexus, apertura diagonalis, peristomate albido vel carneo, margine supero parum expanso, subemarginato. Fig. Journ. Conch. 1869. t. 2. f. 1.

\section{ff) Anfractus ultimus antice gibbus, deflexus;} testa typice pilosa.

34. H. angigyra Z. ap. Rossm. (1835): t. minutissime punctulata et striolata, superne omnino plana vel concaviuscula; apertura subtriangulato-lunaris; diam. 10-11, alt. $4 \frac{1}{2} \mathrm{~mm}$. (Italia boreal., Helvetia, Tirolia merid.) T. pervie umbilicata, pallide cornea, pilis brevibus densissimis deciduis obsita (his detritis nuda et punctulata), anfr. 6, convexi, arcte convoluti, ultimus major, infra tumidus; apertura perobliqua. Fig. Rossm. Icon. f. 21.*

35. H. corcyrensis Partsch, Desh. ap. Fér. (1821): t. costulato-striata, superne convexiuscula; apertura angusta, lunaris; diam. 10-11, alt. $4 \frac{1}{2}$ mm. (Corfu, Cephalonia, Epirus; Turcia ad Dobrudscha, Varna, circa Eidos.) T. sat anguste umbilicata, pallide cornea, hispida (pilis detritis plus minus nuda), anfr. 7, convexiusculi, lentissime accrescentes, ultimus superne obtuse angulatus, subtus introrsum convexus, apertura parum obliqua. Syn. $H$. contorta Z. ap. Rossm. nec. Fér. Fig. Rossm. Icon. f. 535.

- var cephalonica Mouss. (1859): t. paullo minor, apert. angustior, falciformis, marginibus callo tenui, ad superi insertionem densiori, junctis. (Cephalonia.)

- var. octogyrata Mouss. (1859): t. major (d. 14, a. 6 mm.), supra convexiuscula, summo plano, anfr. $71 / 2-8$, ultimo rotundato. (Grac. in Previsa, Dalm. in Lesina.)

- var. canaliferce ANT. (1839): t, spira elatiore, anfr. ultimus superne magis angulatus, apertura angustiore, peristomate crasso, extus canali angusto cincto. (Epirus, Rumelia, Balkan.)

- var. girva FRIW. (1838): t. minor, apertura coarctata, labro crassiore. (Albania, Balkan.)

b) Testa subobtecte umbilicata. 
36. H. barbata FÉr. (1821): t. costulato-striata, lirevipilosa, depressa; diam. 13, alt. $5 \mathrm{~mm}$. (Candia, Ballan, Gracia in Hymotto.) T. corneo-fusca, anfr. 6, parum convexi, lente accrescentes, ultimus superne obsolete angulatus, antice breviter descendens, basi convexus, apert. diagonalis, subauriformis, margine supero anguste expanso, basali reflexo, antrorsum flexuoso, ad umbilicum dilatato, eum interdum fere claudente.

\section{B) Apertura peristomate dentato.}

\section{a) Testa aperte umbilicata.}

\section{t) Testa obsolete striatula.}

37. H. obvoluta MüLL. (1774): t. pilosa; anfr. $6-61 / 2$; angusti; apertura verticalis, obtuse triangularis, margine dextro et basali obtuse unidentatis; diam. 11-13, alt. 5-6 mm. (Europa ad Daniam.) T. aperte umbilicata, utrinque plana, brunnea, opaca, spira medio sæpius immersa, anfr. convexi, penultimus prominulus, ultimus compressus, antice scrobiculatus, peristoma reflexum, intus roseum vel lilacinum. Syn. H. trigonophora Lax. 1792, H. bilabiata Oliv. 1792, H. holosericea Gape. 1789. Fig. Rossm. Icon. f. 21. (Foss.) - Variat 1) pallida \& 2) dentata, dentibus aperturæe elongatis.

38. H. holoserica STud. (1876): t. holoserica; anfr. $4^{1} / 2-5$, regulariter accrescentes; apert. verticalis, trisinuata, valde bidentata; diam. 10-11, alt. 4-5 mm. (Ricsen-et Eragebirge, Böhmerwald, Alpes, Gall. merid.orient., Hisp. oce.) T. umbilicata, cornea, supra subplana, subtus convexa, parce hrevipilosa, anfr. convexiusculi, ultimus antice breve deflexus, constrictus, scrobiculatus, superne obsolete angulatus, apertura "trifoliata", peristoina latum, angulatim reflexum, carneo- vel albo-labiatum. Syn. H. holosericea Stud., H. diodonstoma Bourg. 1862. Fig. Rossm.'Icon. f. 20. Variat major.

39. H. diodonta MÜHLF. ap. Fér. (1821): t. minutim granulato-striata; anfr. $4^{1} \frac{1}{2}-5$, regulariter accrescentes; apertura subhorizontalis, panduræformis, bidentata; diam. 11, alt. $4 \mathrm{~mm}$. (Bunatus.) T. late umbilicata, discoidea, corneo-fusca, anfr. vix convexiusculi, ultimus subcarinatus, antice deflexus, superne impressus, peristoma reflexum, rubicundum, dentibus 2 oppositis in margine supero et infero munitum. Fig. Rossm. Icon. f. 19.

\section{ft) T'esta lamellato-costulata.}

40. H. triaria FRIW. (1839): t. parva, arctispira; apertura sinuosa, peristomate bidenticulato, pariete lamellosa aut inermi; diam. 4, alt. $2 \mathrm{~mm}$. (Banatus Inngaria, Transsilv.) T. perspective umbilicata, depressa, cornea, eleganter costulata, spira convexa, anfr. 6, convexiusculi, ultimus superne angulatus, antice deflexus, subtus convexus, attenuatus, apertura perobliqua, subhorizontali-triangu- 
lata, peristomate reflexo, labiato. Syn. H. ocskayi Stentz. Fig. Rossm. Icon. f. 611. Variat major: diam. 5, alt. $3 \frac{1}{2} \mathrm{~mm}$.

b) Testa obtecte perforata.

41. H. buvinieri Micr. (1841): t. depressa, costulato-striata; anfr. 7-8, angustissimi; apertura late lunaris, margine basali leviter arcuato, unidentato; diam. 9-10, alt. $4-5 \mathrm{~mm}$. (Hisp. Asturia:) T. cornea, anfr. convexiusculi, ultimus basi convexior, striatulus, apert. subverticalis, peristomate angulatim breviter reflexo, intus albo-calloso. Syn. $H$. gougeti Pfr. (1818), non Terv., H. asturica Pfr. 1854.

4. Triodopsis RAF. T. obtecte perforata; apertura subtriangularis, sinuoso-coarctata, pariete dente valido, oblique intrante munito, peristomate albo-calloso, late angulatim reflexo.

Habitat in silvis sub foliis putridis et in truncis putrescentibus. Fossilis in strato pleistocenico supero.

42. H. personata LAM. (1792): t. lievigata, pubescens; apertura angulato-trisinuata, intus callosa, bidentata, lamella dentiformi in pariete semiclausa; diam. 7-11, alt.4-6 $\mathrm{mm}$. (Gallix, Helv., Illyria, German. media et merid., Italia.) T. cornea, opaca, spira parum elevata, anfr. 5-51/2, convexiusculi, paulatim accrescentes, ultimus antice constrictus, breviter deflexus, apertura trigonolunata, peristomate lato, reflexo, rufescente, marginibus lamina parietali subjunctis. Fig. Rossm. Icon. f. 18. (Foss.)

5. Vallonia Risso. Testa aperte umbilicata, depressula, costata; striata vel lavis; anfr. $3^{1} / 2-4$, regulariter accrescentes; apertura subcircularis; peristoma reflexum, candide labiatum, marginibus conniventibus.

Habitat in hortis, silvis et campis, locis subhumidis sub lapidibus, in foliis putridis, in muscis \&c., vel locis montosis, petrosis, siccis et arenosis, infra muros, sub lapidibus sæpium etc. Fossilis in strato eocen. infero, oligocen., miocen., pliocen. medio et pleistocenico toto.

43. H. pulchella MÜLL. (1774): t. depressa, albida, pellucens, nitida, subtilissime striatula; ànfr. $3^{1} / 2-4$, ultimus antice paullo dilatatus, non deflexus; apertura subcircularis, lunata, marginibus late reflexis, candide labiatis; diam. $2 \frac{1}{2}$, alt. $1 / 3 \mathrm{~mm}$. (Europa.) T. aperte umbilicata, spira obtusa, anfr. convexiusculi, ad suturam subcanaliculatam paullulum angulati, apertura obliqua, marginibus approximatis. Fig. Rossin. Icon. f. 440. (Foss.) 
44. H. costata MüLc. (1774): t. depressa cinereo-albida, subopaca, membranaceo-costulata; anfr. $3 \frac{1}{2}(-4)$, ultimus antice non dilatatus, deflexus; apertura subcircularis, vix lunulata, albolabiata, margine supero expanso, basali reflexo; diam. 3, alt. $11 / 2$ mm. (Europa.) T. subaperte umbilicata, anfr. convexiusculi, sutura angusta sed profundiuscula separati, apert. perobliqua, marginibus fere contiguis. Fig. Rossm. Icon. f. 439. (Foss.)

?. H. adela WesterL. (1874): t. convexa, albida, nitidula, striata; anfr. $4-4^{1} / 2$, ultimus antice non dilatatus, ad aperturam deflexus; apertura ovato-rotundata, lunulata, peristomate non limbato, nec labiato, vix reflexiusculo; diam. $3^{1 / 3}$, alt. $2 \frac{1}{4} \mathrm{~mm}$. (Sibiria, Europa arctica?) T. late unbilicata, anfr. convexi, sutura sat profunda separati, apert. obliqua. Subfossilis forma minor in turfosis submarinis ad Ystad Sueciæ frequenter inventa. Fig. MIal. BI. XXII. t. 2. f. 1-4. (Foss.) H. (pulch.v.) tenuilabris A. Braun (testa pluribus locis Germannix fossilis) proxima, forte eadem species, sed differre videtur testa depressa, anfractubus minus convexis, costulis creberrimis et aliis elatioribus distantibus ornata, anfractu ultimo antice dilatato et marginibus aperturæ reflexis.

6. Petasia (Bk.) M. TAND. T'esta anguste perforata, conoidea vel turbinato-globosa, arctispira, gramulato-striata; apertura obliqua, peristomate intus labiato, subreflexo, dentato.

Habitat in memoribus et silvis frondosis inter folia putrescentia, locis humidis, imo aquosis, juxta rivulos etc. Fossilis in strat. pleistocen. infr. et medio.

45. H. bidens Chems. (1786): t. fulvescenti-cornea, pellucide cingulata; anfr. 6-8; apertura depressa, trisinuato lunaris, labro reflexo, intus bidentato, extus bifoveolato; diam. 10, alt. $6 \mathrm{~mm}$. (Europa precipue orientalis.) T. angustissime et obtecte perforata, tenuissime striatula, subangulata. Syn. $H$. bidentata Gmel. 1788. Fig. Rossm. Icon. f. 14. (Foss.)

- var. minor: t. minor, depressior, cornea, peristomate albo; diam, $61 / 2-8 \%$, alt. $4^{1} / 2-61 / 2 \mathrm{~mm}$.

- var. major Rossm. f. 431: t. rufobrunnea, spira elatiore, peristomate rufescente; d. $10-13$, a. $73 / 4-9 \mathrm{~mm}$. (Banatus.) H. diodon Parr., H. dibotrion Friw.

7. Trichia НАгтм. Testa umbilicata vel perforata, globoso depressa, scepius pilosa; anfr. 5-7, lente accrescentes, convexiusculi; apertura late lunaris vel lunari- 
rotundata, peristomate acuto, brevissime expanso, intus labiato, margine basali reflexo.

Habitat locis umbrosis et subhumidis in silvis et fruticetis, in plantis et fruticibus, rarior inter folia putrescentia et in truncis putridis. Fossilis in stratibus omnibus a str, eocenico supero (exc. pliocen. infero.)

$$
+ \text { Testa pilis longis villosa. }
$$

46. H. villosa DRP. (1805): t. aperte umbilicata; apertura ovato-lunaris, intus remote candido-labiata; diam. 12 , alt. $6 \frac{1}{2}-7$ mm. (Gall., Helv.. Tirol. bor., German. merid. occid., Italia bor.) T. corneo-fulva, cornea vel luteo-pallida, transversim striata et sub lente densissime longitudinaliter rugoso-striatula, tenera, opacá, subpellucida, depressa, spira late convexa, anfr. 6, convexiusculi, lente accrescentes, ultimus penultimo duplo major, subtus subtumidus, antice descendens, peristoma tenue, acutum, vix subpatulum. Syn. $\boldsymbol{H}$. pilosa v. Alt. 1812, H. hispidula. Ján. 1832. Fig. Rossm. Icon. f. 425. (Foss.) Variat 1) rubra, 2) brunnea, 3) minor: t. $10 \mathrm{~mm}$. lata, umbilico angustiore, 4) major: t. $14 \mathrm{~mm}$. lata, $8 \mathrm{~mm}$. alta.

47. H. piotruskyana PARr. ap. Chemn.: t. anguste umbilicata; apertura rotundato-lunaris. vix sublabiatum; diam. 9 , alt. 5 mm. (Podolia, Hungaria.) T. cornea, tenuis, spira convexa, apice obsolete mucronulata, anfr. 5 , convexi, ultimus obsolete angulatus, antice descendens, peristoma simplex, acutum, marginibus subconniventibus, basali arcuato, columellari dilatato, patente. Fig. Chemn. Helix. t. 148. f. 11, 12 .

tf Testa pilis brevibus vel brevissimis, (sæpius caducis) hispida vel muda.

*) Testa late vel aperte umbilicata (sæpius depressa.)

a) Apertura intus albo-labiata.

48. H. rufescens PenN. (1777): t. major, solidula, depressa, sæpissime distincte angulata, spira medio paullulum conoideo-elevata et apice mammillata (var. depresso-globosa), nuda vel subnuda (juvenis parce tomentoso-pilosa); apert. ovato-rotundata vel subrotundato-lunaris; diam. 12, alt. $61 / 2 \mathrm{~mm}$. (Europa media.) T. late umbilicata, pallide cinerea, plerumque rufo tincta et interdum transverse strigata, fascia pallida in angulo cincta, subtiliter et dense irregulariter striata, anfr. 6-7, regulariter accrescentes, ultimus supra planulatus, infra. cenvexus, sutura profundiuscula, apertura ovatorotundata, margine basali extense regulariter arcuato (var. rotundata, margine basali forte arcuato), labio lato albo a peristomate remoto extus conspicuo munita. Syn. H. striolata C. Pfr. 1828. Fig. Jahrb. 1874. t. 8 f. 1. (Foss.) - Variat 1) rufa, 2) alba, et 3) subcarinata Cl. 
Jahrb. 1874. t. 8. f. 6 (t. magis utrinque compressa, adeoque subacute angulata.)

- var. clandestina HARTM. (1821): t. fusca vel griseo-cornea, anfr. ultimus dilatatus, sutura profunda, apertura rotundatolunaris, intus tenue albo-labiata; d. 11 , a. $6 \mathrm{~mm}$. (Helvetia.) Fig. Jahrh. 1874, t. 8. f. 3.

- var. dambialis CL. (1874): t. anguste umbilicata, albida vel pallide cornea, spira subconica, anfr. 6, lentissime et requaliter accrescentes, apertura semilunari-rotundata, intus callo albo prominente prope marginem munita; d. $10-11^{1} / 2$, a. $61 / 2-7 \mathrm{~mm}$. (In sitvis ad Danubium copiosiss.) Fig. Jahrb. 1874. t. 8. f. 4.

-.. var. montana STud., C. Pfr. (1821): t. angustius umbilicata, brunnea, fusca, globuloso-convexa, parcius striatula, anfr. 5 , convexi, lente accrescentes, ultimus rotundatus; apertura subrotundato-lunaris, tenue labiata; d. $10-11$, a. $61 / 2-7 \mathrm{~mm}$. (German. merid.-occ.,Helv. dec., Suec.) syn. H. circinnata Rossm. pr. p. 1835. Fig. Rossm. Icon. f. 12. Jahrb. 1874. t. 8. f. 2. Variat minor: t. parvula (d. 8, a. $5 \frac{1}{2} \mathrm{~mm}$.), spira alta, globoso-conoidea (Itelvetia, Bavar. ad Mariabrunn, unde misit nobilissima Paulueci.)

- var. submontana MAB. (1868): t. aperte umbilicata, corneorufescens vel-corneo-albescens, subgloboso-depressa, sat conferte irregulariter striata et pilis raris ornata, anfr. $5-5 \frac{1}{2}$, subconvexiusculi, sat regulariter accrescentes, ultimus majusculus, compressorotundatus, apert. ovato-rotundata, distincte albo-labiata; d. 91/2, a. 4-5 mm. (Gall. orient., Bavaria.) In Dania (ins. Mïen) amiciss. Poulsen formam invenit et benigne communicavit: t. minor, suborbiculata, anfr. $6-6 \frac{1}{2}$, convexi, lentissime accrescentes, apertura lunata, basi (inter marg. colum. et basalem substrictum) angulata; $\mathrm{d}$. $8 \frac{1}{3}-9$, a. $4-5 \mathrm{~mm}$.

49. H. coelata Stud. ap. Charp. (1837): t. tenuis, perdepressa, obsolete angulata, demum nuda (juvenis pilis brevissimis mox caducis dense obsita); spira subplana; apert. rotundata; diam. $91 / 2$, alt. $4-5 \mathrm{~mm}$. (d. 8 , a. $4 \mathrm{~mm}$.) (Helvet.: Charp., Bavarit: Cless., Carinth. ad Malborgeth et Carniolia ad Laibach: ipse.) T. late umbilicata, pallide cornea, albida vel flavescens, diaphana, subtilissime striata, anfr. 6. lentissime accrescentes, superne planiusculi, infra perconvexi, versus umbilicum subangulati, præcedentes fere verticaliter tangentes, sutura sat profunda, apertura rotundata, prope marginem labio tenui albido, extus vix conspicuo, munita. Fig. Jahrb. 1874. t. 8. f. 8. Huc verosimiliter pertinet $H$. gratianopolitana Ramb. 1859 (sat frequens prope Grenoble Gallice). 
50. H. hispida Liv. (1758): t. solidula, modo depresso-conoidea, modo orbiculato-depressa, obsoletissime angulata, pilis brevibas curvatis. subpersistentibus hispida; apert. depressa, margine basali strictiusculo; diam. 8, alt. $5 \frac{1}{2} \mathrm{~mm}$. (Eur. bor. et media ad Alpes, Pyren. et Balkun.) T. sat late umbilicata, cinereo-cornea, sæepius cingula albida ornata, costulis transversalibus inæequalibus plerumque fasciculatis munita, anfr. $6-7$, lente accrescentes, rotundati, nltimus paullo ampliatus, sutura profunda, apertura obliqua, lunaris, inferne compressa, margine columellari perbrevi et basali substricto angulum perdistinctum, sæpe rectum, formantibus, labio candido paullo remoto, elato, in margine basali adhuc fortiore, extus conspicuo, munita. Fig. Rossm. Icon. fo 426, Jahrb.-1874. t. 12. fo 2. (Foss.) - Variat cornea, subrufa of fusca (numquam rufobrunnea.)

- var. nana JefFr. (1862): t. parva, depressa, umbilico latiore et labio validiore; diam. $52 / 5$, alt. $32 / 5 \mathrm{~mm}$. (Britan., German. media.)

- var. nebulata MKE (1828): t. minor, depressa, colore albido et labio fortiore; diam. $7 \frac{1}{2}$, alt. $4 \frac{1}{2} \mathrm{~mm}$. (Belgia, Gallica, German. boreal., Scandinavia.)

- var. conica JeFFr. (1862): to minor, spira elevata, anfr. $5-61 / 2$, arctioribus, ultimo vix latiore.

- var. depitata C. PFr. (1828): t. apertius umbilicata, subgloboso-depressa, nitida, omnino depilata, anfr. 6 ,angusti, convexi, apertura magis depressa, lunaris, intus albo-labiata, margine basali stricto cum columellari brevissimo præcipue angulum formante. (Germ., Scandin.)

- var. septemtrionalis CL. (1874): t. spira paullo convexiore, umbilico dimidium angustiore. (German. boreal., Scendin.)

- var. vendeana Letourn. (1869): t. depressa, vinosa aut rarius corneo, elegantissime striatula et pilis albidis brevissimisque hirsuta, anfractus ultimus vix major, apertura parum obliqua, lunata, transverse oblonga, peristomate acuto, recto; d. 10 , a. 5 mm. (Gall. prope Fontenay.)

- var. concinna Jerrr. (1833): t. late umbilicata, depressa, spira convexa, pallide cinerea vel pallide cornea, sæpe rúfo-brunneo strigata, pilis raris et caducis munita, striata, nitidula, anfr. 6-7, areti, apert. lunata, altitudine majori, peristomate intus albo-labiato; d. 8-9, a. $4^{1}{ }_{2}-5 \mathrm{~mm}$. (German., Britan., Dan., Suec.) 
- var. gyratus Westeru. (1871): t. depressissima, hispida, late umbilicata, ferruginea vel brunnea, spira plana, anfr. tardissime accrescentes, externi subsquales, apertura intus valide labiata; d. 10, a. $4 \mathrm{~mm}$. (Shec.)

- var. putoni Cless. (1874): t. anguste umbilicata, tenuis; anfr. 6-7 rotundati, celerius accrescentes quam H. hisp. $\alpha$, ultimus dilatatus; apert. late semilunaris, intus prope marginem albo-labiato; d. $84 / 5$, a. $5 \mathrm{~mm}$. (Belgia et in mont. Vogesis.) Fig. Cl. Exk. Fauna (1876) p. 114. Syn. H. glabella Put. 18t7, H. depilata Colb.

(H. umbrosa Partsch,)

B) Apertura labio destituta.

51. H. umbrosa Partscir ap. C. PFr. (1828): t. striatula, sub lente subtilissime et densissime granulata; apertura transverse ovalis; diam. 11-13, a. 6-6\% mm. (Austr., Carniol., Carinth., Styria, Tirol., Saxonia,-Bavar., Bohemia, Transsilv. - Dania?) T. late infundibuliforme umbilicata, valde depressa, raro convexa, obtuse angulata, tenuis, pellucida, cornea, albido lutescens vel virescens, anfr. 5-6, regulariter acerescentes, convexiusculi, ultimus ántice descendens, apertura sæe intus tennissime labiatum, peristomate expanso, marginibus approximatis. Fig. Rossm. Icon. f. 13 \& 421-425. (Foss.)

52. H. becasis RAmB. (1868): t. minima, irlegulariter striatoplicatula, tenuiter hispida; apertura rotundata, late lunata; diam. 4, alt. $21 / 4 \mathrm{~mm}$. (Gallia merid. infra mont. Conigou.) 'T'aperte umbilicata, depressa, spira convexa, subvirescens vel subrufescens, tenuis, pellucida, anfr. $4^{1} / 2$, convexi, ultimus citius accrescens, apert. peristomate simplici, recto, tenui, marginibus rotundatis subapproximatis. Fig. Journ. Conch. 1869. t. 9. f. 3.

**) Testa peranguste umbilicata vel perforata.

c) Apertura intus exacte calloso-iel denticulatolabiata. Testa conoideo-globosa.

53. H. edentula Dre. (1805): t. vix perforata, globoso-turbinata, basi planiuscula; anfr. $7-8$, ultimus obtuse subcarinatus; apert. angusta, depresso-lumata (subrectangularis), intus rufo- $\mathrm{r}$. albomarginata, edentula; diam. $5 \frac{1}{2}-6 \frac{1}{2}$; alt. $5-5 \mathrm{~mm}$. (Alpes in Savoyen, Wiurlemb., Bavas., Helv., Gall., Cavinth., Camiol. - In Holsatia, Penins. Cimbr. et ins. Füen Danic introducta?) T. cornea vel fulva, subopaca et hispidula, subtilissime striatula, anfr. convexiusculi, lentissime accrescentes, ultimus medio pellucide cingulatus, apert. marg. colum. subverticali perbrevi, basali subhorizontali 
et exter. brevi, labio extus a peristomate reflexiusculo tenui remoto, fulvescenti-pellucido. Syn. $H$. depilata Drp. 180 , $H$. liminiferce Held 1836. Fig. Rossm. Icon. f. 423. (Foss. perrara et minima: d. 5, a. $3 \frac{1}{2}$ $\mathrm{mm}$.) Variat depressior, perforatione niagis aperta. Obs. Num II. edentula Pfr. Mon. III. p. 125 e Carniolia et Bavaria merid. (t. conoidea, granuloso-striata, anfr. 6 convexi, apert. subtriangulari-lunaris, diam. 8 , alt. $6 \mathrm{~mm}$.) hue pertinet?

54. H. cobresiana ALT. (1812): t. perforata, globoso-conoidea, basi convexiuscula; anfr. $6-7$, ultimus rotundatus; apert. sat late lunaris v. oblique subtriangulari-lunata, intus rufo-vel albo-labiata, in medio margin. basal. unidentata; diam. 8, alt. 6 mm. (Europa media, Gall. austr.) T. cornea vel rufa, hispidula, subopaca. subtilissime striatula, anfr. convexi, lente accrescentes, sutura profundiusculit separati, ultimus medio albido-cingulatus, apert. marg. colum. et basali leviter curvatis, reflexiusculis, margine exteriore longo, arcuato, subrecto, labio fulvido v. rufescente pellucido, extus tenui, sæpe perlato, a peristomate tenui obscuro remoto. Syn. H. unidentata Drp. 1805, non Chemn., H. monodon Fér. 1821, H. ventricosa Jan. 1832. Fig. Rossm. Icon. f. $15 \& 432$. Variat minor, latius umbilicata (H. unidens Z.)

55. H. bielzi A. Scim. ap. Bz. (1868): t. obtecte perforata, depresse globoso-conoidea, subtus convexiuscula; anfr. $6-7$, ultimus rotundatus, obtuse angulatus; apert. late lunaris, intus exacte albo-Iabiata, peristoma subpatulum, rufum; diam. $6 \frac{1}{2}-10$, alt. 51/2-71/10 mm. (Transsitv., Bosna.) T. rufescens, pellucide cingulata, subtiliter striata et pilis brevibus hispida, anfr. convexiusculi, lente accrescentes, sutura tenuis, apert. obliqua, extus rufescens, margine columellari intus incrassato.

- var. bosnensis Möllend. (1873): t. major, depressior, diam. 11, alt. $7 \mathrm{~mm} \cdot($ Bosna.)

56. H. filicina Scmm. ap. Pfr. (2841): t. aperte perforata, depresso-globosa, subconoidea; anfr. 6, ultimus obtuse angulatus, antice subito descendens, apert. subovato-lunaris, intus roseo-labiata, margine columellari reflexiusculo; diam. 9, alt. $5 \mathrm{~mm}$. vel d. 8 , a. $6 \mathrm{~mm}$. (Carniolia.) T. rufescenti-cornea, albido-cingulata, substriata et pilis brevissimis sparsis obsita, anfr. convexiusculi, sutura tenui præ ceteris marginata disjuncti.

- var. nudata mh.: t. paullo major, depilata, umbilico paullo majore, labio obsoleto. (Bosna: Mlldf.)

57. H. loucozona Z. ap. C. PFr. (1828): t. plus minus late perforata, turbinato-subglobosa; anfr. 5-6, ultimus obtuse angulatus; apert. lunaris, subdepressa, margine basali substricto, in 
peristomate subpatulo, exacte albo-labiata; diam. $11-12$, alt. 7 mm. (Alpes Carinth., Camiol., prov. Austria litor., Ital. bor.) I. sæpissime anguste perforata, dilute rufescens, pellueide albo-cingulata, subtiliter striati, nitida, nuda, anfr. convexiusculi, lente accrescentes, sutura tenui et alba disjuncti, apert. extus fulvo-rufescens. Fig. Rossm. Icon. f. 436. Variat 1) albida, 2) t. anguste umbiticuta (II. dolopida Jan.)

- var. ovirensis Rossı. (1838): t. minor, obseurior, distinctius albo- fasciata, sxpe sub lente paullulum hirta, labio a peristomate paullo remoto. (Carinthia, mons Ovir, Ital., prov. Bergamo) Fig: Rossm. Icon. f. 434 .

- var. heteromorpha mh.: t. semiobtecte perforata, turbinata, striata, anfr. $7 ! / 2$, sat convexi; diam. 11, alt. $8 \% / 2 \mathrm{~mm}$. (Carinthia.)

3) Apertara intus sublabiate rel labio destituta.

a) Testa subglobosa iel globoso-depressa, ecarinata.

1) Testu muda, subnuda vel primo pilis raris et caducis hispida. (Anfr. ultimus antice plus minus (lescendens.)

-) Testa anguste umbilicatu.

58. H. liberta WesterL. (1870): t. subglobosa, tenuiter striatula; apertura rotundato-lunaris, albido-sublabiata; diam. 7-8, alt. 5-6 mm. (Suec. ad Lund, Baver. ad Dinkelscherben, Baden.) T. cornco-fusca vel rubescens, solidula, nitidula, pellucescens, pilis raxis calncis hirta, demum nuda, anfr. $5-5^{1} / 2$, regulariter accrescentes; convexi, teretes, ultimus paullo major, sulrotundatus, fascia albida eingulatus, sutura profundiuscula, antice sensim descendens, apertura margine columellari reflexiusculo. Syn.? H. hisp. var. subglobosa Jeffr. (Britun.), H. sericer Ald. et sape in coll. (teste Jeffr.), II. concima Dup. (Gall. in Jura). Fig. Jahrb. 1874. t. 13 f. 3.

59. H. pleboia DrP. $(1805)$ : t. globoso-depressa, irregulariter costulato-striata; apertura lunaris, tenue albido-labiata; diam. $11^{1} / 2$, alt. $7 \frac{1}{2} \mathrm{~mm}$. (Gallia.) T. corneo-lutescens, soliclula, pelluceseens, pilis rariuseulis et rigidulis hispida, demum nuda, anfr. 6, regulariter acerescentes, ultimus paullo major, vix obtuse angulatus, fascia albescente cingulatus, sutura sat profunda, ad aperturam paullulum descendens, apert. peristomate subpatulo, margine columellari reflexo. Fig. Iup. IIist. t. 8. f. 10; Jahrb. 1874. t. 13. f .5.

60. H. glabella Drp. (1805): t. subdepressa, subglabra, utrinque convexiuscula; apertura subovato-rotundata, lunata, sublabiata, marginibus alter ad alterum inflexis et subapproximatis; 
diam. 7-10, alt. 4-6 mm. (Gallia, German.) T. tenuis, anguste umbilicata, striatula, nitida, corneo-rufa vel rufo-virescens, rarissime et brevissime pilosa, anfr. $5-5 \frac{1}{2}$, convexiusculi, ultimus multo major, dilatatus et subtus ventriculosus, supra obtuse angulatus, non albidocingulatus, umbilicus angustus, pervirs, peristoma rectiusculum, noutum. Syn. H. telonensis Mittre 1842, H. lavandula Bourg. (teste Ramb.) Fig. Dup. Hist. t. 9. f. 1; Bourg. Moll. nouv. t. 8. f. 1-5.

- var. montoni Mrtтre ap. Dur. (1848): t. major, angustissime umbilicata, rufa, pallide albido-cingulata, apertura intus incrassatomarginata; d. $10-14$, a. $6-8 \mathrm{~mm}$. (Gall.) Fig. Dup. Hist. t. 9. f. 2 .

61. H. ætnæa BEn. (1857): t. subdepressa, hispida; apertura oblique lunato-rotundata; peristoma simplex, marginibus remotis; diam. $4 \frac{1}{2}$, alt. $2 \frac{1}{2}$ mm. (Sicilia, ad montem Actnct.) T. tenuis, anguste umbilicata, nitida, striatula, rufescens, anfr. $4^{1 / 2}$, convexiusculi, ultimus angulatus, non albido-cingulatus, peristoma simplex, acutum. Fig. Ben. Sicil. Illustr. t. 4. f. 12.

62. H. lanuginosa Bors. (1835): t. globulosa vel globosodepressa, hispida; apertura rotundata, late lunata, intus levissime callosa, rosea; diam. 14, alt. 9 mm. (Ins. Balear.) T. Iutescenticornea,-opaca, subrugoso-striatula, pilis brevibus densis hispida, apice glabro papillato, pilis detritis punctulis densissimis scabriuscula, anfr. $51 / 2$, convexiusculi, ultimus multo major, inflatus, sæpe obsolete albidocingulatus, superne obtusissime angulatus, peristoma subpatulum, acutum, margine columellari reflexo. Syn. H. roseotincta Forbes. Fig. Rossm. Icon. f. 571, 574.

63. H. corsica Shutru. (1852): t. depresse conico-globosa, tenuissime hispidula; apertura rotundato-lunaris, peristomate acuto, simplici, concolore; diam. 15, alt. 91/2 mm. (Corsicu, Sardinia.) T. anguste et semiobtecte perforata, striata, leviter angulata, tenuis, rufescenti-vel lutescenti-cornea, opaca, spira subconica, anfr. 5, sensim accrescentes, medio planiusenli, ad suturam mediocrem convexi, peristoma vix subpatulum, margine columellari breviter reflexo.

-) Testa vix perforata.

aa) Testa fugaciter pilosa et tenuissime labiato.

64. H. perlevis Shu're. (1852): t. major, subobtecte angustissime perforata, striatula, pilis minutissimis et fugacissimis sparse et vix conspicue induta: apert. rotundata, tenuissime labiata; diam. 14, alt. $11 \mathrm{~mm}$. (Corsica, Sardinie.) T. globosa, tenuissima, diaphana, pallide cornea, versus apicem erubescens, anfr. 6, sensim accrescentes, convexi, ultimus eximic rotundatus, apert. peristomate recto, acuto, margine columellari breviter dilatato-reflexo, 
65. H. psaturochæta BourG. (1860): t. vix perforata, tenue striatula et sub lente in adultis spec. paullulum irregulariter malleata, pilis densis brevibus caducisque hirta; apertura lunato-rotundata, peristomate simplici, acuto; diam. 9, alt. 6-7 mm. (Gall. ad ILorlaix.) T'. subglobulosa, fragillima, pellucida, pallide luteola, ad aperturam sæpius vinosa, anfr. 6, convexiuscula, regulariter acerescentes, peristomate in margine columellari subpatulo.

\section{(H. corsica Shuttl.)}

bb) Testa nuda, albolabiatu.

66. H. ovularis Bourg. (1855): t. vix perforata, elegantissime costulato-striatula, nuda; apertura rotuindata, albo-labiata; diam. 8, alt. $7 \mathrm{~mm}$. (Prope Adrianopol.) T. ventricoso-globosa, diaphana, nitida, cornea, anfr. $5 y_{2}-6$, convexi, regulariter accrescentes, sutura profunda separati, ultimus prope aperturam subito descendens, peristoma fuscum, simplex, acutum, in perforationem pallulum deflexum. Fig. Rev. Zool. 1855. t. 16. f. 7-9.

67. H. crenophila PFr. (1857): t. perforata, striatula, nuda; apertura ovato-rotundata, albo-labiata; diam. 7 , alt. $5 \mathrm{~mm}$. (Constantinop. et Caucasus.) T. globoso-depressa. nitida, cornea, anfr. 51/2: couvexi, regulariter accrescentes, ultimus lente prope aperturam descendens, peristoma fuscum, acutum, simplex, in perforationem deflexum. Syn. H. muscicola Bourg. 1855, non Phil. Fig. Rev. Zool. 1855. t. 16 . f. $10-12$.

\section{(H. pseudosericea Ben.)}

ff) Testa pilis mollibus densis sat persistentibus hirsuta, pilis detritis dense granulosa.

68. H. granulata ALD. (1830): t. perforata, subglobosa, spira acutiuscula, pilis minutis obtecta; anfr. regulariter accrescentes, ultimus major, infra planulatus, autice vix descendens; diam. 7-8, alt. 5-6 mm. (Britanniu.) T. pallidissime cornea vel pallide flavescens, anfr. 5-6, convexi, ultimus exacte rotundatus, non albido-cingulatus, apertura perobliqua, late lunaris, intus tenuissime albo-labiata, peristomate recto, acuto, margine columellari summo reflexo. Syn. Verosimiliter $H$. sericea Drp. (Gallia); H. globularis Jeffr. 1833. Fig. Jahrib. 187t. t. 12, f. 4.

- var. epirotica Mouss. (1859): t. perforata, pallide vel roseo-carnea, pellucida, tenuis, epidermide fugaci, punctis confertis minutis, nom pilosis, ornata. (Gracia in Janina.)

- var. mbiginosa (Z.?) A. Scris. (1853): t. sæpius minor, anguste umbilicata, corneo-fusca, v. rufescenti-brunnea, albide cingulata, pilis erectis distantibus hirta, anfr. ultimus supra mediam 
obtuse angulatus, apert. magis rotundata, margine basali fortius arcuato. (Suec., Dan., Hollandia, Germ. presertim bor., Transsilv., Rossia media of austr.?) Fig. Rossm. Icon. f. 428. - Animal sagitta unica prædita.

69. H. sericea (Drap.?, non MÜLL.!) Rosss. pr. p. (1838): t. perforata v. peranguste umbilicata, depresso-globosa, spira obtusa, pilis sat longis densis et bene persistentibus hirsuta; anf:. sat celeriter accrescentes, ultimus magnus, infra tumidulus, antice sensim descendens; diam. 7\%/2, alt. $5 \frac{1}{2} \mathrm{~mm}$. (Europa media et merid., montosa.) T. flavescenti-cornea, dense striatula, anfe. 6, convexiusculi, ultimus exacte rotundatus, demum dilatatus et ventriculosus, raro albido cingulatus, apertura late lunaris, in margine columellari leviter labiata, peristomate acuto, reflexiusculo. Fig. Rossm. Icon. f. 429; Jahrb. 1874. t. 12, f. 3. - Animal sagittis duabus prædita.

- var. cxpensa CL. (1874): t. magis depressa, lutescens; anfr. 5, rotundati, rapide accrescentes, ultimus dilatus; apertura magna, rotundata, non labiata; d. 7, a. $5 \mathrm{~mm}$. (Bavaria.) Fig. Jahrb. 1874. t. 12. f. 5.

- var. comeola CL. (1874): t. parva, globosa, lutescentivel fusculo-cornea, pilis brevissimis et tenuibus facile deciduis obsita; anfr. $5-5 \frac{1}{2}$, lente accrescentes; apert. subcompresso-rotundata, prope columellam callo debili munita; d. $5 \frac{1}{2}-6 \frac{1}{2}$, a. $4-$ $41 / 2$ mm. (Alpes Bavar. ct Helvet., Silesia.) Fig. Jahrb. 1874. t. 13. f. 2.

- var. dubia CL. (1874): t. magis depressa, anguste umbilicata, pallide cornea vel fuscescens, pilis brevibus facile deciduis obtecta, tum fortiter striata; anfr. 6, lentius accrescentes et ultimus minus dilatatus (quam in $\alpha$ ); apert. rotundata, semilumaris, labio prope columellam prominentiore munita; d. 8, a. $5 \frac{1}{2} \mathrm{~mm}$. (Tirolia ad Innsbruck.) Fig. Jahrb. 1874. t. 13. f. 1.

$\left(\frac{1}{6}\right)$ Teste squamulis densis, minutissimis, cinereis pruinosa.

70. $\mathrm{H}$. transsilvanica BıLz in sched. (teste Kob. 1871): t. anguste perforata, subglobosa, coriacea; apertura late lunata; diam. $5 \frac{1}{2}-61 / 2$, alt. $41 / 2-6 \mathrm{~mm}$. (Transsilv.) T. cornea, fusca aut virens, nitida, anfr. 5, planiusculi, ult. obsolete angulatus, interdum albido-cingulatus, peristoma vix patulum, acutum, membranaceum, ad umbilicum solum reflexum. Syn. $H$. sericea M. Bielz, H. fusca E. Bielz.

71. H. martorelli Bourg. (1870): t. profunde perforata, depressa, supra convexa, subtus subturgida; apertura transverse suboblongo-rotundata, lunata; diam. 6, alt. $3 \frac{1}{2}$ mm. (Hisp. prope Barcelona.) T. obscure cornea, fere semper limo inquinata, striatula 
ac undique pilis lamelliformibus hirsuta, anfr. $41 / 2-5$, regulariter crescentes, subangulati, supra leviter compressi, subtus sat rotundati, ultimo vix majore, sicut declivi ac lente descendente, peristoma șimplex, intus vix paullulum labiatum, margine basali leviter reflexiusculo, marginibus callo tenui junctis. Fig. Rev. Zool. 1870. t. 15. f. $12-16$.

b) Testa depressa, acute carinatu.

*) Testu perforutu.

72. H. ciliata VEN. ap. STUd. (1820): t. perforata, globosodepressa, squamulis membranaceis transversim dispositis aspera, carina membranaceo-ciliata; diam. 10, alt. $6 \mathrm{~mm}$. (Tirolia, Helv., Gullia, Sabuudiu.) T. corneo-fusea, aculeis squamulosis membranaceis in medio carine ciliata, anfr. 5-6, planiusculi, apertura ovatolunata, peristomate reflexiuseulo, roseo-labiato, intus vix incrassato. Syn. HI. hirsute Jan. 1832. Fig. Rossm. Icon, f. 430. Variat 1) major : t. diam. 12, alt. $8 \mathrm{~mm}$. , 2) minor: t. depilata, d. 8 , a. $5 \mathrm{~mm}$. (H. biformis $Z$.).

73. H. cinctella Drp. (1801): t. subperforata, subglobosodepressa, subtilissime striata; diam. 10-12, alt. $6-8 \mathrm{~mm}$. (GalTia merid., Austria prov. litor., Italia, Sicil.) T. lutescens vel rufescens, tenuissima, pellucida, ad carinam impellucide albido-cingulata, utrinque convexa, anfr. 5-6, planiuseuli, apertura ovato-lunata, ad carinan angulata, peristomate superne simplici, acuto, margine basali reflexiusculo. Fig. Rossm. Icon. f. 366.

**) Testa aperte umbilicata.

74. H. parlatoris 'BIV. (1839): t. depressa, supra plana, subtus convexa, pilis longis villosa; diam. 9, alt. $4 \frac{1}{3} \mathrm{~mm}$. (Sicilia.) T. cornea, carimata, anfr. 4, planiusculi, ultimus descendens, apertura subangulata, rotundato-lunaris, peristomate recto, simplici, acuto, marginibus approximatis. Fig. Rossm: Icon. f. 688.

75. H. reina BEN. (1857): t. lenticularis, convexiuscula, basi subinflata, pilis rariusculis ornata; diam. $61 / 2$, alt. $3 \mathrm{~mm}$. (Sicilia.) T. cornea, subdepressa, subearinata, subdiaphana, anfr. 4, planulati, ultimus vix deflexus, apertura diagonalis, subovata v. lunato-rotundala, vix angulata, peristomate simplici, acuto, marginibus conniventibus. Syn. H. reince "Aen." Pfr. 1856. Fig. Mal. B1. 1856. t. 3. f. 14-17; Ben. Ill. Sic. t. 4. i. 11.

8. Monacha Harta. Testa perforata, depresso-globosu, scepe minutissime granulata, alutacce; anfractus ult. antice plus minus deflexus v. descendens; apertura obliqua, oblongo-lunaris, peristomute acuto, reflexo, intus valide labiato. 
Habitat in memoribus, locis humidis et umbrosis, inter folia putrescentia, in fruticetis et sub herbis. Fossilis in str. miocenico infero et in str. sequentibus.

\section{$\uparrow$ Testa peristomate recto.}

76. H. hiberna BEN. (1859): t. flavida, pilis minutis brevissimis villosa, subtilissime striata; apertura lunato-rotundata; diam. 9, alt. $5 \frac{1}{2} \mathrm{~mm}$. (Sicilia.) T. obtuse perforata, subglobose-depressa, tenuis, anfr. 5, subplanulati, ultimus subangulatus, basi convexus, apert.-peristomate subpatulo, intus albido-labiato, margine columellari reflexo. Fig. Ben. Ill. Sicil. t. 3. f. 23.

77. H. consona Z. ap. Rossir. (1839): t. lutescenti-cornea, anfr. super. punctulis elevatis minutissimis pseudopiliferis obsita, fascia intermedia pellucida, leviter striata; apertura late lunaris; diam. $14^{1} / 2$, alt. $9^{1 / 2} \mathrm{~mm}$. (Sicilice, Ital. in Calabria, Gracia ad Athenas, teste Bg.) T.obtecte perforata, depresso-globosa, nitidula, pellucida, anfr. 51/2, convexiusculi, peristoma rectum, acutum, intus albo-labiatum, margine columellari dilatato. Fig. Rossm. Icon, f. 572, 573.

\section{ti Testa peristomate expanso. \\ *) Testa minutissime granulata vel punctale.}

78. H. incarnata MüLL. (1774): t. anguste umbilicata vel perforata, sub lente minutissime granulata et squamulis minutissimis pruinosa; apertura subovato-lunata, peristomate lato patulo v. reflexo; diam. 12-13, alt. $8+9 \mathrm{~mm}$. (Europa.) T. globosa vel sæpius depresso-globosa, obsolete angulata, subopaca, rufescens, solitla, striatula, pellucide cingulata, anfs. 5-6, vix convexiusculi, ultimo multo majore, al aperturam late fulvo. Syn. Junior $=I t$. sericen Müll. 177t. Fig. Rossm. Icon. f. 361. Variat 1) pallidula M. 'T. testa pallida, tenuis; 2) albida; 3) obtecta, t. umbilico fere prorsus obtecto; 4) minor: diam. 11, alt. $7^{1} \frac{1}{2} \mathrm{~mm}$; 5) major: d. 18, a. $12 \mathrm{~mm}$. (Gall.); 6) silvestris Hartm. t. umbilico subaperto, pervio.

- var. armate $\mathrm{S}_{\text {тАв. }}(1859)$ : t. margine columellari peristomatis tuberculum callosum ferente. (Ital. bor., Helv.) H. monodon Villa, nec Fér.

- var. velebitana Stentz: t. crassa, solida, peristomate perlate patulo, margine basali strictiusculo. (Dalm., Bosna.)

79. H. nicosiana Mouss. (1854): t. pervie umbilicata, granulata; apert. lunato-elliptica, tenue labiata, peristomate expansiusculo, margine columellari late reflexo; diam. 12, alt. $7 \mathrm{~mm}$. (Gallipoli.) T. globoso-depressa, tenuis, opaca, rufescens, pallide unifasciata, striata, granulis separatis sed confertis irregulariter et obli- 
que sertis ornata, anfr. $5 \frac{1}{2}$, planiusculi, ultimus vix subangulatus. Fig. Mlouss. Cor. Bell. t. 1. f. 2.

- var. pallide Mouss.: t. subdepressa, fragilis, subgranulata, apertura minor, lunato-rotundata, marg. colum. vix protracto.

80. H. vicina Rossir. (1842); t. obtecte subperforata, distincte granulato-alutacea, juvenis pruinosa, apertura rotundatolunaris, albo-labiata, peristomate anguste patulo; d. 13, a. $9 \mathrm{~mm}$. - d. 14, a. $10^{1 / 2}$ mm. (Transsilv. fere tota, IIontes Carpath., Siles., Bohem.) T. depresso-globosa v. sæpius globosa, spira elata, conica, tenuiuscula, corneo-lutescens, medio pellucide lencozona, in statu juvenili squamulis minutissimis pruinosa, striatula, anfr. 6 , convexiuseuli, lente accrescentes, ultimus rotundatus, major, sed multo minus dilatatus quam in $\mathrm{H}$. incarn., sutura sat profunda. Syn. $H$. carpatica Friv. ined., H. tecta Z. ap. A. Schm. 185t, H. obtocta Scholtz. Fig. Rossm. Icon. f. 689. (Foss.)

81. H. lurida Z. ap. Rossu. (1837):-t. subobtecte perforata, lirta (pilis detritis dense punctata); apertura late lunata, albidolabiata, peristomate reflexiusculo; diam. 13, alt. $8 \mathrm{~mm}$. (Curniolia, Gracia in monte St. Elie.) T. globoso-depressa, spira conicoacuta, obtuse angulata, cornea, albido-cingulata, striatula, anfr. 6, convexiusculi, ultimus major, utrinque leviter depressus. Fig. Rossm. Icon. f. 360 .

*) Testa nec granulata, nee punctuta.

82. H. redtenbacheri Z. ap. PFr. (1856): t. anguste umbilicata; anfractus ultimus rotundatus, antice deflexus; apertura lunato-circularis; diam. 14, alt. 81/2 mm. (Ins. Syru.) T. conoideodepressa, solida, conferte striata, nitida, pallide cornea, spira conoidea, acutiuscula, anfr. 6, convexiusculi, apertura perist. intus crasse albo-labiato, marginibus approximatis, dextro breviter expanso, columellari dilatato et basali reflexis. Fig. Mal. Bl. 1856. t. f. 7-11.

83. H. limbata DRP. (1805): t. subobtecte perforata, subcarinata, carina alba, opaca; anfr. ultimus antice sub carina descendens; apertura ovato-lunata; diam. 15, alt. $9 \mathrm{~mm}$. (Gallia merid.) T. depresso-globosa, utrinque convexa, quandoque supra subconica, subtilissime striatula, pellucide corneo-lutescens vel rubescens, anfr. 5-6, convexiusculi, ultimus multo major, apert, perist. reflexiusculo, intus leviter albo- vel fusco-labiato. Fig. Rossm. Icon. f. 362. (Foss.) Variat minor: d. 10, a. $7 \mathrm{~mm}$.

9. Carthusiana Ков. Testa perforata, globoso-depressa, spira conoideo-exsertu, acutiuscula, temis v. solidula, subopaca, coerulescens, lutescens vel corneo-albida; anfr. 
6, sat forte accrescentes, uttimis dilatatus, antice plus minus descondens, pone aperturam transversim fulvus; apertura magna, scope ovato-lunata, semper albo-vel rubello-labiata, peristomate acuto.

Habilat in fruticetis, hortis et nemoribus, locis humidis.

广 Testa anguste umbilicata vel perforata.

84. H. cantiana MIonT. (1803): t. umbilicata, depresse globosa; anfr. ultimus rotundatus, inflatus, antice lente descendens; apertura rotundato-lunata, albo-labiata, pallide marginata; diam. 20-21, alt. 12-13 mm. (Britan., Belgia, Gallia bor., Illyria, Germ. ad Eckwarden.) T. tenuis, pellucida, fragilis, striata, corneoIntescens, sericina, interdum aperturam versus rufescenti-brunnea, intus margaritacea, anfr. 6, convexiusculi, rapide accrescentes, ultimus multo major, inflatus, dilatatus, subrugosus, apert. peristomate testro concolori, marg. basali areviato, columellari dilatato. Syn. H. carthusiana Drp. 1801, 1805, H. pallide Jeffr., et Donovan (1803). Fig. Rossm. Icon. f. 36t. Variat 1) subglobosa, 2) solidulie; 3) minor: d. 15, a. 10 mm. \& 4) apertura subovata. (Cfr. H. ors, v. app.)

- var. cemenelea Risso (1826): t. anguste umbilicata, tenuissime regulariter striatula, apert. rotundato-subovata, albo-labiata, fusco-marginata. (Gall. merid., Ital.bor., Corsica.) Variat: d. 11/1/2 $-16^{1 / 2}$ a. $8 \frac{1}{2}-9 \frac{1}{2}$ mm. Syn. H. galloprovincialis Dup. 1848, nec Matheron 1842. Fig. Dup. Hist. t. 9, f. 5.

- var. mublla Risso (1826): t. solidula, ruguloso-malleata, subglobosa, antice rubescens; d. 19, a. $15 \mathrm{~mm}$, vel minor. (Gall. merid., Itul. bor.) syn. H. dacampi Villa.

85. H. carthusiana MüLL. (1774): t. anguste perforata, supra planulata, spira ad apicem exserta, infra convexa; anfr. ult. superne obtuse subángulata, antice deflexus; apertura ovato-lunata, rufescenti-labiata, fusco marginata; diam. 12, alt. $71 / 2 \mathrm{~mm}$. (Europa med., oce. et merid.) T. albida, lutescens v. lactescens, antice rufescens, nitidula, rarius fuscula (incola mortuo lacteo-opaca), vix supra subtilissime striatula, solịdula, intus pallide cornea, pellucescens, anfr. subplanulati, forte accrescentes, ultimus dense tenuissime subscrobieulatus, apert. depressiuscùla, peristoma fere reetum, marg. basali elongato, strictiusculo, colum. reflexo perforationem temitegente. Syn. H. cartusianella Drp. 1801, Zen. bimarginate Gray 1821, H. gibsi Leach 1833, H. olivieri Fér., Mich., C. Pfs. Fig. Rossm. Icon. f. 366. Variat 1) major: d. 18, a. $9 \mathrm{~mm}$. (Eur. merid.) et 2) fasciata: anfr, ult. superne et spira ad suturam fascia rufescente. 
- var. rufulabris Jefrr. (1830): t. globoso-depressa, polita, anfr. sat lente accrescentes, apert. rotundato-lunata, anguste sed alte rufescenti labiata, margine intus rufo-lorumneo; d. $8-10$, i. 6-8 mm. Variat minor: d. 7, it. $5 \mathrm{~mm}$.

- var. leucoloma STab. (1864): t. minor, peristomato omnino albo. (Ital. sup.)

- var. radiata mh: t. depressa, non planulata, porcellanea, anfractubus omnibus utrinque radiis transversis numerosis e labiis trinslucescentibus ornatis, apert. horizontalis, marg. basali substricto, peristomate omnino albo, labio lato niveo. (Ital. ad Viareggio: nobilissima Paullucci ex. misit.) Monstrositas?

- var. archimedea BEN. (1859): t. angustissime perforata, subglobosa, anfr. rotundati, ult. antice vix descendens, apert. ovata. rubello labiata; d. 13 , a. $10^{1 / 2} \mathrm{~mm}$. (Siciliru.)

86. H. frequens Mouss. (1859): t. anguste perforata, depresso-convexa; anfi. regulariti, ultimus rotundatus, vix descendens, apert. rotundato-lunaris, albo-labiata, peristomate recto, margini columellari subito breviter reflexo; diam. $7 \mathrm{~mm}$. (Turcia tota; Ross. merid.) T. striata, saturate cornea, nitida, anfr. ultimus unicolor, apert. intus pallide cornea, perforatio semitecta; testa omnino nuda (H. cant. et carth. anfr. superioribus sub lente punctulis elevatis, olim piliferis, obsitis); diff. preterea a cantiana magnitudine, spira elevatiore et conico, anfr. supra convexioribus, colore et perforatione. In Transcaucasia ross. occurrit var, obscarce Mrouss., major (d. 12, a. $3 \mathrm{~mm}$.), convexior, anfr. 7, convexis, labio tenui.

87. H. carascaloides Bourg. (1855): t. angustissime umbilicilta, convideo-depressa; anfr. ult. rotundato-lunaris, pone limbum albo-labiata, peristomate recto, margine columellari supra umbilicum perdilatato; diam. $181 / 2-20$, alt. $10 \mathrm{~mm}$. ('Turciu prope Gullipolim.) T. leviter et irregulariter ruguloso-malleata, diaphana, lutescens v. cornco-albida, anfr. 61/2-7, regulariter accrescentes, apert. intus submargaritacea. Fig. Bourg. Amèn. I. t. 13. f. 1-3.

88. H. lamalouensis REyN. (1870): t, minime perforata, subglobuso-convexa; anfr. ultimus rotundatus, antice descendens; apert. lunito-rotundata, albo-labiata, peristomate recto; diam. 9, alt. 6\%/2 mm. (Galliu dep. Heruult.). T. eleganter sub lente striatula, pallicle griseo-albidula, in ultimo fusco-luteola, ad aperturam castanca, anfi. $61 / 2$, lente crescentes, convexiusculi, ultimus vix major, apertura leviter obliqua.

89. H. pirajnea BEN. (1859): t. pervio-perforata, subglobosa: anfr. ultimus antice descendens; apert. subovata, allido-labiata, 
peristomate subrecto, marg. colum. reflexiusculo; diam. 18, alt. $14 \mathrm{~mm}$. (Sicilice in mont. Madonie.) T. tenuis, nitida, striato-rugosula, cornea-flavescens v. carnea, anfr. 6, convexi, celeriter accrescentes. Fig. Ill. Sic. t. 3. f. 21.

90. H. rissoana PFr. (1846); t. perforata, conoideo-globosa; anfr. ult. basi inflatus, obsolete angulatus. pallicle cingulatus, antice deflexus; apert. magna, lunato-rotundata, rubello-labiata, peristomate undique subrqualiter angulatim expanso; diam. 18-20, alt. $13 \mathrm{~mm}$. (Grecia.) T. tenuis, striata, nitida, rufa, anfr. 6, convexiusculi, apert. semicircularis, margine colum. supra perforationem dilatato. Rectius varictas $H$. schuberti Roтн, in Caria occurrentis speciei.

91. H. anconæ Iss. (1872): t. anguste perforata, subglobosa, sæepe albo-unifasciata; anfr. ultimus dilatatus, magnus, rotundatus, paullo descendens; apertura lunato-rotundata, labiata, margine colum. reflexo; diam. $12-14$; alt. $8 \frac{1}{2}-10 \mathrm{~mm}$. (Italia prov. Liguria, Toscana, Pisana, ins. Elba \&:c.) T. tenuis, fragilis, corneolutescens, irregulariter striatula, anfr. 5-6, convexiusculi, regulariter accrescentes, sutura valde impressa, peristoma acutum, vix reflexum, perforatio margine colum. fere obtecta. Syn. H. olivieri Iss. 1866.

92. H. pseudosericea Bew. (1859): t. pervie perforata, subgloboso-depressa, pilis brevissimis subinconspicuis subobsita; anfr. ultimus subangulatus; apertura late lunaris, rufulo-labiata, marg. colum. reflexiuseulo; diam. 10, alt. $6 \mathrm{~mm}$. (Sicilia.)- T. tenuis, nitidula, substriata, corneo-rufescens, anfr. convexiusculi. Fig. Ben. III. Sic. t. 3 f. 22 .

H. pisiformis PFr. 1846 (t. perforata, globulosa, corneo-lutescens, linea 1 alba opaca cingulata, anfr. penult. subcarinatus, aperte rotundato-lunaris, remote et valide albo-labiata, d. 11, a. $7 \mathrm{~mm}$.) in Grweia ad Phigalia-Bassa: Saulcy teste Bourg. Cat. rais. p. 25 non vera in Caucaso inventa species, sed $H$. lurida Z., affirmante clariss. E. v. Mart. in Mal. Bl. XX.

\section{t+ Testa imperforata vel rimata.}

93. H. olivieri FER. (1821): t. demum clauso-perforatx, albidolutescens, anfr. ultimo antice rufescenti-corneo, medio anguste albocingulato, superne fascia obscura sat lata, usque ad apicem porrecta, ornato; apert. lunato-rotunda; diam. 14-15, alt. $10-11$ m.m. (Sicilia, Ital. merid., Gracia, Dalm., Turcia.) T. subglobosa, sat tenera, pellucescens, nitidula, medio obtuse angulata, obsolete striata, infra interdum obsoletissime scrobiculata, anfr. 6, rotundati, convexiusculi, ultimus infra convexior, interdum fascia altera obscura infra angulum albo-cingulatum, apertura pone peristoma rectum, 
obscurum albolabiata, margine columellari reflexo, basali arcuato. Fig. Rossm. Icon. f. 365 .

- var. parumcincta PARR: t. minor, fortius striata et rugulosa, tota cornea, fascia albida ad suturam et angusta ad peripheriam.

- var. bicincte BEN. (1859): t. corneo-rufescens, fasciis duabus lacteis cincta, anfr. ult. vix descendens, apert. vix obliqua. (Sicil.) Fig. Ben. 11l. Sic. t. 3. f. 16.

- var. rothi PFr. (1841): t. opaca, conoidea, alba, bifasciata, fascia supera angusta, rufa, inferiori lata, diluta, anfr. ult. vix descendens, apertura lunaris; d. 13, a. $9 \mathrm{~mm}$. (Ins. Syrr.) Speries distincta?

- var. cribrata mh.: t. nitidissima, coerulea, anfractu ultimo vix angulato dilute brumneo-bifasciato, lineis distantibus elevatis numerosis a sutura ad regionem umbilicalem radiato, ubique itiam oculo nudo confertissime ruguloso-malleato, impressionibus oblongis longitudinaliter positis, infimis vero transversim in circulo denso; d. $13 \frac{1}{2}$ - a. $9 \mathrm{~mm}$. (Grecia ad Athenas.) Forma testre et

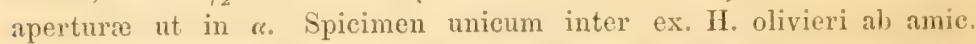
Kobelt recepta inveni, si plura requalia fuissent non dubitassem speciem optime distinctam in forma videre.

94. H. syriaca Enrens. (1831): t. subimperforata, grisea, fasciis 2 calcareis opacis ornata; apert. late lunaris; diam. 121/2, alt. $7 \frac{1}{2} \mathrm{~mm}$. (Sicilia.) T. globoso-depressa, parum nitida, minutissime rugulosa, spira convexiuscula, anfi. 5, planiusculi, peristoma subpatulum, frufum, albolabiatum, margine basali strictiuseulo. Syn. $H$. onichina Rossm. 1839. Fig. Rossm. Icon. f. 568.

- var. gregaria Z. ap. Rossm. (1839): t. corneo-lutescens vel rufula. unicolor, vix nitidula, striatula, anfr. 6 , convexiuseuli; d. 11-12, a. 7-8 mm. (Sicitia.) Fig. Rossm. Icon. f. 569. Ben. Ill. Sic. t. 3. f, 19.

95. H. cruzyi Boura. (1855): t. rimata, pallide albido-cornea, unicolor; apert. lunato-rotundata; diam. 7, alt. $5 \mathrm{~mm}$. (Sebastopol.) T. globoso-depressa, diaphana, elegantissime substriatula, anfr. 6, convexi, ultimus vix deflexus, subtus prope umbilicalem rimatoperforationem convexo-devians, apert. albo-labiata, peristomate arcuto, simplici, vix rubello. Fig. Bourg. Anén. I. t. 13. f. $4-6$.

H. æenensis BEN., Sicil. ins. Favagnana, et $H$. draxleri ZEL. ap. Kob. Cat., Græc. Arehipel, sunt mihi ignotre.

$$
\text { (II. consona } / . \text {.) }
$$


10. Eulota Hartar. Testa umbilicata, vavius perforata, inflata, subglobosa; anfr. 6--7, rotundati, ultimus subventricosus; apert. rotundato-lunata, sapissime labiate.

Habitat in silvis et fruticetis, præcipue humidis et prope æquas sitis. Fossilis in strato pleistocenico toto.

+ Testa unbiticata, unicolor (brunnea, variat hyalina) vel unirufofasciata, sulculis parallelis creberrimis undulosis longitudinalibus ornate.

96. H. fruticum MüLc. (1774): t. umbilicata, globoso-conoidea, basi inflata, albido-lutescens v, rufescens, raro fascia rufa aut castanea; diam. 18-20, alt. 14-15 mm. (Europa.) T. pertenue inæqualiter striata, anfr. 5-6, convexi, regulariter accrescentes, ultimus magnus, rotundatus, apertura lunato-rotundata, marginibus acutis, intuis roseo- aut violaceo-sublabiatis, columellari reflexo. Variat 1) major: d. 2t, a. $19 \mathrm{~mm} ., 3)$ minor: d. 19, a. $13 \mathrm{~mm}$. (v. turfica Slav.); 3) conoidea Westerl.: t. alba, acuto-conoidea, d. 19, a. $20 \mathrm{~mm}$. (Snec.); colore variat cinerea, rufula, fuscosa, rubella et alba, fasciata v. sæpius concolor. Syn. H. terrestris Gmel: 1788, H. carduelis Reib. 1855. Fig. Rossm. Icon. f. 8. (Foss.)

97. H. schrenki Midd. (1851): to angustius umbilicata, depresso-globosa, basi non inflata, diaphana, corneo-albida, rufounifasciata, diam. 15, alt. 9-11 mm. (Sibivia, Rossia media, Fennia merid.; Succ.bor?) Differt a præcedente testa minore, tenuiore, depressiore, colore suo normali apud fruticum perraro. Variat 1) major: d. $21^{1} / 2$, a. $\left.16 \mathrm{~mm} ., 2\right)$ minor: d. 13. a. $9 \mathrm{~mm}$. Fig. Middend. Sibir. Reise II. t. 30 f. 20 - 26 .

\section{ti Testa umbiticata, unicolor (albida) rel fasiis 2 albis cincta, striato-rugosa.}

98. H. martensiana Tiв. (1869): t. anguste umbilicata, semiglobosa, solida, striato-rugosula, subgranulata, corneo-crelea v. dilute fulvida; anfr. 6, convexi, ultimus vix descendens; apert. ovato-circularis; diam. 21, alt. $12^{1 / 2} \mathrm{~mm}$. (Italia in Aprutiis.) T. nitidula, spira subpyramidalis, anfr. lente accrescentes, ultimus magnus, supra planulatus, basi subinflatus, peristoma acutum, intus rufulolabiatum, marginibus conniventibus, subexpansis, basali et columellari reflexis. Fig. Bull. mal. ital. 1869. t. 3 f. $3-5$. - H. apennina Porro est me judice huc referenda.

99. H. orsinii Porro ap. Villa (1841): t. aperte umbilicata, depressa, solidiuscula, striato-rugosa, fulva v. albida, unicolor vel fasciis 2 albidis; anfr. $6 \frac{1}{2}$ planiusculi, ultimus antice, de- 
scendens; apert. subovata; diam. 18, alt. $10 \mathrm{~mm}$. (Itatia in Apruliis.) T. nitidula, spira paulhulum elevata, interdum subexplanata, ultimus utrinque planulatus, peristoma acutum, intus remote labiatum, marginibus approximatis, columellari reflexo. Fig. Bull. mal. ital. 1869. t. 3 f. $9-11$.

Iti Teste late umbilicata, gramulata rel pilosa.

100. H. strigella DRP. (1801): t. globoso-subdepressa, solidula, subpellucida, albido-cingulata, fugacissime pubescens; apertura lunato-rotundata, margine basali valde arcuato; diam. 13-14, alt. 9-10 mm. (Europa.) T. pallide cornea, fulva, rubescens, rel raro rufescenti-brunnea, interdum albicla, srepe solida, striatula, interilum forte striata, pilis minutissimis sparsis vel granulis microsenpicis obsita, anfr. 6, convexinsculi, regulariter accrescentes, ultimus multo major, rotundatus, supra planulatus, infra convexus, perraro fascia obsoleta secunda albida, al suturam, apert. intus albo- vel rufolabiata, marginilous subapproximatis, basali et columellari reflexiusculis. Syn. II. syluestris Alten 1812, H. alteand Grertn. 1813. Fig. Rossm. Icon. f. 9. - Variat 1) major: t. sape valde hirta, diam. 17//2, alt. 11 mm. (T'ranssilv.), 2) minor: t. tenera, pellucida, pallida (German.), 3) minima: d. $11^{1 / 2}$, a. $6^{1 / 3} \mathrm{~mm}$. (Græc., Ital., Austria), 4) subglobose, angustius umbilicata, diam. 13 , alt. $8 \frac{1}{2} \mathrm{~mm}$. vel diam. 14, alt. $10 \mathrm{~mm}$. (Carinth., Snec.), 5) solidula Brus.: t. dimidio minor, solidior, clare luteocornea, albido-cingulata (Croatia), 6) apennina 'Tib. (1869): t. depressior, solidior, magis striata, cornea (Ital. in Aprut.)

- var. colliniana Bourc. (1863): t. umbilico latiore, infundibuliformi, apertura minore, vix lunata, maxime albido-labiata, marginibus callo tenui albido junctis, anfractibus eleganter rotundatis, lentius et regularius accrescentibus, ultimo exacte rotundato; d. 15, a. $9 \mathrm{~mm}$. (Norregia.) Fig. Bourg. Noll. nouv. t. 7. f. 1-3.

101. H. parreyssi PFr. (1853): t. conoideo-semiglobosa, solidula, rufo-cornea, fasciis 2 latis albis cincta; apertura subtriangulari-lunaris, margine basali substricto, calloso; diam. 10, alt. $6 \mathrm{~mm}$. (Italia in Aprutiis.) T. pervio-umbilicata, granulato-striata, fascia una alha suturali, altera peripherica, anfr. $5 \frac{1}{2}$, convexi, lente accrescentes, ultimus rotundatus, antice breviter lescendens, basi distinctius granulatus, apertura diagonalis, albo-labiata, margine basali obsolete dentifero. "Affinis II. leucozonæ Zgl. var. aperte umbilicatæe" (Pfr.).

102. H. erjaveci Brus. (1870): t. depresso-globosa, tenuis, fragilis, semitransparens, subtilissime striato-granulata; apertura lunato-rotundata; marginibus approximatis; diam. 14, alt. $7 \mathrm{~mm}$. (Croutia, Bosna.) T. nitida, allida, anfr. $5 \frac{1}{2}-6$, convexiusculi, ultimus antice descendens, apertura candide labiatum, peristomate reflexi- 
useulo. "Species intermedia inter $H$. strigellam et umbrosam; differt a strigella statura minore, striis debilioribus, umbilico apertiore, testa fragili et semitransparente. $H$. umbrosa'statura subtili et granulatione similis, sed $F$. erjaveci est major, magis elata, non carinata, ejus umbilicus minus apertus, peristoma magis curvatum et expansum."

Iftit Testa anguste umbilicata, perforata, vel vix perforcita.

*) Testa tenera et fragilis, pallicte comea, glabra, anguste umbiticata rel perforata, diam. $16-20 \mathrm{~mm}$.

103. H. inchoata Mores. (1845): t. perforata, ad suturam rugulosa; anfractus ultimus vix descendens; apertura late lunaris, subtilissime labiata, marginibus distantibus; diam. 20, alt. $14 \mathrm{~mm}$. (Lusitania.) T. subglobosa, tenuis, striatula, corneo-lutescens v.corneo-fusca, anfr. $6^{1} / 2$, parum co:lvexi, lente accrescentes, ult. inflatus, medio rufo-cingulatus, peristoma rectum, tenue, marg. columellari reflexo. Fig. Chemn, Cab. Hel. t. 131. f. 18, 19.

104. H. brigantina Mengo (1867): t. anguste umbilicata, globoso-turbinata, subtiliter striata; anfractus ult. breviter descendens; apert. semilunaris, peristomate simplici. (Lusitan. pr. Braganza.) T. tenuis, cornco-lutea. (Mengo.)

105. H. fruticola KRrN. (1833): t. angustissime umbilicata, orbiculato-convexa, striata; anfractus ultimus antice valde deflexus; apert. rotundato-lunaris, anguste albo-labiata, marginibus subconniventibus; diam. 17, alt. $11 \mathrm{~mm}$. (Tauria.) T. tenuiuscula, albida, subglobosa, anfr. 7 , rotundati, peristoma rectum, subsimplex, margine colum. superne dilatato, reflexo. Fig. Chemn. Cab. Hel. t. 97. f. 17-19.

106. H. bourguignati PFr. (1856): t. anguste umbilicata, globoso-depressa, minutissime malleato-striata; anfractus ultimus vix descendens; apertura rotundato-lunaris, albo-labiata, convergentibus; diam. 19. alt. $11 \mathrm{~mm}$, (Tuuria.) T. tenuis, diaphana, carnea, anfr. 6, convexiusculi, regulariter accrescentes, ultimus rotundatus, peristoma marg, basali reflexiusculo, colum. dilatato, patulo.

**) Testa membranacea, (raro comea), olivacea vel viridula, scepius pilosa, perforata rel vix perforata, diam. $5-10 \mathrm{~mm}$.

107. H. revelata Mici. (1831): t. perforata, tenuis, depresse globosa, hispida, striata; ipertura lunato-circularis, peristomate simplici vel tenuissime labiato; diam. $7-8$, alt. $5-5 \frac{1}{2} \mathrm{~mm}$. (Gallia mer.occid., Lusit., Britrn.) T. diaphana, corneo-virescens, olivacea 
vel flavescens, paullulum ruguloso-striatula, pilis sat longis, rigidulis, albis, basi nodosis, irregulariter positis hirta, anfr. $4-4^{1} / 2$, convexi, forte accrescentes, sutura pirofunda canaliculata separati, ultimus ventricosus, subtus inflatus, antice valde descendens, apertura parum lunata, peristomate tencro, acuto, subrecto, intus margaritaceo vel pertenue albo-labiato, marginibus approximatis, columellari sat reflexo. Syn. H. ponentina Morel. 18t5, H. occidentalis Recl. 1846. (II. lisbonensis Pfr. 18t6.) Fig. Chemn. Cab. Hel. t. 3t. f. 5-8. Dup. Hist. t. 8. f. 9 .

- var. ptilota Boung. (1860): differt t. vix perforata, utrinque convexiuscula, pilis mimimis hispidula, anfr. 4, rapide accrescentibus, ultimo antice vix descendente, apertura majore (peristomate acuto, simplici); d. $5 \frac{1}{2}$, a. $4 \mathrm{~mm}$. (Gall., Vannes.) Species distincta?

108. H. montivaga mh: t. anguste umbilicata, solida, depresse globosa, pilis brevissimis subobsoletis raris obsita, striata; apertura ovato-lumaris, valide albo-labiata; diam. 7, alt. $5 \mathrm{~mm}$. (Lusitanic ad Cintra.) T. pellucescens, virenti-flava, rufo-fasciata, fasciæ anguste zona albida lata disjunctæ, anfr. convexi, regulariter accrescentes, ultimus antice rotundatus, ${ }^{9}$ ad aperturam deflexus. postice obtuse angulatus, apertura obliqua, peristomate patulo, marginibus conniventibus, basali et columellari reflexiusculis, intus circumcirca callo crasso et lato candido micante labiato. Syn. H.occidentalis Pfr. 18 s pr. p. "frequentissima prope Cintra; diam. maj. 9, alt. $5 \mathrm{~mm}$." Speciem descriptam inter specim. H. ponentinæ a clar. Morelet benigne communicata inveni.

109. H. fusca Movт. (1803): t. imperforata, tenuissima, depressa, superne rugoso-plicatula; apertura late lunaris, peristomate simplici; diam. 9-10, alt. 5-51/2 $\mathrm{mm}$. (Britan., Gall. merid.occid.) T. utrinque convexa, glabra, nitida, succinenta rel virescens, subtus lævigata, interdum angustissime perforata, anfi. 5 , convexiusculi, ultimus obsolete angulatus, peristoma simplex, acutum, margini colum. reflexiusculo. Fig. Dup. IIist. t. 7. f. 11.

11. Campylæa Вк. Testa aperte umbiticata, orticulatodepressa, spira parum elata rel plana; anfi. 1//2-6, planiusculi, ultimus antice srepe descendens; apertura lunato-orata vel rotumitata, peristoma labiatum, marginibus interdum contimis, columella sapissime basi reflexo, libero.

IIabitat locis lapidosis et rupestribus, in montium preruptis, in scopulis et muris, sub lapidibus et foliis putrescentibus, præcipue in Alpibus et montibus ceteris meridionalibus. Fossilis rarissime in str. pleistocenico supero. 


\section{A) Encampylca.}

1) Orientales: Testa magna, olivaceo-fuscescens, concexa, superne et infra densissime spiraliter lineata, scepius fasciis 3 fuscobrunneis; apertura marginibus distantibus.

110. H. pouzolzi Desh. (1831): to aperte umbilicata, orbiculato-convexiuscula; anfr. 6, planiusculi; apertura late lunaris, peristomate incrassato, marg. dextro expansiusculo, basali reflexo, colum. dilatato; diam. 47, alt. $24 \mathrm{~mm}$. (Dalm., Albania, Serbia, Bosna.) T. opaca, solida, striata, nitidiuscula, fulvo-olivacea, fuscotrifasciata, fasciis lateralibus dilatatis, anfr. ultimus rotundatus, antice breviter deflexus, peristoma albidum vel sordide carneum. Syn. $H$. varronis Cantr., dalmatina Parr., ragusana Fér., savignyana Ehrenb., trizona Reeve. Fig. Rossm. Icon. f. 215, 459. Variat 1) trifasciata Bz., 2) bifascia Brus., 3) unifasciata Pfr., 4) obscura Bz., obscure olivacea, fasciis obsoletis, 5) unicolor Pfr., lutescenti olivicea, efasciata (H. gravosaënsis Mühlf.)

-- var. minor, unicolor, spira parum elevata, anfractibus rotundatis; d. 31 , a. $17 \mathrm{~mm}$. Kob.-Rossm. Icon. f. 984.

- var. minor Rossm., minor, 'convexa, olivaceo-virescens, fasciis perobscuris; d. $29-39$, a. $16-22 \mathrm{~mm}$.

- var. montegrina Z.: d. 34 , a. $20 \mathrm{~mm}$. (Montenegro.) H. macarana Mühlf. Fig. Rossm. Icon. f. 4599.

- var. bosnensis Kor. (1871): t. minor, depressior, nitidula, fasciis tribus distinctis, subrqvalibus; d. $28-35$, a. $13-17 \mathrm{~mm}$. (Bosna, Serbia.) Fig. Kob.-Rossm. Icon. f. 985.

111. H. pancici MöLliend. ap. Ков. (1872): t. late umbilicata, globuloideo-depressa; anfr. $61 / 2$, rotundati, infra planati; apert. Iate lunata, magis depressa, margine dextro recto, basali angulato-subreflexo; diam. 30 , alt. $20 \mathrm{~mm}$. (Serbic.) T. striata, olivaceo-fuscescens, faseiis 3 fusco-brunneis, anfr. ultimus antice leviter descendens. Fig. Kob.-Rossm. Icon. f. 983.

112. H. serbica Möllend. ар. Ков. (1872): t. mediocriter umbilicata, globuloidea; anfr. 6 , rotundati, infra convexi; apert. late lunata, peristomate recto, marg. colum. paullo expanso; diam. 27, alt. $20 \mathrm{~mm}$. (Serbia.) T. striatula, olivaceo-fuscescens, fasciis 3 fusco-brunneis, anfr. lẹviter accrescentes, ultimus antice paullo descendens. Fig. Krob.-Rossm. Icon. f. 982.

113. F. banatica PArtcn ap. Rossu. (1836): t. aperte umbilicata, globoso-depressa, carinata, rufo unifasciata, carina albida; anfr. $51 / 2$, planiusculi; apertura late lunaris, peristomate albo-labi- 
ato; marginibus reflexis; diam. 30, alt. $15-17 \mathrm{~mm}$. (Bunatus, Hungarie, Transsilv.) T. opaca, olivaceo-fuscula r. rufa, supra carinam albidam brunneo-unifasciata, anfractus sat ncerescentes. Fig. Rossm. Icon. f. 457. Variat major: d. 31, a. $20 \mathrm{~mm}$. \& minor: d. 24, a. $13 \mathrm{~mm}$.

2) Phalevatr: Testa subopaca, luteola rel patlide cornea, maculis et striis fuscis sepissime plus minus variegata, vestigiis epidermidis plerumque ad basin, concexa, utrinque striis impressis spiralibus dense ornatn, medio sapius rufo-unifasciata; apertura marginibus distantibus, supero subrceto, basali reflexo.

114. H. phalerata Z. ap. Rossur. (1836): t. convexa, luteola, in vitta albida fusco-unifasciata; anfr. ultimus rotundatus; apertura sublabiata; diam. 20-23, alt. 13-14 mm. (Alpes Carinthice et Carniolia.) T. aperte umbilicata, fusculo variegata, lineis impressis spiralibus crebris etiam oculo nudo distinctis, transversim striatula vel rarius striata, anfr. 6 , sat lente accrescentes, convexiusculi, ultimus antice descendens, unifasciatus (fascia spiram non amplectens), sutura impressa separati, apert. rotundato-lunata. Fig. Rssm. Icon. f. 159

- var, chamaleon Parr. ap. Pfr. (1868): t. minor, convexo-depressa, oblique striata, supra albido et fusco variegata, infra circa umbilicum albida unicolor, ad peripheriam brunnea; anfr. 5 , vix convexiusculi, ultimus antice parum $\mathrm{r}$. vix descendens, fascia castanea peripherica in vitta lata albida ornatus, apertura ovato-rotundata, lunaris; d. $17-21$, a. 10-11 mm. (Carinth. prope Malborgeth.) Fig. Kob, in Rossm. Ic̉on. f. 991. - An huc $H$. cingulata mut. anconce GesTL. in Bull. malac. ital. 1868. p. 40. t. 3. f. 9-11 ("t. minor, fusco-trifasciata, supra planulata, inferne valde convexa, umbilico expanso, apertura subrotunda, anfr. 5 planulati") ex Alvornia in Italia referenda? (Pfr.)

115. H. hermesiana PINı (1874): t. subgloboso-depressa, cinereo-rosen, pallida, fuscorufescente- vel obsolete unifasciata, striatula; apertura intus fusea, albo-labiata; diam. 24-25, alt. 14-15 mm. (Italia in monte Presolana in pror. Bergamo.) T. aperte umbi. licata, striis spiralibus sulo lente distinctis, solida, rarius absque fascia, anfr. $6-7$, rotundato-convexiusculi, lente accrescentes, ultimus dilatatus, subinflatus, spira convexiuscula, circulum magnum formans, apert. obliqua, rotundato-lunaris, marg. basali reftexo. Fig. Kob,-Rossm. Icon. f. 1080. Var. Tigurica Kob. (1876): t. minor, fere orbiculata, anfractu ultimo minus dilatato, apertura rotundata; d. 20, a. $10 \mathrm{~mm}$. (Limone.) Fig. Kob.-Rossm. Icon. f. 1081. 
116. H. olympica Rоти (1855): t. globuloideo-depressa, albida, medio fascia angusta, brunnea; anfr. ultimus rotundatus; apertura late rotundata, peristomate simplici, marginibus approximatis; diam. 22, alt. $16 \mathrm{~mm}$. (MLacedonia in monte Olympo, Thessalia.) Testa umbilicata, striata, anfr. 5 , regulariter accrescentes, rotundati, ultimus ad aperturam descendens, apertura marginibus callo tenuissimo junctis, basali ad insertionem dilatato, umbilici partem tegente. Syn. H. thessalonica "Mouss." ap. Albers. Fig. Kob. in Rossm. Icon. f. 990.

117. H. alpina FaUre-Big. ap. FéR. (1821): t. convexa, obtuse carinate-angulata, luteola, striata, efasciata; apertura exacte albo-labiata; diam. 18-19, alt. 9-10 mm. (Alpes Gallice.) T. umbilicata, globoso-depressa, utrinque convexa, tenuiter striata, albida vel grisea, sæpe corneo irregulariter maculata, anfr. $5-5^{1} / 2$, convexiusculi, ultimus supra et infra carinam antice evanescentem subæqvaliter convexiusculus, ad aperturam superne tumidulus et deflexus, apertura subhorizontalis, latior quam longa, ovato-rotundata, parum lunata, peristomate subreflexiusculo. Fig. Rossm. Icon. f. 158.

- var. alpicola mh.: t. globoso-conoidea vel depresso-globosa, anfi. 6, convexi, rotundati, carina ubique obsoleta, interdum nulla, apertura subverticalis, subrotundo-lunaris; d. 15-17, a. 10-12 mm. (Gall., Grande-Chartreuse.) Ab H. carascalensi persimili differt testa magis globosa, majore, solidiore, umbilico largiore, apertura rotunda, labiata et margine basali reflexo. Fig. Dup. Hist. t. 8. f. 2.

118. H. glacialis Thomas ap. Fér. (1819): t. convexiscula, leviter carinato-angulata, lutescens, plicato-striata, rufo-unifasciata; apertura leviter albo-labiata; diam. 16, alt. $8 \mathrm{~mm}$. (Tirolia, Italia bor.) T. aperte umbilicata, depressa, supra strigis et maculis transversalibus pallide brunneis notata, infra circa umbilicum albida, unicolor, extus, præcipue pone aperturam, lineis numerosis angustissimis longitudinalibus fusculis, supra carinam albidam fascia angusta rufobrunnea, usque ad apicem spiræ producta, anfr. $5^{1 / 3}$, convexiusouli, ultimus antice descendens, apertura marginibus convergentibus. Fig. Rossm. Icon. f. 507. Variat minor: d. 12-13, a. 6-6 $6_{1 / 2} \mathrm{~mm}$.

3) Frigidce: Testa subopaca, fusca et stramineo-variegata, vel albida, levissime carinata, convexiuscula, solida, striata, subefasciata; apertura marginibus distantibus, exteriore subrecto, basali breve reflexo.

119. H. schmidti Z. ap. Rossur. (1836) t. mediocriter umbilicata, fusca, maculis et strigis albis vel stramineis variegata, rugoso-striata, intus fusco-rufescens; diam. $19-20$, alt. $10-11 \mathrm{~mm}$. 
(Cumiolia, Carinthia, Transsile.) T. solida, orbiculato-depressa, anfr. 51/2, convexiusculi, regulariter accrescentes, apert. rotundatolunata, obliqua, peristomate late albo-labiato, extus lutescente. Fig. liossm. Icon. f. 156. Variat major: t. anfr. ultimo rotundato, vix minime carinato; d. 24 , a. $12 \mathrm{~mm}$.

120. . f. frigida $J_{A N}(1832)$ : $t$. late umbilicatil, cinereoalbida, unicolor, striatula, intus flavida; diam. 21-22, alt. 10-11 mm. (Alpes, Itatia bor.) T. solida, depressa, anfr. 5-6, convexi, sat lente accrescentes, ultimus antice vix descendens, apertura rotundato-lunata, peristomate albo-labiato. Fig. Rossm. Icon. f. 157, 108: Variat major: d. 25-28, a. $11^{1 / 2}-12 \mathrm{~mm}$.

- vax. insubrica JAN (1839): t. minor, lactea, linea brumnea zonata, apertura semilunaris; d. 18 , a. $9 \mathrm{~mm}$.

121. H. carascalensis Fér. (1819): t. anguste umbilicata, albido-lutescens, unicolor vel corneo-variegata et subfasciolata, intus fuscula; diam. 12--13, alt. $7 \frac{1}{2} \mathrm{~mm}$. (Pyrencee.) T. solida, depressa vel rarius subgloboso-depressa, tenuiter et irregulariter striata, grisea vel Iutco-virescente maculata, quandoque carina perobtusa antice cvanida albo-fasciata, superne fascia perangusta obseura terminata, anfractus 5-6, planulati-vel convexiusculi, apertura rotundato-lunaris, peristomate subsimplici, intus vix labiato. Fig. Russm. Icon. f. 570. Variat major: d. 16, a. $10 \mathrm{~mm}$.

122. H. velascoi Hid. (1867): t. anguste umbilicata, opacoalbida, striato, fulvo-corneo irregulariter et radiatim strigata; diam. 18, alt. $10 \mathrm{~mm}$. (Hisp. bor.) T. orbiculato-depressa, solidula, spira convexa, anfr. $5^{1 / 2}$, convexi, superi leniter accrescentes, ultimus rotundatus, dilatatus, parum descendens, apertura magna, rotundatolunaris, peristoma album, simplex. Fig. Kob-Rossm. Icon. f. 1097. - Variat minor, t. fusea, albostrigata, indistincte ad peripheriam albofasciata, umbilico angustiore; d. 14-15, a. 8-9 mm. (Pyrenæe, Pic du Midi.) Kob.-Rossm. f. 1096.

\section{(H. styriaca Frfld.)}

4) Faustinc: Testa umbilicata, supra cornea vel lutea, subtus fuscescens, vel unicolor fusca, subgloboso-depressa, mfo-unizonata, strialuta; apertura marginibus undique reflexis et subapproximatis, basali strictinsculo.

123. H. cornea Drp. (1S01): t. umbilicata, solida; apertura ovato-rotundata, lunaris, peristomate labiato, angulatim reflexo, marginibus valde approximatis; diam. 13-16. alt. $7-8 \mathrm{~mm}$. (Gallia mer., Italia bor.) T. nitidula vel subopaca, depressa, convexiuscula, striatula, superne pallide-comea, dilute unizonata, subtus 
et fascia castaneo-rufa, 'anfr. 5, convexiusculi, ultimus antice subito deflexus, apertura perobliqua, peristnmate late reflexo, acuto, carneo, margine basali substricto. Fig. Rossm. Icon. f. 96. Variat 1) obscure comea, 2) major: d. 17, a. $9 \mathrm{~mm}$. et 3) minor: d. 12, a. $6 \mathrm{~mm}$.

- var. castanea Rossir. (1836): t. castanea, unicolor. (II. squamatina Fér. Mus.) Fig. Rossm. Icon. f. 219.

124. H. faustina $Z$. ap. Rossm. (1836): t. late umbilicata, solidula, nitida; apertura lunato-rotundata, peristomate albo-labiato, angulatim reflexo, marginibus subconvergentibus; diam. 1821, alt. 9-10 mm. (Galizia, Transsilv., Silesia, Mroravia, Bohcmia.) T. subgloboso-depressa vel complanata, lævigata, supra lutea, rufo-unifasciata, subtus fuscescens, anfr. 5, convexi, ultimus antice paullo descendens, apertura ovato-rotundata, lunulata, margine basali arcuato. Fig. Rossm. Icon. f 93.

- var. satica Z. ap. PFr. (1848): 't. pavula, solida, aurantio-castanea; d. 15 , a. $8 \mathrm{~mm}$.

- Vir. cilrinuln Z. ap. PFr. (1859): t. minor, convexior, lutescens, unicolor, umbilico angusto; d. 14, a. $71 / 2 \mathrm{~mm}$.

- var. charpentieri Scroltz (1843): t. convexior, utrinque fusco luteobrunnea, fascia latiuscula, umbilico angusto; d. 18, a. $\mathrm{S}^{1} / 2 \mathrm{~mm}$.

- var. fortunata PARr. ap. Brelz (1867): t. minor, depressa, solidula, nitiduli, utrinque albo-lutea, anguste zonata, fasciis transversis obscuris, peristomate vix reflexiusculo; d. 14, a. $7 \mathrm{~mm}$.

- var. associata Z. ap. Rossu. (1867); t. major, depressiuscula, supra pallide straminea, subtus luteo-brunnea, zona mediana latiuscula, subtus linea pallida terminata; d. 21 , a. $10 \mathrm{~mm}$. Fig. Rossm. Icon. f. 374.

125. H. möllendorffi Ков. (1871): t. umbilicata, tenuis, opaca, pilis longis sparsis ornata; apertura ampla, late lunaris, subsecuriformis, peristomate albo-labiato, reflexo, marginibus conniventibus; diam. 14-18, alt. $10 \mathrm{~mm}$. (Bosna et Sertia.) T. subgloboso-depressa, striata, corneo-fusca, fusco-unifasciata, anfr. $5 \frac{1}{2}$, convexiusculi, ultimus antice subito descendens, marginibus distautibus, columellari superne dilatato, umbilici tertiam partem tegente. Syn. H. pancici Bielz 1869 (teste Möllend.) Fịg. Kob.-Rossm. Icon. f. 1094, 95.

126. H. rossmässleri Prr. (1842): t. perforata, tenera, nitida; apertura rotundato-lunaris, peristomate reflexo, tenui, acuto, absque labio, marginibus distantibus; diam. 12, alt. $6 \mathrm{~mm}$. (MIont., Carpath.) T. globoso-depressa, striatula, supra lutea, rufo-unifasciata, 
subtus fuscescens, anfr. $t^{1 / 2}$, convexiusculi. Syn. $H$. advena Rossm. (1812). Fig. Rossm. Icon. f. 687.

5) Pellite: Testa anguste umbilicata vel perforata, plus minus globose, costulata, striatu, fascia alba utrinque rufomarginata; apertura subcircularis, "lbo-labiata, marginibus scepe callo junctis (anfi. ult. antice prorsus deflexus).

127. H. pellita Fér. (1819): t. perforata, fulvida, intus malgaritacea, fascia alba, utrinque rufo-marginata, pilis erectis rigidis obsita; anfr. convexiusculi; apertura subcircularis; diam. 15, alt. $9 \mathrm{~mm}$. (Grecia.) T. orbiculato-convexa, costulato-striata, peristoma vix expansiusculum. Fig. Kob.-Rossm. Icon. f. 1090. Variat 1) minor: d. 13, a. $7 \frac{1 / 2}{1 / 2 m}$; 2) liveglingeri Zel. ap. Kob. (1876): t. parum major, anfractibus rotundatis, spira magis elata; d. 15 , a. $10 \mathrm{~mm}$. (Syra.) Fig. Kob. Rossm. f. 1092.

128. H. graphicotora BourG. (1855): t. anguste umbilicata, argute et confertim oblique sculpturata ac elegantissime minutim decussata, albido-fusca, cingulo albo vineo-marginato ornata; apert. lunato-circularis; diam. 15, alt. $10 \mathrm{~mm}$. (Gjecia, ins. Mritos.) 'T. depresso-globosa, peristomate vix reflexiusculo. Fig. Bourg. Amén. I. t. 16. f. 5-8. (Bg.) H. pellita var, in Kob.-Rossm. Icon. IV. p. 42, f. 1091 .

129. H. lecta FÉr. (1819): t. anguste umbilicata, alba, unicolor vel fasciis 3 angustis fuscis cingulata; anfractus ultimus basi ventrosus; apertura lunato-circularis; diam. 17, alt. $11 \mathrm{~mm}$. (Canclia.) T. globoso-dejressa, tenuis, regulariter costulato-plicata, anfr. 5, planiusculi, apertura perobliqua, perist. undique breviter expansum. Fig. Kob.-Rossm. Icon. f. 1093.

130. H. naxiana FÉr. (1819): t. anguste umbilicata, fuscu, albido-strigata et cingulo albo rufomarginato ornata; anfir. ult. subconstrictus; apert. lumato-circularis; diam. 14, alt. $10 \mathrm{~mm}$. (Ins. Naxos et Candia.) T. depresso-globosa, striata, anfr. 5, planiusculi, peristoma reflexum. Variat alba, cingulis 3 rufis, dian. 18, alt. $12 \mathrm{~mm}$. (Candia prope Suda.) Fig. Chemn. Cab. Hel. t. 79. f. 24, 25.

131. H. noverca Friw. ap. Prr. (1853): t. aperte umbilicata, fulvida, medio albo-fasciata, superue fascia angusta rufia; anfr. planiusculi; apertura parvula, lunato-rotundata, transverse subdilatata; diam. 14, alt. $8 \mathrm{~mm}$. (Candio.) T. conoideo-depressa, nuda (?), ruguloso-striata, minutim decussata, diaphana, sutura albomarginata, apertura'latior quan alta, margine'supero expanso, basali reflexo. Fig. Kob-Rossm. Icon. f. 1089. 
6) Cyclolabres: Testa umbilicata, pullide cornea (virescens, Tutescens vel coerulescens), depressa, subtiliter striatu; apertura perobliqua, marginibus continuis (anfr.ult. antice prorsus deflexus.)

132. H. cyclolabris Desir. ap. FÉR. (1821): t. aperte umbilicata et raripilosa, pallide cornea vel albo-lutescens, rufo-unifusciata; apertura ovato-rotundata, leviter albo-labiata; diam. 22 -23, alt. $10 \mathrm{~mm}$. (Grcecia.) T. depressa, spira convexa, fascia utrinque pallide marginata, anfr. 5, convexiusculi, ultimus rotundatus infra convexus, peristoma continuum, interdum solutum, margine supero expauso, basali reflexo. Fig. Kob.-Roäsm. Icon. f. 1055, 1088. Variat major: d. 25, a. $12 \mathrm{~mm}$. (Desh.)

- var. hymetti Mouss.: t. major, fusco 3 fasciata, vix pilosa, anfractu ultimo transverse magis dilatato, apertura ampliore, peristomate soluto, labio albo nitido; d. 27, a. $13 \mathrm{~mm}$. (Hymettus et Parnassus.) Fig. Kob.-Rossm. f. 1083, 8t.

- var. arcadia PARr. ap. FrFLd (1867): t. inflata, colore saturatiore, fusco-unifasciata, vix pallido marginata. (Tinos.) Fig. Kob-Rossm. f. 1086.

- var. hcldreichi SHutru.: t. striatula, dentissime et minutissime granulata, pilosa, albo-lutescens, fusco-unifasciata, spira subplana, anfi. $4 \frac{1}{2}$, planulati, apert. subcircularis, marg. basali latius supra umbilicum reflexo; d. 15, a. $81 / 2$ mm. Syn. H. cyclolabris Pfr. 1818. Fig. Kob.-Rossm. Icon, f. 1087. Variat major.

133. H. sphæriostoma BourG. (1857): t. pervio-umbilicata, compresso-depressa, striatula, diaphana, albo-lutescens, bi- vel trifisciata; anfr. $5 \frac{1}{2}$, planulati, apertura subcircularis, peristomate valde saluto, undique late expanso; d. 20-22, a. $10 \mathrm{~mm}$. (Thessalia.) Fig. Rev. Zool. 1857. t. 8. f. 1-3. Kob.-Rossm. Icon. f. $108 t$.

134. H. desmoulinsi FARIN. (1834): t. pervie umbilicata, subtiliter striata, corneo-virescens, subefasciata; apertura perdepressa, transverse ovata, marg. supero intus labiato; diam. 16, alt. $7 \mathrm{~mm}$. (Pyren. orient.) T. fere complanata, fragilis, hand nitidula, ad aperturam interdum zonula rubella vel tribus evaneseentibus ornata, anfr. 6, planulati, ultimus subcarinatus, apertura marginibus reflexis, lamina sublibera junctis. Syn. $H$, moulinsii Pot. \& Mich. 1838. H. cornea var. Rossm. f. 511,; M. T. Fig. Dup. Hist. t. 6. f.6. Variat minor: d. 12, a. $5-\mathrm{mm}$.

135. H. coerulans Müıle, ap. C. Prr. (1828): t. angùste ad semiobtecte umbilicata, irregulariter rugosi, coerulescenti- 
livida, intus fuscescens, nuda et efasciata; apertuxa subcircularis, peristomate simplici, marg. columellari labiato, subreflexo; diam. 17-18, alt. 8-9 mm. (Croatia, Dalmatia.) 'T. convexiuscula, solida, opaca, anfi. 5, convexinsculi, ultimus dilatatns, ilepressiusculus, Sym. 11. lacticina Z. ap. Rossm. 1837. Variat 1) hyllica Brus., major, elevatiuscula, striato-rugosa;2) depressa Zel., media, depressa, lavis, diam. ex. max. 16/1/2, alt. $7^{1 / 2} \mathrm{~mm}$. (Rossm. f. 375); 3) rugata Kutsch. \& Sandri, media, elevata, rugosa, unifasciata (Rossm. f. 607-608); 4) bulavicana Brus., media, depressa, lævis, subunifasciata, brunnescens; 5) zrmanjce lirus., minor, depressa, lævis, subunifasciata, diam. 15, alt. $81 / 2 \mathrm{~mm}$.

7) Tigrina: Testa late umbilicata, albido- et comeo-variegala, depressa, anfractu ultimo depresso, obtuse carinato, antice subito deflexo; apertura marginibus valde approximatis, reflexis.

*) Testa supva subtiliter migoso-striata, efasciata vel zona inframediana.

136. H. tigrina $J_{A N} \cdot(1832)$ : to depressa, spira subplina, transversim striata; anfr. ultimus superne carinatus, supra subplanus, infra convexus, pone carinam brunneo-fasciatus; columella elongata, subverticalis; diam. 22-23, alt. $9^{1 / 2}-10 \mathrm{~mm}$. (Lombardia.) T. sericina, tenuis, cornea, albomaculata, subtus pallicla, apice prominula, raro spira convexiuscula, anfr. 5, subplani, sutura carinata, apert. subcircularis, marginibus conniventibus, basali reflexo, sublabiato, albo. Fig. Rossm. Icon. f. 226.

137. H. fontenillii Micr. (1829): t. depressa, spira subconvexa, ruguloso-striatula, spiraliter lineata: anfr. ultimus medio perobtuse angulatus, utrinque subæqualiter convexiusculus. efasciatus; columella brevissima; diam. 18-21, alt. $8-10 \mathrm{~mm}$. (Gallia.) T. tenuis, albido corneoque marmorata, subtus pallida, anfr. 51/2 -6 , convexiusculi, sutura profundiuscula, ecarinata, apertura subovatolunaris, marginibus distantibus, peristomate reflexiusculo, intus albo marginato, acuto. Syn. H. tigrina var. michaudiana Rossm. 1838. Fig. Rossm. Icon. f. 510.

138. H. cantabrica IIn. (1873): t. depressa, spiria plaua, minutissime ruguloso-striatula, sub lente siepe punctis minutissimis impressis, in junioribus pilis brevissimis instructis; anf. ultimus medio obtuse angulatus, efaseiatus; columella elongata; diam. 13 alt. $4^{1 / 2} \mathrm{~mm}$. (IIspp. in Asturia.) T'. tenuis, corneo-pollucida, albide strigata et punctata, anfr. 5, convexiusculi, ultimus jenultimo fere duplo latior, antice subdilatatus, infra convexior, apertura lunato- 
circularis, peristomate recto, acuto, albido-labiato, marginibus approximatis. Variat paullo major, minus rugulosa et impresso-punctata.
**) Testc utvinque aqualiter arcuatim elcvato-costata, $f a-$ sciis usoletis.

139. H. gobanzi Fris (1867): t. depressi, sölida, costis albis; apertura perobliqua, lunato-subcircularis, intus carnea; dian. 21-23, alt. $10-11 \mathrm{~mm}$. (Tirotia merid.) T. albida, fasciis nonnullis fuscis, sæpe obsoletis, notata, spira breviter conoideoconvexa, sutura profundiuscnla, anfr. $5^{1 / 2}$, convexiusculi, ultimus depressus, obsolete angulatus, peristoma tenuiter labiatum, acutum, marginibus approximatis, callo tenuissimo junctis, basali reflexo, columellari dilatato, subverticali. Fig. Kob.-Rossm. Icon. f. 1078, 79. Variat. major: d. 27, a. $14 \mathrm{~mm}$. (Cfr. H. colubrinam, quæ proxima, teste amic. Kobelt.)

\section{**) Testu supra costulato-plicata, infra sublcovis, in- terrupte subtrifasciata.}

140. H. ziegleri Scmm. ap. Rossu. (1836): t. depressa, subplana, obtuse subcarinata; apertura perobliqua, subcircularis; diam. 20, alt. $7 \mathrm{~mm}$. (Carniolia.) T. albido-cæsia, anfr. 5, planiusculi, sutura sat profunda, peristoma labiatum, marginibus fere connexis, basali strictiusculo, tuberculifero, reflexo. Fig. Rossm. Icon. f. 154. Variat minor: t. fortius costulata, fasciis minus distinctis, d. 15-15, a. $5-6 \mathrm{~mm}$.

8) Zonat:a: Testa umbiticata, albidu, irregulariter striata, depressiuscula, scpissime trifasciata; apertura marginibus approximatis (anfr. ult. antice prorsus deflexus.)

*) Testa non spiratiter lineata, peristoma reflexum.

141. H. trizona Z. ap. Rossu. (1835): t. convexiuscula, lutescenti-albida; apertura rotundato-ovalis; diam. 30-32, min. 26, alt. 12-13 mm. (Banatus, Transsilv., Serbia.) T. castaneo-trizonata, striata, anfr. 5-5 $1 / 2$, subplanulati, peristoma"subsimplex. Fig. Rossm. Icon. f. 87.

- var. inflata E. A. Bz.: t. convexior, umbilico angustiore, margine columellari subsemitecto, (anfi. penultimo non visibili), apertura rotundiore, margine basali valde arcuato, anfractibus magis tumidis. (Sertia.) Fig. Kob.-Rossm. Icon. f. 997, 998.

- var. rumelica Z.: t. minor, aretius spirata; diam. maj. 22, min. 19. alt. $11 \mathrm{~mm}$. (Rumctia.)

- var. baleanica Frrw.: t. convexa, umbilico angusto, flavido-virescens, magis orbiculata, fasciis lateralibus dilutis, inferis 
hine inde confluentibus, peristoma tenue, late reflexum, albolabiatum; d. maj. 28, min. 25, alt. 13-14 mm. (Serbia, Croatia.) Fig. Kob.-Rossm. Icon, f. 999.

- var. frauenfeldi ZEs. ap. Prr. (1853): t. lactea, vestigiis epidermidis lutescentis vestita, striatula, fascia pellucida paI'tum perspicui ornata; anfr. ultimus dilatatus, apertura rotundatoovatil, perobliqua, margine supero subrecto; d. maj. 261/2, min. 2:1/2, alt. $13 \mathrm{~mm}$. (Serbia ad Meidampeck, Banutus ad Mehadia et in montib. adjac. transsilv.) Clar. Pfeiffer dicit testam fasciis tribus pellucescentibus ornatam esse. Fig. Kob.-Rossm. Icon. f. 1001 .

*) Testa non spiraliter lineata, peristoma superne rectum.

142. H. phocæa Rотн (1856): t. irregulariter striata, albidocresia, superne fasciis 1 vel 3 fuscis ornata; spira vix elevata; anfr. convexiusculi, ultimus antice dilatatus; apertura ovato-rotundata, peristomate vix labiato; diam. maj. 20, min. 17, alt. $9 \mathrm{~mm}$. (Giacia, mons P(rnassus.) T. depressa, subovata, anfr. leviter accrescentes, apertura subhorizontalis, margine supero crassiusculo, hasali leviter reflexo. Fig. Kob.-Rossm. Icon. f. 995. Variat 1) major, distincte trifasciata; d. maj. 25, min. $20^{1} / 2$, a. $10 \mathrm{~mm}$. (H. ornata Parr. in sched.), 2) minor, fasciis obsoletis; d. maj. 18, min. $15 \mathrm{~mm}$. (H. ploc. var. inornata Kob, in Rossm. Icon. f. 994.)

143. H. langi PArr. ap. Rossm. (1857): t. subtiliter regulariter striata, albida, fasciis tribus brunneis angustis ornata; spira convexiuscula; anfr. depressiusculi, ultimus antice non dilatatus, apertura ovali-rotundata, peristomate simplici; diam. 25, alt. 13 mm. (Gracia, mons Parnassus.) T. depresse suborbiculata, anfr. lente accrescentes, apertura perobliqua, margine exteriore deflexo, columellari breviter reflexo. Fig. Kob.-Rossm. Icon. f. 993.

141. H. gasparinæ Charp. ap. Кі́ов-Rossm. (1876): t. striata, coeruleo-albida, fascia fusco-brumea mediana in cingulo albo ornata; spira parum elevata; anfr. rotundati; apertura late roturdata, peristomate simplici; diam. maj. 23, min. 21, alt. $13 \mathrm{~mm}$. (Gracia, mons Olympus.) T. umbilicata, orbiculato-depressa, anfr. regulariter accrescentes, ultimus vix descendens, apert. parum obliqua, murginibus callo tenuissimo junctis, basali perparum reflexo, ad insertionem parum dilatato. Fig. Kob.-Rossm. Icon. f. 989.

\section{***) Testa spiraliter dense striata.}

19. H. intermedia FÉr. (1819): t. aperte umbilicata. perdistincte spiraliter lineata, rufo-trifasciata, fasciis lateralibus inter- 
ruptis; diam. maj. 16-17, min. 14, alt. 6-7 mm. (Illyvia, Croatia.) T. ruguloso-striata, lutescenti-albida, anfr. $5-51 / 2$, planiuseuli, apertura perobliqua, ovato-rotundata, marginibus fere contiguis, externo expanso, basali reflexo. Fig. Rossm. Icon, f. 155: "Variat 1) $m i$ nor: d. 13-14, a. 5-6 mm.; 2) efasciata.

- var. tetrazona JAN (1832) t. majuscula, tetrazona, umbilico paullo angustiore; d. 23 , a. $9 \mathrm{~mm}$. (Lombavdia.) Fig. Rossm. Icon. f. 508.

146. H. æmula Rossir. ap. Kstr. (1852) t. anguste umbilicata, sub lente tenuissime spiraliter lineata, fasciis angustis rufis contiguis ornata; diam. maj. 11-13, min. 10, alt. 5-6 mm. (Istria.) T. striata, opaca, supra albida, infra fusculo, lutescens, striata, anfr. 5, subplani, ultimus infra convexus, apertura lunato-circularis, intus concolor, marginibus conniventibus, supero vix expansiusculo, basali reflexo. Syn. $H$. ambrosi Strob. $1852, H$. martinatiana Betta 1852. Fig. Chemn. Cab. Hel, t. 121. f. 4-6.

9) Umbilicares: Testa latius umbiticata, olivacea vel comeo-Hlavescens, scepius nitida et solida, glabra (rarissime pitis nommullis), striatula, supra medium scpissime in albida ritta rufo-unifasciata; apertura sapius paullo angulata, marginibus undique reflexis, distantibus, columellari brevi, subvertical i, basali strictiusculo.

+ Testr sapissime nitida, glabra (perraro obsoletissime gramulata vel sparsissime pilosa), apertura rotundatorel ovato-lunaris, marginibus breve reflexis.

*) Testa non spiraliter striata, anfractus ultimus antice lente descendens, margines aperturce remoti.

147. H. planospira Lam. (1822): t. depressa, cormea, nitida, in albida vitta rufo-unifasciata, sæpe radiis latis luteis transversis ornata; spira subplana vel convexiuscula; apertura rotundatolunata, basi angulata, peristomate reflexo, margine basali subrecto; diam. maj. 29, min. 24, alt. $13 \mathrm{~mm}$. (Illyria, Tirolia merid., Ital. bor. et Croatia.) Testa late umbilicata, raro lutescente-olivacea, solida, striata, subtus pallida, superius fascia altera rufa, diluta, cito eranescente, anfr. $5 \frac{1}{4}$, planiusculi, ultimus supra depressiusculus, infra convexus, antice breviter deflexus, apertura horizontalis, peristoma albo-labiatum, margine basali vix arcuatulo, columellari subverticali dilatato, partem plus minus majorem aperturæ tegente. Syn. H. umbilicaris Brum. 1838, H. umbiticaris var. Italica Stab. 1864. Fig. Kob.-Rossm. Icon.f. 1057. Variat 1) major: d. 31-33, a. 13-14 mm.; 
2) minor: d. 27 , a. $12 \mathrm{~mm}$; 3) minima: d. 23 , a. $12 \mathrm{~mm}$; 4) depressior: d. 26-27. a. $11 \mathrm{~mm}$.

- var. ctrusca Koв. (1876): t. depressa, sub lente granulata, anfractibus primis interdum pilosis, peristomate latius reflexo, patulo; d. 27, a. $11 \mathrm{~mm}$. (Ital. bor. et media.) Fig. Kiob.-Rossm. Icon. f. 1059. Priccipue umbilico lato ab H. setosula distincta.

- var. ullepitschi mh.: t. depressa, solidior, apertura transversim ovalis, margine columellari in umbilicum immerso; d. 32 , a. $16 \mathrm{~mm}$. (Carinthia.) Fig. Kob.-Rossm. f. 1058.

- var. pactana STג1. (1864); t. plus minusve sulglohosodepressa, spira convexa, anfr. ultimus rotundatus, apertura minus horizontalis, magis rotundata, marg. basali arcuato, columellari late dilatato, umbilicum angustiorem usque ad tertiam partem tegente; d. $25-27$, a. 13-14 mm. (Hatiu bor.) Syn. II. planospira Rossm. Icon. f. 90, 503. (II. hispana Lin. 1758?) Fig. Kob.-Rossm. Icon. f. 1055, 56. Testa persipe pallide cornea, fasciis subevanescentibus, sed variat olivaceo-lutescens, faseiis distinctis.

148. H. argentellei Ков. (1872): t. globuloso-depressa, rufescenti-cornea, fascia peripherica castanea pallide limbata cincta, parum nitidula; spira convexiuscula; apertura rotundato-Iunata, peristomate parum incrassato, margine supero recto, basali arcuato; diam. maj. $18-221 / 2$, min. $15-191 / 2$, alt. $9-11 \mathrm{~mm}$. (Gracia in monte St. Elia Taygeti.) T. mediocriter umbilicata, iepressa, ruguloso-striata, sapius subtilissime granulosa, anfr. 5, convexiusculi, sutura mediocri, subinde albescente, juncti, ultimus rotundatus, apertura valde obliqua, rotundato-lunata, peristoma album, margine supero recto, externo et basali breviter expansis. Fig. Kob.-Rossm. Icon. f. 1053.

149. H. kollari Zer. ap. Pfr. (1856): t. depressa, obscure cornea, fascia rufa utrinque albido-marginata ornata, sparse pilosa; spira vix elevata; apertura late rotundato-lunaris, peristoma album, sublabiatum, marginibus conniventibus, basali subrecto; diam. $17--23$, alt. $7-9 \mathrm{~mm}$. (Serbia.) T. perspective umbilicata, nitidula, striatula, anfr. $5 \frac{1}{2}$, convexiusculi, uitimus depressus, basi pallidus, apert. marg. columellari sat elongato, subverticali, parum dilatato, basali reflexo, externo breviter expanso. líob.-liossm. lcon. f. $1054 .$.

150. H. zonata STUd. (1820): t. globosa, olivacea, fusco unifasciata in vitta allida extus vix conspicua; spira convexa; apertura rotundata, lunaris, marginibus aque curvatis; diam. 22, alt. $12 \mathrm{~mm}$. (Gall. mer.-orient., Helv., Ilatia bor.) 'T. tenuis, me- 
diocriter umbilicata, nitidula, tenuiter striata, anfr. 5--6, convexiusculi, ultimus maximus, inflatus, rotundus. Syn. planospira Mich. 1831. H. foetens M. Tand, 1865. Fig. Rossm. Icon. f. 91. Variat 1) minor: d. 17-20, a. $10 \mathrm{~mm}$. (H. foêtens Stud.); 2) major: d. 25, a. $14 \mathrm{~mm}$.

- var. flavovivescens Dum. \& MorT. (1852): t. solidiuscula, vix pellucida, flavido-viresceus, absque fascia. (H. foet. v. modesta M. T.)

151. H. pyrenaica Drp. (1805): t. depressa, olivaceo-cornea, efisciata; spira planiuscula; apertura horizontalis, subovato-lunaris; diam. maj. 20, min. 17, alt. $9 \mathrm{~mm}$. (Pyrenace) T. tenuis, sat anguste umbilicata, subregulariter dense striatula, supra nune fere planulata, nunc convexiuscula, pellucida, subolivacea-virescens, anfr. $5-51 / 2$, vix convexiusculi, sutura impressa, peristoma acutum, reflexum, vix incrassatum, carneo vel albo labiatum, margine basali arcuato, columellari dilatato. Fig. Dup. Hist. t. 7 f. 2. Rev. Zool. 1S63. t. 13. f. 11 .

- var. complanata Bourg. (1863): to tenuior, magis depressa, sutura profundior, apertura magis elongata et depressa, margine columellari horizontali subrecto. Fig. Rev. Zool. 1863. t. 13 f. 12-14. (Rossm. Icon. f. 218 non bona: $B \mathrm{~g}$.)

152 ? H. subzenata Mouss. (1859): t. orbiculato-convexa, obscure cornea, fascia pallidiori dorsali sub altera obscura ornata, granulis minutissimis obsolete piliferis late inserta, spira convexa; apertura lunato-rotunda; diam. maj. 23. min. 19, alt. $14 \mathrm{~mm}$. (Cephalonie et Epiriss.) T. umbilicata, sutura impressa, anfr. 5, regulares, convexiusculi, ultimus pilis defectis, apertura transverse vix latior, peristoma albidum, breviter reflexum, subincrassatum, marginibus subapproximatis, basali arcuato. Variat diam. 20-26 mın. Cfr. H. setosulam Brig. quacum Kobelt hane formain conjungere velit.

153. H. quimperiana FÉR. (1821): t. planorbica, efasciata, olivaceo-cornea; spira omnino plana vel subconcava; apertura late lunata, marginibus longe remotis; diam. 26, alt. $11 \mathrm{~mm}$. (Gallia prov. Bretagne, Asturia.) T. subaperte umbilicata, haud nitida, tenuis, subtiliter striata, spira angusta (in præcedentibus magna), anfr. 5 convexi, ultimus rotundatus, inflatus, transversim pluries luteostrigatus, apert. peristomate reflexo, albo-labiato, margine basali arcuato. Syn. $H$. corisopitensis Desh. 1831, H. Kermorvani Coll., Mich. et Rossm. Fig. Rossm. Icon. f. 220. Variat 1) major: d. 30, a. 12 min.; 2) minor: d. 20, a. $10 \mathrm{~mm}$.

$$
\text { (II. setosula var.) }
$$

**) Testa sub lente longitudinaliter striata, anfractus ultimus antice forte deflexus, margines apertura approximati. 
154. H. foetens C. PFr. (1828): t. aperte umbilicata, nitida, transversim striatula et sub lente striis tenuissimis et densissimis brevibus, longitudinaliter notata, (corneo-flavescens vel) olivacea; diam. maj. 33, min, 20, alt. $11 \mathrm{~mm}$. (Hetretiu, Austria.) T. convexiuscula, anfr. 5, planiusculi, sat forte acerescentes et latinsculi, ultimus rotundatus, albido-cinctus, superne fascia fusco-brunnea ornatus, apertura rotundato-lunaris, perobliqua, peristomate simplici, acuto, margine basali reflexo, albo. Fig. Rossm. Icon. f. 92.

- var. cisalpina STAB. (1S6t): t. pertenuis, pellucida, virescenti-olivacea, evittata, fusco-unifasciata, anfr. ultimus dilatatus, subtus convexus, apertura horizontalis, ovata, marginibus callo tenuissimo jumctis, exteriore patulo albo, intus rufo-brumneo, basali reflexo et columellari dilatato umbilicum ad $1 / 3$ tegente albo; $d$. maj. 26-27, min. 23, alt. 11-12 mm. (Alpes meivid.) syn. H. vittatr Jan 1832, H. sebinensis Kob. 1873. Variat testa crassiori, badia, depressiuscula, fascia pallida, nulla vel inconspicua (II. rhrtica Mouss. teste Strob.: Stab.) Variat minor: t. anguste umbilicata, d. 18-19, a. $8 \mathrm{~mm}$. (Ital. in prov. Bergamo: Adami ex.) Fig. Kob.-Rossm. Icon. f. 1052.

- var. adamii Ков. (1875): t. latius umbilicata, circa umbilicum depressa, solidula, brunneo-olivacea, fascia angusta, diluta vel obsoleta; d. maj. 32, min. 28, a. $18 \mathrm{~mm}$. (Cirea Lago di Iseo.) Precell, variet. proxima. Fig. Kob-Rossm. Icon. f, 1052.

- var achutes Z. ap. Rossir. (1835): t. minor, nitidissima, brunneo-olivacea, convexiuscula, anfractu ultimo obsolete angulato, zonula lata lactea, superne fascia suturate fusca terminata; d. maj. 18-19, min. 15-16, a. $9 \mathrm{~mm}$. (Tirotia, Carniolia, Anstrin.) Syn. H. cingulina Desh. ap. Fér., H. achatina Pot. \& Mich. 1838. Fig. Rossm. Icon. f. 600 .

- var. ichthyoma HeLd (1837): t. aperte umbilicati, nitidissima, flavescenti-cormen, depressa, spira sæpe subplana, anfractu ultimo obtuse angulato, albo-vittata, superne fascia rufobrumea ornata, apertura perobliqua, depresso-ovata, lunata, albo-labiata; d. ima. 22, min. 18, a. $8 \mathrm{~mm}$. (Bavaria merid., Tirolia, Helv., Austria, Styria.)

If Testa opaca, subtilissime gramulata, apertura subsecurifomis, marginibus late reflexis.

155. H. macrostoma Mühlf. ap. Rossu. (1836): t. aperte mmbilicata, solidula, flavescens, rufo-unizonata; peristoma albolabiatum; diam. maj. 29-33, min. 23-28, alt. $12-14 \mathrm{~mm}$. (Sicilia.) T. orbiculato-depressa, superne subtilissime grauulata, anfr. 
5, vix convexiusculi, ultimus magnus, supra depressus, subtus convexus, rotundatus, apertura magna, lunaris vel securiformis, margine supero sursum porrecto, exteriore forte arcuato, palato subincrassato. Syn. H. planospira Phil., H. siculina Z., pervia et didyma Mühlf. Fig. Rossm. Icon. f. 216. Variat micolor lutescens, solida, absque fascia.

- var. cryptozona Z. ap. Rossm. (1836): t. minor, tenuis, diaphana, obsolete granulata, anguste et pallide unifasciata, palato non incrassato; d. 26 , a. $11 \mathrm{~mm}$.

156. H. confusa BEN. (1857): t. anguste umbilicata, solida, badio-cornea, in vitta lutea badio-unifasciata; peristoma crassum, flexuosum, margine basali calloso; diam. maj. 33, min. 27, alt. $16 \mathrm{~mm}$, (Sicitia.) T. orbiculato-depressa, plus minus granulata, "flavescenti brunnea, fasciis 3 obscuris (lineis flavidis interpositis) ornata, exterioribus latioribus extus dilutis" (Kob.), anfr. 5, convexiusculi, ult. rotundatus, apertura lunari-rotunda, palato subincrassato. Fig. Kob.-Rossm. Icon. f. 106t. Sequenti peraffinis! Variat minor: d. maj. 25-27, min. $21-23$, a. $12-13 \mathrm{~mm}$. (Kob.)

(I. planospira Lam. var.)

10) Hivta: Testa aperic umbiticata, dense hispida, pilis regulariter dispositis, his raro detritis dense granulata vel alutacea, tenera, rufo-unifasciata, in albida vitta fasciis dilutissimis terminata; apertura marginibus anguste reflexis, distantibus vel remotis.

i Anfractus ultimus infra convexus, subtumidus.

157. H. setosula Bric. (1825): t. setosa, setis rigidis, erectis, brevibus, sat caducis; apertura rotundato-lunaris, peristomati reflexo, albo-labiato, margine basali curvato; diam. maj. 25-27, min. 22, alt. $12 \mathrm{~mm}$. (Italia merid.) T. aperte umbilicata, depresso-convexiuscula, alutacea, fusco-cornea, in albida vitta fusco-unifasciata, superius altera fascia pallide fusca, anfr. $5 \frac{1}{2}$, planulati, apert. paullo securiformis. Syn. H. setipila Z. ap. Rossm. 1835. Fig. Rossm. Icon. f. 89. Variat depilata, dense granulata (var. calva Kob. in Rossm. Icon. f. 1060.) Cfr. H. subzonatam Mouss.

158. H. comephora Bourg. (1853): t. dense setosa, setis erectis, rigidis, longis, bene persistentibus; apertura lunato-rotundata, peristomate acuto, superne recto, inferme reflexo, curvato; diam. 27, alt. $13 \mathrm{~mm}$. (Morea.) T. umbilicata, subdiaphnna, fulvo-cornea, convexiuscula, superius fascia pallide fusca ornata, anfr. 5 , planulati, apertura perobliqua. Fig. Rev. Zool. 1857. t. 16. f, 1-4, Kon.-Rossm. Ienn. f. 1063. 
- var. benecticta Ков. (1872): t. spira omnino plana, apertura horizontali dilatata;-d. maj. 29, min. 24, alt. $15 \mathrm{~mm}$. (Sicilia.) Fig. Mal. Bl. XrX. t. 4. f. 4 -6. KKob.-Rossm. Icon. f. 1062.

159. H. hirta MeNke (1828): t. hirta, pilis brevibus, densissimis, rigidis; apertura late lunata, margine basali recto cum columellari brevi angulum rectum formante; diam. maj. 20-21, min. 17, alt. 8-9 mm. (Illyria, Croatia.) T. aperte umbilicata, depressa, alutacea, pallide lutescenti-cornea, rufo-unifasciata, anfr. $5 \frac{1}{2}$, planulati, peristoma reflexiusculum, subincrassatum, candido-labiatum. Fig. Rossm. Icon. f. 599.

ti Anfractus ultimus subtus pone aperturam et in eadcm peripheriam versus compressus.

160. H. lefeburiana FÉr. (1819): t. pubescens; peristoma albolabiatum, reflexum, margine basali curvato, columellari dilatato; diam. maj. 29, min. 24, alt. $13 \mathrm{~mm}$. (Illyria.) T. umbilicata, planospira, cinereo-cornea, distincte unifasciata, tenuis, pilis brevibus, sat erectis, mollibus olssita, anfr. 5, planulati, sutura sat profunda, apertura perobliqua, rotundato-vel late lunaris. Fig. Rossm. Icon. f. 94, 501,

161. H. sadleriana ZIEGL. ap. Rossm. (1838): t. pilis brevissimis rigidis dense obsita; peristoma subincrassatum, albidum, reflexiusculum, margine basali strictiusculo, columellari latissime dilatato, reflexo; diam. 28-30, alt. 12-14 mm. (Croatia, Carniolia.) T. late umbilicata, tenera, nitida, pallide lutescente-cornea, distincte rufo-unifasciata, utrinque fasciis dilutissimis, anfr. $5^{1} / 2$. planuIati, apert. rotundato-lunaris. Fig. Rossm. Icon. f. 502.

11) Cingulatc: I'esta umbilicata, albida, temus at firma, niticla, lavigata rel striatula, fusco-unifasciata; anfr. ult. antice deflexus; apertura ovata, marginibus coniergentibus, columellari elongato, basali arcuato, supra umbilicum affixo, reflexis, externo subrecto.

+ Testa major, peristomate cxpunso, reflexo.

162. H. preslii Schm. ap. Rossu. (1836): t. depressa, spira sæpius subplana, striata, dilute subtrifasciata; anfr. ultimus supra depressus, subtus convexus; apertura ovato-rotundata; diam. 25-27, alt. $10 \mathrm{~mm}$. (Illyria, Tiroliu, Bararia merid., Italia, Turcia.) 'T. late umbilicata, cinereo-carnea, subtus albida, anfractus 5, planulati, ultimus sicut obtuse angulatus, fascia angusta castanea supra et altera maxime diluta paralleli infra medium, rarius superne tertia ornata, apert. perobliqua, swepius intus brunnea, peristoma di- 
latatum, reflexum, sublabiatum. Syn. H. cingulata Held. 1847. Fig. Rossm. Icon. f. 225 .

- var. nisoric Rossu. (1838): t. minor, fascia media distincta, utrinque late albo-marginata, lateralibus variegationem latam , maculosam dissolutis; d. 20-22, a. $9 \mathrm{~mm}$. -- Fig. Rossm. Icon. f. 509. - Obs. Cel. Gentiluomo in Bull. mal. ital. 1868 "H. cingulatan mut. anconse" descripsit ("t. minor, fusco-trifasciata, supra planulata, inferne valde convexa, umbilico expanso, apert. subrotunda, anfr. 5 planulati") et t. 3. f. 9-11 depinxit, quæ a clar. E. v. Martens ad H. preslii, a clar. Pfeiffer ad H. chamæleon ducitur.

163. H. cingulata Stud. (1820): t. convexa, striatula, fuscounifasciata; anfractus ultimus rotundatus, convexus; apertura subrotunda; diam. 26 , alt. $13 \mathrm{~mm}$. (Tirotia, Illyria, Italia superior.) T. mediocriter umbilicata, griseo-cornea, circa umbilicum albida, anfr. $51 / 2$, convexiusculi, apertura intus concolor, peristoma sublabiatum, acutum, marginibus approximatis, supero expanso, basali reflexo, columellari dilatato. Fig. Rossm. Icon. f. 87. Variat 1) unicolor, albida, absque fascia, apertura ampliore, perist. minus reflexo (Rossm. Icon. f. 371); 2) minor: d. 21, a. 10-11 mm. (Rossm. f. 372).

- var. anauniensis BETTA (1852): t. sæpius multo minor, subpellucida, brunnescens, fascia lata, castanea.

- var. Inganensis Scmistz: t. depressior, pallide straminea (supra fuscula), zona obscure brunnea. Mutationem, philippimaria nominatam (fascia angusta pallida, longitudinaliter reque bipartita, d. 23 , a. $10 \mathrm{~mm}$.), ad Lugano invenit clar. Stabile (Bull. mal. ital. 1868. t. 2. f. 7.)

- var. appelii Ков. (1876): t. suborbicularis, depressa, umbilico latissimo, perspectivo, fascia lata, rufo-brunnea, apertura rotundata; d. maj. 30, min. 25 , a. $13 \mathrm{~mm} ., \mathrm{umb} .6^{1 / 2}-7 \mathrm{~mm}$. lat. (Laicca.) Fig. Kob.-Rossm. Icon. f. 1070.

- var. carrarensis Ponno (1869): t. depresse subglobosa, anfractibus inflatis, umbilico angusto subcylindrico, pallida, fascia obscure brunnea submediana, utrinque fascia multo pallidiore et angustiore, apertura subcircularis; d. 25, a. $13 \mathrm{~mm}$. (Ital., Carrara.) Fig. Kob.-Rossm. Icon. f. 1071. Var. apuana Iss. (in Alp. apuan.): t. magis convexa, fortius striata.

- var. colubrina $J_{\mathrm{AN}}(1830)$ : t. albida, maculis corneis (vel cornea, maculis albis) marmorata, pertenuis, hyalino-pellucida, fascia angusta pallide brunnea, marginibus aperturæ disjunctis; $d$. 23 , a. $10 \mathrm{~mm}$. Fig. Rossm. f. 370, 1074. Variat 1) minor, spira magis elevata, umbilico angustinre; ๙. 24 , a. $15 \mathrm{~mm}$. (Tirolia ad Riva.) 
Fig. Kob.-Rossm. f. 1077; 2) fascelinci Z.: t. solida, fasciis lateralibus dilutis sed distinctis, media obsoleta vel nulla, anfr. ult. tumidus, apertura magna, margine inferiore arcuato; d. maj. 30 , min. 25 , a. 15 mm. (Tirolia ad Riva, Italia ad Mantua.) Fig. Kob.-Rossm. Icon. f. 1075; 3) mubila Z.: t. colore colubrinæ typicæ, apertura dilatata, perobliqua, infra subplanata, margine basali substricto; d. maj. 27, min. 23, a. 12-14 mm. (Ital. super.) Fig. Kob.-Rossm. Icon. f. 1073.

- var. bizona Rossu. (1842): t. lactea, fascia altera inferior diluta, apertura rotundior, minus obliqua, marginibus remotioribus; d. 24, a. $12 \mathrm{~mm}$. (Nizza.) Fig. Rossm. f. 683.

- var. baldensis ViltaA (1841): testa major, umbilico latiore, marginibus apertura subconnexis; d. 29, a. $13 \mathrm{~mm}$. (ATonte Baldo Tivolice.) Fig. Rossm. f. 603, 604.

\section{ti) Testa minor, peristoma margine supero recto.}

164. $H$. cingulella Z. ap. Rossm. (1837): t. depressa, subplana, striatula, transverse livido-radiata, fusco-unifasciatn; anfr. ult. superne obsolete angulatus, infra convexus; apertura ovata; diam. 16-18. alt. 6-7 mm. (Vollynia.) T. late umbilicata, nitidula, sordide alba, fascia perangusta, anfr. 5, depressi, ultimus circa umbilicum altior, extus compressus, apert. margine dextro subrecto, simplici, basali reflexiusculo, sublabiato, columellari subuliatato-patente. Fig. Rossm. Icon. f. 373. Variat (teste Rossm.) 1) unicolor, absque fascia; 2) t. fascia pallida; 3) gyrata: t. fasciis 2 superis et $3-4$ inferis, obsoletissimis.

165. H. nicatis Costa ap. Prr. (1853): t. depressa, spira subplana, subarcuato-striata, albida, maculis corneis obsolete adspersa, pallide rufo-unifasciata; anfr. ult. infra convexior; apert. lumato-potundata, intus fusco-cornea; diam. 20; alt. $8 \mathrm{~mm}$. (Ital. mov. Neapolitana.) T. latius umbilicata, anfr. 5, planiusculi, ult. antice vix descendens, peristoma simplex.

166. H. zonella PFr. (1864): t. convexa, conferte rugulosostriata, pallide fuscula, zona mediana albida (sæepe rufo-marginata); anfr. ultimus rotundatus; apertura lunato-rotundati, intus margaritacen; diam. 15, alt. $8 \mathrm{~mm}$. (Crela.) T. subnouste umbilicata, soliclula, anfr. 51/2, convexiusculi, ult. antice vix descendens, peristoma simplex

12) Crinita: Testa albida, scpius bifasciata, globoso-demessa, striata; anfractus ultimus antice valde deflexus; aperture perobliqua, transverse malis rel subcirculuris, marginibus subconnexis, vel contimis solutis, peri- 
stomate reflexo, columellavi subdentato rel calloso.

$\dagger$ Testa villosa vel hispida.

*) Testa angustius ct semioblecte umbilicuta.

167. H. hoffmanni Partsch ap. Rossm. (1856): t. depressoglobosa, albido-lutescens, rufo-unifasciata; apertura rotundato-ovalis; diam. 28-31, alt. 15-18 mm. (Dalmatia, Montenegro.) T. sub lente punctato-granulata, tenera, subpellucida, hispida, pilis densissimis et brevissimis, raro obsolete trifasciata, anfr. $51 / 2$, convexiusculi, ult. magnus, rotundatus, apert. perobliqua, peristoma latum, reflexum, sublabiatun, marginibus subconniventibus, non continuis, columellari dilatato, umbilicum semitegente. Syn. H. monozona Z. Fig. Lessm. Icon. f. 224. Variat major: d. 35, a. $22 \mathrm{~mm}$. (Brus.)

168. H. kleciachi Parr. ap. Pfr. (1870): t. globoso-clepressa, lutescenti-cornea, fasciis tribus subrqualibus fuscis ornata; apertura transver'se ovalis; diam. 30, alt. $15 \mathrm{~mm}$. (Dalmatia, Bosnc.) T. minute et confertissime granulata, pilis brevibus, erectis obsita, anfr. 5, convexi, ultim. depresso-rotundatus, apert. perobliqua, peristoma albo-labiatum, marginibus callo sinuoso junctis, basali supra umbilicum mediocrem dilatato, patente. Testa bosnensis est (teste clar. Möllend.) globosior, dente aperturæ obsoleto. Syn. H. luccscens Kutsch. ap. Brusina 1866 (nomen.) Fig. Kob.-Rossm. Icon. f. 1065.

**) Testa aperte vel late umbilicata.

169. H. crinita SANdri ap. Strob..(1858): t. depressa, irregulariter rugosa, villis longis, raris, regulariter dispositis obsita; apertura rotuniata, peristomate continuo, soluto; diam. 28, alt. 12-13 mm. (Dalm., Bosna.) T. aperte umbilicata, sub lente valide granulata, solidula, subpellucida, nitida, fusco-cornea, pallide rufotrifasciata, anfr. $5 \frac{1}{2}$, planulati, apert. lata, peristomate lato, reflexo, labiato. Fig. Kob.-Rossm. Icon. f. 1068.

170. H. sotosa Z. ap. Rossu. (1836): t. depressa, subtiliter striato, villis densis longiusculis irregulariter dispositis hirsuta; apertura rotundata, peristomate continuo, soluto; diam. 30, alt. 15 mm. (Dalm., Istria, Croatia, Albania, Bosna.) T. late umbilicata, sub lente subgranulata, tenera, pellucida, lutescenti-cornea, rufo trifasciata, anfr. $5 \frac{1}{2}$, convexiusculi, peristoma latum, reflexum, sublabiatum. Fig. Rossm. Icon. f. 221. - Variat 1) major: d. 35, a. 16 mm.; 2) minor: d. 23, a. $11 \mathrm{~mm}$. (Fig. Rossm. Icon. f. 605--606); 3) convexior: d. 28, a. 17 mm.: 4) albina, efasciata; 5) litoralis Bruls. (1869): magna, fasciata, brevissime villosa, magis rugosa et opaca (Dalm.)

171. H. setigera Z. ap. Rosssr. (1836): t. depresso-globosa, subtilissime striata, villis densis longiusculis, subregulariter dispo- 
sitis obsita; apert. rotundato-ovata, peristomate continuo; non soluto; diam. 33-26, alt. $11-13 \mathrm{~mm}$. (Dalm.) T. sat anguste umbilicata, sub lente granulata, tenera, pellucida, lutescens, pallide trifasciata, fasciis lateralibus plerumque evanescentibus, anfr. 5, convexi, peristoma reflexiusculum. Fig. Kob.-Rossm. Icon. f. 1069. Variat 1) albina, unicolor; 2) minor: d. 21, a. $13 \mathrm{~mm}$; 3) globulosa Kac., minor, globulosa, d. 20, a. $11 \mathrm{~mm}$. (Ins. Meleda.)

172. H. brusinæ Stoss. (1876): t. planulata, subtiliter striata, villis brevibus densissimis subregulariter dispositis obsita; apert. rotundata, peristomate continuo soluto; diam. 32 , alt. 15 $\mathrm{mm}$. (Monte Biokovo.) T. late umbilicata, sub lente granulata, tenera, subpellucida, lutescente-albida, fusco-rufo trifasciata, apert. ampla. peristomate lato, reflexo. Fig. Bull. Soc. Adriat. I. p. 293.

\section{t广 Testa glabra.}

173. H. insolita Z. ap. Rossy. (1838): t. aperte umbilicata, globoso-depressa, striata, sub lente conferte granulato-lineata; anfr. $51 / 2$, convexi; apertura ovata, marginibus subconnexis: diam. $281 / 2$, alt. 15-16 mm. (Dulm.,Bosna.) T. tenera, nitida, pallide lutescens, fusco-trifasciata, fasciis lateralibus sape evanescentibus, interruptis, peristoma reflexum, subincrassatum. Syn. $H$. insolida Pfr. \& alior. Fig. Rossm. Icon. f. 506 . Variat: d. 32 , a. $17 \mathrm{~mm}$.

174. H. denudata Rossm. (1836): t. late umbilicata, convexiuscula, subtiliter striata, sub lente"lineolis confertis, flexuosis ornata; anfr. $5 \frac{1}{2}$, convexiusculi; apertura rotundata marginibus subconnexis; diam. 35, alt. $19 \mathrm{~mm}$. (Dalm.) T. solidula, nitida, ntescens, fusco-trifasciata, fasciis lateral. sapius evanescentibus, interruptis, peristoma reflexum, incrassatum, albidum. Fig. Rossm. Icon. f. 223 .

175. H. prætexta Park. ap. Pkr. (1870): t. mediocriter umbilicata, subconoideo-depressa, confertim subrugoso-striata; anfr. 5, convexiusculi; apertura, ovali-rotundata, marginibus approximatis; diam. $27-30$, alt. $14-16 \mathrm{~mm}$. (Dalm.) T. solidula fulvescenti-albida, depresso turbinata, castanco-trifasciata, fasciis 2 superis angustis, sepe evanescentibus, subtilissime et densissime longitudimaliter striata, anfr. ult. depresso rotundatus, antice sensim descendens, basi pone peristoma subconstrictus, peristoma sublabiatum, marginibus callo temuissimo vix junctis, basali obsolete tuberculifero. Syn. H. insolida Wald., H. insol. var. vugute Kutsch. ap. Brus. 1866, Cump). nsol. v. costulcta B\%. ap. Bruz. 1869, 11. pretextale Kiob., Mart. Fig. Kol.-Rossm. lcon. f. (1066?) 1067.

176. H. imberbis Brus. (1876): t. late umbilicatil, depressiuscula, sub lente valide granulata; anfr. $5^{1 / 2}$, subplanati; aper- 
tura rotundata, peristomate continuo, soluto; diam. 28, alt. 13 mm. (Croatia.) T. subpellucida, nitida, rufo-trifasciata, peristoma latum, reflexum, sublabiatum. Syn. $H$. denudata $\mathrm{Z}$. et Camp. setosa $v$. denud. ap. Brus. 1867, 69, 70. (Br.)

13). Stenomphala: Testa luiescens, obscure trifasciata, globuloso-depressa, striata, demum

- lamina lata marginis columella obtecte perforata; apertura marginibus distantibus.

177. H. stenomphala Menke (1830): t. anguste umbilicata, globoso-depressa; anfr. ult. antice vix descendens; apertura late lunaris, albo-labiata, margine basali rectiusculo; diam. 31, alt. 19-2.3 mm. (Croatia.) T. subtiliter striata, olivaceo-lutescens, castaneo-trifasciata, fasciis lateral. dilatatis et dilutis, anfr. $5 \frac{1}{2}-6 \frac{1}{2}$, convexiusculi, ultimus inflatus, peristoma reflexiusculum, margine columellari dilatato, reflexo, umbilicum semioccultante. Fig. Rossm. Icon. f. 458.

178. H. raspailli PAYR. (1826): t. obtecte perforata vel imperforata, depressa; apertura lunato-ovalis, peristomate sordide labiato, margine basali elongato, calloso; diam. 34. alt. $17 \mathrm{~mm}$. (Corsica.) T. fusco-lutea, rufo-trizona, striata, solidiuscula, anfr. $4^{1} / 2$, planulati, ultinus antice subdeflexus, sutura brunnco-marginata, apertura margine columellari valde dilatato, reflexo, perforationem demum claudente, sæpius maculo hepatico. Fig. Rossm. Icon. f., 505, Bourg. Moll. nouv, X. 1868, t. 44. f. 1-3.

- brocartina Dur. (1867): t. magis inflata, minus depressa, fortius striata, major, solidior, obseurior, anfr. ult. subtus inflatior, apertura magis rotundata; d. 36, a. $18 \mathrm{~mm}$. - Fig. Bourg. 1. c. t. 44. f. 4, 6, Kob.-Rossm. Icon. f. 986 .

179. H. romagnoli DUT. (1877): t. imperforata, convexiuscula, aspera et eleganter hispidula; apertura vix lunata, transverse ovato-ratundata, peristomate carneo-luteolo, leviter incrassato, margine basali rotundato; diam. 30-33, alt. $14-15 \mathrm{~mm}$. (Corsica circa Corte.) T. uniformiter rufo-olivacea, tribus zonulis castaneis ornata, tenerrima, infra convexa, non nitida, pilis exiguis, subulatis, curvatis aspera, anfr. 5, fere planulati, ultimus maximus, compresso-rotundatus, antice deinde velociter descendens, peristoma undique expansum, marginibus approximatis.

? 180. H. insularis CR. \& DEB. (1869): to imperforata, depressa, viridulo-albida, lineis longitudinalibus raris, pallide olivaceis, parum conspicuis ornata; apertura lunato-ovalis, "peristomate albo; diam. 27, alt. $12 \mathrm{~mm}$. (Corsica supra Cenestinum.) T. te- 
nuissime striatula, solidula, nitidula, anfr. $4-4^{1 / 2}$, ult. antice subito descendens, rotundatus, peristoma breviter reflexum, marginibus subconvergentibus. Fig. Journ. Conch. 1869. t. 2. f. 3. Celeb. Kobelt vult in hac tantum mut. albinam H. rasp. videre.

181. H. revelieri DÉB. (1867): t. semiobtecte umbilicata, depressa, striata, striis-spiralibus subtilissimis, apertura late rotundato-ovata, peristomate simplici, margine basali arcuato; diam. 27, alt. 12 nm. (Corsica in Monte Renoso.) T. tenuis, nitida, fusco-virescens, fasciis rufo-brunneis ornata, anfr. $4^{1} / 2$, ultimus dilatatus, supra planatus, peristoma marginibus approximatis, callo tenui junctis, supero ad insertionem tuberculato. Syn. H. cymiace Dut. 1867, H. plunospira Payrod. Fig. Kob.-Rossm. Icon. f. 987.

B) Xerocampylæa Ков. Testa Aepressa, angustissime umbilicata, marginibus aperture connicentibus, peristomate undique recto, tenuissime labiato. (Animal sagittis umatoriis 2 paullulum curvatis, naxilla tenue undulato-plicatula: Mröllencl.)

182. H. zelebori PrR. (1853): t. conoideo-depressa; apertura ampla, lunato-ovalis; diam. 14, alt. $7-8 \mathrm{~mm}$. (Sevbia.) T. striata, nitidula, albida unicolor vel lineis 2 rufis circumdata, anfr. $5 \frac{1}{2}$, modice convexi, ult. antice vix descendens, apert. intus pallide carnca.

12. Chilotrema LEACrr. Testa aperte umbilicuta, lenticulari depressu, medio ocute carinata, fusco-maculata vel strigata (interdum et typice ad 4 fascinta); anfr. 5; apertura hovizontalis, transverse ovalis; peristoma continurum, solutum.

Ilabitat præcipue per regiones montanas, nunc presertim abundanter in rimis petrarum calcarearum, nunc in saxis lapidusque granitoideis, locis rupestribus silvarum, imo interdum fere semper in trumcis arborum frondosarum, præsertim fagorum. Fossilis in strato pleistocenico supero.

183. H. lapicida Liv. (1745): t. subtilissime granulata, lutescenti-cornea; anfr. ult. antice subito deflexus; diam. 16-18, alt. $6-7^{1} / 2 \mathrm{~mm}$. (Europa.) Variat 1) albine, unicolor, pellucida; 2) major: d. 20 , a. $9 \mathrm{~mm}$; 3) minor: d. 12, a. $5-6 \mathrm{~mm}$. H. Iap. $v$. grossularia Voith, anfractu ultimo rotundato, subecarinato, est monstrositas, et transitus $H$. lecoquii Put. ap. M. T., t. minor, depressa, carina obsoletiorc. Syn. H. affinis Gmel., II. acuta Da C.; Junior, allina $=I I$. albella Lin.! Juvonis $=I I$. explanate Schr. Fig. Rossm. Icon. f. 11. (Foss.) 
13. Arionta Leach. Testa obtecte perforata vel anguste umbilicata, depresso-globosa, unifasciata vel concolor, strigata et spiraliter lineata; anfr. ult. convexus, tumidus; apertura lunato-rotundata, peristomate late labiato.

Ilabitat in hortis, fruticetis et silvis, pracipue in regionibus montosis et elatis. Fossilis in strato pliocenico medio et supero et pleistocenico toto.

184. H. arbustorum LiN. (1758): t. obtecte perforata, fusca, unifusciata et stramineo-aspersa; diam. 21, alt. $18 \mathrm{~mm}$. (Europa.) T. nitida, globosa, dilute strigosa et decussata, anfr. 5-6, convexi, ult. antice vix descendens peristoma reflexum, albo-labiatum. Fig. Rossm. Icon. f. 1 \& 297 a-s. Testa magnitudine, forma et colore maxime variabilis. Variat 1) efasciata; 2) flavescens vel lutescens, subunicolor; 3) pallida, lineis candidis; 4) morbosa-albina Rossm., t. pallide Intea vel flavo-albida, fascia obsoleta v. nulla, colore griseo-flavescente sub epidermide tincta; 5) rufescens, albo maculata; 6) thomasia M. T., grisea, albo-maculata.

- var. picea Z. ap. Rossm. (1837): t. tenuissima, transparens, castanea, unicolor, unifasciata: dian. 20, a. $13 \mathrm{~mm}$. (Norv., Siles., Bohcm., Gall. oricnt., Alpes, Tronssilv., Volhynia, Ital. bor.) Syn. H. vittmanni Zaw. teste Rossm., H. arb. v. fusca M. T., H. arb. v. luctuosa Slav. Fig. Rossm. Icon. f. $297 \mathrm{~d}$.

- var. ulpestris Z. ap. Rossur. (1837): t. minor, solida, fere omnino lutea, unifasciata; d. $18-19$, a. $12-15 \mathrm{~mm}$. (Alpes, Siles., Scandin. bor.) Syn. H. alpicola Fér. Fig. Rossm. Icon, f. 297. b. Variat minor: d. 16, a. $11^{1 / 2}-13 \mathrm{~mm}$.

- var. repcllini Cr.: t. magis depressa, tenuis, pellucescens, pallida; d. 23, a. $14 \mathrm{~mm}$. (Gall., Carinth., Ital. sup.) syn. H. planospira Gras 1810. Moll. Isère, t. 3. f. 11.

- var. conigonensis -Boub. (1833): t. magis depressa, tenuissima, pellucida, brunneo-virescens, obsolete fasciata v. unicolo1. (Pyren. oricnt.; Carinth.) syn. H. xatartii Far. 183t, teste cel. M. Tand. Cl. Pfeiffer hane tormam aliis verbis descripsit: solida, virescenti-flavida, subtus brumnescens, umbilico mediocri subobsoleto: diam. 18, alt. $11 \mathrm{~mm} . "$

- var. mudis MühLF. ap. Rossu. (1837): t. minor, sat depressa, spira planiuscula, umbilicata, rugoso-subcostata, maculis flavis in lineis transversis subconfluentibus; d. 21-23, a. 13-14 mm. Alpes super. ("non infra 1000' s, m."; Dania teste Mörch, 
Norv. ad Frederiksværn.) 'syn, $H$. arb. v. stentzi Rossm. 1835. Fig. Rossm. Icon. f. 297 e. \& f. 78.

- var. calcarea Högr. (1841): t. calcarea, crassa, solida, obscure castanea, luteo-maculata, intus obscure purpurea; diam. 22-24-25, a. 16-18-21 mm. (Suce. ins. Gotland, Dan. ins. Möen.) Ar. arb. var. creticola Mörch 1864: ponderosa, gigantea, diam. $27 \mathrm{~mm}$.

- var. depressa HesD: t. magna, depressa, tenuis, sat perforata, colore formoe typicx; anfr. ult. dilatatus; d. 23-25, a. 15-18 mm. (Silesia, Salzburg.) Variat major: d. 30, a. $20 \mathrm{imm}$.

- var. Hrochoidalis Rofr. (1868): t. trochoidea, spira alta, sxpe subscalaridea; d. 22, a. $21 \mathrm{~mm}$. (Germ. merid., Holv.)

- var. conoidea WesterL. (1871): t. maxima, conica, subperforata, irregulariter subcostulata, unifasciata, maculis pallide luteis ad lineas transversales densas confluentibus, anfr. $61 / 2$, ult. pone aperturam extus fascia lata, pallide lutea; diam. 23-24, a. 25-26 mm. (Suecia ad Lund.)

- var. styriaca Frauenf. (1868): t. depressa, superne levissime convexa, subtus perventrosa, mediocriter umbilicata, corneofusca, fisciata et lutescenti-maculata; d. 25, a. $12^{1} / 2-14 \mathrm{~mm}$. (Styri(t.) Fig. Kol.-Rossm. Icon. f. 988.

185. H. æthiops Bielz ap. PFr. (1859): t. anguste umbilicata, olivaceo-nigra, unicolor; diam. 18, alt. 9 mm.. (Transsilv.) T. nitida, globoso-depressa, tenuissima, irregulariter plicato-striata, anfi. $4 \%$, convexiusculi, ultim. subdepresso-rotundatus, antice non descendens, luteo-limbatus, apert. late lunaris, leviter albo-labiata, marg. colum. supra perforationem dilatato.

14. Xerophila IIELd. Testa umbiticata ( vimato-perforata), turbinato- val globoso-clepressa, culcureu, srepins fusciatu, fasciis 4 (2 superis et 2 inferis), sed persrepe in maculis ed lineis solutis; aperture lumatorotundata vel subcircularis, peristomate acuto, intus labiato.

Habitat locis soli maxime expositis, in campis apricis, in graminosis pratis, pascuis, inter gramina brevissima in locis glareosis, aridis, semper fere in regionibus calcareis, srepe ad litora marium, majores species interdum in herbis, minores in muris, lapidum acervis etc. Fossilis a strato miocenico infero. 
1) Eupurypha Hartu.: testu dense spiraliter lineata (quod numquam in sectionibus scquentibus.)

† Testa depressa, subplana, anfr. ult. acute carinatus, apertura angulata.

186. H. gualtieriana Lin. (1767): t. superne plana, decussatim plicata, lineis spiralibus præsertim elevatis; anfr. $41 / 2$, ult. antice valde deflexus; apert. lunato-securiformis, latitudine paullo major, intus lutescens, peristomate albo-incrassato; diam. 43, alt. $18 \mathrm{~mm}$. (Hispania.) T. impérforata, cinereo-lutescens, inoniliformiscabra, subtus turgida, compresso-carinata, peristoma simplex, margine superiore recto, acuto, basali forte arcuato, reflexo, columellari apresso. Fig. Rossm. Icon, f. 237, 809-811.

- var. laurenti Boung. (1870): t. supra convexi-tectiformis, valide crispato-sulcata, striis angustis et spiralibus decussata; apert, leviter lunata, fere xque alta ac lata, marginibus callo junctis; diam. 34, alt. $19 \mathrm{~mm}$.

187. H. planata Chems. (1795): t. supra plano-convexiuscula, sub lente dense undulato-striata et decussata; anfr. ult. antice non descendens; apertura angulato-lunaris, latitudine multo miijor, intus rosea, rubro-labiata; diam. 20, alt. 9-10 mm. (Tauria: Kryn.; Afr. bol.). T. obtecte perforata, alba, unicolor vel lineis fuscis et rubellis varie cincta, vel supra alba, infra lutea, spira parum elevata, anfr. 5, plani, ultimus acute carinatus, basi turgidus, apert. marginibus callo introrsum diffuso vinoso junctis, basali levissime arcuato, incrassato. Syn. $H$. helicella Wood, $H$. calliostoma Ad. \& Rv. Fig. Chemn. Cab., Hel. t. 21, f. 10-12.

- var. arietina Rossic. (1846): t. perforata, albida, fasciis luteis latis et lineis fuscis ornata, anfr. ult. antice descendens, carina compressa, apert. Iunato-securiformis, valide labiata, minus transversalis; d. 21, a. $12 \mathrm{~mm}$. (Hispan. merid.) Fig. Rossm. Icon. f. 825 .

if Testa subglobosa, anfr. ult. convexus, rotundatus, apertura rolundato-lunata.

188. H. pisana MüLL. (1774): t. anguste perforata, vix irregulariter striata, subtilissime spiraliter lineata, diam. 20, alt. $14 \mathrm{~mm}$. (Regio mediterranea, Anglia.) 'T. sat tenuis, sed solidula, candida, lutescens vel grisea, diversissime castaneo-fasciata, tæniata, maculata vel punctata, anfr. 5, convexi, peristoma lectum, roseo-labiatum, margine columellari reflexo. Syn. H. zonaria Penn. 1777, Coch. 
virgata Da C. 1778, H. petholate Oliv. 1792, II. rhodostoma Drp. 1801, H. cingenda Mont. 1803, H. strigata Dillev. 1817. Fig. Rossm. Icon. f. 359. - Variat 1) globosior Sh. 1843: t. minor, globosa; 2) delvessa Req. 1818: t. depressa; 3) maculata Mke 1830: t. depressa, plus minus carinata; 4) sardoc Z. ap. Nke 1830: t. minor, umbilico subclauso, fasciis continuis et interruptis, d. 12-13, a. $9 \mathrm{~mm}$. (Sardoa, Gall. austr.); 5) spheroidea: t. major, sphæroidea (Rossm. f. 614); 6) alboranensis IV. \& B.: t. minor, subdepressa, umbilico subclauso (Cadix, Lissabon.)

2) Helicella M. T.: testa aperte vel, late umbilicata, depressa, non carinata; anfr. ult. tereti-convexus; apertura lunari-rotundata.

\section{+ Testa lavigata vel irregulariter striatula.}

189. H. cespitum Drp. (1801): t. aperte et parum umbilicáta, globoso-depressa, irregulariter striata, albida, plerumque fusco-multifasciata; anfi. ult. magnus, teres, vix descendens; apertura albo-labiata; diam. 20, alt. $10-11 \mathrm{~mm}$. (Gall., Ital., Sicil., Lusit., Hisp. \&c.) T. opaca, a lactco ad purpureum colorem transiens, unicolor, fasciata vel tæniata, spira parum elevata, obtusa, anfi. 6, convexinsculi, apert. lunato-rotundata, marginibus remotis, conniventibus. Syn. H. itala Lin.; H. fasciolata Poir.? Fig. Rossm. Icon. f. 513 (typ.), $514-516$ (varr.) - Variat 1) major: d. 25-28, a. 14-18 min.; 2) minor: (l. 15, a. $8 \mathrm{~mm}$. ; 3) albo-fasciata, testa fusco-cornea, minor (Sicil.)

- var. introducta Z.: t. magis depressa, sæpissime unicolor, lutescens, vel fascia una supra mediana, apcitura peristomate expansiusculo, umbilicus latior. (Ital. bor., Gall. ad Grasse.)

190. H. arigonis Rossu. (1854): Species characteribus anatomicis prxcipue a præcedente diversa; testa differt anfractu ultimo ad suturam semper depresso (non convexo), umbilico angustiore (non $1 / 4$ diam.), apertura latiore quam alta (non vice versia), labio unico (non labiis $1-3$ ), colore multo obscuriore. (Hisprenia.) Fig. Rossm. Icon. f. 823, 824.

191. H. neglecta Dre. (1805): t. aperte umbilicata, glubosodepressi v. depressa, supra albida; anfr. ult. rotundatus, non descendens; apert. sub-rufo labiata; diam. 14-18, alt. $7-9 \mathrm{~mm}$. (Gull., Ital. bor., Hisp., Gicecia.) 'T. solida, tenuissimo irregulariter et remote striatula, infra srepe fulvo-rubescens, fasciis 1-6 continuis vel interruptis ornata, anfr. 5-6, convexiusculi, apertura subcircularis, parum lunata, peristoma rectum, acutum, narginibus conniventibns. Syn. II. clivorum IIartm., H. varians Risso ex. Pfr. Fig. Rossm. Icon. f. 355. Vuriat 1) colore: alba unicolor, fusca, luteseens rel ochrolenca, vario modo fasciata; 2) magnitudine: major; d. 20, a. 
$20 \mathrm{~mm}$. \& minor, d. 8-9, a. 5-51/2 mm., 3) forma: spira depressa \& spira suliconica.

- var. submeglecta Boung. (1860): t. magis cretacea, solidior, spira elatiore et umbilico paullo minus aperto. (Ins. Charteau d'If.)

- var. fagoti mh.: t. aperte umbilicata, spira conica, anfr. ult. medio obsolete angulatus, apextura ovalis, marginibus distantibus. (Gall., Villefranche; spec. cl. Fagot communicavit.) Variat colore et magnitudine.

192. H. nubigena Cri. ap. SAulcr (1852): t. aperte umbilicata, globoso-depressa, albidula, efasciata, ad aperturam luteola seu rosea; anfr. ult. rotundatus, vix descendens; apertura intus albido-incrassata; diam. 11, alt. $6 \mathrm{~mm}$. (Pyrencere prope Baréges etc., Italia in Aprutiis.) T. striatula, anfr. 5, cenvexiusculi, altimus rotundatns, apertura rotundata, peristomate acnto, marginibus approximatis. Fig. Journ. Conch. 1853. t. 3. f. 7.

193. H. enhalia BouRG. (1865): t. aperte umbilicata, globososubdepressa, alba, sæpius fusco-multifasciata; anfr. ult. inflatus et rotundatus; apertura intus minime marginata; diam. 7-9, alt. 5-7 mm. (Gall. ad Biarritz.) T. tenuis, subopaca, tenuissime striata, anfi. 5-6, convexi, apert. perfecte rotundata, peristoma simplex, ncutum. Syn. H. arenosa Dup: 1819, non Z. Fig. Hist. t. 13. f. 9.

194. H. ericetorum MüLl. (1774): t. late v. latissime umbilicata, depressa, lutescenti-alba v. cinerea, fulvo-fasciata; aufr. ult. cylindricus, antice deflexus; apertura intus leviter labiata; diam. 17 ; alt. $8 \mathrm{~mm}$. (Europa occidentalis; orient. versus in Germ. mer. ad $28^{\circ}$ et Germ. bor. ad $34^{\circ}$.) T. tenuis, striata, cornea, fasciis raro filiformibus, rarissime interruptis, parum nitida, anfr. 6, convexiusculi, sutura profunda, apertura rotundata, marginibus subapproximatis. Syn. H. itala Lin.?, H. media Gmel.?, H. dubia Hartm., H. zonaria Schr., H. albella Penn., H. erica Da C., H. umbiticaris Oliv., H. küsteri Held. Fig. Rossm. Icon. f. 17, 517. - Variat 1) major: d. 20, a. $10 \mathrm{~mm}$. (in Gall. d. 25, a. $12 \mathrm{~mm} .:$ Dup.); 2) minor: d. 11, a. $5^{1 / 2} \mathrm{~mm}$.

- var. devians WesterL. (1875): t. latissime umbilicata, convexiuscula, anfr. 6 , ultimus antice dilatatus, valde deflexus, apertura perobliqua, obovata (externe rotundata, interne valde angustata), marginibus valde approximatis; d. $15-18$, a. $8 \mathrm{~mm}$. (Gall. ad Agen, German. ad Saalfeld.) Testa gall. alba, unicolor, t. germ. fasciata et minor. 
195. H. obvia Z. ap. IIARTM. (1840): t. mediocriter umbilicata, porcellaueo-candida, obscure fisciata; anfr. ultimus antice non descendens; apertura albolabiata; diam. 16-18, alt. 7-8 mm. (Europa merid.-orientalis; occid. versus in Eur. merid. ad $27^{\circ}$; Ital. max. bor.) 'T. typice depressa, nitida, fascia suprema nigrobrunnea, fasciis siepissime perangustis et inferioribus srepe in maculis dissolutis, anfr. 5, planiusculi, ultimus supra planulatus, infra tumidus, apert. magna, lunato-rotundata, peristomate acito, intus srepius et longe distanter bis albolabiata, marginibus approximatis. Syn. II. neglecta Ifartm. ap. Sturm, H. ericetorum Held. Fig. Hartm. Gast. t. 45. - Variat: usta Held: t. minor, supra sape flammulis brunneis et fasciis pluribus latiusculis vel confluentibus nigro-brumeis.

- var. candicans Z. ap. PFr. (18+1): t. convexa, paullo magis striata, alba, sæpius unicolor, anfr. convexi, ultimus rotundatus. (Eu. max. merid.-orient.) Variat 1) arenosa Z. ap. Rossm. (1838): t. cretacea v. calcarea, alba, unicolor, late umbilicata, spira plus minus elevata ad subconica, anfr. convexi, upert. subcircularis, albolabiata: d. 16, a. 9-11 mm. (Tauria.) Eig. Rossm. f. 510. 2) dejecta Jan (1832): t. alba, cretacea, unicolor vel peranguste fasciata. (Tauria.) Fig. Rossm. f. 520.

196. H. aberrans Mouss. (1863): t. late umbilicata, depressâ, vix striatula, alba, indistincte griseo-marmorata, nitida; anfr. ult. rotundatus, non descendens; apertura "lineato-circularis", fortiter albo-labiata; diam.? (Constantinopolis.) T. rare punctulata, spira subirregularis, anfr. 5, convexiusculi, ult. latior. subtus de medio longitudinis a perforatione distincta devians, angulo dorsali supero rix prolucto, infero columellari vix reflexiusculo. (Mouss.)

197. H. ammonis Scrm. ap. Sтroв. (1857): t. mediocriter umbilicata, convexa, albida, fasciata; anfr. ultimus antice non descendens; apertura rotundata, valide rufescenti- vel violacco-laliatr; diam. 15, a. $9 \mathrm{~mm}$. (Italia bor., Dalmatia.) Differt ah $H$. ericet. spira magis elevata, anfractu ultimo minus angusto, umbilico angustinre, apertura rotundiore et labio valido, rarissime albido; diff. ah $I I$. obvia var: cand. testa minus alba, rarius unicolori, umbilico minus angusto, apertura minus obliqua et labio colorato. - Huc verosimiliter $H$. candida Porro: t. nivea, unicolor, sublevis.

198. H. bathiomphala Crr. ap. Prr. (1848): t. aperte umbilicata, depressa, plerumque concolor albida; anfi. ult. teres, antice sensim descendens; apertura truneato-ovalis, remote et valide bilabiata; diam. 23 , alt. $12 \mathrm{~mm}$. (Italia in Aprutiis.) T. nitida, vix striatula, swpe varie fusco-fasciata, interdum fusco-strigata, fasuia alba peripherica circumulata, anfr. 6, convexiusculi, apert. intus lu- 
fescens, marginibus nimium approximatis. Fig. Bull. mal. ital. 1869. t. 3. f. $6-8$.

199. H. pampelonensis A. Schur. (1855): t. aperte umbilicata, subdepressa, fulvido-alba, plerumque fusco-unifasciata; apert. lunato-rotundata, leviter albo-labiata; diam. 21-22, alt. 10-12 mm. (Hispania.) T. levissime striatula, spira subconoidea v. vix elevata, anfr. $6{ }^{1} / 2$, vix convexiusculi, ult. latior, fulvidus, infra medium obsolete fasciatus, apert. marginibus valde convergentibus. Fig. Mal. B1. 1855. t. 3. f. 4-11. Variat lactea v. cinerascens, fasciata, ad aperturam fulvida; d. 14--16, a. 7-8 $\mathrm{mm}$.

200. H. semipicta Hid. (1870): t. mediocriter umbilicata, depressa, pallide lutescenti-albida, superne maculis pellucidis fuscocorneis profuse variegata et prope peripheriam unifasciata; anfr. ult. convexus, basi subdepressus, antice paullo descendens; apertura subrotundato-lunaris, sublabiata; diam. $9^{1} / 2$, alt. $4 \frac{1}{2} \mathrm{~mm}$. (Hispania.) T. tenuis, subopaca, supra minute striata, sutura impressa, anfr. $5-5_{12}^{1 /}$, convexiusculi, spira convexiuscula, apert. marginibus vix convergentibus. Fig. Journ. Conch. 1871. t. 12. f. 5.

201. H. zapateri HID. (1870): t. mediocriter umbilicata, depressa, cornea, unicolor; anfr. ult. superne obtusissime angulatus, basi convexus, non descendens; apertura exacte lunaris, non labiata; diam. 10, alt. $4^{1 / 3} \mathrm{~mm}$. (Hispania in Belagazar.) T. tenuis, pellucida, subnitida, fere lævigata, sutura impressa, anfr. 51/2, convexiusculi, angusti, ultimus latior, spira convexiuscula, apert. marginibus distantibus. Fig. Journ. Conch. 1871. t. 12. f. 4. - Huc?

\section{it Testa regulariter costulato-striata.}

202. H. stiparum Rossm. (1854); t. supra convexa, subtus turgida, confertim capillaceo-striata; anfr. ult. subangulatus, subdescendens; apertura remote albo-labiata; diam. 20, alt. $11 \mathrm{~mm}$. (Hisp. ad Almeria.) T. cretacea, rarius maculose fusco-fasciata, opaca, anfr. 6, apertura rotundato-lunata, peristoma rectum, acutum. Fig. Rossm. Icon. f. $820-822$.

203. H. adolfi Prr. (1854): t. subconoideo-semiglobosa, spira convexa, confertim costulato-striata, alba, ferrugineo-fuscoque variegata et fasciation maculata; anfr. ult. teres, non descendens; apertura rotundato-lunaris, rufo-albidove labiata; diam. 20-25, alt. 11-14 mm. (Hispania.) T. aperte umbilicata, solidula, anfr. 6, sensim accrescentes, apert. marginibus conniventibus. Syn. $H$. terveri Rossm. 1854. Fig. Rossm. Icon. f. 816-819.

204. H. instabilis Z. ap. PFr. (1842): t. depressa, spira fere plana, regulariter rugoso-striata, solida, albido-lutescens; anfr. 
ult. teres, vix descendens; apert. subcircularis, subtilitur labiata, marginibus convergentibus, approximatis; diam. 14, alt. $7 \mathrm{~mm}$. (Galisia, Transsitx., Tauria.) T. late umbilicata, interdum fuscescens, concolor vel dilute fasciata, anfr. 5 , convexiusculi, apertura vix obliqua, margine superiore æequali altitudine ac anfr. ultimus, imo interdum ac spira. Fig. Rossm. Icon. f. 518.

- var. spimla ZeL. in sched.: t. globoso-depressa, spira convexa v. conoidea, albida, punctis nigris aspersa vel fascia angusta pallide brunnea et punctis brunneis, anfr. ultimi tereti-convexi, ultimus sensim et valide descendens, apertura ovato- vel rotundato-lunaris, obliqua, depressa; d. 14, a. 8-81/2 mm. (Ty anssitr. Dobrudscha.) II. dobrudsche et $H$. transsitvanica Parr. sunt formx intermedir, illa paullo major, hæc minus convexa, anfr. ult. non descendens, apert. obliqua, vix labiata.

- var. grega Mart. (1872): t. depressa, spira convexiuscula, alba vel lutescenti-albida, unicolor vel subtus abrupte fasciata, nitidula, regulariter sed tenue striata, anfi. ult. superne obsolete angulatus, non descenderis, apert. rotundata, vix labiata, parum obliqua; d. 15, a. $7 \mathrm{~mm}$. (Grecia ad Nauplia et Tripoleza.) Fig. Mal. B1. XX. t. 2. f. 1. - H. ephigenice Dub. (e Tauria) ap. Pfr.?

205. F. arenivaga MAB. (1868): t. depressa, spira convexiuscula, conferte striata, albescens v. rufescens; anfractus ult, obsolete angulatus, paullo deflexus; apert. ovato-rotundata, lunata, albo-labiata; diam. 19-20, alt. 9-10 mm. (Gall. mevil.) T. aperte umbilicata, solida, opaca, quandorue maculis et lineis fuscis ornata, apertura oblicqua.

3) Heliomanes Fér.: testa anguste umbilicata (umbiliro vel angusto, subcylindrico, rel superne latiuscuto, sed forte spiratiter coarctato) rel perforata (raro mediocriter umbilicata), globosa $v$. spira turbinata, non $\mathrm{ct}-$ rinata; anfi. ult. teres, convexus; apertura rotundato-lunata, margine columellavi dilatato, patente.

+ Testa lavigata vel irregulariter striata.

206. H. variabilis Dre. (1801): t. anguste umbilicata depresso-turbinata; anfractus ultim. rotundatus, magnus, vix descendens; apertura rotundato-lunat $\Omega$, fusco-labiata; diam. 19, alt. 12 mm. (Regio miditeranca, Britannia.) T. glohoso-subdepressa, spira plus minus turbinata, solida, opaca, lievigata, albiela vel sordide lutescens, fasciis fuseis, sape interruptis, multimole ornata, raro conco- 
lor, anfr. 5-6, convexiusculi, ultimus tumidus, apert. marginibus longe distantibus. Syn. H. virgata Mont. 1803. Fig. Rossm. Icon. f. 356. Variat 1) albita, unicolor, spira paullo elatiore; d. 17, a. 11-12 mm. (Rossm. f. 356 c); 2) parvula, alba, fasciis nonnullis fuscis; d. 10, a. $6 \mathrm{~mm}$. (H. agreabilis Z.); 3) fasciis interruptis moniliformibus fuscis ( $H$. monilifera Mke); 4) minor, albolabiata, fasciis submoniliformibus nigris, d. 12, a. $8 \mathrm{~mm}$. (H. moesta Parr.) \&:c.

207. H. globuloidea Terv. (1839): t. minus anguste umbilicata, subglobosa v. conoideo-depressa; anfr. ult. màgnus, basi convexus, non descendens; apertura lunato-rotundata, albo- v. carneo-labiatum; diam. 20, alt. 14-15 mm. (Algeria, Sicil., Ital. merid., Grceia ad Nauplia.) 'T. alba, unicolor vel multifasciata, interdum lutescens, irregulariter striatula, anfr. 6-7, sat convexi, ult. superne ad suturam planulatus, apert. rarius paullulum ovata marginibus distantibus. Syn. H. arenarum Bourg. 1862. Variat altitudine spiræ: d. 20 , a. 12 ; d. 22 , a. 14 ; d. 21 , a. $17 \mathrm{~mm}$. Forte tantum una extrema præcedentis: forma globosa.

208. H. lauta Lowe (1833): t. submediocriter umbilicata, globoso-depressa; anfr. ult. major, compressus et obtuse subcarinatus, non descendens; apert. subovata v. subrotundata, parum lunata, albido- vel fusco-labiata; diam. 12-14, alt. $7-b \mathbf{m m}$. (Gall. merid-occ., Corsica; teste celeb. Bourg. late distributa circa Mare nigrum.) T. lutescenti-albida, subtus fusculo-variegata et fasciolata, rarius unicolor, lævigata, spira convexa, anfr. 6, convexiusculi, ultimus utrinque compressus, peripheria antice omnino rotundatoconvexus. Syn. H. submaritima Rossm. 1839. Fig. Rossm. Icon. f. 575. Variat major: d. $20-22$, a. $12 \mathrm{~mm}$. (Gall.) - Forte altera extrema forma $H$. variabilis: $t$. depressa.

209. H. proteus (Z. ap. Rossur, 1838): t. anguste umbilicata, depresso-globosa, lævigata, cretacea; anfr. ult. magnus, rotundatus; apert. lunato-subcircularis, leviter labiata; diam. 10-11, alt. $6-7 \mathrm{~mm}$. (Corfu.) T. albida vel nivea, concolor vel sæpius infra maculose fasciata, nitidula, anfr. 6 , convexi, supremi tenuissime regulariter striati, ultimus infra sat tumidus, apertura magna, marginibus distantibus, basali forte arcuato. Syn. H. protea Z. Fig. Rossm. Icon. f. 521. - Variat major, depressior, umbilico paullo latiore $\langle$ H. campestris Z., H. pustulata Nühlf.) - Obs. In Symb. Helic. 1842 et in Mon. Hel. 1848 clariss. Pfeiffer speciem descripsit, quam tantummodo formam $H$. protei existimo. Hæc est $H$. nivea $Z$. ap. Anton 1839: "t. perforata, depresşo-conica, solidula, nitidula, nivea; anfr. 5, vix convexiusculi, ultimus multo latior, descendens; umbilicus angustissimus, non pervius; apertura magna, oblique lunato-ovalis; peristoma subsimplex; diam. $11^{1} / 2$, alt. $7 \mathrm{~mm}$. (Corfu.)" Testa II. protei 
inter confines maris sæpius unicolor, procul a mari divertissime picta esse solet. (Roth.)

210. H. terveri Micir. (1S3): t. perforata vel anguste umbilicata, globoso-depressa; anfr. ult. primo utrinque sat compressus et carinatus, antice rotundatus, descendens; apertura rotundatolunata; diam. 15, alt. $9 \mathrm{~mm}$. (Gall. merid.-orient., ins. Levanzo.) 'T. tota alba, vel alba aut cinerea fasciis fuscis sæpe interruptis ornata, tenniter sat regulariter striata, anfr. $51_{2}-6$, ultimus sat rapide accrescens, subventricosus, apertura latissime lunata, margine columellari fortius quam in precedentibus arenato. Fig. Dup. Ilist. t. 13. f. 5, Journ. Conch. 1869. t. 9. f. 6. - Variat major: d. 18, a. 10-11 mm.

211. H. ambieliana Criare. ap. Palad. (1867): t. angustissime umbilicata, spira parum conoideo-elevata; anfr. ult. non descendens, peripheria subangulatus, subtus convexior; apertura rotundato-lunaris, tenuiter labiata; diam. 13, alt. $7^{1} / 2 \mathrm{~mm}$. (Gallia merid.) T. subirregulariter striata, albido-lutescens, sutura impressa, anfr. 5, subregulariter crescentes, apert. marginibus convergentibus.

212. H. krynicki Axdrz. (1833): t. rimato-perforata (umbilico mediocri spiraliter coaretato), depresso-convexa; anfr. ultimus inflatus, subdeviatus; apert. lunato-subcircularis, albolabiata: diam. 16, alt. $8 \mathrm{~mm}$. (Tamia, Croatia \&o.) T. tenuis, vix strintula, flavescenti-albida vel nivea, fasciis lineisque subnigris (suprema latissima sæpissime præsens) subtus fascidulis intorruptis ornata, raro unicolor, anfr. $3^{1} / 2-6$, convexinsculi, apertura sæepius pertente labiata, peristomate concolore (in plerisque ceteris hujus sectionis peristoma intus obscurum.) Syu. II. babondubii Parr. Fig. Mal. B1. 1871. t. 7. f. $4-7$.

21\%. H. psudenhalia Botra. (1860): t. anguste umbilicata, semiglobosa, cretacea; anfr. ultimus rotundatus, convexus, paullisper descendens; apertura exacte rotundata, parum lunata, valide rosaceo- vel luteolo-labiata; diam. 10-11, alt. $8-9 \mathrm{~mm}$. (Gallia, ins. Chat. d'If.: Bourg., Bordeaux: Gass. ex.; Itatia, Camp. liomana: J. Collin ex.) T. solida, sordide candida unicolor vel rarissime fascia obsoleta interrupta supramediana, lavigata, opaca, spira convexo-turbinata, anfr. 6, convexi, regulariter accrescentes, apertura marginibus intus fuscis approximatis. Fig. Bourg. Chat. d'If., t. $1 \mathrm{f}$. 17-21. - Variat 1) major: d. 15, a. $11 \mathrm{~mm}$. \& d. 16, a. $13 \mathrm{~mm} . ; 2)$ minor: d. 8 , a. $6 \mathrm{~mm}$.

214. H. spadæ CALC. $(1845)$ : t. mediocriter umbilicata, depresso turbinata, subsemiglobosa, calcarea; anfr. ult. teres, non descendens; apertura rotundato-lunaris, sublabiata; diam. 8, alt. 5 
mm. (Italia, in Aprutiis.) T. solida, striatula, anfr. 5, convexiusculi, umbilicus conicus, apert. intus fulvida. Syn. H. destitute Ch. ap. Pfr. 1853, $H$. candida Costa, $H$. ocellus Villa \& $H$. mubila Ch., teste Tiberi. Fig. Rey. Zool. 1859. t. 15, f. 6-10.

215. H. parva PARr.: t. angustissime umbilicata, turbinatodepressa, sordide lutescens, unicolor; anfr. ult. ventricosus, medio obsolete angulatus, antice rotundatus, non descendens; apert. rotundato-lunaris, alloolabiata; diam. 9-10, alt. $61 / 2 \mathrm{~mm}$. (Sardinia) T. nitida, lavigata, spira conoidea, apice subnigra, anfr. 51/2, vix convexiusculi, apertura intus flavida, marginibus longe distantibus. Fig. Chemn. Cab., Hel., t. 113. f. 18, 19.

\section{tf Testa regulariter striata-vel costulato-striata.}

216. H. cretica FḱR. (1819): t. mediocriter et pervie umbilicata, depresso-globosa, rugoso-striata, albida, concolor v. fasciis interruptis varie picta; anfr. ult. vix descendens; apert. subcirculari-lunata, distanter tenue bis vel ter albolabiata; diam. 16, alt. 10-11 mm. (Gracia: Candia, Rhodus, Athenæ.) T. solida, nitidula, anfr. 5-7, lente accrescentes, convexiusculi, ultimus rotundatus, teres; apert. margine columellari fortissime arcuato, superne verticali. Fig. Chemn. Cab., Hel., t. 37. f. 21,22. Variat spira conica: d. $18-19$, a. $20 \mathrm{~mm}$.

217. H. danieli Bounc. (1860): T. globoso-depressa, regulariter costulato-striata, albida, cretacea, efasciata; anfr. ult. rotundatus, non descendens; apertura intus albido-incrassata; diam. 10, alt. $7 \mathrm{~mm}$. (Gall. ad Brest.) T. anguste umbilicata, opaca, anfr. 7, convexi, apertura rotundata, vix lunata, peristomate recto, acuto, marginibus sat approximatis. Fig. Bourg. Malacol. Bret. 1860.

218. H. subrostrata FÉr. (1819): t. mediocriter umbilicata, conoideo-globosa, confertim argute striata, alba, cornea; anfr. ult. non deflexus; apertura labiata; diam. 18, alt. $14 \mathrm{~mm}$. (Hisp.) T. solida, anfr. $6-6 \frac{1}{2}$, convexiusculi, lente accrescentes, ultimus reliquis paullo major, apertura lunato-rotundata, marginibus conniventibus. Fig. Chemn. Cab. Hel., t. 37, f. 15, 16. (Variat minor, interrupte fasciata: Algeria.)

219. H. ebusitana HID. 1869): t. mediocriter umbilicata, convexo-depressa vel depressa, confertissime piloso-striata, cornea, fasciis fuscis et maculis opaco-albis varie picta; anfr. ultimus antice valde deflexus; apertura albo-labiata; diam. 16, ait. $7 \mathrm{~mm}$. (Ins. Ebuso Balearium.) T. umbilico $3 \mathrm{~mm}$. lato, spira obtusissima ant plana, anfr. 6 , vix convexi, ult. basi convexus, prope umbilicum sublævigatus, apert. lunato-circularis, marginibus valde conniventibus, callo tenuissimo subjunctis. Fig. Journ. Conch. 1869, t. 2. f. 2. 
220. H. gyroides Parr. ap. Frr. (1870): t. depressa, spira breviter turbinata, calcarea unicolor, ruguloso-striata; anfr. ult. rotundatus, non descendens; apertura oblique lunaris, leviter labiata; diam. 10, alt. $51 / 2 \mathrm{~mm}$. (Croatia.) T. mediocriter umbilicata, anfr. 6, modice convexi, apertura parum obliqua, marginibus vix convergentibus, basali intus subincrassato, stricto. Syn. H. homolenca Sablj.? ap. Brus. Fig. Nov. Conch. IV. t. 117. f. 16-18.

221. H. luteata Parr. ap. PFr. (1857): t. rimate-perforata, subconoideo-depressa, conferte striata, lutescens unicolor vel albida, fasciis fulvis et fuscis ornata; anfr. ult. basi modice convexus, antice dilatatus, descendens; apert. transverse lunato-ovalis, labiata; diam. 16, alt. $8^{1 / 2}-9 \mathrm{~mm}$. (Lusitania.) T. solidula, anfr. 51/2, planiusculi.

222. H. turbinata $J_{A N}$ (1832): t. perforata, conico-globosa, minute costulato-striata, alba, fasciis et flammulis fuscis variegata; anfr. ultimus inflatus, rotundato-convexus, teres, non descendens; apertura rotundato-lunata, labiata; diam. 11-12, alt. 8-91/2 mm. (Syra, Creta, Sicilia?) T. solidiuscula, anfr. 51/2, convexinsculi, apert. marginibus distantibus. Syn. H. candiota Friw., H. filograna Villa, H. pustulosa Parr., H. cyclostomoides Porro, $H$. pilulu Mouss. Fig. Chemn. Cab. Hel., t. 37. f. 17, 18.

- var. aradasii MLandral. (1842): t. semiobtecte perforata, globoso-conoidea, fortius costulato-striata, albida v. rufescens, unicolor vel sxpius fusco-multifasciata, anfi. ult. magnus, teres; $d$. $81 / 2-101 / 2$, a. $6-7 \mathrm{~mm}$. (Sicit., Ital. in Calabria.) Fig. Ben. Ill. Sie. t. 3, f. 6.

-- var. pellucens Siluttu. ap. PFr. (1848): t. perforata, tenuis, confertissime striata, diaphana, pallide fulvescens, lineis et fasciis saturatioribus, sæpe interruptis, ornata, anfr. ult. basi ventrosus, apertura lunato-circularis, sublabiata; d. $12^{2} / 3$, a. $9 \mathrm{~mm}$. (Corsica.) Fig. Moq.-Tand. Hist. t. 19 f. 24.

223. H. cistorum Morex. (1845): t. anguste umbilicata, convexa, confertim pliculato-striata, cinereo-cornea, albido-marmorata; anfr. ult. depressus, obsolete angulatus, zonula nigricante interrupta ornatus; apertura rotundato-lunaris, vix labiata; diam. 12 , alt. $61 / 2 \mathrm{~mm}$. (Lusitania in prov. Alemtejo.) T. tenuiuscula, anfr. 6, subplani, ad suturam turgiduli, apertura diagonalis. Fig. Chemn. Cab., Hel, t. 128. f. 11-13.

224. H. rufolabris Ben. ap. Pfr. (1856): t. angustissime umbilicata, turbinato-globoso, irregulariter striata, alba; anfr. ult. inflatus, non descendens; apert. rotundato-lunaris, crasse rufulo- 
labiata; diam. 11, alt. $7-7 \frac{1}{2} \mathrm{~mm}$. (Sicilia.) T. solida, anfr. 5, vix convexi, ultimus antice lutescens. "Forma similis. H. aradesii Mandr., sed affinior varietatibus nonnullis $H$. variabilis" (Pfr.)

225. H. pyramidata DRP. (1805): t. angustissime umbilicata, turbinata, sub lente costulato-striata; anfractus lente accrescentes, teretes, ultimus non descendens, basi planiusculus; apertura depressa, late lunaris, labiata; diam. 11, alt. $7 \frac{1}{2} \mathrm{~mm}$. (Regio mediterranca.) T. basi lata, subplana, supra conica vel pyramidata, acuta, albida, unicolor vel varie fusco-fasciata, tæniata, punctulata vel variegata, supra tenuissime sed regulariter costulato-striata, infra $a b$ umbilico radiatim striata, anfr. 6-7, convexi, ultimus medio linea tenui subangulata. Syn. $H$. agnata, arenaria, litoralis, subulosa et spectabilis Z., requieni Jeniss. Fig. Rossm. Icon. f. 349. - Variat 1) major: d. 15, a. $10 \mathrm{~mm} ., 2)$ spira elata, 3) spira depressa.

- var. tarentina PFr.: t. confertim costulata, anfr. ult. medio subangulatus, basi subplanatus. (Sicil.) Syn. H. pyr. v. costulata Rossm. f. 690 .

(H. dormiens Ben.)

4) Jacosta M. TAND.: testa aperte umbilicata (ravissime perforata), compresso-carinata, costulata, spira depressa vel raro breve pyramidata; apertura securiformis, labiata, marginibus acutis, rectis.

\section{† Spira pyramidata.}

226. H. dormiens BeN. (1859): t. semigloboso-pyramidata, costulato-striata; anfractus 6, ultimus medio angulato-carinatus, antice non descendens; diam. 13, alt. $8 \mathrm{~mm}$. (Ins. Maritimo Sicilice.) T. mediocriter umbilicata, albida, inferne rufo-multifasciata; superne maculis strigisque fuscis ornata, anfr. carinati, subplanulati, ult. basi convexus, apert. lunato-angulata, marginibus distantibus. Fig. Ben. Ill. Sic. t. 3. f. 4. - Variat 1) unicolor, albida, interdum subtus obsolete rufo-fasciata; 2) rugulosa: t. albido-rufescens, costulato-rugosa, subtus unicolor, supra maculis distantibus fuscis seriatim dispositis ornata.

227. H. calcarata BEN. (1862): t. subgloboso-pyramidata, costulato-striata; anfr. 6, ultimus compresso-carinatus, non descendens; diam. $7-8$, alt. $4-5 \mathrm{~mm}$. (Malta.) T. mediocriter umbilicata, albida, concolor vel utrinque corneo-fasciata, spira convexa, obtusa, anfr. convexiusculi, carinati, apertura lunato-angulata, vix obliqua, intus remote labiata. Syn. H. schombrii "Scacchi", Pfr. ("Sicilia.") Fig. Ben. Ill. Sic. t. 5, f. 11. 
228. H. sideritis Friw. ap. MAnT. (1871): t. pyramidata, rugosa; anfractus $t^{1} / 2$, ultimus carinatus, antice descendens; diam. 9, alt. (6 mm. (Syra.) T. mediocriter umbilicata, subtus convexa, alba, interdum supra fusco-maculata, infra fusco-bifasciata, carina rugis denticulata, anfr. superiores complanati, antepenultimus gradatim exserti, apertura securiformis, antrorsum albo-labiata, margine supero subhorizontali, basali valde arcuato.

\section{(H. timeana Ben. var.)}

\section{*) Spiva ralde depressa.}

229. H. filimargo Z. ap. Rosssr. (1857): t. perspective umbilicata, depressa, calcarea, raro fasciata, substriata; anfr. convexiusculi; apertura rotundata, remote sublabiata; diam. 14, alt. $6 \mathrm{~mm}$. (Tauria.) T. planiuscula, infra convexa, supra medium filocineta, apertura extus angulata, peristoma rectum, acutum. Fig. Rossm. Icon. f. 358 .

230. H. explanata MüLL. (1774): t. perspective umbilicata, depressissima, albo-lutescens, concolor, tenuissime striata; anfr. subplani, ultimus paullulum sub carina descendens; apert. semirotundato-securiformis, albolabiata; diam. 14-17, alt. 5-6 $\mathrm{mm}$. (Gall. merid., Hisp. merid.) T. solida, opaca, planospira, infra valde convexa, acute carinata, rarius albescens, apertura ad carinam compresse angulata, margine exteriore subhorizontali, interiore forte vel in angulo subrecto curvato, peristoma rectum, acutum. Syn. H. albella Drap. 1801. Fig. Rossm. Icon. f. 539. Variat anfractibus superioribus plus minus exsertis.

- val. depressula Park. ap. Rossm. (1839): t. lenticularis, rugoso-striata, lutescens, medio carinata, utrinque subrqualiter convexa, carina denticulata; d. 12, a. $5 \mathrm{~mm}$. (Gullia al Grasse: Gassies ex., Hisp. merid: Dup.) Ex Oran Africæ cl. Parreyss misit formam majorem (d. 18--20, a. 6-9 mm.) albidam anfractibus ad suturam immersam tumidulis. Fig. Rossm. Ieon. f. 562.

231. H. setubalensis PrR. (1850): t. aperte umbilicata, lenticulari-depressa, fulvido-albida, confertim costulata; anfr. planulati, in carina contigui, ult. paullulum descendens; apertura late lunato-securiformis, albo-labiata; diam. $9-10^{1} / 2$, alt. $31 / 2-3^{2} / 3$ mm. (Lusitania.) 'T'. interdum superne ad suturam maculata, infra fasciata, supra depressa, infra intus tumido-convexa, scrulato-carinata, apertura perolsliqua, marginibus conniventibus, basali paullo reflexo. Syn. H. serrule Mor. 1815. Fig. Rossm. Icon. f. 829. - Huc verbotim pertinet $H$. planissima Pfr. in Proc. Zool. Soc. 1845. p. 72 \& Mon. Hel. I. p. 211 e loco ignoto, tantummodo paullo major: d. $11^{1 / 2}$, a. $41 / 2 \mathrm{~mm}$. 
232. H. homeyeri Donns \& Hers. (1862): t. late umbilicata, depressa, coerulesceuti-albida, fusco-maculata et cingulata, striata; anfr. vix convexiusculi, ultimus descendens; apertura securiformis, labiata; diam. 12, alt. $4 \mathrm{~mm}$. (Ins. Mallorca.) T. supra planiuscula, subtus intusque convexa, carina compressa, crenulata, umbilicus $5 \mathrm{~mm}$. latus, peristoma rectum.

233. H. nyelii Mrtree (1842): t. latiuscule umbilicata, conoideo-depressa, albida, superne maculis fuscis seriatis, subtus plerumque fasciis fuscis ornata, conferte subtiliter costulata; anfr. vix convexiusculi, ultimus subdeflexus; apertura securiformis, labiata; diam. $11^{1} / 2$, alt. $5 \frac{1}{2} \mathrm{~mm}$. (Ins. Mrinorca.) T. suberenulatocarinata, spira brevissime conoidea, interdum pallide lutescenti-cornea, anfr. ult. supra ad carinam late depressus, subtus a peripheria usque convexus, umbilicus $3 \mathrm{~mm}$. latus, apert. perobliqua vix angulata, marg. basali reflexiusculo.

234. H. frater DoHRN \& Heys. (1862): differt a præcedente testa anguste umbilicata (umbilicus ${ }^{3} / 4 \mathrm{~mm}$. latus), anfractu ultimo basi convexo (non ad carinam convexo, medio planiore, circa umbilicum angulato). (Ins. Balear.)

235. H. eremia n. sp: t. plane lentiformis, perdepressa, subtus paullo convexior, utrinque striis costulosis et nodulosis irregularibus rugosa, rufescenti-cornea, annulo niveo circa umbilicum notata; anfr. planulati, ultimus descendens; apertura securiformis; diam. 8, alt. $31 / 2 \mathrm{~mm}$. (Pyrencere altiores.) T. acute carinata, carina pallida, superne paullulum compressa, minutim serrulata, umbilicus- mediocris $\{1 / 4$ diametri requans, conicus, ambitu obtuse carinato, anfractus 5, ad suturam sultumiduli, apertura horizontalis, depressa, vix labiata, margine supero acuto, columellari brevi obliquo, basali subrecto, reflexiusculo, albo. Specimen unicum hujus ut videtur bene distinctr speciei sub nomine $\mathrm{H}$. constrictæ benigne communicavit celeb. Morelet. Aliquo modo præbet formam $H$. nyelii et structuram $H$. nodoso-striata Mouss. (e Gomera.)

236. H. catocyphia BourG. (1860): t. rimato-perforata, supra planulata, omnino albida, cretacea, striata; anfr. planulati, ultimus non descendens; apertura lunata, intus paullulum incrassata, callo dentiformi in pariete munita; diam. 10, alt. $6 \mathrm{~mm}$. (Gallia, ins. "île du chateau d'If" prope Marseille.) T. acute carinata, subtus convexa, anfr. $4^{1} i 2$, sat celeriter accrescentes, ult. magnus, apert. subangulata, in ventre anfr. penultimi tuberculo cretaceo, marg. colum. reflexiusculo, perforationem subobtegente. Fig. Bourg. Malac. terr. chat. d'If, t. 1. f. 1-3. - Opinio celeb. Pfeifferi, hanc testam eadam ac $H$. callosa Anton (1839) e loco ignoto esse, vera videtur. 
237. H. rozeti Mrcrr. (1833): t. anguste umbilicata, lenticularis, utrinque subrqualiter convexa, costulato-striata, sordide albida, fusco-marmorata et interrupte fasciata; anfr. convexiusculi, ultimus compressus, non descendens; apertura securiformis, albolabiata; diam. 12, alt. $6 \mathrm{~mm}$. (Sicilia, Mrorea.) T. carinata apertura, extus angulata, margine columellari declivi. Syn. H. striatula Lin., Carocolla limbata Phil. 1836, H. amanda Rossm. 1839. Fig. Rossm. Icon. f. 449. - Variat depressior, umbilico latiore (H. boissyi Terv.)

- var. usticensis Calc. (1840): differt anfractibus planioribus, ultimo circa umbilicum perangustum compresso, apertura depressiore, margine columellari subverticali; d. $14 \frac{1}{2}$, a. $7 \mathrm{~mm}$.

238. H. tineana BeN. (1862): t. pervio-umbilicata, depressa, subtiliter costulata, sordide allsa, concolor vel rufo-fasciata; anfr. planiusculi, ultimus rotundatus, vix descendens; apertura ovatorotundata, rufo-labiata; diam. $12^{1} / 2$, alt. $5^{1 / 2} \mathrm{~mm}$. (Sicilia.) T. spira subplanata, anfr. ultimus obtuse angulata, subcarinata, carina ad aperturam evanida, basi convexus, apert. antice rotundata, minime angulata, vix obliqua, marginibus conniventibus. Fig. Ben. Ill. Sic. t. 4. f. 24 .

- var. kobeltiana mh.: t. anguste umbilicata, globoso-depressa, spira conoidea, obtusa, rufescenti-brumnea, regione umbilicali lutescenti-cormea, zona mediana et sutura niveæ, apertura pone marginem tenue albo-labiata; d. 11 , a. $5 \frac{1}{2} \mathrm{~mm}$. - Amiciss. Kobelt spec. sub nom. "H. tinei Ben." communicavit.

239. H. rugosa Chexs. (1786): t. sat late umbilicata, depressa, costato-plicata, albida, vel ferrugineo-albida, subtus rufofasciolata; apertura lunato-rotundata, rufescenti labiata; diam. 11-44, alt. 5-6 mm. (Italia infer., Sicitia.) T. supra subplana vel spira paullisper elevata, subtus convexa, carina filiformi crenulata alba cincta, apertura extus angulata, marginibus subapproximatis. Syn. H. corrugata Gmel. 1790, H. gargotta Phil. 1836, Rossm. 1839. Fig. Rossm. Icon. f. 357.

240. H. spratti PFr. (1846): t. perspective umbilicata, depressa, spira prominula, confertim costulata, sordide alba vel fusca, saturatius fasciata, supra spiraliter canaliculata; anfr. elato-gibbosuli, ultimus non descendens; apertura coarctato-securiformis, albolabiata; diam. $8-9$, alt. $3^{1} \frac{1}{2}-4 \mathrm{~mm}$. (ILalta.) T. subtus convexa, circa umbilicum altior, subangulata, carina utrinque compressa, serrulata, superne suturam marginans, apertura acute angulata, parvula, non obliqua, marginibus conniventibus. Fig. Chemn. Cal). Hel. t. 23. f. 9-11. 
- var. schembriana BEN. (1862): t. supra planulata, concolor albida vel ferruginea; d. $11-12$, a. $4-4^{1} / 2 \mathrm{~mm}$. - Fig. Ben. IIl. Sic. t. 4. f. 26.

241. H. montserratensis Hrd. (1870): t. anguste umbilicata, depressa, supra convexiuscula, albida, supra carinam sæpe fusco interrupte unifasciata, pliciforme costulata; anfr. planiusculi, ultimus deflexus; apertura rotundato-lunaris, late albo-labiatum; diam. 13, alt. $7 \mathrm{~mm}$. (Hispania, Montserrat.) T. costulis pliciformibus, irregularibus, subundulosis, ad carinam validioribus confertim sculpta, albida vel sordide lutescenti-grisea, anfr. $5^{1 / 2}$, ultimus planatus, infra valde convexus, umbilicus $1 / 6$ diametri vix æquans, margine columellari reflexiusculo, subdilatato. Fig. Journ. Conch. 1871. t. 19, f. 6 .

242. H. cardonæ Hid. (1867): t. mediocriter umbilicata, lenticularis, fusco-cornea, interdum maculis minimis fulvis sparsis vel ad suturam alternatim dispositis, sub lente striis longitudinalibus brevissime ciliatis ornata; anfr. planiusculi, ultimus descendens; apertura lunaris, ad carinam angulata; diam. 10, alt. $4 \mathrm{~mm}$. (Ins. Minorca.) T. tenuis, supra convexiuscula, subtus convexa, carina acuta erenata, peristoma album, rectum, marginibus conniventibus, basali intus sublabiato.

5) Striatella: Testa anguste umbilicata, conoideo-depressa, striata vel costulata, non carinata, sed anfr. ult. scepe obtuse carinato; apertura rotundato-lunaris.

广 Anfractus ultimus rotundatus vel superne obsolete obtuse angulatus.

243. H. apicina LAM. (1822): t. aperte umbilicata, depressa, spira planulata, ruguloso-costulata, hispidula; apertura lunatorotundata, infra dilatata, vix labiata, peristomate recto; diam.' 7, alt. $4 \mathrm{~mm}$. (Gall., Lusit., Malavia, Dalm., Italia, Hisp. ad Gibraltar, Ins. Balear., Sicilia; Corfu?) T. albida vel griseola, opaca, apice nitido, ad suturan anfractuum superiorum corneo-variegata et făsciis destituta, subtus turgida, anfr. 5, primi angusti, convexi, ultimus supra angustus sed antice subtus dilatatus, tumidus, peripheria rotundus, apertura (in $\mathrm{H}$. cand. latior quam alta, margine basali strictiusculo, in H. striata lat. et alt. æqualis, marg. basali arcuato) altior quam lata, margine basali fortissime arcuata. Syn. $H$. cenisia Ch. 1837. Fig. Rossm. Icon. f. 352. - Variat: zonata M. T., t. infra fasciis angustis (1) paucis. 
- var. remieni M. TAND. (1855): t. spira elevata, convexi v. subconica, umbilico angustiore; d. 7, a. 5. mm. (Corsica: M. 'l., Sicilia ad Catania: Poulsen ex., Gall. ad Toulouse: St. Sim. ex.)

- var. ramburi Mab. (1868): t. subconvexo-depressa, aufr. primi lente, ceteri rapide accrescentes, umbilieus minus apertus, peristoma album incrassatum; d. $9-10$, a. $3-4 \mathrm{~mm}$. (Gallia et prope 'Theodosia 'T'anvioc.)

244. H. striata MüLL. (1774): t. pervie umbilicata, globososubconoidea, ruguloso-costata, glabra; apertura lunato-subcircularis, tenuissime albo-labiata, marg. colum. reflexiusculo; diam. 71/2, alt. $4^{1 / 2}-5 \mathrm{~mm}$. (German., Austria, Bohem., Tirolia, Transsilv., Gallia bor.-occidl.) T. alba vel flavesenti-albida, concolor vel sæpius fasciis strigisque subnigris ornata, solida, costis inrequalibus, sæe bifidis exsculpta, anfr. 5, convexi, supremi lente, ultimi celeriter accrescentes, ultimus multo major, rotundatus, infra tumidus, sutura subcanaliculata, peristoma marginibus subapproximatis. Animal sagittis duabus brevioribus munitum. Syn. H. costulata Z. ap. C. l'fr. 1828, $H$. intersecte L. Pfr. 1853, $H$. narbonensis liequ. teste M. 'T. Fig. Rossm. Icon. f. 533. Variat 1) major: d, 9-10, a, 5-61/2 mm.; 2) minor: d. 5, a. $3^{1 / 2} \mathrm{~mm}$. (Tirol.: II. candidula var, Gr. ex.; Würtemb.). (Foss.)

- var. nilssonianu Beck (1837): t. apice depressa, anfractibus convexioribus, fasciis obsoletis, plerumque nullis; d. $81 / 2$, a. $6 \mathrm{~mm}$. (Succia, Oeland.) Syn. H, ericetorum Nilss. 1822.

245. H. candidula STrud. (1820): t. angustius umbilicati, globoso-depressa, levigata vel minute striata, glabra; apertura inferne depresse rotundato-lunaris, valide albo-labiata, margine basali et colum. late patulis; diam. 8-9, alt. 5-51/2 mm. (Germ. med. \& merid., Gall, Belgia, Tiroliu, Helv., Lusitania, Ital. bor., Carmiol. ad Laibach, Croatia, Rossia ad Kursh, Tauria.) T. nitidula, solida, candida, cretacea usque ad labium aperturæ, unicolor vel fascia ma supramediana et infra treniata vel punctulata, aufr. 5, convexiusculi, lente accrescentes, ultimus magnus, rotundatus, teres, raro supra planiusculus et infra convexior, spira convexa vol etiam subconica, umbilicus superne latiuseulus, spiraliter angustatus, peristoma tenue, cornoum, margine basali rectiusculo, intus dentiforme calloso, et columellari brevi subhorizontali. Animal sagitta unica longa præditum. Syn. H. unifasciata P'oir. 1801. Fig. Rossm. Icon. f. 350. Variat 1) major: d. 10-11, a. 6-7 mm. (H. gratiosa Stud. Rossm. f. 360 b.); "2) minor: (l. $4^{1 / 2}-5$, a. $4^{1 / 2}-4$ mm.

alpicola S'тав. (186t): t. minor, cretacea, globoso-depressa, temuiuscula, fasciis angustis, pallidis, interruptis, evanescentibus, apertura magis rotundata; d. $6^{1 / 2}$, a. $4^{1 / 2} \mathrm{~mm}$. 
- thymorum AlT. (1812): t. turbinato-depressa, obtusa, plus minus striata, porcellanea vel (et præcipue subtus) lutescens, sæpius fascia subcastanea supramediana usque ad apicem producta et infra tæniis nonuullis ornata, anfr. ult. supra planulatus, superne obtuse angulatus, infra convexus; apertura depresse rotundato-lunaris, valide albo-labiata, labio in extremitatibus sinuato. - Syn. H. unifasciata et $H$. candidula persæpe in collect. Huc $H$. thym. var. atrate Kilett et $H$. unif. var. albocinctella Colb.: t. utrinque obseura (fasciis confluentibus) linea alba mediana.

- var. spirilla WesterL. (1875): t. anguste umbilicata, depressa, costulato-striata, spira acutiuscula, prominula, lutescens, sæpius fascia brumea súpramediana, anfr. $41 / 2-5$, ultimus latior, ad aperturam dilatatus, supra planulatus, superne obtuse carinatus, infra convexus, apertura supra et infra angulata, securiformis, valde et late 2-3 labiata, margine colum. brevi cum basali angulum subrectum formante; d. $4^{2} / 3$, a. vix $3 \mathrm{~mm}$. (Gall. merid., Sicilia.) Forma, forte species, H. rugosiusculam in memoriam revocans.

- var. Tumuluta KRYN. (1833): t. striolata, superne angulata, alba, concolor vel fascia et lineis fuscis ornata, spira paullo elevatiore, umbilico angustiore, anfr. 6, rotundati; apertura compresso-lunata intus labiata, labio in extremitatibus sinuato, peristomate patulo. (Odessa.)

246. H. rugosiuscula Micir. (1831): t. mediocriter umbilicata, turbinato-depressa, regulariter confertim costulata, glabra; apertura rotundato-lunaris, valide albo-labiata, margine colum. vix reflexiusculo; diam. 6, alt. $4 \mathrm{~mm}$. (Gallia max. merid.) T. subglobosa, trochiformis vel subdepressa, subtus convexa, utrinque eleganter costulata, costulis teretibus, grisea vel griseo-lutescens, unicolor vel sæpius obscure fasciata, anfr. 5-6, convexi, lente accrescentes vel regulares, ultimus ad initium superne obsolete obtuse angulatus, antice paullo descendens, apertura margine basali arcuatulo.

247. H. paladilhi Boung. (1866): t. subpervio-umbilicata, globoso-depressa, valide irregulariterque rugoso-costata, glabra; apertura lunato-subrotundata, vix labiata, margine colum. vix dilatato; diam. $7-8$, alt. $4 \mathrm{~mm}$. (Gallia, ad Montpellier.) T. albida, fascia una interrupta fuscula supra peripheriam notata, spira convexa, anfr. 5-6, convexiusculi, lente accrescentes, ultimus (in junioribus angulatus) antice vix descendens, sutura impressa, apertura intus non incrassata, margine basali nodice arcuato. Differt ab $\mathrm{H}$. striata forma magis depressa, anfr. lentius accrescentibus, costulis 
validioribus, insequalibus, minus confertis \&e. (Pfr.), al) H. rugosiuseula umbilico magis aperto, costulis et peristomate tenui (Dubr.)

248. H. caroli Dohrn \& Heys. (1862): t. angustissime umbilicata, depresse globosa, supra rugoso-costulata, glabra; apertura perobliqua, lunato-ovalis, albo-labiata, margine colum. breviter expanso; diam. 9, alt. $6 \mathrm{~mm}$. (Ins. Baleav.) T. corneoalbida, castaneo-fasciata, spira obtusissima, sutura impressa, anfr. 51/2, convexiusculi, lente acerescentes, ultimus rotundatus, antice vix descendens, peristoma rectum, marginibus disjunctis. (D. \& H.)

\section{ti Anfractus ultimus sempcr medio plus mimus angu- latus vel carinatus.}

*) Testa omnino glabra; apertura labiata.

a) Destrib. geogr.: Europa merid.orientalis.

249. H. profuga A. Scrm. (1854): t. mediocriter umbilicata, subconoidea, confertim et regulariter costulato-striata; anfr. convexi, ultimus suplo latior, tumidus, obsolete angulatus; apert. parvula, lunato-rotundata, rufo-labiata; diam. 10, alt. 6-7 $\mathrm{mm}$. (Regio meditervanea.) T. albida vel lutescenti-grisea, unicolor vel varie fusco fasciata (sæpius fascia una lata supramediana et infra fasciis pluribus angustis), angulo semper albido, anfr. sensim accrescentes, sed ult. magnus, parum descendens, apertura interdum subovata, marginibus æque arcuatis, labio sæepius rufo-luteo, raro albo, marg. colum. breviter patente. Syn. H. striata Dr., Lam. pr. p., H. caperata Pfr. Mon. 1, III. pr. p., H. fasciolata M. Tand. 1855. Fig. Rossm. Icon. f. 354 b. - Variat 1) major: d. 15, a. $11 \mathrm{~mm}$., t. sericina, unicolor, fulvida, vel leviter fasciata, obsoletissime angulata (Ital. bor.); 2) ctrusca Iss.: t. turbinato-depressa, acute angulata v. subearinata, subtus convexior, d. 10, a. 5-51/2 mm. (Ital. sup., Spalato); 3) late umbilicata, d. 11, alt. $7^{1 / 2}, u_{\mathrm{mb}}$. diam. fere $3 \mathrm{~mm}$. (Spalato: Kistr. ex.) Var. major formæ cuidam II. variab. simillima, sculptura excepta.

(250.) H. meridionalis PArr. ap. Mouss. (1859), "differt ab II. profuga Schm. forma planiore, striis anfractus ultimi rudibus et umbilico amplo" M. (Dalm.: Rossm., et insula Carfu.) syn. II striata var. Rossm., H. submerictionalis Bourg.? Junior $=$ II. striata v. jonica Mouss. Fig. Riossm. lcon. f. $35 t$ e.

(251.) H. variegata Furw. ap, Mouss. (1859) "differt ab II. profuga Schm. spira magis conica, anfractibus minus convexis, striis levibus, in specim. albis et calcareis evanescentibus, et colore albo, non griseo, maculis et fasciis obscuris variegato. (Epirus.) Var. pustulosa Parr. ap. Mlouss. (1863): solidior, magis lævigata, sub striata, lineis et fasciis indistinctis, pallide flavescens, labio tenui albo. (Constantinopolis.) 
252. H. subprofuga STAB. (186t)?: t. peranguste umbilicata, subglobosa vel globoso-depressa, regulariter striata; anfr. convexiusculi, ult. magis tumidus, obsolete angulatus; apert. major, lunato-circularis, pertenue albolabiata; diam. $9-91 / 2$, alt. $6-7$ mm. (Italia ad Napoli: Coll. ex., Avellino ete. Stab.) T. griseo albida, maculis et fasciis abruptis cinereis et pallide brunneis variegata, angulo antice evanescente albida, apert. $5 \mathrm{~mm}$. lata, $4 \frac{1}{2} \mathrm{~mm}$. alta, margine columell, reflexo.

253. H. meda Porro (1840): t. vix rimata, supra globosa, subtus planiuscula, strigis crebris ab infera perforatione irradiantibus; anfr. convexiusculi, ultimus angulatus; apert. irregulariter subquadrata; diam. 10-12, alt. 6-8 mm. (Sardinia, Sicitia, IIalta, Italia in Calabria.) T. albida, aliquando subferruginea sed strigis albis, unicolor vel maculis et fasciis brunneis picta, anfr. 5 , regulariter accrescentes, ultimus subtus ad initium sat compressus, ad aperturam sat tumidus, apertura intus labio angusto rufescenti munita. Syn. H. subclausa Rossm.

\section{B) Destrib. geogr:: Europa occidentalis.}

254. H. caperata MoNт. (1803): t. mediocriter umbilicata, depresso-subglobosa, confertim striata; anfr. 6, convexiusculi, lente accrescentes, ultimus supra visus vix latior, infra extus depressus; apert. obliqua late lunata, candido-labiata, labio insertionem marginis colum. non attingente; diam. $8-11$, alt. $42 / 3-6 \mathrm{~mm}$. (Britan., Gall., Hisp., Lusitan.) T. sordide albida, fasciis corneis, sæpe maculosis vel strigatim interruptis ornata, anfr. ult. angulatus, angulo sæpissime superne fascia fusca terminato, a sutura lente declivus, infra parum convexior, subcompressus. Syn. $H$. striata Drp. pr. p., $H$. cremulata Dillw., H. fasciolata Drouët, $H$. intersecta Anct. mult.pr.p. Fig. Rossm. Icon. f. 831.

255. H. madritensis RAMB. (1868); t. perforata, depressoglobosa vel globoso-conoidea, dense costulata; anfr. 5, convexiusculi, ultimus citius accrescens, basi subturgidus; apertura sat magna, rotundato-lunata, labiata; diam. 9, alt, $6 \mathrm{~mm}$. (Hisp. circa Madrid.) T. albida vel cinerea, supra fasciis 2 macularibus fuscis, interdum obsoletis, ornata, anfr. ult. angulatus, antice parum deflexus, peristoma labro lato extenso. Fig. Journ. Conch. 1869, t. 9. f. 4.

256. H. diniensis Raмв. (1868): t. umbilicata, depressa, tenuissime striato-costulata; anfr. $51 / 2$, parum elevati, ult. major, antice paullo angustatus; apertura ovali-rotundata, intus angustata, profunde albo-labiata; diam. 12, alt. $6 \mathrm{~mm}$. (Gallia, prope Digne.) T. primum anguste dehine latius umbilicata, albida v. cine- 
yea, lineis et fasciis fuscis plerumque ornata, infra convexa et extus subito decliva, anfr. paullatim accrescentes, peristoma rectum, antice subdeflexum et valide devinm, marginibus approximatis, columellari ad insertionem curvo, dilatato, reflexiusculo. "Species magnitudine pervariabilis." Fig. Jonru. Conch. 1869 t. 9. f. 2.

257. H. reboudiana Boung. (1863): "t. umbilico angusto, sæpe perforatione, anfractibus parum convexis costulato-striatis, colore albo, fascia obscura supramediana, vel obscure brunneo-griseo, supra maculis, infra fasciis variegato": Mouss. (Hispania merill.) Nec specimina nec descriptionem originariam in Bourg. Malac. $\mathrm{Alg}$. I. 212. (t. 21. fig. 19-30) vidi.

258. H. intersecta Mich. (1831): t. sat anguste umbilicata, globoso-depressa, utrinque convexa, dense striato-costulata; anfr. 5 -6, subplani, lente accrescentes, ultimus paullo major, obtusissime subcarinatus; apert. perobliqua rotundata, lunata, fulvorufescente labiata; diam. 8, alt. $5 \frac{1}{2}-6 \mathrm{~mm}$. (Gallia merid. \& occid., Hisp. bor.) T. opaca, albido-grisea, nigro-purpurascens, interrupte fulvo vel rufo-fasciata, toniata, maculata vel etiam punctata, raro unicolor, anfr. ult. antice breve descendens, subtus ad peripheriam subito descendens (extus convexus), apert. raro albolabiata. Syn. H. ignota Mab. 1865. Fig. I)up. Ilist. t. 13. f. 1.

259. H. codia Bourg. (1859): t. anguste umbilicata, depressoglobosula, eleganter costulata; anfr. 6, planiusculi, regulariter accrescentes, ultimus rotundatus, antice abrupte descendens; apertura perobliqua, lunato-rotundata, albido-incrassata; diam. 11-12, alt. 7-9 mm. (Lusitania merid.) T. exacte convexa, luteolo-cornea (vel lutescenti-rufescens), lineolis corneo-fuscis eleganter interruptis spiraliter ornata, anfr. ult. infra rotundato-convexus, ad initium obtusissime angulatus, peristoma marginibus approximatis. Syn. H. intersecto Morel. 1845 (ex. sp. orig.) Fig. Rev. Zool. 1859; t. 20. f. 10-13.

260. H. barcinensis Boura. (1864): t. anguste umbilicata, globoso-depressa, utrinque convexa, iñequaliter costulata vel plicatula; anfr. $5 \frac{1}{2}$, convexi, ultimus rapidior, parum deflexus; apertura obliqua, lunato-rotundata, albo-labiata; diam. $10^{1} / 3$, alt. ( mm. (Hisp. prope urbem Miranda.) T. albido-cinerea vel cincreorufescens, fusco aut rufo alboque fasciata et maculata, infra sublievis, medio subcarinata, apertura elata, marginibus approximatis. Syn. $H$. miranda Ramb. 1868, nec Lowe, H. iberica Ramb. 1869. Fig. Journ. Conch, 1869. t. 9. f. 5 .

261. H. gigaxi Cu. ap. Prr. (1850): t. arcuato-rimata (Pfr.), perforata, depressa, confertim striata, subtus convexa; anfr. 5 , 
planiusculi, ultimus latior, ad suturam planulatus, non descendens; apertura lunato-subcircularis, valide labiata; diam. 9, alt. $4_{13}^{2 /} \mathrm{mm}$. (Insitania, Gallia, Brilann., Belgia.) T. opaca, albida vel pallide cornea, subunicolor vel fusco marmorata et interrupte fasciata, spira vix vel paullo turbinato-elevata, anfr. ultimus ad initusn obtuse angulatus, subtus convexus, apertura remote labiata, margine dextro sursum fornicato.

\section{y) Destrib. geogr.: Hispania.}

262. H. derogata Rossur. (1853): t. aperte umbilicata, perdepressa, minute striata; anfractus ultimus depressus, obtuse angulatus, non descendens; diam. 9, alt. $4 \mathrm{~mm}$. (Hisp. bor. et media.) T. nitida, superne albida, peripheria et basi castaneo-fasciata, spira vix convexa, anfr. 5, lente acerescentes, depressi, ult. basi convexior, umbilicus conicus, 1/, diametri fere eqquans, apertura obliqua, oblique rotundato-lunaris, albolabiata, marginibus subeonvergentibus, columellari arcuato-declivi, superne reflexiusculo. Fig. Rossm. Icon. f. 833.

263. H. murcica Gurrao ap. Scrm. (1854): t. umbilicata, sublenticulari-depressa, confertim et tenuiter costulato-striata; anfractus ultimus angulatus, non descendens; diam. 8, alt. $4 \mathrm{~mm}$. (Hisp. prov. Murcia.) T. haud nitens, albida, superne corneo-macuInsa, basi varie corneo-fasciata, spira brevissime conoidea, anfr. 5, convexiusculi, lente accrescentes, ultimus basi parum convexus, umbilicus conicus, $1 / 4$ diametri fere æequans, apertura obliqua, rotundato-lunaris, vix labiata; marginibus subconniventibus, columellari declivi, superne reflexiusculo, Syn. $H$. derogata var. angulata Rossm. (185t). Fig. Rossm. Icon. f. 834 .

264. H. penchinati Bourg. (1868): t. sat pervie umbilicata, valde depressa et valde striata; anfractus ultimus leviter compressus, carinatus, antice declivis et subito valde descendens; diam. $61 / 2$, alt. $3 \frac{1}{2} \mathrm{~mm}$. (Hisp. circa Barcelona.) T. fere semper limo rubello inquinata, supra corneo-castanea ac flammulis albidulis passim aspersa, subtus albidula ac zonulis. nigrescentibus, spira parum convexa, anfr. $5 \frac{1}{2}$, convexiusculi, lente crescentes, ultimus parum major, supra convexiusculus, subtus rotundatus, apertura perobliqua, fere rotundata, parum lunata, marginibus approximatis, columellari vix reflexiusculo. Fig. Bourg. Moll. nouv. \&c. t. 42. f. 7-11.

**) Testa pubescens, apertura clabiata.

265. H. conspurcata DrP. (1801): t. anguste umbilicata, capillaceo-costulata, pilis elongatis numerosis fragilibus et caducis obsita; diam. 6, alt. $3 \mathrm{~mm}$. (Gallia, Lusit., Dalm., Sardin., Sirilin, Ital.) T. depressa, supra vix convexiuscula, subtus convexa, subangulata, cinereo-cornea, minutim variegata, minutissime et irre- 
gulariter costulato-striata, anfr. $5-6$, depressi, paullatim acerescentes, apert. rotundato-lunaris, peristomate recto, acuto, simplici.. Syn. $H$. vadiolate Jan, H. sardiniensis Porro. Fig. Rossm. Icon. 35I. - Variat 1) minor, 2) $f_{\alpha}$ sciata: infra fasciis concentricis (var. draparnaudia M. 'T.).

266. H. vestita RAMB. (1868): t. latius umbilicata, striata, densissime et brevissime pubescens; diam. 5-6, alt. $3 \mathrm{~min}$. (Gallia merid., Hisp., Corsica.) T. tenuis, depressa, spira parum elevata, subangulata, cinerea vel cinereo-subrufescens, supra variegata, tenuiter et irregulariter costulata, anfr. $4 \frac{1}{2}$, lente accrescentes, ultimus parum deflexus, apertura rotundata, sublunata, margine columellari producto.

6) Turricula BecK: testa conica rel trochiformis, carinata, (exc. N:o 26\%) srepe costulata, angustissime umbilicata; apertura depressa, late lunaris vel anguluta.

\section{+ Testa ccarinata.}

267. H. contermina Siutre. ap. PFr. (1848): t. perforata, conico-globosa, confertim plicosula; anfr. 6, perconvexi; apertura lunaris, sublabiata; diam. 6, alt. fere $6 \mathrm{~mm}$. (Corsica.) T. sordide alba, corneo-marmorata, spira conica, anfr. regulariter accrescentes, ult. basi convexiusculus, apert. margine colum. superne subdilatato. - H. psammoica Mor. 1851 e Algeria ("t. perforata, conoidea, pallide grisea, rubigineo-fasciata vel punctata, anfr. 7, convexi, apertura (lepresso lunaris \&c. diam. 8, alt. $9 \mathrm{~mm} . "$ ) species eadem, teste celeb. Pfr. Fig. Journ. Conch. 1851. t. 9. f. 11.

\section{tt Testa carinata.}

*) Testa basi convexiuscula vel subplana, peristoma marginibus disjunctis.

a) Testa altion quam basi lata, trochiformis.

268. H. elata FAUre-Bı́. ap. Fér. (1819): t. conica, albida, costulata, basi carina vix crenulata circumdata; anfr. carinati, exserti; apertura sublabiata; diam. $7-8$, alt. $9 \mathrm{~mm}$. (Sicilia.) T. perforata, costulata, albida, anfr. 9, plani, exserti, crenulato-carinati, apertura securiformis, peristoma rectum, acutum. Fig. Rossm. Icon. f. 344 . Ben. Ill. Sic. t. 5. f. 16. Variat major: d. $9^{1 / 2}$, a. $10 \mathrm{~mm}$.

- var. dilatata BEN. (1862): t. concavo-conica, spira moderatim elevata, basi dilatata, anfr. exsertioribus, apertura depressa, 
extus canali, angulata; diam. $9-10^{1} / 2$, alt. $7-9 \mathrm{~mm}$ : - Fig. Ben. Ill. Sic. t. 5. f. 16.

269. H. sequentina BEN. (1862): t. coarctato-conica, albida, striata, basi carina crenulata cincta; anfr. carinati, non exserti; apertura labiata; diam. 7, alt. $S-\mathrm{S}_{1 / 2} \mathrm{~mm}$. (Sicitia.) T. perforata, anfr. 9-10, supremi vix convexiusculi, læevigati, reliqui plani, marginati, apertura angulato-depressa, peristoma acutum. Syn.? H. py ramis Phil. 1836. Fig. Ben. Ill. Sic. t. 5. f. 18.

270. H. caroni Desh. (1830): t. turrito-conica, lutescens, fusco-maculata, plicata, basi carina grosse serrata cincta; anfr. carinati, exșerti, apertura valide labiata; diam. 8, alt. $11 \mathrm{~mm}$. (Sicitia.) T. perforata, anfr. 10-11, plani, serrato-carinati, apert.depressa, securiformis, peristomate recto, acuto, remote labiato. Syn. H. turrita Phil. 1844, H. elata Cantr. 1840. Fig. Rossm. Icon. f. 343. Ben. Ill, Sic. t, 5 f. 14.

\section{ß) Testa altitudine et latidudine basali aqualis vel la- tior, conica.}

271. H. trochoides PoIr. (1789): t. globoso-conica, irregulariter substriata; anfr. 6, convexiusculi, sutura distincte filomarginata; apertura late lunaris, vix angulata, labiata; diam. 7, alt. $6^{2} / 3 \mathrm{~mm}$. (Regio mediterranea.) T. perforata, solida, opaca, cretacea, albida, unicolor vel fasciata, anfr. ultimus convexus, inferne carina filiformi cinctus, infra ad aperturam præsertim convexiusculus, radiato-striatulus. Syn. $H$. conica Drp. 1801, Helicella solarium Risso 1826? Fig. Rossm. Icon. f. 347.

- var. sulculata JAN ap. Rossm. (1837): t. obsolete carinata, non filocincta, costulato-striata, plerumque fusco maculata, anfr. vix convexiusculi; d. 7, a. $7 \mathrm{~mm}$. (Sicitia, IIalta, Italia etc.) Syn. H. rugosiuscula Mich. 1831, H. rugosa Arad. Fig. Rossm. Icon. f. 348 .

- var. pumilio PrR. (1842): t. paullo latius umbilicata, costulato-striata, carina crenulata.

- var. pyramidella $\mathrm{J}_{\mathrm{AN}}$ (1832): t. trochiformis, umbilicata, striata, obtuse carinata, alba, maculis corneis diaphanis picta. (Ital. bor.)

- var. turvitella PARr. ap. Pfr. (1870): t. trochiformis, perforata, striata, fascia fusca supra carinam et plerumque secunda basali, sutura distincte filomarginata, anfr. $6 \frac{1}{2}$, convexi, uit. subacute filocarinatus; d. 7, a. $6 \mathrm{~mm}$. (Dalm.) Fig. Pfr. Nov. conch. t. 117. f. 22-24. - Var. remissa Parr. est forma minor, anfr.6, supra 
maculis oblongis et striis brunneis transverse decoratis; d. 4 , a. $3^{1 / 2}$ mm. (Ins. Lesina.)

- var. verticillata PARr. ap. PFr. (1870): t. conica, perforata, confertim plicata, alba, sutura funiculato-marginata, anfr. 6, convexi, carina funiformi, sulco a convexitate separata, apert. subangulato-lunaris; d. 5, a. 5-6 mm. (Rhodus: Parr. t. Pfr., Italia: Parr. ex.) Fig. Pfr. Nov, conch. t. 117. f. 19-21.

- var. vidua m.: t. umbilicata, conica, costulato-striata, sordide albida, unicolor, anfr. 7, cylindracei-convexi, omnino rotundati et ecarinati, sutura profundissima separati, apert. subcircularis, vix lunulata, margine columellari intus subincrassato; d. 8, a. $8^{1 / 2} \mathrm{~mm}$. (Spezzia.) Specimen unicum a clar. E. v. Martens acceptum. Monstrositas?

272. H. syrensis PFr. (1846): t. depresso-conica, costulata; anfr. 5, exserti, subplanulati; apertura rotundato-lunaris; diam. 8, alt. $5 \mathrm{~mm}$. (Ins. Syra.) T. anguste umbilicata, sordide albida, fusco vel spadiceo marmorata, anfr. ult. basi convexiusculus, peristoma intus albido-labiatum. Fig. Chemn. Cab. Hel. t. 23, f. 22, 23.

273. H. terrestris PenN. (1777): t. conico-tectiformis, capillaceo-costulata; anfr. plani, contabulati; apertura securiformis; diam. 9-10, alt. $7-8 \mathrm{~mm}$. (Regio mediterranea.) T. angustissime umbilicata, solida, opaca, alba vel corneo-fusca, interdum unifasciata vel maculata, apice acuta, infra planiuscula, basi acute carinata, anfr. 6-7, carinati, apertura acute angulata, intus albo-labiata. Syn. H. elegans Gmel. 1788. Fig. Rossm. Icon. f. 345. - Variat 1) perdepressa, sæpius griseo-rufescens, unicolor; d. 9, a. $5 \mathrm{~mm}$. (H. trochilus Poir. 1789, H. scitula Jan 1832); 2) major: d. 12-13, a. 5-7 mm.

274. H. apiculus Rossm. ap. PFr. (1848): t. trochiformis, plicata; anfr. vix convexiusculi; apertura oblique angulato-lunaris, valide labiata; diam. 9, alt. $6 \mathrm{~mm}$. (Ins. Lampedusa.) T. vix perforata, carinata, alba, fusco marmorata vel flammulata, anfr. ult. hasi subplanulatus. Fig. Chemn. Cab. Hel. t. 119. f. 5-6.

(IIelix 4, Jacosta, spec. 223--225.)

**) Testa basi valde convexa, peristoma marginibus contimeis.

275. H. newka Dorns \& Heys. (1862): t. perforata, pyramidato-turrita; anfractus bicarinati; diam. $9^{1 / 2}$, alt. $11 \mathrm{~mm}$. (Ins. MIallorca.) T. confertissime costulata, griseo-flavida, castaneo-maculata et zonata, spira obtusa, anfr. $6^{1} / 2-7$, bicarinati, carinis obtusis, sulco divisis, apertura perobliqua, lunato-ovalis, peristoma superne rectum, basi breviter reflexum, marginibus callo junctis. (D. \& II.) 
276. H. majoricensis DoHRN \& Hern. (1862): t. mediocriter umbilicata, pyramidata; anfractus unicarinati; diam. 10, alt. $7 \mathrm{~mm}$. (Ins. Mallorca.) T. obtusa, costulata, griseo-flavida, castaneo-maculata et fasciata, anfr. $5^{1 / 2}$, modice convexi, ult. antice descendens, apert. perobliqua, lunato-ovalis, peristoma superne rectum, basi incrassatum, breviter reflexum, marginibus callo tenui junctis. (D. \& H.)

7)'Cochlicella (Fér.) Risso: testa perforata vel rimata, turrita, bulimoidea, non carinata, costulato-striata; apertura rotundatoocalis, peristomate simplici. (Butimus gen. Pfr.)

277. H. conoidea DRP. (1801): t. globoso-conica, striatula; anfr. 6, convexiusculi; apertura ovato-rotundata, lunata, marginibus subæqualibus; diam. 5-7, alt. $6-9 \mathrm{~mm}$. (Regio mediterrana.) T. aperte perforata, opaca, alba, unicolor vel fasciis fuscis multimode variegata, anfr. superiores carinati, ultimus major, $2 / 5$ longitudinis sulæquans, rotundatus et quasi turgidulus, inferne obtuse angulatus. Syn. H. fibula Wood, Bul. solitarius Pfr. (nec Poir. quæ forma subscalaria H. candidulæ, teste cel. M. Tand.). Fig. Rossm. Icon. f. 376.

278. H. pringi PFr. (1854): t. conica, irregulariter striata et punctata; anfr. 7, convexi; apertura fere circularis, margine dextro perarcuato, columellari substricto; diam. 5, alt. $8 \mathrm{~mm}$. (Lusitania, prope Peniche.) T. anguste umbilicata, solida, cretacea, fascia fusca basali plerumque ornata, vel corneo marmorata, anfr. ult. rotundatus, obtuse angulatus, $1 / 3$ longitudinis non attingens. (Pfr.)

279. H. ventrosa FÉr. (1821): to ovato-conica, plicatulostriata; anfr. 7, planiusculi; apertura ovato-lunata, marginibus inæqualibus; diam, 6, alt. $9 \mathrm{~mm}$. (Gallia merid., Lusitania.) T. subperforata, albida vel grisea, strigis et fasciis fuscis vel corneis varie ornata, anfr. $7-8$, ultimus major et subrotundatus, 1/3 longitudinis vix superans, apertura oblique semiovalis, marginibus inæqualibus, columellari strictiusculo. Syn. Bulimus ventricosus Drp. 1801, $H$. acuta Webb 1833, H. bulimoides M. Tand. 1855. ? H. carinula Gmel. 1789, H. cretaceu Chemn. 1778. Fig. Rossm. Icon. f. 377. - Variat 1) major: d. 8, a. $12 \mathrm{~mm} .:$ 2) minor (H. agnata Z.); 3) moutoni: t. transparens, rufescenti-cornea, unicolor.

280. H. acuta MüLL. (1774): t. conico-turrita, irregulariter striata; anfr. 9-11, convexiusculi; apertura subovalis, margine dextro arcuato, columellari strictiusculo; diam. $5 \frac{1}{2}$, alt. $15 \mathrm{~mm}$. (Regio mediterranea et Britannia.) T. rimato-perforata, albida vel grisea, fasciis et strigis fuscis varie ornata, infra suturam sat profundam fortius striata, apertura margine columellari membranaceo, su- 
perne reflexo, perforationem fere claudente. Syn. H. barbara Lin., Turbo fasciatus Penn. 1777, H. bifasciata Pult. 1799, Bul. variabilis Hartm. 1815, Cochl. meridionalis Risso 1826, Bul. ventricosus 'Turt. 1831. Fig. Rossm. Icon. f. 378. - Variat 1) albida, umicolor, vel fascia basali obscura (Bul. litoralis Brum.); 2) major, articulatim nigro-fasciata; d. 19 , a. $6 \mathrm{~mm}$. (Bul. articulatus Lam. 1822, B. acut. var. maritimus Desmoul. 1827); 3) minor, ventricosior (Bul. acutulus Z.)

15. Tachea LEACI. Testa magna, imperforata, subglobosa, scpius fasciis 1-5 distinctis ornuta; anfr. 5-6 convexiusculi, ultimus convexus, tumidus, antice deftexus; apertura obliqua, late lunaris, labiata, peristomate sapius patulo et margine columellari stricto.

IIabitat in silvis, hortis et fruticetis, in foliis et truncis arhorum. - Fossilis in stratibus miocenicis et pleistocenicis.

† Testa non spiraliter lineata; apertura margine exteriore forte curvato, reflexo-patulo.

281. H. vindobonensis C. PFr. (1828): t. transversim costulato-striata; apertura late rotundato-lunita, margine basali strictiusculo perlate reflexo-adnato, albolabiata, limbo hepatico, diam 23-24, alt. $17-18 \mathrm{~mm}$. (Eur. orient., præcipue Austria tota et 'Turcia.) T. perraro perforata, lutea vel luteo-albida, כ̌-fasciata, fascia infima latissima et umbilicum arete obsidente, costulis modo validis sat regularibus, modo paullo apparentibus, apertura vix angulata, peristoma late expansum, margine basali intus ensiforme compresso, medio albo subdenticulifero. Syn. $H$. austriaca Mhlf. ap. Rossm. 1835, $\boldsymbol{H}$. arvensis Kryn. 1837, H. mutabitis Hartm. 18t0. Fig. Rossm. Icon. f. $7 \& 495$. Variat 1) minor, globosa, d. 15-16, a. $15 \mathrm{~mm}$. (H. silv. v. alpicola Fér., Rossm. f. 495 e); 2) conoidea: d. 25, a 24 mm.; 3) pallescens Fér.: fasciis obsoletis, raro; 4) nigra (anfr. 5 confluent.), sutura albocingulata, regio umbilicalis lutea (perraro, Dalm. \& Albania.) (Foss.)

282. H. nemoralis MÜLL. (177t): t. transversim striatula; apertura angulato-lunata, pariete fusco, margine basali stricto calloso, carneo-Iabiata, limbo ca'staneo-nigro; diam. 20-23, alt. 16 $-17 \mathrm{~mm}$. (Eur. occicl. et media.) T. subglobosa, nunc tenuissime striata, nune læviuscula, sæpius plus minus scrobiculata vel obsolete malleata, rarissime lineolis obsoletis paucis spiraliter notata, nitida, colore maxime varia, fasciis $1-5$ (rariss. $6-7$ ) vel concolor, peristoma late expansum, margine basali longo, nigro. (Animal sagitta longa recta aciebus acutis munitum). Syn. $H$. mutabilis Hartm. Fig. Rossm. Icon. f. 5,298, 404. Variat 1) albolubiata, limbo pallide hepatico; t. sæpissime stramineo-lutescens, concolor (Dan., Germ., Carinth. \&c.); 2) gal= lice: t. maxima plerumque fasciata, apertura labio, limbo et margine 
basali sæpissime candidis, raro labio albo, limbo et margine purpurascentibus; d. 32-33, a. 20-23 mm. (Gall. merid.); 3) libellula Risso (1826), t. diaphana, citrina, perist. rubro-violaceo; 4) conoidea Cl. (1871), t. spira conico-elata, d. 22, a. $18 \mathrm{~mm}$. (Bavar.); 5) creticola Mörch (1861), t. crassissima, ponderosa, albida, subtus flavida, plerumque late fusco-fasciata, umbilico sæpe aperto, diam. ad $30 \mathrm{~mm}$. (Succ, Dan.); forma minor, cretacea, fasciis hepaticis sæpe latissimis ornata in Irlandia, White Strand. (Foss.)

- var. marginata WesterL. (1871): t. albo-labiata, brunneo-limbata, margine basali acuto, stricto, tenui, a margine exteriore incisura profunda disjuncto. (In plerisque terris, sed raro.)

- var. porrecta WestenL. (1871): t. sutura antice non subito descendente et anfractu ultimo non prorsus deflexo, apertura adeoque magis rotundata, margine exteriore supra fascias duas infimas affixo. (Suecia Malmö.)

- var. Zucifuga Z. ap. Hartм. (1840): t. gigantea, fasciata vel concolor, scrobiculata, malleata, transversim striata et lineolis sub lente minutissimis et creberrimis spiralibus signata; apertura labio pallide carneo et limbo hepatico, vel labio purpureo et limbo nigro; d. 30, a. $22 \mathrm{~mm}$. (Italia.) syn. H. nemor. v. apennina Stab., H. genuensis Porro.

283. H. hortensis MüLL. (1774); t. minor, transversim striatula vel lavigata; apertura angulato-lunaris, pariete pallido, marnine basali stricto calloso, labio et limbo albis; diam. 19, alt. 13-15 mm. (Eur. bor., occ. et media ad Alpes et Pyrencas.) T. globosa, lineolis paucis obsoletis spiraliter rarissime notata, colore varia, unicolor vel $\mathbf{1 - 5}$ (raro 6) fasciata, peristoma late expansum, margine. (Animal sagitta cuspide leviter incurvo, basi capitata, aciebus dilatatis canalibus profundis sejunctis). Syn. H. nemoralis Lin. 1758! Fig. Rossm. Icon. 6, 299. Variat 1) minor: d. 14, a. $9 \mathrm{~mm}$.; 2) Iudoviciana d'Aumont: t. minor, membranacea, pellucida (Islandia, Dania, Bavar., Gall.); 3) crassa: t. ponderosa, crassa, spira elevata, conica, anfr. ult. antice parum deflexo, apertura minore (Suecia, Skåne); 4) trochoidea Cl.: d. 19, a. $17 \mathrm{~mm}$.; 5) arenicola Macg. (1844): t. tenuis, pellucida, pallide lutescens, fasciis albis opacis (Scotia); 6) fusco-labiata: t. pallide labiata, fusco-limbata, pariete aperturali subfuseo $(H$. hybrida Poir., H. sauveuri Colb.). (Foss.)

- perforata WesterL. (1871): t. lutea, 5-fasciata, valde perforata. (Succ. ins. Gotland.)

it Testa distincte subregulariter lineata, upertura margine exteriore extense arcuato, recto. 
284. $H$. sylvatica DRP. (1801): t. transversim vix striatula, albida, interrupte fasciata; apertura rotundato-lunata, albo-labiata, albo- vel hepatico-limbata, pariete pallido, loco umbilicali roseo, margine basali antice curvato; diam. 19, alt.'15 mm. (Gallia merid., Helvetia, Itatia bor., German. merid.-occid,) T. imperforata, depresse-globosa, subnitida, apertura margine basali intus compresso, rectiusculo, antice non angulum cum exteriore formante. Syn. H. vindobonensis Dup. 1818. Fig. Rossm. Icon. f. 496. - Variat 1) major: d. 24-25, alt. 18-20 mm.; 2) alpicola M. T.: t. minor, globosior, magis striata et colorata; 3) montana Stud. (1789): t. minor, magis depressa, valde striata. (Foss.)

16. Macularia ALB. Testa magna, cxumbilicata, (raro obtecte perforata, rarissime umbilicata), depresse-globosa, laviuscula, fasciis sape interruptis ornata; anfr. 5, ultimus antice sapius valde descendens; apertura obliqua, diagonalis, oblonga, peristomate reflexo, labiato, margine columellari dilatato, calloso.

Habitat in campis aridissimis sterilissimisque Europæ maxime meridionalis, in scopulis, ad radices fruticum vel sub lapidibis. - Fossilis in stratibus miocenicis et strato pliocenico medio.

+ Testa lineis spiralibus ornata.

*) Apertura intus et paries aperturatis pallida.

a) T'esta globoso-depressu, quinque-fusciata vel concolor.

285. H. codringtoni Gray (183t): t. maxima vel magna, imperforata vel semiobtecte perforata, globosa; anfr. 6, primi regulariter accrescentes, ultimus ampliatus, depresso-convexus; apertura semiovali-lunata, peristomate albo, incrassato, reflexo, margine basali late dilatato, prope columellam incrassato; diam. 50, alt. 30-40 mm. (Grecia.) T. solida, striata, supra sub Jente obsolete spiraliter lineata, fusco-cornea et albida, irregulariter strigata ct marmorata, vel fasciis 2 vel 4 nigrescentibus interruptis, ornata, spira depresso-conoidea, anfr. convexiusculi, ultimus antice regulariter descendens, infra prope columellam convexus, apertura perobliqua, margine columellari valido, breviter arcuato, intus cornea, nitida. Syn. H. ferussaci Jan, H. spiriplana Desh., H. navariensis Gray. Fig. Rossm. Icon. f. 369 a, 6.; Rev. Zool. 1857. t. 9. f. 1-3. (Bg.)

-- var. eucineta Bourg. (1857): t. depressa, corneo-albida, 3 zonis corneo-nigrescentibus, inaqualibus, anfr. ult. antice subito descendens, infra depressulus, peristoma undique valde re- 
flexum, margine columellari valido, dentifero, arcuato; d. 45-50, a. $25 \mathrm{~mm}$. (Bg.) Fig. Rev. Zool. 1857. t. 9. f. 4-6.

- var. eupoccilia Bourg. (1857): t. fragilis, sat diaphana, globosa vel depressa, corneo-albida, irregulariter fasciata, anfr. ult. antice maxime descendens, infra depressulus, apertura dilatato-semiovata, peristomate leviter incrassato, acuto, margine columellari valido, recto, paullulum reflexo; d. 48-52, a. 28-35 mm. (Bg.) Fig. Rev. Zool. 1857. t. 10, f. 1-3.

- var. euchromia Bourg. (1857): t. parum solida, compressa, corneo-albida, zonis duabus æqualibus ornata, anfr. ult. antice maxime descendens, infra valde umbilicali-depressulus, apertura semiovata, peristomate non reflexo, paullulum incrassato, margine columellari valido, fere recto, paullulum reflexo; d. 47 , a. $22 \mathrm{~mm}$. (Bg). Fig. Rev. Zool. 1857. t. 10. f. 4-6.

- var. parnassia Rotr (1855): t. imperforata vel semiobtecte perforata, globoso-depressa, cæsio-albida, medio 1-5fasciata, superne distinctius spiraliter lineata, anfr. ultimus valde descendens, infra antice tumidus, apertura angulato-late lunaris, peristomá margine exteriore vix patulo, basali horizontali, fere recto; d. 30-35, a. $17 \mathrm{~mm}$. Fig. Rossm. Icon. f. 897. Variat magis globosa: d. 35 , a. $25 \mathrm{~mm}$.

286. H. guiraoana Rossu. ap. PFr. (1853): t. aperte (vel semiobtecte) umbilicata, depressa; anfr. 4-41/2, celeriter accrescentes, ultimus subdepressus, non dilatatus; apertura obovatolunata, roseo- vel albo sublabiata, margne basali breviter reflexo; diam. 23-28, alt. 10-12 mm. (Hisp.) T. arcuatim striatula, supra tenuiter decussata, albida, fusco-quinquefasciata, fasciis superioribus 3 maculose solutis vel evanescentibus, anfr. convexiusculi, apert. perobliqua, margine supero recto, acuto, basali obsolete dentifero, angusto. Fig. Rossm. Icon. f. 799.

- var. angustata Rossm. (1854): t. minor, umbilico angustiore, marginis columellaris lamella lata semiobtecto. Fig. Rossm. Icon. f. 798 .

287. H. omphalophora DUT. (1867): t. profunde umbilicata, supra compressa; anfr. 4-5, celerrime accrescentes, ultimus maximus, rotundato-compressus; apertura oblonga, infra rotundata, vix Junata, peristomate recto, albo, paullulum incrassato; diam. $30-32$, alt. $14-15 \mathrm{~mm}$. (Corsica.) T. supra vix eonvexiuscula, sat tenera ac nitida, eleganter striatula, rufo-olivacea ac tribus zonulis castaneis 
ornata, apertura perobliqua, margine columellari dilatato, reflexo, numquam umbilicum obtegente.

288. H. alonensis F́́r. (1821): t. obtecte perforata (vel exumbilicata), depresse globosa; anfr. $4^{1} / 2$, celeriter accrescentes, ultimus maximus, tumidus, dilatatus; apertura ampla, rotundatolunata, albo-sublabiata, margine supero et basali arcuatulis, vix expansis, hoc angusto, columellari late reflexo; diam. 27-33, alt. 16-19 mm. (Hisp.) T. albida vel luteola, irregulariter striata, utrinque dense et forte decussata, varie fusco-fasciata vel marmorata, vel concolor, anfr. convexiusculi. Fig. Rossm. Icon. f. 497, 498, 781 -788 .

- var. lorcana Rossu. (1854): t. umbilicata, margine exteriore expanso, reflexó. Fig. Rossm. Icon. f. 789.

- var. campesina EzQ. ap. BroNe (1845); t. multo major, aperte umbilicata, striis transversis et lineis spiralibus confertim reticulata, peristoma late expansum, undique liberum, marginibus conniventibus, callo subconnexis, columellari umbilicum semioccultante; d. 40, a. $36 . \mathrm{mm}$. Fig. Rossm. Icon. f. 790. Forte species.

289. H. loxana Rossm. (1854): t. exumbilicata (rariss. subumbilicata), subglobosa; anfr. $4 \frac{1}{2}$, celeriter accrescentes, ultimus basi planiusculus; apertura lunato-rotundata, albo-sublabiata, marginibus conniventibus, basali strictiusculo, calloso; diam. 28, alt. $14 \mathrm{~mm}$. (Hisp.) T. albida, cinereo-nebulosa, rufo- 5 fasciata, fasciis superioribus 3 interruptis, subtilissime decussata. Fig. Rossm. Icon. f. 793. - Variat paullo minor, globosior, , fasciis omnibus maculose interruptis. Fig. Rossm. Icon. f. 794.

290. H. nebrodensis Pirajno (1842): t. obtecte perforata, depressa; anfr. $5-5 \frac{1}{2}$, regulariter accrescentes, ultimus non dilatatus, antice constrictus; apertura ovalis, parva, intus fuscidula, albosublabiatum, margine basali leviter curvato, reflexo-appresso; diam. 20-25, alt. $10-13 \mathrm{~mm}$. (Sicilia, "in Nebrodum cacuminibus".) 'T'. nitida, unicolor alloida vel interrupte fusco-fasciata, minutissime decussata, margo basalis interdum subdentata. - Clar. Kobelt opinat $H$. intusplicatem Pfr. 1851, "in monte Chelmos Arcadize" (t. umbilicata, depressa, striata et lineis spiralibus confertissimis sculptata, coerulescenti-alba, apert. subtetragono-ovalis, intus carnea, margine supero recto, basali intus plica elongata oransversa stricta munito, extus reflexo, columellari fornicato-reflexo; d. 31 , a. $14 \mathrm{~mm}$.), formam H. nebrodensis esse.

291. H. alcarazana Gurao ap. Rossir. (1854): t. exumbiicata, depressa, subglobosa; anfr. $4^{1} / 2$, celeriter accrescentes, ul- 
timus basi convexiusculus; apertura rotundato-lunaris, roseo-labiata, margine basali adpresse calloso, leviter arcuato; diam. 20-22, alt. 11-12 mm. (Hisp.) T. subtilissime decussata, albido-lutescens, fúsco- 5 fasciata, fasciis 3 superis maculosis. Fig. Rossm. Icon. f. 795 .

292. H. niciensis Fér. (1821): t. obtecte perforata, globoso-depressa; anfr. 5, sat forte accrescentes, ultimus basi convexus; apertura late lunato-ovalis, intus et labio lilacinis, margine basali intus declivo, compresso, acuto, leviter curvato; diam. 23-24, alt. 11-14 mm. (Gallia merid., Nizza; Toulon, Grasse, Entrevaux: M. T.) T. subcoerulescenti-alba, nitida, maculose brunneo5-fasciata, fascia tertia maxima, obscura, cæteris pallidis, obsoletis supra tenue striata et spiraliter lineata, apertura margine basali appresse dilatato. Syn. H. nicaensis Rossm. 1836. Fig. Rossm. Icon. f. 244, Moq.-Tand. Hist. t. 12. f. 4-7. Variat 1) unicolor: Rossm. f. 601, 2) minor: Rossm. f. 602. (Spec. descripta e Nizza habeo.)

- var. dupuyi mh: t. depressa vel subgloboso-depressa, perforata (foramine quandoque per callum obtecto), striata, striis supra exaratis, sine vestigio linearum spiralium, albescens -vo albido-lutescens, superne spiraliter corneo-maculata, apertura ovatolunata, intus et labio lilacinis, corneis vel carneo-lutescentibus; d. $19-24$, a. $9-11 \mathrm{~mm}$. ("d. 20 -26, a. $10-18 \mathrm{~mm}$." Dup.) - Gall. merid. ad Grasse: spec. plura clariss. Dupuy misit.' Syn. H. niciensis Dup. 1848. Fig. Dup. Hist. t. 5. f. 3.

293. H. carthaginensis Rossm. (1854): t. exumbilicata, subgloboso-depressa; anfr. $4^{1} \frac{1}{2}$, rapide accrescentes, ultimus antice valde dilatatus; apertura obovato-lunata, albolabiata, margine basali calloso, strictiusculo, angusto; diam. 21-24, alt. $10-11^{1 /} / 2$ mm. (Hisp.) T. striata, minute decussata, lutescens, corneo-variegata, fasciis 5 ornata, superioribus 3 plerumque maculose solutis, spira brevissime conoidea, anfr. ult. basi planiusculus, peristoma expansum, marginibus subparallelis, columellari dilatato, reflexo, adnato. Fig. Rossm. Icon. f. 791, 792.

294. H. marmorata FÉR. (1821): t. exumbililicata, globosodepressa; anfr. 5, celeriter accrescentes; apertura late lunaris, peristomate simplici, albo vel roseo, margine dextro patulo, columellari appresso, subdilatato, albo; diam. 20, alt. $11 \mathrm{~mm}$. (Gibraltar.) T. lutescenti-cornea, interrupte 5 -fasciata, fasciis 3 superis dissolutis fusculo albidoque marmorata. Fig. Rossm. Icon. f. 243.

(H. platychela Mke var.)

ß) Testa globoso-turbinata, quadrifasciata vel concolor. 
295. H. platychela Mrke (1830): t. subglobosa, unicolor vel maculose 4 fasciata: apertura truncato-rotundata, peristomate late expanso-reflexo; diam. 24, alt. $17 \mathrm{~mm}$. (Sicilia.) T. exumbilicata, cæesio-albida, transversim vix striatula, superne (ad fasciam infimam) dense longitudinaliter lineata, spira conoidea, anfr. $5^{1 / 2}$, convexi, ultimus convexus, paullo tumidus, apertura horizontaliter extensa, intus luteseens, peristoma candidum, nitidum, margine basali late reflex oadnato, strictiusculo, compresso, intus declivo, subdentato. Syn. $H$. jratexta Jan. 1832. Fig. Rossm. Icon. f. 443-445. Variat spira conica, d. 20 , a. $18 \mathrm{~mm}$.

- rosalia Ben. ap. Alb. (1860): t. basi admodum convexa, maculose et irregulariter fulvo- 3-fasciata, sutura profunda, peristoma breviter expanso-reflexum. Fig. Ben. Ill. Sic. t. 1. f. 12.

- var. iparia Ben.: t. subdepressa, anfractibus supernis planulatis, ultimo mediocriter convexo, subcarinata. Fig. Ben. Ill. Sic. t. 1. f. 13 .

- var. spuria (Parr. ex.): t. globoso-depressa, lutescentialbida, 4-fasciata, superne densissime transversim et spiraliter striata, adeoque creberrime subalutacea; d. 22, a. $12 \mathrm{~mm}$.

296. H. sicana FẺr. (1822): t. conoideo-subglobosa, unicolor vel 4 -fasciata, cervice constricta; apertura parva, lunato-rotundata, peristomate angusto reflexo; diam. 21, alt. $18 \mathrm{~mm}$. (Sicilia.) T. exumbilicata, alba, rarius fusco-fasciata, substriatula, superne dense longitudinaliter lineata, spira alta, conoidea, anfr. 6, planiusculi, ultimus rotủndus, inflatus, apertura subverticalis, irregulariter (angulatim) rotundato-lunaris, intus alba, peristoma candidum, margine exteriore in pariete longe producto, basali brevi, gibbo, sæpius subdentato. Syn. H. soluta Z. ap. Phil. 1836. Fig. Rossm. Icon. f. 446, 447 (typ.), 594, 595 (varr.) Variat 1) forma: d. 18, a. $20 \mathrm{~mm}$.; d. 22, a. $20 \mathrm{~mm}$.; 2) minor: t. tota lactea vel unifasciata, d. 14, a. $13 \mathrm{~mm}$.

**) Apertura intus et paries aperturalis nigra.

297. H. lactea MIÜLL. (1774): t. depresse-globosa, transverse dilatata; apertura truncato-ovalis, peristomate expanso, nigro-castaneo, margine basali substricto, calloso-subdentato; diam. 29-39, alt. 17-23 mm. (Hisp., Lusitania, ins. Balear.) T. exumbilicata, creberrime et tenuissime spiraliter lineata, Intescenti-grisea, varie fusco-fasciata et albo-atomata, rarius unicolor, anfr. $4^{1 / 2}-5$, convexiusculi, celeriter accrescentes, ultimus latior; rotundatus, apertura rotundato- late lunata, intus nigra, nitida, peristomate obtuso, marginibus plerumque subparallelis. Sagitta animalis subrecta, apice paullisper curvata, c. $4 \mathrm{~mm}$. longa. Syn. $H$. irrorata Say, H. rugulosa Risso. Fig. Rossm. Icon. f. 802, 803. Variat præ- 
cipue in Hispania 1) unicolor, alba; 2) maura Guirao, t. fasciis confluentibus nigrobrunnea, unicolor (Rossm. f. 80t.); 3) turturina Guirao, unicolor, isabellina, peristomate læte fusco (Rossm. f. 805); 4) sevillana Gratel., t. minor, minus depressa, isabellina fere unicolor vel zonis obsoletiusculis, atomatis, anfractibus lente crescentibus, apertura parvula; d. sæpe vix $20 \mathrm{~mm}$. (Rossm. f. 807); 5) atomaria Schum., t. fasciis obsoletis fere omnino confluentibus, punctis copiosissimis albis obtecta, peristomate fere rectangulare patente (Rossm. f. 801);6) murcica Rossm., t. maxima, apertura valde dilatata et peristomate valde reflexo (Rossm. f. 800); 7) minima: d. 20, a. $13 \mathrm{~mm}$. (Rossm. f. 808).

298. H. punctata MüLL. (1774): t. subdepressa; apertura truncato-ovalis, subangulata, dextrorsum dilatata, peristomate albo, reflexo, margine basali stricto, rarius obsolete dentato; diam. 28 -39, alt. 16-20 mm. (Hisp. merid.; in Pyrenæis orientalibus introducta.) T. exumbilicata, tenuissime spiraliter lineata, albida, 5-fasciata et albo-atomata, vel subunicolor fusca, albopunctata, anfr. 5, convexiusculi, celeriter accrescentes, ultimus rotundatus, apertura intus nigra, nitida, peristomate obtuso, margine dextro arcuato cum basali stricto angulum formante. Sagitta animalis sursum fortius curvata, c. $5 \mathrm{~mm}$. longa. Syn. H. lactea Fér., Mich., Dup. \&c. Fig. Rossm. Icon. f. 545, 548. Variat punctatissima Jen., t. maxima, dense punctata, limbo peristomatis modo pallido.

299. H. apalolena Bourg. (1867): t. plus minusve depressoglobosa; apertura lunata, transverse oblonga, peristomate patulo, intus albidulo, margine basali stricto, non calloso; diam. 33-36, alt. 20-24 mm. (Hispania, Gallia merid., ins. Mallorca.) "Differt ab $H$. punctata forma depressa, minus globosa, testa tenui, lævigata, vix striata, apertura magis transverse oblonga, anfractu ultimo rapidius descendente, omnibus celerius crescentibus et minus convexis; ab $H$. lactea distinguitur testa fragiliore, tenuiore, læviore et nitidiore, colore constante (t. griseo-castanea, duabus vel tribus zonulis pallidioribus parum perspicuis circumornata et undique passim lacteo-punctata), apertura minus obliqua, apertiore, margine columellari recto, non tuberculoso, anfractibus rapide et perfecte regulariter accrescentibus" (Bourg.). Fig. Bourg. Moll. nouv. t. 35. f. 1-5.

(H. balearica var.)

if Testa lineis spiralibus destituta.

300. H. vermiculata MüLL. (1774): t. globoso-depressa vel globosa, tenuissime striata et impressionibus variis et varie positis scrobiculata; apertura ovato-late lunata, peristomate albo, sublabiato, margine basali stricto, subconvexo; diam. 30-35, alt. 17-21 mm. (Regio mediterranea.) T. imperforata, solida et opaca, lineolis punctisque incrassatis albis quasi subreticulata, lutescens, fa- 
seiis rufis (superis sæpissime tæniatis) ornata, anfr. 5-6, convexiusculi, ultimus rotundatus, subventrosus, peristoma late expanso-reflexum, marginibus subremotis, basali compresso, calloso. Syn. H. lactea Hartm. Fig. Rossm. Icon. f. 301, 499, 500. Variat 1) multimode fasciata et unicolor; 2) limusiana Ben. (H. linusæ Cale.), testa minor, subglobosa, solidior, apertura ante lunaris (Sicil., Malta); 3) subangulata Iss., t. minor, solidior, apertura subangulata (Malta); 4 thalessina P'orro, t. tenuis, fragilis (Sardinia). Variat rarissime umbilico aperto.

301. H. companyoni Aleron ap. Dup. (1848): t. depressa, tenuissime sub lente striatula; apertura transverse ovata, parum lunata, extus dilatata, subangulata, peristomate candido, labiato, margine basali stricto; diam. 15-20, alt. 8-12 mm. (Pyrence orient., ins. Balear.) T. imperforata, solidula licet sat tenuis, subnitida, albida, fasciis 5 valde interruptis ornata, subtus candida et fusco-radiata, anfr. $4 \frac{1}{2}-5^{1} / 2$, convexiusculi, apertura intus albida, margine exteriore subpatulo, basali calloso. Syn. H. companyoni Aleron 1837 (nomen), H. hispanica var. pyrenaica Rossm. 1839. Fig. Rossm. Icon. f. 591.

302. H. balearica Z. ap. Prr. (1853): t. globoso-depressa, tenue arcuatim striata; apertura transverse ovato-lunata vel late rotundato-lunata, peristomate hepatico margine basali substricto; diam. 26-30, alt. 12-15 mm. (Hispania, ins. Balear.) T. imperforata, fusculo-albida vel fulvida, castaneo-maculata, variegata ct 5-fasciata, fasciis supremis maculose abruptis vel laceratis, anfr. 5, convexiusculi, sutura impressa, apertura perobliqu, intus transversim late margaritacea, margine exteriore subpatulo. Syn.. H. hispanica Partsch ap. Rossm. 1838. Fig. Rossm. Icon. f. 460.

- var. violacea Rossir. (1854): t. depressior, violaceo-labiata, margine exteriore aperturæ valde deflexo. Fig. Rossm. Icon. f. 796 .

- var. pulchella Rosss. (1854): t. multo minor, globosior, distincte spiraliter lineata, supra luteo-brunnéa, eleganter albo picta et obtuse fasciata, infra albida, bifasciata, labium violaceum. Fig. Rossm. Icon. f. 797.

303. H. asteia Bourg. (1863): t. depresso-ventricosa; globosa, leviter striatula et vix passim obscure malleata; apertura lunato-oblonga, intus brunnea, peristomate pallide brunneo; diam. 29, alt. $17 \mathrm{~mm}$. (Hisp. ad Madrid.) T. imperforata, cretacea, violaceo-candida ac passim rarissime brunneo-maculata, anfr. $5_{12}^{11}$, convexiusculi, celeriter accrescentes, ultimus maximus, dilatatus, rotundatus, valde perdeflexo-descendens, peristoma late expansum, incrassatum. Fig. Rev. Zool. 1863. t. 7. f. 5-8. (Br.) 
304. H. graëllsiana PFr. (1848): t. depresse-globosa, lævigata; apertura ampla, lunato-ovalis vel rotundata, peristomate rufo, sublabiato, margine basali arcuato; diam. 20-22, alt. 14 $\mathbf{- 1 5} \mathrm{mm}$. (Ins. ILajorca.) T. imperforata, tenuis, sericina, griseocarnea, maculose quincuefasciata, fasciis interruptis angustis corneo et albido variegatis, anfr. 4, convexiusculi, rapide accrescentes, ultimus ventricoso-inflatus, antice breviter deflexus, apertura perobliqua, peristoma breviter expansum, marginibus conniventibus; basali dilatato, plano. Syn. H. grateloupi Graells 1846 (non Pfr.) Fig. Rossm. Icon. f. 815 .

\section{(H. niciensis var. dupuyi.)}

17. Iberus Montr. Testa obtecte, raro aperte, umbilicata, orbiculata, depressa, plus minusve rugosa, albida, obsolete fasciata; anfr. 4-5, ultimus antice descendens; apertura perobliqua, oblonga; peristoma acutum, labiatum, expansum, margine columellari reflexo, sapissime superne macula rufa signato.

Habitat locis aridis, in saxis scopulisque et muris per regiones mediterraneas (Africa bor. excepta).

† Testa ecarinata (anfr. ult. rotundatus vel angulatus).

*) Testa imperforata.

a) Testa superne densissime clathrata, alutacea vel creberrime granulata.

a) T. lactea, unicolor, peristoma superne rectum.

305. H. eugenia $\mathrm{P}_{\mathrm{FR}}$. (1853): t. conoideo-depressa, minute et confertim striata; anfr. vix planiusculi, penultimus angulatus, ultimus depressus, basi convexior, antice subito deflexus; apertura rotundato-lunata, coarctata; diam. 14, alt. $8-9 \mathrm{~mm}$. (Sicilia.) T. lactea, cretacea, spira paullo conoidea, anfr. ult. antice rotundatus, apertura intus fusco-carnea, albo-sublabiata, marginibus subapproximatis, columellari dilatato, perforationem omnino vel partim claudente. Syn. $H$. calypso Ben. 1859. Fig. Ben. Ill. Sic. t. 2. f. 18.

- Tuetiana BEN. (1859): t. multo major, conferte striata, spira minus exserta, margine basali subdentato; diam. 18-20, a. 11-12 mm. Syn. H. hueti Pfr. 1859. Fig. Ben. Mll. Sic. t. 5. f. 1 .

b) T. albida, supra strigis vel fasciis interruptis fusco-marmorata, infra albida, raro tota unicolor; peristoma expansum vel reflexum. 
306. H. globularis Z. ap. Rossur. (1838): t. globoso-depressa, glabriuscula; anfractus ultimus rotundatus, infra tumidus; apertura ovali-rotundata, lunata, margine basali substricto, albo, subdentato; diam. 19-21, alt. 12-13 mm. (Sicilia et ins. IIaritino, Italia merid. ad Tarento.) T. albida, subseriatim fusco-macuosa vel unicolor, anfr. 5, convexiusculi, regulariter accrescentes, apertura irregulariter subrotunda, marginibus distantibus, exteriore reflexiusculo, albo. Fig. Rossm. Icon. f. 442. Variat 1) conspicua Ben., t. major, magis globosa, fasciis quattuor maculose interruptis signata; d. 20, a. $14 \mathrm{~mm}$. (Trapani); 2) friwaldskyi Calc. 1846, t. minor, spira minus elevata, maculis latis confertis exornata d. 16, a. $10 \mathrm{~mm}$. (Ins. Pantellaria; H. glob. v. cossurensis Ben.); 3) achatina Ben., t. unicolor; 4) latilabris m., t. minor, turbinato-globosa, quadri-fasciata, apertura angusta, angulata, peristomate undique latissime reflexo, albido nitido; d. 19 , a. $12 \mathrm{~mm}$.

307. H. signata F́́r. (1821): t. subdepresso-globosa, substriata; anfractus ultimus rotundatus, infra ad umbilicum tumidus; apertura lunato-rotundata, margine basali arcuato, calloso, non dentato; diam. 22, alt. $13 \mathrm{~mm}$. (Italia inferior.) T. alba, fasciis 3-4 maculose interruptis castaneis ornata, anfr. 5, planiusculi, peristoma patulum, sæpe fusculum, albolabiatum, marginibus subconniventibus. Fig. Rossm. Icon. f. 686 . Variat minor: d. 17-18, a. $11 \mathrm{~mm}$.

308. H. muralis MüLt. (1774): t. subdepressa, valide rugoso-striata; anfr. regulariter accrescentes, ultimus supra depressus, obtuse angulatus, infra, præsertim medio, convexior; diam. 19, alt. 9. mm. (Italia, Sicilia, Gallia merid.) T. suborbiculata, albida, fusco-maculata (costis sæpius albis, interstitiis brunneis), basi obsoletius costata, unicolor, alba, anfr. 5, convexiusculi, ultimus antice minime dilatatus, apertura transverse ovalis, intus fusca, peristoma albolabiatum, acutum, marginibus distantibus, reflexis, basali levissime arcuato, appresso. Syn. H. vugulosa Risso, H. rugosa Z., H. muratis var. Rossm., H. mur. var. costulata Ben. Fig. Rossm. Icon. f. 231 a. Variat 1) crassicosta Huet, t. supra rufescentibrunnea, valide albocostata (Sicil.); 2) crispata Ben., t. elatior, superne profunde plicata, anfr. ult. obsolete carinato (Sicil.); 3) conoidea Gentil., t. spira conoidea, d. 15, a. $12 \mathrm{~mm}$. (Ital., Firenze); 4) minor, elatior, minus rugosa. subtiliter striata, superne albo, fusco et plumbeo variegata, subtus unifasciata; d. $17^{1}{ }_{2}$, a. $10 \mathrm{~mm}$. (H. muralis a Rossm. f. 230, Ben., nec Müll.); 5) minima: d. 15, a. $8 \mathrm{~mm}$.; 6) carinata, t. anfractu ultimo utrinque compresso, medio subacute angustato.

- var. tibcriana BEN. (1859): t. unicolor lactea vel rufomaculata, supra costulato-rugosa, anfr. 5 subplanulati, obsolete carinati, spira exserta, pyramidata, apertura ovato-lunata; d. 21, a. $13 \mathrm{~mm}$. (Sicil.) Fig. Ben. Ill. Sic. t. 2. f. 17. 
- var. circumornata FÉR. (1822): t. globoso-depressa, striatula, sublævigata, rugosa, densissime alutacea, alba, fascia unica rufa infra medium et superne maculis trausversis obscuris et 3 seriebus macularum aurantio-fuscarum ornata, anfr. 5, convexiusculi, ultimus peripheria rotundatus, apertura lunato-elliptica, intus fusca, nigricans, peristoma album, subreflexum, d. 19-20, a. $11 \mathrm{~mm}$. (Ital. ad Napoli.) Variat minor, striata: d. 15, a. $8 \mathrm{~mm}$.

309. H. paciniana PhrL. (1836): t. depressa, rugoso-costulata, rugis albis, interstitiis lividis vel brunneis; anfr. sat forte accrescentes, ultimus medio acute angulatus, subcarinatus; apertura elliptico-lunaris, extus angulata; diam. 16-17, alt. $8 \mathrm{~mm}$. (Sicilia occid.) T. convexiuscula, anfr. 5, planiusculi, ultimus antice deflexus, apertura intus brunnea, peristoma album, incrassatum, margine exteriore vix patulo, basali duplo longiore breviter reflexo, arcuato, columellari dilatato, appresso. Syn, H. vieta Rossm. 1836. Fig. Rossm. Icon. f. 232.

310. H. substrigata Bourg. ap. MaB. (1868): t. depressa, costis irregularibus ornata; anfr. irregulariter crescentes, ultimus maximus, compressus, obsolete carinatus; apertura lunata, oblique ovata; diam. 18. alt. 9 mm. (Sicil. prope Marsala.) T. costis, strigis ac tuberculis minimis subquadratis, compressis, paullulum acutis ornata, cinerea, nigro-purpurascente maculata, anfr. $5-51 / 2$, depressoconvexi, primi sat lente regulariterque, cæteri celerrime crescentes, sutura impressa, ultimus antice valde deflexus, in loco umbilicali paullo inflatus, apert. intus intense rubro-pruinosa, peristoma breviter reflexum, album, sublabiatum, margine columellari late dilatato, umbilicum tegente, ad basin violaceo-cinerascente tincto. (Bg.)

311. H. abromia Bourg. ap. MAB. (1868): t. depressa, costulis confertis linearibus ornata; anfr. 4-5, subregulariter accrescentes, ultimus magnus, depresso-rotundatus; apertura late ovatorotundata; diam. 11, alt. $7 \mathrm{~mm}$. (Lombardia.) T. supra convexoplanata, infra depresso-convexa, opaca, albida, maculis rufis fulguratis marmorata, anfr. ult. antice non dilatatus, paullulum deflexus, apertara intus pallide rufo-tincta, peristomate ${ }^{-}$albo expansiusculo, subincrassato, margine columellari paullo reflexo.

312. H. orgonensis Philb. ap. Moq.-TAND. (1855): $t$. depressa, dense pertenue striata, hinc inde costulata, minime rugosa; anfr. 5, convexi, irregulariter crescentes, ultimus maximus, rotundatus; apertura lunata, ovato-rotundata; diam. 18, alt. $9 \mathrm{~mm}$. (Gallia merid.; Sicilia?) T. supra planata vel planato-convexa, alba, strigis maculisque undulatis longitudinaliter fusco-marmorata, 
subtus unicolor, anfr. irregulariter (primi lente, ultimi celerrime) crescentes, ultimus in loco umbilicali inflatus, antice regulariter et valde descendens, apertura intus purpurascens, peristomate albo, expanso reflexoque, margine columellari adpresso. Syn. H. undulata Mich., nec Fér., H. serpentina var. panormitana Ben., teste Kob. Fig. Dup. Hist. t. 5. f. 1 (H. mur.), M. 'T. Hist. 11. f. 36 (H. mur. var. undul.).

313. H. abræa Boung. ap. Mab. (1868): t. depressa, tenuissime striata ac costulis irregularibus ornata; anfr. 4-5, convexodepressi, irregulariter celeriterque accrescentes, ultimus permagnus, compresso-rotundatus; apertura oblongo-ovata, lunata; diam. 14 -17, alt. 9-10 mm. (Lombardia.) T. supra cinereo-albida, maculis et punctis violaceis marmorata, subtus unicolor, olivacea, anfr. primi rapidi, creteri magni celerrime crescentes, ultimus demum subito deflexus, in loco umbilicali inflatus, apertura intus rufo-purpurascens, peristoma leviter expansum, intus paullo incrassatum, albolutescens. (Bg.)

314. H. grohmanni PinL. (1836): t. depressa, carinata, subtiliter striata; anfr. $4^{1} / 2$, subplanulati, ultimus antice subdeflexus; apertura securiformi-lunata; diam. 21, alt. 91/2. mm. (Sicilia.) 'T. albida, 3-4 seriatim fusco-maculata, orbiculata, carina antice evanescente, apertura intus suturate fasciata, extus angulata, peristoma simplex, margine exteriore deflexo, basali reflexo, calloso. Fig. Rossm. Icon. f. 448. - Ols. Hæc et præcedentes quattuor species forte formæ Helicis muralis.

B) Testa supcrne transversim malleata rel lineolis spiratibus obsoletis irregulariter notata, anguste fasciata.

315. H. splendida Drr. (1801): to depressa, lavigata vel obsolete striata; anfr. rapide accrescentes, ultimus depressus, dilatatus; apertura ovali-lunata; diam. 20, alt. $11-12 \mathrm{~mm}$, (Gall. merid. et Hisp.) T. solidula, licet tenuis, subpellucida, albida, fulvida vel isabellina, fasciis $1-5$ perangustis, inferioribus 2 obscuxis, superioribus pallidis, evanescentibus vel raro confluentibus, anfr. 4 $-4^{1} / 2$, convexiusculi, ad suturam vix perspicuam omnino plani, ultimus subtus paullo convexior, antice lente descendens, apertura intus alba, peristoma subrectum, vix patulescens, intus incrassatum, album, marginibus sæpius subparallelis, raro basali arcuatulo, columellari sæpissime roseo dilatato foramen angustissimum obtegente. Fig. Rossm. Icon. f. 238. Variat 1) roseo-labiata Piossm. f. 609, t. peristomate roseo; 2) spluacelate IVebb. ap. M. T'and., testa tota álba, unicolor.

r) Testa non vel vix spivaliter lineata, interrupte fasciata et maculis obscuris variegata (2el unicolov). 
316. H. serpentina Fér. (1822): t. depressa, striata; anfr. celeriter accrescentes, ultimus depresso-rotundatus, dilatatus; apertura rotundato-lunata, intus profunde margaritacea, nitida, albolabiata, regione umbilicali et pariete castaneis; diam. 17, alt. 8 -9 mm. (Italia, Gallia in Provence.) T. formæ H. splendidæ æqualis, late convexiusculus, imperforata vel raro subperforata, albida, supra maculis rufobrunneis seriatim positis et strigis dense variegata et marmorata, infra fasciis 2 abruptis sæpius ornata, anfr. 5, ult. antice descendens, sutura sat impressa, apertura extus dilatata, peristoma patulum, margine columellari angusto, obliquo, stricto. Fig. Rossm. Icon. f. 239. -- Variat submarmorata: t. nigricanti-marmorata, fasciis confluentibus, strigis fulminatis junctis; d. 20, a. 11-12 min. (Rossm. f. 222; H. marmorata sæpe in coll., nec Fér.)

- var. hospitans Bon. ap. Rossur. (1836): t. depressa, spira convexo-conoidea, striąta, alba v. lutescens, seriatim maculis ornata; anfr. 5, ult. magnus, rotundatus, infra antice tumidus, apertura lunata, oblongo-rotundata, peristomate leviter expanso; d. 21, a. $12 \mathrm{~mm}$. (Sardinia, Corsica.) Syn. H. carce Cantr. 1840. Fig Rossm. Icon. f. 240. Variat jaspidea M. T., t. superne maculis distantibus marmorata (Rossm. f. 241.)

- var. isilensis Villa (Mab. 1868): t. supra compressa, infra depresso-convexa, subcostulato-striata, albido-grisea, subseriatim maculis castaneis fulguratis marmorata, anfr. $5-5 \frac{1}{2}$, sat convexi, primi lente, cæteri rapide accrescentes, ultimus permagnus, compresso-rotundatus, obsolete angulatus, non descendens, apertura tate subrötundato-ovata, Iunata; d. $18--20$, a. $8-11 \mathrm{~mm}$. (Sardinia et Corsica.)

- var. magnettii CANTr. (1840): t. supra conoidea v. compresso-convexa, infra convexa, confertim striata, grisea v. albidolutescens, maculis nigris castaneisque marmorata, subtus $1-2$ fasciata, anfr. $5-5 \frac{1}{2}$, subdepresso-convexi, regulariter accrescentes, ultimus magnus, rotundatus, valde descendens, apert. ovatorotundata, lunata, peristomate incrassato, subexpanso; d. 20-22, a. 11-12 mm. (Gallia, Corsica, Sardinia.) Syn. H. serpentina Dup. t. 4. f. 4 .

- var. cenestinensis CR.. \& Dев. (1869): t. turbinatodepressa, vix striatula, albida, zonis spiralibus, latiusculis, regularibus, nigro-castaneis, albido-variegatis, cincta, anfr. $4^{1} / 2$, vix convexiusculi, ultimus paullo descendens, subtus bifasciatus, apert. late lunaris, intus livide albida, albolabiata, mar- 
ginibus distantibus, fere subparallelis; d. 19, a. $11 \mathrm{~mm}$. (Corsica.) Fig. Journ. Conch. t. 5. f. 7.

317. H. minoricensis Mitrre (1842): t. subdepressa, subtiliter striatula; anfr. celeriter accrescentes, ultimus peripheria rotundatus, deflexus; apertura transverse lunari-ovalis, intus carnea, margine columellari stricto; diam. 16, alt, $8-9 \mathrm{~mm}$. (MFinorca.) T. albida, fasciis 5 interruptis, punctisque castaneis ornata, anfr. 4, vix convexiusculi, peristoma patulum, carneo-labiatum, margine columellari dilatato, plano. "Persimilis speciminibus minoribus H. serpentinæ (Pisanis): differt anfractibus celerius accrescentibus, ultimo altiore, macula-umbilicali brunnea deficiente" (Pfr.):

318. H. melitensis Fér. (1822): t. depresso-globularis, sublævis; anfr. regulariter accrescentes, ultimus rotundatus, paullo deflexus; apertura lunato-elliptica, intus livida, peristomate albo, margine basali strieto; diam. 19, alt. $11 \mathrm{~mm}$. (Malta.) T. albida, unicolor vel maculis pallide fuscis subseriatis nubeculata, anfr. 4, planiusculi, peristoma reflexum, marginibus conniventibus.

319. H. scherzeri ZeL. ap. Prr. (1868): t. globoso-depressa, striatula; anfr. ultimus inflatus, profunde deflexus; apertura subcircularis, margine basali arcuato; diam. 20, alt. $11 \mathrm{~mm}$. (Hisp. ad Gibraltar.) T. albida, punctis raris corneis pellucidis conspersa, vel fasciis 5 continuis fuscis ornata, anfr. $t-4^{1 / 2}$, ennvexiusculi, peristoma albo labiatum, marginibus approximatis, callo junctis, breviter reflexis, columellari intus obsolete unidentato.

320. H. provincialis Ben, ap. Pfr. (1859): t. conoideodepressa, striatula; anfractus regulariter accrescentes, ultimus utrinque depressiusculus, peripheria obtuse angulatus, antice perdeflexus; apertura rotundato-lunaris, intus pallide carnea, margine basali calloso, strictiusculo, subdentato ; diam. $17-20$, alt. $81 / 2-11 \mathrm{~mm}$. (Sicilia.) T. coerulescenti- vel (corneo-) albida, unicolor, peristoma lahiatum, marginibus subconvergentibus, dextro expanso et revoluto, basali reflexo, umbilicum omnino vel partim clandente. Var. saracena Ben., t. fasciis 4 abruptis notata.

(H. strigata Fér. var.)

**) Testa umbilicata vel perforata.

321. H. recondita nov. sp.: t. semiobtecte umbilicata, subglobosa vel globoso-depressa, levigata vel transversim vix striatula, spiraliter plus minus lineata; apertura rotundato-lunaris, albolabiata, peristomate pallide brunneo, margine dextro vix expansiusculo, basali forte arcuato, reflexo; diam. 20, alt. 10-12 mm. 
(Ital. in prov. Napolitana.) 'T. lutescenti-alba, fascia pallide castanea inframediana et vestigiis fasciarum supremarum, anfr. 5-6, convexiusculi, sutura tenui, vel convexi, sutura immersa, ultimus rotundatus, subtumidus, antice valde descendens, apertura intus alba, marginibus approximatis, macula umbilicali fuscobrunnea. Variat minor, umbilico margine columellari reflexo interdum fere omnino clauso; d. 16 , a. $10 \mathrm{~mm}$. (In Aprutiis : Adami sp. ut H. signata Fér.)

322. H. carseolana FÉR. (1822): t. aperte perforata, globoso-depressa, irregulariter striata; apertura anguste ovalis, peristomate alba, margine basali strictiusculo; diam. $181 / 2$, alt. $81 / 2$ mm. (In Apcnninis romanis.) T. alba, fasciis $2-3$ evanescentibus, pallide castaneis, anfr. $4 \frac{1}{2}$, convexiusculi, ultimus descendens, peristoma patulum, sublabiatum, marginibus approximatis, basali subcalloso, solum prope insertionem fasciola fusca notato. (v. Mart.)

323. H. surrentina A. Schm. ap. Mart. (1858): t. subobtecte perforata, subdepressa, suhtiliter striata; apertura ovalis, peristomate fusco-corneo, margine basali arcuato; diam. 16, alt. $7 \mathrm{~mm}$. (Italia, prov. Napolitana.) T. ex carneo albida, fasciis 4 interruptis pallide castaneis, anfr. $4^{1 / 2}$, convexiusculi, ultimus descendens, peristoma patulo-reflexum, sublabiatum, rnarginibus approximatis, macula umbilicali fusca (v. Mart.); anfr. ult. utrinqne compressus, peripheria rotundatus, antice deflexus (spec. Mart.)

324. H. strigata (Müll.?) FÉr. (1822): t. anguste umbilicata, orbiculato-depressa, supra costata vel costato-plicata, apertura rotundato-ovalis, lunata, peristomate albo, margine basali arcuato; diam. 19, alt, $81 / 2-9 \mathrm{~mm}$. (Italia merid.) T. alba, superne fasciis tribus, medio una, ferrugineo-brunneis plus minus interruptis notata, dense regulariter costata, infra alba unicolor lævigata, spira paullisper turbinata, anfr. $3^{1 / 2}$, celeriter accrescentes, planiuseuli, ultimus deflexus, depressus, peripheria rotundatus, infra medio convexior, apertura intus paullo margaritacea, nitida, fasciis translucentibus, margine exteriore patulo, basali breve reflexo, columellari late reflexo, umbilicum semioccultante. Syn. $H$. posidaniensis Tib. (spec. ab Adami). Fig. Rossm. Icon, f. 227. - Rossir. tres formas hujus pervariabilis speciei depinxit: fuscolabiata Rossm. f. 684 (H. strig. v. sicula Ben., H. teriolensis Adami ex spec.): t. alba unicolor vel fasciis maculosis multimode varians, fauce et peristomate fusculis (Italia, Sicilia); 2) glctbrata Z., Rossm. f. 228, t. latius umbilicata, apertura magis rotundata, costis planioribus; 3) corrugata Z., Rossm. f. 229, t. superne acutius costulata, umbilico majore, marginibus peristomatis approximatis; d. 20, a. $91 / 2 \mathrm{~mm}$. (Ital.) Cel. Isseu ex Umbria sequentes variationes enumerat: 1) t. umbilico subclauso, carina nulla, costis numerosis et tenuibus, d. $16^{1} / 2$, a. $10 \mathrm{~mm} .:$ 2) t. umbilico aperto, carina nulla, costis numerosis et tenuibus, d. 17 , a. $10 \mathrm{~mm}$.; 3) t. umbilico aperto, carina 
nulla, costis rarioribus et tenuibus, d. 19 , a. $9 \mathrm{~mm}$. (var. glabrata Z.?); 4) t. umbilico aperto carina obtusa, costis crassioribus, d. 20, a. 10 mm. (var. corrugata?); 5) t. umbilico angusto, carina acuta, costis distantibus elevatis, d. 20, a. $10 \mathrm{~mm}$. (H. umbr. Ch.?)

- var. umbrica Cr. ap. Mas. (1868); t. mediocriter umbilicata, depressa, albido-lutescens, costis lamelliformibus ornata, anfr. 4-5, primi regulariter, ultimus celerrime accrescentes, ultimus maximus, depressus, infra convexus, ad peripheriam obscure carinatus, antice subito descendens, apertura lunata, ovata, vel subsecuriformis, magna, intus alba, marginibus approximatis, peristoma paullulum expansum, album, sublabiatum, ad umbilicum reflexum; d. $17--21$, a. $8 \mathrm{~mm}$. (Ital. in Umbria.)

\section{tf Testa carinata, depressa.}

325. H. scabriuscula DEsir. (1830): t. obtecte perforata, striato-rugosa, compresso-carinata; apertura lunato-securiformis, peristomate superne recto, marginibus approximatis; diam: 24, alt. $10 \mathrm{~mm}$. (Sicitia occid.) T. albida, sæpe fusco-maculata, depressa, sub lente densissime inter rugis spiraliter lineata, anfr. $4-4^{1}{ }_{2}$, paullulum exserti, subplani, ultimus planus vel juxta carinam elatam paullulum immersus, basi turgidus, antice deflexus, apertura intus fusca angulata, margine basali albo, sublabiato, arcuato, angulatim reflexo, columellari dilatato, appresso. Syn. H. erycina Jan 1832. Fig. Rossm. Icon. f. 23t-36. Variat spira omnino plana, spira paullo turbinata vel etiam subscalaridea. Var. 1) selinuntina Phil.(1836), t. minor, tenue striata, carina breviore, maculis rufis confluentibus; d. 18, a. $9 \mathrm{~mm}$; 2) major: d. 26, a. $10 \mathrm{~mm}$; 3) minor: d. 15, a. $6 \mathrm{~mm}$.

- var. segestana Pril. (1836): t. plus minus umbilicata, plicata, distinctius spiraliter lineata, acute carinata; anfr. ult. eonvexiusculus; d. $16-20$, a. $7-9$ mm. - Fig. Rossm. Icon. f. 233. Var. Irepanensis Huet ap. Pfr. (1856): t. spira subgradata, anfractus supra plani, scalariforme exserti, carinati, ultimus compresse-carinatus, infra convexus, tumidus; d. $15-16$, a. $8-8 \frac{1}{2} \mathrm{~mm}$. (Trepani et Marsala Siciliæ.)

326. H. malziana Parr. ap. Pfr. (1860): t. subobtecte umbilicata, arcuatim subruditer striata; anfr. mediani exserti carinati, ultimus subangulatus; apertura subovalis, peristomate superne expanso, marginibus continuis; diam. 28. alt. $13 \frac{1}{2} \mathrm{~mm}$. (Rhodus.) T. subdepressa, albida, fasciis interruptis fuscis ornata, spira subconoidea, anfr. ult. depressus, carina antrorsum sensim evanescente, antice deflexus, basi mon inflatus, apertura intus sordide carnea, peristoma album, marginibus callo crassiusculo junctis, columellari late reflexo. Syn. H. casareana rar. nana Mouss.? (Pfr.) 
(H. paciniana, tiberiana, muralis var.)

18. Helicogena (FÉr.) Risso. T. imperforata vel subobtecte perforata, globosa, plerumque fasciata, striata; anfi. 4-6, convexi, ultimus magnus, ventrosus, descendens; apertura lunata, orbicularis, peristoma patulum vet rectum, intus callo limbatum, margine columcllari reflexo.

Habitat in hortis, fruticetis, in truncis arborum frondosarum ad muros etc. Fossilis in strato pleistocenico medio et supero.

† Testa globoso-conica, imperforata, peristomate superne expanso, basali reflexo.

327. H. aspersa MüLL. (1774): t. conoideo--globosa, ruguloso-malleata; diam. 40, alt. $36 \mathrm{~mm}$. (Regio mediterranea, Britannia.) T. solidula, griseo-Iutescens, fasciis variis castaneis, flammulisque flavidis ornata, rugosa, quandoque irregulariter et sat tenuiter striata, anfi. 4-5, convexiusculi, ultimus perventrosus, maximam testæ partem efficiens, transversim dilatatus, antice deflexus, spira sapissime brevis, columella obliqua leviter arcuata, alba, apertura lunato ovalis, oblique verticalis, peristoma expansum, incrassatum, album, marginibus callo albescente et subpellucido in aucem dejecto, subjunctis, columellari dilatato. Syn. H. grisea et variegata Gmel.1789. H. hortensis Penn. 1776, H. lucorum Pult. 1799." Fig. Rossm. Icon. f. 294. Variat 1) globosa, 2) globoso-conoidea (scalaris, sinistrorsa), 3) tenuis, 4) solida, 5) minor (d. 25, a. $27 \mathrm{~mm}$.); colorem a flavescente ad fere nigrum, unicolorem vel fasciatam variat.

328. H. mazullii $\mathrm{J}_{\mathrm{AN}}$ (1832): t. globoso-conica, transversim costulato-rugosa; diam. 25-36, alt. 22-28 mm. (Sicilia, Italia merid.) T. tenuis, subpellucida, lutescens, sæpissime unicolor, inferius ventricosa, anfr. 4, convexi, ultimus magnus, inflatus, sursum attenuatus, apertura ampla, rotundato-subovata, parum lunata, peristomate vix subpatulo, albo-labiato, marginibus callo tenui, nitido, albescente, in faucem dejecto, conjunctis. Syn. H. crispata Costa 1829, $H$. retirugis Menke $1830, H$. rugosa Mhlf, ex Mke, H. costa Ben. Fig. Rossm. Icon. f. 295, 96. - Variat zonata Bg.: t. fasciis 5 fuscescentibus ornata. Celeberr. Bourguignat duas speries hujus stirpis distinxit: mazulli typ., t. globoso-conica, flavida, eleganter striata, anfr. 4, convexiusculis, celeriter crescentibus, ultimo magno, inflato, descendente, d. 28-35, a. 30-40 mm. (Fig. Rev. 1860. t. 6, f. 1-3) \& quinciacensis Maud. (1839), t. conica, sordide lutescens, rugoso-plicata, rugis elevatis, appressis, sæpe inter se reticulatis, anfr. 4, convexis, celeriter crescentibus, ultimo magno, rotundato, ad aperturam valde desceudente, d. 22-28, a. 25-35 mm. (Fig. Rev. Zool. 1860. t. 6, f. $4-6)$. 
ff $T$. subglobosa, inflata, temis, imperforata, peristomate undique recto.

32\%. H, aperta Bons. (1878): t. distanter et irregulariter plicato-striata, non decussata; diam. 20-26, alt. $21-30 \mathrm{~mm}$. (Europa merid.) T. pellucida, ovato-globosa, fuscescens vel rufescens, anfi. 4, convexiusculi, rapide accrescentes, ultimus peramplus testam fere totam efficiens, antice non descendens, apertura amplissima, acute obovalis, intus margaritacea, peristomate simplici, acuto, albolimbato. Syn. H. neritoides Chemn. 1780, H. naticoides Drp. 1801. Fig. Rossm. Icon. f. 285. Variat 1) castanea, 2) viridula.

330. H. tristis PFR. (1745): t. striata, lineis spiralibus obsolete decussata; diam. 23, alt. $23 \mathrm{~mm}$. (Corsica.) T. pellucida, ovato-globosa, olivaceo-fusca, rufo 5-fasciata, anfr. 4, convexiusculi, ultimus inflatus, antice leniter descendens, apertura lunato-ovalis, parum lumata, intus nitida, fasciis externis pellucentibus, peristoma simplex, rectum, margine columellari dilatato, reflexo, appresso. Fig. Kob.-Rossm. Icon. f. 1049.

tit Testa globosa, imperforata, lincis confertis spirali. bus magis minusve decussatu, peristomate superne recto, basali reflexo.

331. H. melanostoma Drp. (1801): t. obtecte perforata, globosa, spiraliter lineata, cinereo-carnea, subfasciata, peristomate et pariete aperturali nigro-fuscis; diam. 28-32, alt, 28-34 mm. (Gallia merid.) T. solida, ventrosa, irregulariter striata, anfr. 4-5, convexi, ultimus inflatus, columella obliqua, strictiuscula, apertura ampla, lunato-rotundata, intus pallide carnea, nitida, peristoma rectum, cum pariete intus purpurascenti-fuscum, subincrassatum, marginc dextro arcuato, columellari dilatato, diffuso. Fig. Rossm. Icon. f. 286, 576. Var. 1) vittate Rossm. (H. nucula Parr.), t. candida, fasciis obscuris (12345) notata, lineis spiralibus perdistinctis (Rossm. f. 577, 78); 2) candida Rossm., t. candida, unicolor, altior, plicato-striata (Rossm. f. 579).

332. H. figulina Parr. ap. Rossir. (1839): t. subolotecte perforata, conico-globosa, rugulosa, sub 5-fasciata; apertura acute ovato-lunaris, peristomate albo; diam. 22, alt. $20 \mathrm{~mm}$. (Grecia, Turcia, Rhodos.) T. ventricosa, sordide albida, faseiis 3 superioribus prope positis, inferioribus 2 remotis, anfi. 4, vix convexiusculi, peristoma rectum, incrassatum, margine columellari arcuato, dilatato, subappresso-reflexo. Fig. Rossm. Icon. f. 580 (forma minor). Bourg. Amèn. II. t. 21, f. 1, 2 (forma major). 
- var. pomacella PArr. ap. Moưss. (1854): t. rugulosostriata, corneo-albida, unicolor vel fasciis 5 fuscis (3 subobsoletis); apertura lunato-subcircularis, peristomate simplici recto; diam. 22, a. $20 \mathrm{~mm}$. (In utroque litore Bospori.) Fig. Bourg. Amèn. II. t. 21. f. 3,4 .

333. H. philibinensis Fruw. ap. Rossur. (1839): t. imperforata, depresso-globosa, nucleo mediocri, plicato-striata, 5 fasciata; apertura lunato-rotundata, peristomate albo; diam. 24, alt. 16 mm. (Rumelia, Macedonia.) T. sub lente decussata, alba, fasciis, fuscis, 3 superis angustis, 2 inferis remotis, latis, anfr. 4, convexiusculi, ultimus inflatus, apertura diagonalis, intus concolor, nitida peristoma simplex, rectum, margine columellari declivi, dilatato, appresso (H. philibensis Friw. teste Pfr., H. philippinensis Friw. ap. Kob. monente Rossm.). Fig. Rossm. Icon. f. 581.

334. H. obtusata Z. ap. ALB. (1850): t. obtecte perforata, depresse globosa, nucleo maximo, subtiliter elevato-striata, spiraliter lineata et minutim punctata, sordide flava, distanter 4 fasciata; apertura oblongo-rotundata, peristomate pallide hepatico; diam. 31, alt. $31 \mathrm{~mm}$. (Rossia merid., Tauria.) T. tenuis, diaphana, fasciis 4 castaneis vel rectius fasciis 5 , secunda et tertia pone jacentibus et sæpius in una confluentibus, anfr. 4, spira obtusissima, polita (anfr. primis duobus fortissime accrescentibus), apert. intus nitida, peristoma simplex, columella intrans, stricta, margine fornicatim reflexo, perforationem fere tegente. Syn. H. vulgaris Parr. ap. Rossm. 1830, H. obtusalis Mouss. 1854. Fig. Rossm. Icon. f. 582, Rev. Zool. 1860. t. 7. f. $4-6$. - Variat bicincta Dub., t. fasciis 1 et 5 ornata, ce. teris deficientibus.

- 335. H. cincta MüLL. (1774): t. obtecte perforata, ovatoglobosa, nucleo parvo, spiraliter lineata, fusco-grisea, fascia latissima fusca supra peripheriam et 2 angustis pallidioribus ad basin; peristoma intus castaneum; diam. 37-40, alt. $30-35 \mathrm{~mm}$. (Illyria, Ital. bor., Dalm., Gall. merid., Bulgaria, Thessalia.) T. subglobosa, solida, rugoso-striata, spira brevis, conica, (anfr. primis duobus lente accrescentibus)- anfr. 5, convexi, ultimus magnus, ventrosus, apertura rotundato-subovato-lunaris, intus plumbea, peristomate et pariete castaneis, margine columellari obliquo, strictiusculo, dilatato, subinerassato. Syn. $H$. grisea Pfr. olim, nec Lin., H. lemniscata Brum. Fig. Rossm. Icon. f. 287, 583, 584. Varict raro fasciis 5 distinctis vel fasciis. 5 confluentibus. Var. pollinii Da Campo, t. alba, epidermide tennissime lutescente decidua, peristomate candido (forma albina, H. lutescenti similis.) Cfr. H. melan. var. vittatam Rossm.

- ambigua PARR.: t. destincte 5-fasciata. ad aperturam albida, apertura intus alba, peristoma intus obscure castanea, mar- 
gine columellari subverticali, regulariter arcuato, basali profundius demerso. (Corfu \& Epirus.) Syn. H. cyrtotena Bourg. 1860. Variat (in Cephulonia) minor et calcarea (var. boreatis Mouss.)

336. H. ligata MüLt. (1774): t. subobtecte perforata, distincte spiraliter lineata, rufo 5 fasciata; peristoma album, margine columellari arcuato, obliquo, macula umbilicali alba; diam. 35- -36 , alt. $36-37 \mathrm{~mm}$. (Italia media et merid.) T. conico-globosa, irregulariter striata, reticulata, fulvescenti-albida, fasciis 3 superioribus, anfr. 5, convexi, ultimus ventricosus, vix descendens, apertura rotundato-lunaris, intus alba, nitida, peristoma subsimplex. Syn. H. gussoneana Sh. ap. Pfr. 1846. Fig. Rossm. Icon. f. 288, 290, 1011 -43. Variat 1) alba unicolor (teste celeb. Kob. $=$ H. aimophila Bourg. Rev. Zool. 1869. t. 20. f. 14-16: testa diam. 25, alt. $23 \mathrm{~mm}$, in Aprutiis); 2) albescens Jan, t. albida, fascia suprema et infima distinctis, intermediis maculose dissolutis.

- var. pomatella Tiв.: t. minor, globosa, distinctissime quadrifasciata (1. - 3, 4, 5). Italia merid. Fig. Kob.- Rossm. Icon. f. 1040 .

-_ var. calabrica Ков. (1875): t. lineis spiralibus obsoletis, fasciis 5 rufescentibus angustis, columella et pariete aperturali rufescentibus; d. 34, a. $32 \mathrm{~mm}$. (Calabria.) Fig. Kob.-Rossm. Icon. f. 1037 .

- var. pratutia Тıв. (1869): t. subrimata, depresso-globosa, valide costulato-rugosa, albida, fasciis 3 latis pallide fulvescentibus, apertura semiovata; margine columellari dilatato; d. 39 , a. 37 mm. (In Aprutiis.) Fig. Bull. mal. ital. 1869. t. 3. f. 12, 13 (Kob.Rossm. Icon. f. 1041-42).

- var. compana Tiв. (1869): t. imperforata, elevato-globosa, obsolete striata, læte citrina, fascis (5-) 6 castaneis ornata, apertura ovato-lunaris, margine columellari subdilatata; cl. 26 , a. $25 \mathrm{~mm}$. (Campania.) Fig. Bull. mal. ital. 1869. t. 3. f. 1, 2 (Kob.- Rossm. Icon, f, 10t3.)

337. H. secernenda Rossı.: t. imperforata, non spiraliter lineata, fasciis 5 angustis, subrqualibus notata; peristoma album vel pallide brunneum, margine columellari strictiusculo, parum obliquo, macula umbilicali pallide rufo-brunnea; diam. 37-40, alt. 42 mm. (Dalm., Bosna, Herzegovina.) T. turbinato-globosa; fusco-albida, fasciis 3 superioribus spadiceis, $2 \& 3$ interdum subcon- 
fluentibus, 2 inferioribus, anfr. $4-5$, convexiusculi, ultimus inflatus, columella subcarnea, apertura lunato-ratundata, intus tenue plumbea, pellucide fasciata, columella superne depressa. Syn. H. ligata Pfr. Fig. Rossm. Icon. f. 289.

338. H. lutescens Z, ap. Rossir. (1837), t. obtecte perforata, tenuissime et densissime spiraliter lineata, albo-lutescens, raro dilute fusculo-fasciata; peristoma album vel pallide hepaticum, margine columellari subverticali, leviter arcuato; diam. 30, alt. $32 \mathrm{~mm}$. (Transsilv., Serbia, Gallicia, Vollınia, Bessarabia.) T. striatula, turbinato-globosa, unicolor vel raro fasciis 5 angustis sæpius obsoletis ornata, spira brevis, conoidea, anfr. 4, convexiusculi, ultimus inflatus, columella alba, apertura ovato-rotundata, lunata, intus margaritacea, margine columellari superne dilatato, fornicatoreflexo. Syn. $H$. cinerascens Andrz. Fig. Rossm. Icon. f. 292.

339. H. lucorum MüLL. (1774): t. subobtecte perforata, pertenue spiraliter lineata, latissime castaneo-fasciata (anfr. ult. medio albo-cingulato, utrinque fascia obscura latissima) et distanter strigata; peristoma late obscure castaneum, margine columellari substricto, perobliquo; diam. 44, alt. 40-42 mm. (Italia, Turcia, Rossia merid.) T. globosa, substriata, albida, castaneo late fasciata et remote strigata, anfr. 5, convexiusculi, ultimus inflatus, antice breviter descendens, apertura oblique ovato-lunata, intus nitida, pallida vel fasciata, peristoma obtusatum, simplex, marginibus callo tenuissimo junctis, exteriore arcuato, ad medium aufr. penultimi affixo, columellari substricto, superne dilatato, reflexo, perforationem fere tegente. Fig. Rossm. Icon, f. 291.

- var. straminca Brig. (1825): t. major, globosa v. conica, magis ventrosa, fasciis $2-4$ castaneis cingulata, anfractibus lentius accrescentibus, ultimo et penultimo ventricosis et globulosis, apertura magna, altiore quam lata, lunato-rotundata; d. 53 , a. $50 \mathrm{~mm}$. (In Aprutiis.) Fig. Bourg. in Rev. Zool. 1860. t. 4. f. 3, 4.

- var. castanca Oriv. (1801): t. imperforata, depressoglobosa, zonulis 2-3- vel 4 uniformiter castaneis vel fusco-nigris cingulata, anfr. 6, convexiusculi, regulariter crescentes, ultimus parum inflatus, antice lente ac valde descendens, apertura lunatosubtetragona, parvula, intus coeruleo-candida, peristomate rufobrunneo, simplici, margine exteriore pone basin anfr. penultimi affixo, columellari incrassato, perdilatato, magis horizontali; d. 39, a. $40 \mathrm{~mm}$. (Constantinopolis.) syn. H. mahometana Bourg. 1860 , Fig. Rev. Zool. 1860, t. 4, f. 5, 6 . 
- var. onixiomicra Boukg. (1866): t. semiobtecte-perforata, conico-globosa, lutescenti-albida, zonis duabus, fasciis nigrescentibus passim interruptis, cincta, anfr. $6 \frac{1}{2}-7$, convexi, regulariter crescentes, apertura parvula, obliqua, lunato-oblonga, intus candida, peristomate rufobrumneo, simplici; d. 42 , a. $38 \mathrm{~mm}$. (Montenegro.) Fig. Rev. Zool. 1860, t. 3. f. 1, 2.

- var. rumclica Mouss. (1863): t. magna, turbinato-elevata, ventricosa, alba, castaneo transversim striata, fasciis pallide rufis latissimis sapius duabus, anfr. $6 \frac{1}{2}$, supremi convexiusculi, ultimi 2 convexi, ultimus parum inflatus, antice lente ac valde descendens, apertura oblique ovato-lunata, intus candida, peristoma rufo-brunneum, simplex, margine exteriore pone basin anfr. penultimi affixo, marginibus subparallelis; d. 42 , a. $48 \mathrm{~mm}$, apert. alt. 27, diam. int. $21 \mathrm{~mm}$. (Rumelia.) - Obs. Hæc et precedentes 2 varietates speciem distinctam orientalem his characteribus notatam forte efficient: testa distinctius decussata, anfr. ultimus parum inflatus, antice valde descendens, apertura parva, intus candida, margine exteriore fere ad basin anfractus penultimi affixo \&c.

340. H. taurica KRxN. (1833): t. obtecte perforata, spiraliter lineata, lutescenti-albida, strigis fusco-nigris irregulariter radiata; anfr. medii subfasciati; peristoma hepaticum, margine columellari perdilatato, fornicatim reflexo; diam. 52, alt. $48 \mathrm{~mm}$. (Tauria.) T. globosa, ventrosa, strigis latioribus et angustis, pallidis et obscure castaneis inæqualiter munita, oblique striata, ad suturam plicata, spira conoidea, anfr. 5, convexiusculi, ultimus magnus, apertura ampla, rotundato-lunata, intus albida, peristoma subpatulum, subsimplex. Syn. H. radiata (radiosa) Z. ap. Rossm. Fig. Rossm. Icon. f. 456. - Variat sæe minor.

341. H. socia Rossir. ap. Prr. (1853): t. subumbilicata, depresso-globosa, pallide fulva, fasciis latis interruptis saturate castaneis, ornata; anfr. ultimus peripheria albo-cingulatus; diam. 43, alt. $26 \mathrm{~mm}$. (Constantinopolis.) T. tenuiuscula, oblique striata, anfr. $5^{1 / 2}$, convexiusculi, ultimus rotundatus, antice vix descendens, basi brunneo-radiatus, apertura diagonalis, rotundato-lunarta, peristoma subsimplex, margine supero fere recto, infero breviter reflexo, versus umbilicum perdilatato, fere adnato. Variat major, alba, epidermide fugace lutea partim obducta. (Pfr.)

342. H. pomatia LiN. (1758): t. semiobtecte perforata, spiraliter lineata, rufescens vel flavescens, fasciis fuscis obsoletis or- 
nata; peristoma carneum vel rufescens, margine columellari leviter arcuato, subverticali, superne dilatato, reflexo; diam. 37, alt. 40-42 mm. (Europáa.) T. globosa, ventricosa, rugoso-striata, anfr. 5, convexiusculi, ultimus magnus, subcompressus, apertura ampla, lunato-ovalis, intus nitida, albida, peristoma patulum, subincrassatum. Syn. Pomatia antiquorum Leach. Fig. Rossm. Icon. f. 1, 2. - Variat 1) t. magis depressa, tenui, apertura ovali (H. rustica Hn.), 2) t. magis globơsa vel conoidea, crassiore, pallidiore, fasciis suturatis, apertura magis rotundata (H. gesneri Hn.); 3) albina; 4) minor:d. 26, a. $27-31 \mathrm{~mm}$. (Transsilv.)

- var. sch7äflii Mouss. (1859): differt testa ventroso-globosa, obtecte perforata, spira depresso-conoidea, nucleo albo, anfr. mediis spiraliter lineatis, apertura magis trausversali, intus griseoalba, peristomate et pariete fusco-griseis; "d. 50, a. $47 \mathrm{~mm}$." (Epirus, Serbia.)

Subfam. Pupina. Corporis pars posterior a pede distincta, spiraliter contorta et testa restita.

Testa oblonga, cylindracea vel fusiformis, apertura longitudinalis.

\section{Gen. Buliminus Ehrenb.}

Animal Helicum incole simile, tortili elongutiore et tentaculis inferioribus brevioribus.

Testa perforata, oralis, oblonga vel ovato-conica; apertura longitudinalis, dimidium longitudinem non superans, plerumque non atlingens, ovata, peristomate plerumque expanso, numquam contimu, marginibus incqualibus; columella stricta, lavis, basi minime truncata.

Syn. Buliminus Ehrenberg 18:31. - Bulimus (Scopoli 1777) Aucct. mult. pr. p.

Obs. Helix sect. Cochlicella Alb. ab auctoribus pluribus ad hoc genus ducitur.

1. Zebrina Hesd. Testa solida, oblongo-conica rel fusiformi-cylindrica, alba (sape fusco strigata); anfractus 7-11, ultimus spira brevior: apertura paria, obtique ovalis; peristoma rectum, intus labiatum, rarius dentatum. 
IIabitat locis apricis soli expositis, in cespitibus gramineis et sepibus vivis, ad rupes murosque per regiones orientales mediterraneas (una tantum species in Europa media.) Fossilis solum in strato pleistocenico supero.

+ Apertura peristomate incrassato vel sublabiato.

*) Testa dextrorsa.

1. B. detritus MÜLL. (1774): t. rimato-perforata, ovato-conica, alba, unicolor v. fusco-strigata; anfr. 7-8, convexiusculi, penultimus antepenultimo fere duplo latior; ultimus $2 / 5$ longitudinis æquans, apertura marginibus disjunctis; long. 20, diam. 9-10 mm., apert. 9-10 mm. longa. (Europa meridionalis et media.) T. solida, nitidula, tenue et irregulariter striata, sæpe et præsertim superne tenue spiraliter lineata, in nedio ventricosa, spira conica, obtusa, anfractus ultimi rapide accrescentes, ult. spira paullo brevior, sutura immersa, apertura acute ovalis, paullo lunata, intus luteo-carneola, peristoma rectum, albo-sublabiatum, margine columellari dilatato, reflexo, perforationem rimatam infra sat latam non tegente. Syn. Helix scpium et detritus Gmel. 1788, B. ratiatus Brug. 1789, Hel.turbincte Oliv. 1791. Fig. Rossm. Icon. f. 42,390. Variat 1) albinus, unicolor; 2) radicutus Pfr., Ch., t. alba, corneo-vel brunneo-strigata; 3) unicolor Jan, t. cornea, peristomate intus et sutura albis, raro aljostrigata; 4) Tuteus Z., t. lutea, fuscn-radiata; 5 )major, elongata;6) minor, abbreviata; 7) tumidus Parr., t. medio ventricosior (Epirus, Constantinop.) - Cfr. B. subdetritus Bourg.

2. B. fasciolatus Ourv. (1801): t. subrimata, ovali-oblonga, albida, fusco-strigata; anfr. $7-8$, planulati, ultimus $1 / 3$ longitudinis subrquans, penultimus antepenultimo 1/3 latior; apertura intus et paries castaneæ, marginibus disjunctis; long. 20, diam. $7 \frac{1}{2} \mathrm{~mm}$., apert. $7 \mathrm{~mm}$. longa. (Tauria, Creta, Rhodus.) T. solida, striatula, sub lente spiraliter lineata, nitida, spira turrito-conica, obtusa, anfr. ultimus basi attenuatus, sutura superficialis, columella brevis subrecta, apertura acute ovata, subtriangularis, peristoma simplex, album, margine exteriore levissime patulo, columellari breviter reflexo, appresso, perforationem fere tegente. Fig. Rossm. Icon. f. 910, 911. Variat 1) unicolor, candidus; 2) minor: 1. 15, d. 6-7 mm.; 3) "sordide albus, punetis et lineis fulminatis minutis corneis dense aspersa" (Pfr.)

3. B. illibatus Z. ap. Rossu. (1837): t. rimata, ovali-oblonga, alba; anfr. $7-7^{1} / 2$, vix convexiusculi, ultimus $2 / 5$ longitudinis subrequans; apertura columella flexuosi, callosa; long. 15, diam. 6 mm., apert. $5-5 \mathrm{~mm}$. longa. (T'auria.) T. solida, levigata vel tenuissime striatula, alba, nitida, spira attenuata, obtusa, anfr. ultimus 
vestigio carinæ perobtusæ, apertura oblique semiovata, parum altior quam lata, intus brunnescenti-fulvida, peristoma vix patulum, sublabiatum, columella flexuosa, callosa, (convenienter Limn, peregræ, vulgari \&c.) Fig. Rossm. Icon, f. 381. (Rossm.)

4. B. dardanus Friw. ap. Phis. (1842): t. subperforatorimata, fusiformi-turrita, alba; anfr. $8-9$, convexiusculi, penultimus antepenultimo $1 / 3$ longior; ultimus ${ }^{1 / 3}$ longitudinis fere æquans; apertura marginibus subdisjunctis; long. 26, diam. 9 mm., apert. $9 \mathrm{~mm}$. longa. (Tauria, Constantinop.) T. striatula, alba, quandoque superius fusculo-strigata, ab anfractu penultimo tumidulo sursum turriforme attenuata, anfr. ult. deorsum attenuatus, sutura impressa, apert. acute ovata, intus dilute fuscula, peristoma patulum, remotius labiatum, margine columellari late reflexo, columella strictiuscula. Fig. Rossm. Icon. f. 905, 906. Variat major: 1. 30, d. $11 \mathrm{~mm}$.

5. B. cylindricus MKe (1830): t. rimata, cylindrica, alba, sipe fusco-strigata; anfr. $9-10$, planiusculi, penultimus antepenultimo vix $1 / 4$ longior; ultimus $1 / 3$ longitudinis fere æquans; apertura marginibus callo junctis; long. 24-25, diam. $8 \mathrm{~mm}$, apert. $8 \mathrm{~mm}$. longa. (Tauria.) T. solida, striatula, superne pertenue spiraliter lineata, alba, concolor vel transverse fusco-strigata, anfr. ultimus basi juxta rimam profundam subcompressus, apertura acute ovata, intus flavido cornea, peristoma rectum, albo-sublabiatum, margine columellari subincrassato, patente, dilatato, callo parietali sæpe sat valido. Syn. B. tauricus Lang ap. Pfr. 18 18 , Chondrus lincatus Kryn. 1832, B. lin. Rossm. 1837. Fig. Rossm. Icon. f. 380. Variat 1) turgidus Kryn. (1833), t. abbreviata, obesa, cylindraceo-conica; 1. 18-20, d. 7 mim.; 2) fuscilabrus Kryn. (1833), t. minor, alba, turgidula, nitida; 3) fusiformis Mke (1830), t. major, anfr. 11, strigis obliquis cinereo-fuscis, pellucidis; 1. 33, d. $8 \mathrm{~mm}$., ap. $9 \mathrm{~mm}$. longa; 4) obsoletus Parr. ap. Mouss. (1863), t. transversim striata, striis spiralibus obsoletis decussata, supra interdum subgranulata, griseo-alba, tenuiter radiata (Tauria, Parr. ex.); 5) bettai Ch. (1837), t. gracilior, magis elongata, rima latiore, apertura angustior, peristoma minus incrassatum, callo marginis jungente multo tenuiore; 1. 26 , d. $7 \mathrm{~mm}$.

6. B. varnensis FrIW. ap. PFr. (1847): to rimata, fusiformioblonga, lactea; anfr. 10, convexiusculi, penultimus et antepenultimus æquales, ultimus $3 / 10$ longitudinis subæquans; apertura parva, marginibus callo junctis; long. 21, diam. $6^{1 / 3} \mathrm{~mm}$. (Turcia prope Varna et Constantinopolin.) T. solida longa, gracilis, irregulariter striata, nitida, lactea, nnicolor, anfractus ultimus ceteris paullo ventricosior, juxta rimam subcompressus, aperiura oblongo-ovata, intus flavida, peristoma subrectum, acutum, intus albo-labiatum. columellari dilatato, patente. Variat major: 1. 25, d. $7 \mathrm{~mm}$. 
7. B. subulatus Rossı. (1837): t. minutissime rimata, attemuato-cylindrica, cinerea, fusculo-strigata; anfr. 8 convexiusculi, ultimus ${ }^{2} / 7$ longitudinis subśequans; long. 10, diam. $3 \mathrm{~mm}$., apert. $2^{1 / 2} \mathrm{~mm}$. longa, ex icone. (Tauria: Kob.) T. olstusa, apertura acute semiovata, peristoma rectum, subsimplex, margine exteriore curvato longiore. Fig. Rossm. Icon. f. 393. ( $12 \mathrm{~m}$.)

(B. subdetritus Bourg.)

**) Testa sinistrorsa.

8. B. gibber KrYN. (1833): t. ovato-oblonga, paullulum rugoso-striatula; anfr. 8 , vix convexiusculi, ultimus $1 / 3$ longitudinis vix superans; peristoma subrectum; long. 20 , diam. $7 \frac{1}{2} \mathrm{~mm}$. (Tauria.) T. perforato-rimata, ventricosa, obtusiuscula, interdum striata, sub lente tenue spiraliter lineata, coerulescenti-albida, spira conico-turrita, sutura impressa, submarginata, apertura rotundatoovalis, peristoma subsimplex, marginilus approximatis, columellari dilatato, angulatim reflexo. Syn. B. revolutus Z. ap. Rossm. 1837. Fig. Rossm. Ycon. f. 389. Variat obsolete lutescenti-radiatus.

9. B. phorcus Bourg. (1859): t. conico, cylindracen, eleganter striatula; anfr. $81 / 2$, convexi, ultimus $1 / 3$ longitudinis requans; peristoma undique expansum; long. 25, diam. $8 \mathrm{~mm}$. (Tartria.) T. profunde perforato-rimata, nitens, albida, acutiuscula, anfr. regulariter crescentes, ultimus basi subrotundatus, apertura lunato-rotundata, nitida, peristoma candidum, margine columellari paullulum dilatato, marginibus approximatis. (Bg.)

\section{ti Apertura dentata. \\ *) Testa dextrorsa.}

10. B. subdetritus BourG. (1855): t. conoideo-ovata, ventricosa; apertura lata, truncato-ovata, columella basi obsoleto plicata, marginibus callo ad insertionem tuberculifero junctis; long. 14-15, diam. $7 \mathrm{~mm}$., ap. 6/3 mm. longa. (Turcia ad Varna, Eidos et Constantinopolin.) T. rimato-perforata, griseo-albida, opaca, obsolete obscure strigata, subirregulariter striata, spira convexo-conica, anfr. 7, convexiusculi, ultimus $2 / 4$ longitudinis subæquans, basi sulbcompiessus, columella substricta, peristoma incrassatum, margine exteriore brevissime reflexo, columellari lato, patente. Fig. Bourg. Amén. I. t. 12 . f. $5-8$.

11. B. bidens KRYN. (1833): t. cylindrica, ruguloso-striata; apertura semiovata; columella flexuosa, callosa, prominula, dentem simulans; palatum in fundo valide et oblique uniplicatum; long. 17. diam. $4 \frac{2}{3}$ mm., ap. $4 \mathrm{~mm}$. longa. (Tauria, Turcia.) T. rima ta, alba unicolor vel fusco-strigata, anfr. 9-10, planiusculi, lentissime 
accrescentes, ultimus $1 / 3$ longitudinis vix superans, basi angulato-compressus, peristoma subrectum, albolabiatum. Syn- $B$. clansiliaformis Menke 1830, Brephulus fuscostrigatus, zebrinus et clausiliaformis Bk 1837. Fig. Rossm. Icon. f. 382, 383. Variat 1) gracilis, albus (B. appendiculatus Jan); 2) ventricosior', fusco-strigatus (B. zebriolatus Z.); 3) ventricosior, minor, unicolor (Z. perdiceus Z. p. p.); 4) minor: 1. 12, d. $2 \frac{3}{3} \mathrm{~mm}$., ap. $3 \mathrm{~mm}$. longa (Ch. attenuatus Kryn.)

12. B. spoliatus PArr. ap. PFr. (1846): to ovato-cylindracea, striatula; apertura ovata, dente parietis lamelliformi, margine exteriore medio unidentato; long. 101/2, diam. $3^{1 / 2} \mathrm{~mm}$. (Gracia.) T. rimata, solidula, nitida, cinerea, unicolor vel fusco-strigata, anfr. 8 , planiusculi, peristoma subpatulum, intus labiatum, marginibus callo junctis, columellari brevi, reflexo.

- fusiformis (Desh.?) Br: t. major, candida, interdum fuscostrigata, sæpe apice nigricans; 1. 14, p. $4^{1 / 4} \mathrm{~mm}$. longa. (Ins. Thermia; Horea?)

13. B. zebra OLiv. (1801): t. fusiformi-cylindracea, fuscostrigata, lævigata, nitida; apertura angusta, semiovalis, trisinuatocoarctata: lamella 1 parietali, plica 1 columellari, tuberculo dentiforme in margine exteriore; long. 14-15, diam. $31 / 2-4 \mathrm{~mm}$. (Gracia, Rumelia, Rhodus, Cerigo.) T. rimata, obtusiuscula, anfr. 9, planiusculi, ultimus $1 / 4$ longitudinis subæquans, ceteris convexior, peristōna patulum, intus labiatum, marginibus callo connexis. Syn. Helix zebriola et zebrula Fér. 1821. Fig. Rossm. Icon. f. 653. Variat 1) albus, unicolor (B. calcareus Parr. ap. Anton 1839); 2) obesa: 1. 12, d. $4 \mathrm{~mm}$.

- tantalus PARr: t. apertura basi dilatata, subquadrangulari. (Dardanell.)

14. B. compactus Friw. ap. PFr. (1848): t. ovato-oblonga, obsolete strigata, opaca; apertura truncato-ovalis, tridentata: lamella 1 in pariete, dente parvulo in margine dextro et in colus mella; long. $4 \frac{1}{2}$, d. fere $6 \mathrm{~mm}$., ap. $5 \mathrm{~mm}$. longa. (Creta.) T. profunde rimata vel perforata, solida, alba, obsolete fusculo-strigata, spira sursum attenuata, obtusa, anfr. 9-10, convexiusculi, ultimus $1 / 3$ longitudinis subæquans, peristoma vix expansiusculum, intus sublabiatum, marg. colum. angusto, patente.

15. B. rothi PFr. (1855): t. ovato-oblonga, striatula; apertura truncato-ovalis, subquadidentata: denticulo minimo juxta insertionem labri, secundo profundo-in ventre anfractus penultimi, tertio validiore transverso ad columellam, quarto maximo in margine dextro; long. $91 / 2$, diam. $3^{2} / 3$ mm., ap. $3 \frac{1}{2} \mathrm{~mm}$. longa. 
(Gracia.) T. breviter rimata, solidula, corneo et albido irregulariter strigata, spira elongata, in conum obtusulum terminata, sutura impressa, anfr. 8, convexiusculi, ult. basi rotundatus, antice obsolete scrobiculatus, apertura subverticalis, peristoma album vix expansiusculum. Syn. B. bergeri Pfr. p. p. 18t8. Variat t. graciliore, sulcylindrica, $10^{1 / 2} \mathrm{~mm}$. longa, $3 \mathrm{~m}$. m. lata.

\section{**) T'esta sinistrorsa.}

16. B. tournefortianus FÉR. (1821): t. magna, fusiformis; anfr. 11, planulati; apertura trisinuato-coarctata, margine dextro et sinistro unidentatis, dente tertio lamelliformi intrante in pariete aperturali; long. $25-27$ dian. $7 \mathrm{~mm}$., ap. $7 \mathrm{~mm}$. longa. (Turcia.) T. rimata, cretacea, unicolor, anfr. lente accrescentes, ultimus $2 / 7$ longitudinis vix superans, apertura intus fulva, peristoma patulum, intus labiatum, marginibus callo junctis. Syn. Pupa tridentata Lam. ap. Desh. 1836, Clausilia lavis Blainv. 1825. Fig. Rossm. Icon. f. 652.

2. Chondrula Веск. Testa solida, ovato-oblonga vel orata, conica, nitidula, striata, apice acmimata; anfr. 7-9; apertura semiovalis, dentata; peristoma albo-labiatum.

Habitat locis apricis, in cespitibus gramineis sub lapidibus etc. precipue in Europa meridionali-orientali (duo tantum species transalpinæ.) Fossilis in strato pleistocenico.

\section{† Testa dextrorsa.}

17. B. bicallosus Frrw. ap. PFr. (1847): t. fusiformi-cylindracea, spira a medio sensim attenuata; anfi. 10-11, vix convexiusculi; sutura submarginata; apertura coarctata 8 -formis, columella plica transversa valida dentiformi munita, margine dextro unidentato; long. 20-21, diam. $5 \mathrm{~mm}$, ap. $5 \mathrm{~mm}$. longa. (Turcia, ad Slivno, Eidos, Constantinop.) T. subperforata, tenuis, diaphana, subtilissime striatula, nitidula, pallide cornea, spira obtusa, anfr. ultimus $1 / 4$ longitudinis paullo superans, peristoma expansum, albo-labiatum, marginibus callo tenui junctis, dextro extus non scrobiculato. Syn. H. raymondi Bourg. 1853. Fig. Rossm. Icon. f. 908.

18. B. athensis Friw. ap. PFr. (1847): t. oblonga, sursum attenuata; anfr. 8-9, plani; sutura albo-marginata; apertura subrhombeo-semiovalis, in pariete tuberculo munita; long. 20, diam. $61 / 2$ mm., ap. $61 / 2 \mathrm{~mm}$. longa. (Girecia, mons Athos.) T. rimatoperforata, striatula, nitidula, cornea, anfr. ultimus $1 / 3$ longitudinis subxquans, peristoma expansum, albo-labiatum, marginibus callo crassiusculo, juxta insertionem marginis dextri in tuberculum transversum producto, junctis, columellari dilatato, patente. (Pfr.) 
19. B. pupa Brug (Auct.): t. ovato-oblonga, spira conica; anfr. 7, planiusculi; sutura submarginata; apertura semiovata;-in pariete unidentata; long. 17, diam. $7 \mathrm{~mm}$., ap. $7 \mathrm{~mm}$. longa. (Sicil., Grccia.) T. rimata, subtiliter striata, sordide lutescens, spira acutiuscula, anfr. ult. $2 / 5$ longitudinis subæquans, apertura obliqua, peristoma albo-labiatum, marginibus callo juxta angulum superum dentifero junctis, dextro expanso, columellari late reflexo. Syn. (Helix pupa Lin. 1758 teste Hanley = B. detritus Müll.), B. tuberculatus Turt. 1824 in Zool. Journ. II, B. emarginatus Desh. 1836, B. babauchii Ant. 1839, Pupa primitiva Menke 1830, B. kindermanni Parr. teste Jick. Fig. Rossm. Icon. f: 379 (719). Variat 1) grandis Mouss. (1859), t. najor, long. 17-18, diam. $7^{1 / 2} \mathrm{~mm}$., margine dextro in tertia supera paullo inflexo, intus densiori, tuberculo parietali transverse elongato (Cephalonia); 2) minor, ovatus, long. $9^{2 / 3}-10^{1 / 2}$, diam. $4^{1 / 4}-5^{1 / 2}$ mm., ap. $4^{1 / 2} \mathrm{~mm}$. longa.

- carneolus Z.: t. formæ parvæ sequentis persimilis, sed apertura subverticalis, tubereulo parietali distincto, et anfractus ult. dimidian testre partem attingens. (Constantinop.).

20. B. reversalis Bielz (1853): t. dextrorsa et sinistrorsa, cylindraceo-oblonga vel ovata; anfr. 7-8, priores valde, ulteriores minus convexi; sutura impressa; apertura semiovata, pariete vix unidentato; long. 11 , diam. $32 / 3$ mm., ap. $31 / 3 \mathrm{~mm}$. longa. (Transilv.) T. rimata, oblique striata, luteo-fusca, nitidula, apice attenuato, obtuso, anfr. ult. 1/2 altitudinis numquam attingens, peristoma patulum, albolabiatum, marginibus subparallelis, callo tenui supra subtuberculifero subconnexis, columella obliqua, subcallosa. Fig. Rossm. Icon. f. 930-934. Variat 1) dextrorsa: grandis Bz. to elongato-ovata, 1. $17^{1} / 2-26^{1} / 2$ d. $77 / 10-82 / 5$ mm.; relictus $\mathrm{Bz}$., t. minor, elongatior, l. 12-17, d. $5 \frac{1}{2}-6_{1}^{1} / 2 \mathrm{~mm}$.; tenuis Bz., t. gracilis, cylindracen, 1. $11-15^{1 / 2}$, d. $4^{1 / 1} / 2-51 / 2$ mm.; curtus Bz., t. brevis, abbreviata, 1. 11-13, d. $5^{1 / 2}-6 \mathrm{~mm}$.; parvus $\mathrm{Bz}$., t. elongata, rix ventricosula, 1. 10-11, d. $34 / 5-4^{2} / 5$ mm.; 2) sinistrorsa: major Bz., t. magna, ventricosa, l. $171 / 2-22$, d. $74 / 10-84 / 5 \mathrm{~mm}$. (B. venerabilis Parr.); minor $\mathrm{Bz}$., t. minor, elongatior, 1. 12-17, d. 51/2-61/2 mm. (B. conjunctus Parr.); elongatus Bz., t. pergracilis, cylindracea, 1. 11-15, d. $4^{1} \frac{1}{2}-5^{1} / 2 \mathrm{~mm}$.; brevis Bz., t. parva, brevis, 1. 11-13, d. 51/2-6 mm.; alpestris Bz., t. minuta, elongata-ovata, cylindracea, $1.10-11$, d. $3^{4} / 5-4^{2} / 5 \mathrm{~mm}$.; crassitabris Rm. f. 933, t. minor, peristomate crasse labiato (Vallachia minor.)

21. B. kutschigi Küst. (1845): t. cylindraceo-conica; anfr. 7 , leviter convexi; apertura verticalis, semiovalis, in pariete denticulo munito angulari et secundo majore profundius posito $\mathrm{mu}-$ nita; long. $7 \frac{1}{2}$, diam. $3 \mathrm{~mm}$. ap. $2 \frac{2}{3} \mathrm{~mm}$. (Dalmatia merid.) T. rimata, solidula, subtilissime striata, nitidula, carneolutescens, spira 
sursum sensim attenuata, anfr. ult. basi tuberculato-gibbus, apertura denticulo minuta ar insertionen marginis dextri et secundo in ventre, peristoma albo-labiatum, incrassatum, margine dextro obtuso, sul)sinuato, columellari brevi, juxta rimam reflexo.

\section{**) Apertura 3-7 dentata.}

22. B. microtragus PARR. «p. Rossur. (1839): t. ovato-oblonga; anfr. ult. $2 / 5$ longitudinis subæquans; apertura semiovalis, coarctata, quadrisinuata, tridentata, dente parietali verticali, columellari et marginis dextri oppositis; long. 12, diam. $5 \frac{1}{3} \mathrm{~mm}$., ap. $4 \mathrm{~mm}$. longa. (Gracia, Bulgaria, Rumelia, Dalmatia infer.) T. arcuato-rimata, substriata, apice brevi, conico, anfr. 7, planiusculi, nltimus basi compressus, peristoma callosum, subreflexum, semper callo basali protuberante inter dentes inferiores oppositos, marginibus utrinque callo dentiformi approximatis, dente columellari unico et dextro 1 majore, latis, transverse positis, hoc superne bi-tridenticulato, paries medio dente compresso intrante munita. Syn. B. labidens Parr. Fig. Rossm. Icon. f. 651. Viriat minor, 1. 81/2, d. $4 \mathrm{~mm}$., apertura callo parietali dentiformi sinistro obsoleto.

23. B. tridens MüLL. (1774): t. oblonga v. oblongo-ovata; anfi: ult. $1 / 3$ longitudinis subæquans; apertura semiovalis, subtrisinuata, tridentata, dente parietali obliquo et dente marginis exterioris suboppositis; long. 12, diam. 5 mm., ap. 4 mm. longa. (Germ., Helv., Gall., Austria, Carinth., Carniolia de. in fere tota Eur. merid.) T. rimata, subtiliter striata, cornea, pellucescens, solida, anfr. $6-8$, convexiusculi, ultimus ad aperturam flavidus, sutura sat impressa, columella brevis, recta, peristoma carneo- vel albo-labiatum, vix expansum, marginibus callo tenuissimo ad dextrum tubereulifero junctis, columellari ad basin et externo superne dentibus brevibus obtusis, hoc in summo denticulis 1-2 munito. Syn. Pupa tridentata Brard 1815, B. variedentatus Hartm. 1815. Turbo quadridens v. Alt. Fig. Rossm. Icon. f. 33, 305, 720 (var.). Variat 1) elongatus (Pupa eylindrus Mus. Berol. ol.); 2) eximius: 1. 12-16, d. $5^{1 / 2} \mathrm{~mm}$. (Illyria, 'Transsilv., Sard., Corsica?-B. tr. var. eximia Rm. f. 305, 732, Torquilla spreta Villa, Ch. elatus Andrz. teste Krryn.); 3) minor 1. 8-10 mm., d. $4 \mathrm{~mm} . ; 4$ ) unidentatus Iss. (1866), t. fulva, diaphana, apertura dente parietali tantum munita; 1. 10, d. $4^{1 / 1} / 2 \mathrm{~mm}$. (Pisa); 5) caucasicus Mouss. (1863). t. paullo major, crassior, obtusior, apertura latior, dentibus, præsertim columellari unico, fortioribus (Podolia ad Smotrica et ad Sebastopol.) (Foss.)

24. B. quinquedentatus BorN (1780): t. subcylindrica; anfr. ult. $1 / 3$ longitudinis vix superans; apertura semiovata, coarctata, bisinuata, 5-6 dentata, dente parietali obliquo et dente marginis exterioris perapproximatis, oppositis; long. 17-19, diam. 6 
mm., ap. 6 mm. longa. (Datmatia, Triest, Bosna.) T. rimata, subtiliter striata, acuminata, corneo-lutescens, ad aperturam transversim alba, solida, anfr. 8, planiusculi, apertura dentibus 5-6 coareta: 2 in pariete ( 1 magno, lamelliforme, intrante in medio, superiore punctiformi), 2 in margine dextro illis oppositis (1 elato, valido, perobliquo, superiore punctiformi), 2 profundis obtusis in columella (columella contorta dentem superiorem formante), peristoma albolaliatum, expansum, marginibus callo tenui junctis. Syn. Ch. quinqueplicutus Jan 1832, Ch. major Kiryn. Fig. Rossm. Icon. f. 30t. Variat tumidus, l. 13 , d. $5 \frac{2}{3} \mathrm{~mm}$.

25. B. albolimbatns PFr. (1848): t. ovato-oblonga; anfr. ult. $2 / 5$ longitudinis subæquans; apertura subsemiovalis, basi subingulata, dentata: dente 1 angulari, 1 complanato, intrante in pariete, 1 parvulo ad basin columellæ, 2 parvulis profundis in parte supera marginis dextri; long. 14 , diam. vix. $6 \mathrm{~mm} .$, ap. $51 / 2$ mm. longa. (Saratow, Somchetia.) T. rimata, solida, striatula, nitidà, corneo-castanea, spira elongato, obtusa, sutura levis, peristoma vix expansiusculum, intus callosum, extus albido-limbatum. Syn. Pupa obesa Parr. (Pfr.)

26. B. septemdentatus Rотн (1839): t. ovato-oblonga; anfi. ult. $2 / 3$ longitudinis vix superans; apertura truncato-ovalis, sinuoso-coarctata, 7 dentata: dentibus 3 in pariete, summo angulari minimo, 1 horizontali in columella, 2 profundis in margine dextro, septimo subbasali; long. 12 , diam. $4^{2} / 3$ mm., ap. $4^{1 / 2} \mathrm{~mm}$. longa. (Oenussa, ins. prope Scios, Grocia: Roth teste Kst; Syria.) T. rimata, solidula, carneo-fuscula, spira sursum in conum attenuata, anfr. 7, summi convexi, sequentes subplani, ult. basi subcompressus, peristoma expansiusculum, intus carneo-callosum, marginibus subparallelis. Variat minor, ventricosior, $1.9^{1 / 3}, \mathrm{~d} .4^{2 /} \mathrm{mm}$.

27. B. bergeri Roth (1839): t. fusiformi-ovata; anfr. ult. ${ }^{1 / 3}$ longitudinis paullo superans; apertura semiovalis, coarctato-sinuosa, marginibus callo, superne in dentem incrassato junctis, tridentata: 1 parietali, profundo, 1 horizontali columellari, 1 conico in margine exteriore; long. $14-15^{1} / 2$ diam. $4^{2} / 3-6 \mathrm{~mm}$., ap. $5-5^{2} / 3 \mathrm{~mm}$. longa. (Gracia, Thessatia, Euboca.) T. solida, subtiliter striata, spira superne albomarginata, anfr. 9, vix convexiusculi, apert. dentibus omnibus validis, albis, callosis, peristoma albolabiatum, margine dextro subrecto, medio antrorsum dilatato, basali et columellari reflexis. Syn. Pupa tridentata Ant. 1832, P. tricuspis Rossm. 1812, B. euboicus Reeve 1819, B. mühlfeldti Pfr. 1819. Fig. Rossm. Icon, f. 721. Variat minor, I. 10, त. $4 \mathrm{~mm}$. 
28. B. levaillantianus Bourg. (1855): t. acuminato-ovata; anfr. ult. $2 / 5$ longitudinis subrquans; apertura 4 dentata: 1 flexuose linguæformi intrante in angulo parietis, 1 transverso ad basin columellæ, 1 minuto basali, 1 crasso in margine dextro; long. 9-10, diam. $4 \frac{1}{2}-5 \mathrm{~mm}$. , ap. $33 / 4-4 \mathrm{~mm}$. longa. (Turcia, prope Adrianopolim.) T. solida, striatula, albido-cornea, spira ventrosula, apice conicn, anfr. 7, convexiusculi, peristoma albolabiatum, margine dextro prope insertionem profunde sinuato, columellari lato, patente. Fig. Bourg. Amèn. I. t. 13. f. 10-13.

\section{it Testa sinistrorsa. \\ *) Apertura dentata.}

29. B. orientalis Friw, ap. PFr. (1848): t. fusiformi-cylindracea; anfr. 10, planiusculi; apertura 3 dentata: 1 columellari, valida, perobliqua, plicæformi, 2 in margine sinistro; long. 17, diam. $5 \mathrm{~mm}$., ap. $5 \mathrm{~mm}$. longa. (Ins. Prinkipos.) T', subperforata, striatula, corneo-lutescens, anfr. ult. $2 / 7$ longitndinis subæquans, apertura oblonga, ringens, sinuosa, peristoma rubello-labiatum, margo exterier callo tenui denticulifero cum margine columellari tortuosa brevissimo conjunctus. Fig. Rossm. Icon. f. 909.

30. B. niso Risso (1826): t. ovato-oblonga; anfr. 9-10, plani; apertura trisinuata, 3 dentata: 1 parietali, 1 obliquo elongato ad basin columellæe, 1 parvo conico intus in margine sinistro; long. 12, diam. $5 \mathrm{~mm}$, ap. $4^{1 / 2} \mathrm{~mm}$. longa. (Croatia, Dalmatia, Italia, Gall. merid.) T. rimata, nitida, fusco-cornea, sutura marginata, anfr. ult. ceteris paullo convexior, $1 / 3$ longitudinis subrequans, apert. semiovalis, denticulo angulari sæpe deficiente in pariete munita, peristoma subrectum, labiatum, callosum. Syn. B. seductilis Z. ap. Rossm. 1837. Fig. Rossm. Icon. f. 306, 724. Variat 1) cylindricus Rossm. f. 307, elongatus, 1. 12, d. 4 mm.; 2) minor.

31. B. quadridens MIÜLt. (1774): t. ovato-oblonga; anfr. 7, planiusculi; apertura cruciatim sinuata, 4 dentata 1 parietali, 1 in margine exteriore, 2 columellaribus minoribus; long. 10, diam. $31 / 2$ mm., ap. $3^{1} / 3$ longa. (Gallia, Helv., Tirolia, Germ. occid., Ital., Hisp.. Dalm.) T. vix rimata, lævigata, lutescenti-cornea, anfr. ult. $1 / 3$ longitudinis subæquans, basi compressus, apert. semiovalis, peristoma labiatum, patulum. Syn. Jaminia heterostropha Risso 1826. Fig. liossm. Icon. f. 308, 723. Variat 1) major: 1. 12-14, d. 4 mm.; 2) elongatus, t. cylindracea; 3) minor: $1.5^{1 / 4}-6$, d. $2^{1 / 2}-3 \mathrm{~mm}$.; 4) tridentatus: $t$. dente inferiore columellari (in antecedentibus majore) deficiente (Pyren.); 5) loewii Phil. (1844), t. major, magis cylindrica (Attica). Rossm. f. 928 . 
32. B. bolterianus Phic. (1845): t. ovato-oblonga; anfr. 7, convexi; apertura parvula, dente parvulo, compresso, profunde in pariete; long. 9, diam. $4^{1} / 3$ mm., ap. $3 \mathrm{~mm}$. longa. (Dalmatia.) T. profunde rimata, striatula, carneo-albida, nitida, anfr. ult. 1/3 longitudinis subæquans, basi gibbus, apert. oblique semiovata, peristoma subrectum, crasse labiatum, marginibus callo junctis.

\section{**) Apertura edentata.}

33. B. chersonesicus Sow. ap. JAY (1839): t. ovato-oblonga; anfr. 8 , convexi; apert. ovato-oblonga, intus pallide aurantiacx; long. 21, diam. $9 \mathrm{~mm}$. (Tauria.). T. rimata, subobscure rugosostriata, coerulescenti-albida, fulvescente hinc illine pallide tincta, spira conico-turrita, peristoma acutum, paullulum expansum, simplex. (Bg.)

3. Napæus Albers. Testa ovato-oblonga, striata, brunnea; anfr. 6-9, convexiusculi, ultimus spira brevior; apertura rotundato-ovalis; peristoma expansum, intus labiutum, margine columellari scepe dilatato.

Ifabitat locis montosis, in rupibus et truncis folisque arborum frondosarum, sæpius in quercinis et fagetis humosis, in fruticetis vel rarius sub lapidibus, præcipue terrarum orientalium. Fossilis in strato pleistocenico.

34. B. pusio Broder. (1836): t. ventroso-ovata, irregulariter striata, olivaceo-falva; peristoma albo-labiatum, marginibus callo junctis, dextro breviter expanso, columellari subverticali, dilatato, patenti; long. 12, diam. $7 \mathrm{~mm}$. (Syra.) T. compresse umbilicata, tenuis, nitida, spira convexo-conica, anfr. 6 , comvexiusculi, ultimus spira vix brevior. Syn. B. delesserti Bourg. 1852, B. gastrum Mouss. 1854. Fig. Bourg. Cat. rais. t. 2. f. 1-3.

35. B. etuberculatus FrauenF. (1867): t. oblonga, subventroso-conica, subruditer striata, fusca; peristoma expansum, albolabiatum, marginibus callo tenuissimo sed lato albo junctis; long. 13, diam. $54 / 5$, ap. long. $4^{3} / 5 \mathrm{~mm}$. (Syra.) T. umbilicata, diaphana, anfr. 7, convexiusculi, "sensim accrescentes, ult. dorso spira paullo brevior, ap. $1 / 3$ testæ paullo superans, margine dextro parum curvato.

36. B. friwaldskyi PFr. (1847): t. subcylindrica, striatula, fusco-cornea; apertura elongata, subsemiovalis; peristoma breviter expansum, valide albolabiatum, marginibus subparallelis, sejunctis, dextro repando, columellari reflexo, appresso; long. 14 , diam. $4^{1} / 2$ mm., ap. long. $4^{1} / 3$ mm. (Ins. Prinkipos.) T. subimperforata, sur- 
sum attenuata, obtusa, anfr. 9, subplani, ult. 1/3 longitudinis non requans.

- var. rayneratianus RArm. (1855): t. fülvo-cornea, anfi. 10, convexiusculi, angustiores, ult. $2 / 7$ long. formans, apertura oblonga, peristoma valide labiatum, margine dextro vix expanso, intus supra medium subdentato; long. 16, diam. $4^{2} / 3 \mathrm{~mm}$. $(\mathrm{G}$ (l) lipoli.) Fig. Journ. Conch. 1855. t. 15. f. 5-9, Bourg. Amèn. I. t. 8. f. $5-9$.

37. B. montanus Drap. (1801): to ovato-conica, fusea, striata et minute granulata; apertura late ovata; peristoma albosublabiatum, expansum; long. 15, diam. $6 \mathrm{~mm}$, ap. $5 \frac{1}{2} \mathrm{~mm}$. longa. (Europa mertia.) T. rimata, obtusa, lineis densis spiralibus tenuissimis ornata, anfr. 7, convexiusculi, ultimus $3 / 5$ longitudinis subrequans, sutura marginata, sat impressa, columella areuata, peristoma margine columellari reflexo, dextro parum longiore. Syn. H. lackhamensis Mont. 1803, H. buccinata Alten 1812, B. montacuti Jeffr. 1830, H. sylvestris Stud., IIartm., H. obscura Schrank 1803. Fig. Rossm. Icon. f. 4. Variat 1) major: Rossm. (f. 386), t. elongato-turrita, anfr. $8-9$, sat convexi; 1. 18-21, d. $7 \mathrm{~mm}$., ap. 6-7 mm. longa (Laibach, München, Gallia); 2) minor, 1. 12, d. $5 \mathrm{~mm}$. ; 3) coloratus Colb., t. brunnea, peristomate subpurpurascente (Belg.) (Foss.)

- collini Micr. (1831): t. ovato-conica, transversim regulariter striata, densissime granulata, anfr. 7 (5-6: Mich.), subplani, ultimus convexus, apertura angustior, oblonga-ovata, columella stricta; 1. $12^{1} / 2$, d. $5 \frac{1}{3}-5^{2} / 3 \mathrm{~mm}$., ap. $5 \mathrm{~mm}$. longa. (Gall.) Fig. Micl. Compl. t. 15. f. 41 .

38. B. monticola Rotri (1856): turrito-oblonga, vix striatula, corneo-fuscula; apertura ovalis; peristoma albolabiatum, late reflexum, marginibus approximatis, callo junctis; long. $15-20^{1} / 2$, diam. $7 \mathrm{~mm}$., ap. 6-7 longa. (Grecia, mons Parnassus.) T. nitidula, spira elongata, obtusa, sutura levis, albo-filosa, anfr. $7 \frac{1}{2}-8$, planulati, ultimus $1 / 3$ longitudinis vix superans. Fig. Mal. B1. 1856. f. 1. f. 4,5 .

39. B. rupestris KRYN. (1833): t. ovato-turrita, parum striata-carneo-fuscula; apert, ovalis; peristoma reflexiusculum, albosublabiatum, marginibus conniventibus; long. 20, diam. $6 \mathrm{~mm}$., ap. $6 \mathrm{~mm}$. longa. (Tauria; Apennin.?). T. tenera, nitidula, sutura inferne albida, anfr. $7-8$, convexiusculi, ultimus precipue basi versus perconvexus, peristoma margine dextro forte arcuato, superne incurvato, columellari multo breviore, strictiuscuIo. Syn. B. apenninus Jan. 1830, B. assimitis Z. ap. Rossm. 1837. Fig. Rossm. Icon. f. 685. Pracipue peristomate et anfractibus ab antecedente distinguitur. 
40. B. tener Z. ap. Rossur. (1837): t. ex anfr. ultimo subgloboso ovato-turrita, substriata, tenera, carneo-lutescens; apertura rotundato-ovalis; peristoma simplex, marginibus fere contiguis, macula alba callosa terminatis, dextro valde arcuato; long. 13, diam. $5 \frac{1}{2}$ mm., ap. $4^{1 / 2} \mathrm{~mm}$. longa. (Tauria.) T. perforato-rimata, anfr. 7-8, convexiusculi, ult: $1 / 3$ long. vix æquans, perist. margine colum. reflexiuseulo. Syn. Peristoma merduenianum Kryn. 1833, B. fragilis Parr. ap. Ant. 1839. Fig. Rossm. Icon. f. 388.

41. B, caucasicus PFr. (1852): t. oblongo-turrita, tenuis, ruguloso-striata, lineis impressis confertissimis sub lente sculpta, luteo-cornea; apert. semiovalis; perist. albolabiatum, margine dextro subrepando, expanso, columellari superne perdilatato; long. 18, diam. 7 mm., ap. $8 \mathrm{~mm}$. longa. (Tauria: Dubois teste Mouss.) T. subperforata, spira convexo-turrita, sutura levis, submarginata, anfr. 7 , planiusculi, ultimus $3 / 7$ longit. subæquans, basi rotundatus, columella subplicata, recedens, perist. tenue Fig. Chem. Cab., Bul. t. 36. f. 14,15 .

42. B. humberti Bourg. (1857): t. cylindrica, striatula, cornea; apertura parva, semiovata; peristoma albolabiatum, non reflexum, acutum; columella recta; long. 19, diam. $4 \mathrm{~mm}$. (Sebastopolis.) T. perforata, anfr. 8, convexi, regulariter crescentes, sutura impressa. Fig. Rev. Zool. 1857, t. 2. f. 5-7.

43. B. cefalonicus Mouss. (1859): t. oblongo-conica, irregulariter rugoso-striata, albida, striis et maculis impressis corneis interrupta; apertura lunato-ovalis; peristoma vix patulum, late albolabiatum; long. 16, diam. $6 \mathrm{~mm}$. (Ins. Cefalonica, Bosna, Serbia.) T. rimata, anfr. 7, convexiusculi, ult. 1/3 longitudinis æquans, apert. intus grisea, columella arcuata, peristoma marginibus callo tenui albido junctis, columellari breviter reflexo, versus dextrum incurvato.

44. B. cretensis Pfr. ap. PhiL. (1845): t. cylindraceo-turrita; striata, carneo-lutescens; apertura semiovata; perist. expansiusculum, valide albo-labiatum, marginibus callo tenui, dextrorsum obsolete tuberculifero, junctis; long. 18-21, diam. $8 \mathrm{~mm}$., ap. $7^{1 / 2} \mathrm{~mm}$. longa. (Candia.) T. solidiuscula, spira elongata, sutura levis, albomarginata, anfr. 7--8, planiusculi, ultimus $1 / 3$ longitudinis æquans, apertura intus alba, nitida, margine columellari reflexo, subappresso.

45. B. gastrum Ehrenb. (1831): t. ovato-oblonga, minute striata, cornea; apertura semiovalis; peristoma album, simplex, 
margine dextro expanso, columellari dilatato, libere reflexo; long. $13 \frac{1}{2}-15$, diam. $7 \mathrm{~mm}$., ap. $6 \mathrm{~mm}$. longa. (Ins. Syra.) T. tenuis, nitida, sutura linearis, albo-marginata, anfr. 6, vix convexiusculi, ultimus spira paullo brevior, columella brevis, strictus.

46. B. subtilis Rosss. (1837): t. cylindracea, substriata, cornea; apertura acute ovalis; peristoma subrectum, margine exteriore multo longiore, albolabiato; long. 14 , diam. $4^{3} / 4$ mm., ap. $4 \mathrm{~mm}$. longa. (Dalmatia.) T. apice attenuata, nitida, anfr. 9, vix convexiusculi, lentissime accrescentes, ultimus $2 / 7$ longitudinis subæquans, apertura cervice tumida, albocineta. Syn. B. noctivagus Parr. Fig. Rossm. Icon. f. 302. Variat minor, $9 \mathrm{~mm}$. non superans, paullo gracilior, pallidior, anfractibus 9-10 (var. corcyrensis Mouss. 1859). Ins. Corfu.

47. B. olivaceus Pfr. ap. Phil. (1845): t. oblonga, striatula, virenti-cornea; apertura semiovata; peristoma breviter expansum, valide albolabiatum, marginibus callo tenissimo junctis; long. 13, diam. $5 \mathrm{~mm}$., ap. $5 \mathrm{~mm}$. longa. (Candia.) T. sericina, anfr. 6, convexinsculi, ult. $1 / 3$ longit. paullo superans, columella verticalis, medio subplicata.

48. B. græcus BECK (1837): t. ovato-oblonga, striatula, carneo-flavida; apertura ovato-oblonga; peristoma subsimplex, margine exteriore recto, columellari reflexo, ambobus callo pertenui junctis; long. 16-18, diam. $7 \mathrm{~mm}$. (MIorca.) T. nitida, anfr. $7^{\mathrm{t}} / 2$, convexiusculi, sutura superficialis, irregulariter subcrenulata, albescens, apertura $2 / 5$ longitudinis æquans, Syn. corneus Desh. 1836. Fig. Mal. Mal. Bl. XX. t. 2. f. 2. ("Long. 20, diam. $10 \mathrm{~mm}$." Dsh.)

49. B. obscurus MIüLL. (1774): t. ovato-oblonga, striatula, fusca; apertura rotundato-ovata; peristoma albo-sublabiatum, expansum, margine columellari brevi, dilatato; long. 7-10, diam. 4-41/2 mm. (Europa.) T. nitidula, brunneo-fusca, pellucida, acutiuscula, anfr. 7, convexiusculi, lente accrescentes, ultimus 1/3 longitudinis vix æquans. Syn. Turbo rupium DaC. 1778, B. hordeaceus Brug. 1789, Hel. stagnonum Pult. 1799. Fig. Rossm. Icon. f. 387.

- var. oblongus WeSTERL. (1871): t. oblonga, anfr. tardissime accrescentes, penultimus præcedentem subæquans, sutura antice non ascendens, apertura parva, oblonga, fissura umbilicalis minutissima; 1. 11, d. $81 / 2 \mathrm{~mm}$. (Suecia.)

- var. asticriames 'Dup. 1849): t. conoiden, levigata, apertura ovata, peristoma patulum, planulatum, albo-fusculo labiatum, anfr. 6 , convexi, sutura profunda separati, ultimus mediam testre 
partem efformans; 1. 5-6, d. 2 mm. (Gallia, ins. St.-Marguerite, Belgia ad Namur.) Fig. Dup. Iist. t. 15. f. 7.

4. Petræus Albers. Testa oblongo-conica vel cylindraceo-oblonga, subito attenuata, cornea, albida, unicolor; anfr. 6-8, planulati, ultimus spira brevior; apertura ovalis, columella plus minusve distincte plicata; peristoma late expansum rel reflexum, marginibus approximatis, callo junctis.

Habitat in desertis lapidosis asiaticis, duæ tantum species incolæ Europæ sunt. Fossitis modo in strato oligocenico et miocenico infero.

50. B. niveus Parr. ap. Rossm. (1859): t. attenuato-cylindracea; apertura truncato-subrotundata, margine exteriore labiato, supra obsolete tuberculifero; alt. 23 , lat. $5 \mathrm{~mm}$. (Tauria.) T. alba, striatula, apice conico, obtusato, anfr. 8, convexiusculi, ultimus striatus, basi compressiusculus, apert. ${ }_{1 / 3}^{1 / 3}$ altitudinis nondum xquans, peristoma patulum, columella remota, strictiuscula. Syn. B. turbatus Parr. ap. Mouss. Fig. Rossm. Icon. f. 916.

51. B. cosensis ReEve: t. oblongo-turrita; apertura truncato-ovalis, peristomate subsimplici; long. 15, diam. $5 \mathrm{~mm}$., ap. $5 \mathrm{~mm}$. longa. (Ins. Cos Archipelagi græci.) T. coerulescenti-alba, substriata, nitida, spira turrita, obtusa, anfr. 7, summi convexi, sequentes subplani, ultimus $1 / 3$ longitudinis vix attingens, basi rotundatus, columella leviter arcuata, peristoma marginibus vix conniventibus, dextro breviter expanso, columellari reflexo.

\section{Gen. Cochlicopa (Fér.) Risso.}

Animal eidem generis Helicis simile, sed organis generationis simplicioribus; maxilla parum arcuata, striis verticalibus subtilibus cxarata, margine concavo vix denticulato; dentes radule subquadrati, in series transiersales dispositi.

Testa parvula, imperforata, ovato-oblonga, ovato-elliptica vel bulimiformis, nitida v. splendida, pellucida, epidermide destituta; anfr. 6-7, ultimus rotundatus; sutura marginata; apertura ovata, $1 / 3-1 / 2$ longitudinis civeiter aquans; columella brevis, arcuata, plus minusve truncata, peristoma rectum, subincrassatum. 
Syn. Butimus Auct. prise. - Achatina Aucr. - Cochlicopa Risso 1826. - Cionella JEFFr. 1830.

1. Zua Leach. Testa ovato-oblonga, lavis, splendida, fusco-cornca; anfractus convexiuscuti; apertura ovata, dimidium longitudinis non attingens, columella vix truncata, peristoma obtusum, marginibus callo junctis.

Habitat in umbrosis et humidis silvis, inter folia putrida, in muscis et truncis putridis, sub lapidibus, in pratis graminosis aquosis \&c. Fossilis in str. eocenico supero, oligocenico, miocenico infero, pliocenico medio et supero, nec non in str. pleistocenico.

1. C. lubrica MüLI. (1774): t. ovato-oblonga, fulvo-cornea; anfr. 5-6, convexiusculi; apertura ovali-elliptica, peristoma albido-carnescente-labiatum; long. 6-61/2, diam. $21 / 2-3 \mathrm{~mm}$. (Europa.) T. lævigata, lubrica, pellucida, anfr. lente accrescentes, ultimus $2 / 5$ longitudinis subæquans, basi rotundatus, columella leviter arcuata, subcallosa, basi introrsum obsolete truncata, apertura marginibus inæqualibus, callo vix perspicuo unitis. Syn. Turbo muscorum Ström 1765? Helix subcylindrica Linné 1767. Fig. Rossm. Icon. f. 43. Variat 1) nitens Kob. ap. Gall. (1852), t. major nitidissima, corneocastanea, anfr. $6 \frac{1}{2}$, columella distincte truncata, long. 7-8, diam. 3 mm. (Illyria, Scandin. bor.); 2) ovata Jeffr. (1862), t. multo minor, ovata, spira brevi (Britan., Suec.); 3) polita Parr. (in sched.), t. oblonga, flavescenti-cornea, pallida, polita, a pert. pyriformis, albolabiata, pariete lamina tenuissima opaca obtecta, 1. $6^{1} / 2$, d. $2 \frac{1}{2} \mathrm{~mm}$. (Græcia); 4) collina Drouët (1855), t. parvula, ovato-oblonga, virenti- vel rubescenti-fusca, anfr. 5-6, vix convexinsculi, apert. pyriformis, perist. subinerassatum, callo albido, margine colum. vix incrassato, 1. 3-4, d. 11/2-2 mm. (Gāll., Brit., Germ., Suec.); 5) minima Siem. (1847), t. gracilis, elongata, cylindracea, rubescenti-fusca vel albido-cornea, subliyalina, anfr. 5, apert. angusta, albido-labiata, $1.4-5, \mathrm{~d} .1^{1 /} / 2-2 \mathrm{~mm}$. (Bul. lubr. pulchellus Hartm. 1821, Ach. lubr. exigua Ilke 1830, H. lubricella Z. 1830, nomina, Gl. lubr. nilssoni Malm 1851, A. lubricoides Pot. \& Nich. 1838.)

- columna Cr. (1857): t. columelliformis, turrito-elongata, anfr. 6, lentissime accrescentes, ultimus $1 / 3$ longitudinis paullo superans, apert. angusta, elongato-acute ovata, sursum attenuata, pariete perobliqua; 1. 5-61/2: d. $1^{4} / 5-21 / 4 \mathrm{~mm}$. (Rossia ad Oka, Succia ad Borgholm, Germ. ad Augsburg et Blaubeuren.) Fig. Cless. in Jahr. mal. Ges. 18975, t. 2. f. 4, Exk. Fauna p. 184.

- pfeifferi WernL.(1874): t. oblongo-acuminata, subcylindrica, fulvo-cornea, anfr. 7, convexiusculi, apert. ovalis, supra et 
infra paullulum acuminata, $1 / 3$ longitudinis vix rquans, peristoma acutum, nullo modo incrassatum, columella vix truncata; 1.10 , d. $2 \mathrm{~mm}$. (Wï̈tem乙. prope Hohen-Wittlingen.) Fig. Weinl. Weichth. fauna d. schw. Alb. t. 4. f. 4. Monstrositas??

2. Azeca Licicr. Testa orato-clliptica, lavigata, splendida, fusco-cornea; anfr. planiusculi, ultimus a dorso compressus; apert. semirotundata, parva, 1/3 longitudinis aquans, dentata, vel edentula et marginibus callo filiformi extus tuberculifero junctis, columella compressa, callosa, peristoma incrassatum:

Habitat in silvis umbrosis montosis, inter folia dejecta et putrescentia, sub lapidibus, in muscis ad radices arborum etc. Fossitis in strato miocenico, pliocenico medio, et pleistocenico medio et supero.

1.) Azecastrum Boung.: testa apertura dentata, columella valida, basi truncatc.

2. C. tridens Pult. (1799): t. ovali-elliptica, spira brevi obtusa; apertura oblique pyriformis: paries infra callum unidenticulatus, medio plica longa intrante superne sinitrorsum arcuata et in columellam descendente munitus; columella in lamellam compressa, basi truncata, transverse unidentata; margo exterior medio unidentatus; palatum 2-denticulatum; long. 6, diam. $21 / 2 \mathrm{~mm}$. (Gallia, Belgia, Britan., German. oce., Austr. pr.d.) T. carneofulva, splendida, anfr. 7-8, planiusculi, rapide et irregulariter accrescentes, ultimus $1 / 3$ longitudinis subæquans, ad aperturam callo concolori transverso ornatus, sutura superficialis, apertura angustata, superne ad dextrum sinuata, margine exteriore producto, strictiusculo, superne sublunato, subacuto, dente incrassato-labiato. Syn. Carych. menkeanum C. Pfr. 1821, Hel. goodalli Fér. 1822, Car. politum Jeffr. 1830, A. matoni Turt. 1831. Fig. Rossm. Icon. f. 654. Veriat 1) crystallina, hyalina; 2) palatum 3-denticulatum.

- var. noulctiana Dup. (1849): apertura dentibus in fauce omnino destituta, denticulo marginis exterioris minore. (Gall. subpyrence; Britan.: spec. a clar. Jeffx.) Fig. Dup. Hist. t. 14. f. 12.

- var. alzenensis ST. Srm. (1870): t. ferrugineo-cornea, apertura ut in forma typica, sed dentibus duobus punctiformibus in fauce remotissimis et duobus peristomalibus, supero valido acuto, infero minuto remoto; 1. 6, d. 21/2 mm. (Gall. merid., mons Alzen 
et Lourdes.) Fig. A. tridens « ap) M. Tand. Hist. t. 22, f. 7-13, teste clar. S:t Sim. - Sp. orig. el. Fagot benigne misit.

3. 0. mabilliana FАGот in sched. (1876): differt a præcedente presertim testa majore, corneo-castanea, spira longiore, acutiuscula, anfr. regulariter lenteque crescentibus, apertura margine exteriore supra dentem fortius lunato-arcuato, dentibus et laminis validioribus, dente marginis laminiformi transverso cum dente unico palatali flexuose adhærente; long. $7 \frac{1}{2}$, diam. $3 \mathrm{~mm}$. (Pyrencec orient. ad Lourdes).

4. C. trigonostoma Bourg. ap. FAGot (1876): t. oblongoovoidea, leviter globuloidea; apertura lunato-trigonalîs, plica mediana parietali validissima, intrante, antice in arcum curvata, altera punctiformi, submarginali, plica columellari supera remotissima, altera crassa basali, plica palatali unica, parvula; long. 6, diam. $3 \mathrm{~mm}$. (Pyrence orient., prope Luchon.) T. superne inforneque attenuata, flavo-cornea, anfr. 7, vix convexiusculi, sat rapido crescentes, ultimus amplus, ad basin compresso-subangulato, plica palatalis in margine externo sita, cum lamella parietali mediana exacte opposita, peristoma margine externo superne leviter lunato, columella recta, marginibus callo albido tuberculifero junctis.

5. C. bourguignati FAGOT (1876): t. elongata; apertura exigua, lunata, oblique subtrigonati-pyriformis, plica mediana parietali mediocri, intrante, antice in arcum curvata; altera punctiformi, marginali, cum callo juneta, plica columellari unica basali, plicis palatalibus nullis; long. $7^{1} / 2$, diam. $3 \mathrm{~mm}$. (Gallia merid., prope Bouilly.) T. fragilis, cornea, spira attenuats, anfr. 81/2, planulati, lente accrescentes, ultimus vix major, $1 / 3$ altitudinis vix æquans, apertura minute plicata, marginibus callo albido tubereulifero junctis.

\section{2.) Hypnophila Bourg.: testa apertura edentula, columella basi indistincte truncata.}

6. C. pupæformis CaNTr. (1836): t. elliptico-ovata; anfr. 7, planulati, ultim. $1 / 3$ long. subæquans; apertura semiovalis, columella basi profunde dentato-truncata, margine dextro recto, infra medium obsolete unidentato; long. 7, diam. $3 \mathrm{~mm}$. (Dalm.Gracia.) T. carneo-fulvo, spleudida, apert. obliqua, margine dextro infra medium tenue labiata. Syn. Mastus canthrani Beck 1837, A. dentiens Rossm. 1839. Fig. Rossm. Icon. f. 630, Bourg. Amèn. II. 14. f. :-6.

7. C. zacynthia Rоти (1855): t. ovato-oblonga; anfr. 6, subplanati, ultim. penultimum xquans, $1 / 3$ long. formans; apertura 
exigua, rotundato-ovalis, columella profunde dentato-truncata, peristomate albo-calloso, reflexiusculo; long. $5 \frac{1}{4}$, diam. $2 \frac{1}{2} \mathrm{~mm}$. (Gracia, ins. Zante.) T. nitida, corneo-fulva, spira inflato-ccnica, sutura levis, apert. parum obliqua. Fig. Bourg. 1. c. f. 13, 14.

8. C. integra Moriss. (1859): t. cylindraceo-ovata; anfr. $7^{1 / 2}$, plani, ultim. $1 / 4$ longitudinis vix superans; apertura semicircularis; columella recta, non truncata, peristomate albo, brevissime reflexo; long. $5 \frac{1}{2}$, diam. $21 / 2 \mathrm{~mm}$. (Celafonia). T. subrimata, pallide corneo-fulva, sutura plana, linea alba marginata, apert. parvula, obliqua.

9. C. emiliana Ben. ap. Bourg. (1858): t. cylindracea; anfr. $71 / 2$, plani, ultimus $1 / 4$ longitudinis subrquans; apertura semirotundata, columella simplici, margine dextro in medio paullo incrassato, columellari in callo albido filiformi, adpresso; long. 9, diam. $2 \mathrm{~mm}$. (Sicitia.) T. cornea, spira conica, sutura vix immersa, apert. parum obliqua. Fig. Rev. Zool. 1858. t. 18. f. 1-3.

10. C. cylindracea CALC. (1840): t. cylindraceo-elongata; anfr. $6 \frac{1}{2}$, plani, uitimo penultimo multo majore, apertura lunato-oblonga, columella vix torto-plicata, marginibus separatis; long. 6, diam. $1^{3} / 4, \mathrm{~mm}$. (Sicilia.). T. crystallino-alba vel ad basin pallide cornea, sutura vix impressa, apert. parum obliqua, peristomate simplici, recto. Fig. Rev. Zool. 1858. t. 18. f. 7-9.

11. C. incerta Ben. ap. Bourg. (1858): t. cylindrico-oblonga; anfr. 7, convexiusculi, ultimo $1 / 3$ longitudinis æequante; apertura semioblonga, marginibus junctis, externo simplici, columellari in callo filiformi albido adpresso; long. 5, diam. $11 / 4 \mathrm{~mm}$. (Sicilia.) T. crystallino-cornea, sutura impressa, apertura fere verticalis, columella simplex, recta. Fig. Rev. Zool. 1858, t. 18. f. 15-17.

12. C. boissyi Dup. (1850): t. elongato-cylindrica; anfr. 6--7, subplanulati, "ultimus $1 / 2$ longit. saltem efformans;" apertura semirotundata, subpyriformis; long. 6, diam. 11/2 mm. (Pyrence Gallia.) T. subrimata, corneo-albida sutura tenuis, peristoma rectum, vix albido-labiatum, marginibus inæqualibus, callo distincto tenui filiformi unitis. Syn. Zua b. Dup., Bulim. subcylindricus $\zeta$ M. Tand. 1853, B. subcyl. v. fusiformis M. 'Tand. 1855 \& Ach. lubr. fusif. Picard 1840. Fig. Dup. Hist. t. 15. f. 9. Spec. a cl. Fagot communicatum anfr. ult. $1 / 3$ long. parum superantem cum figura citata omnino congruentem præbet.

13. C. dupuyana Bourg. ap. FAGot (1876): differt a C. boissyi testa lævissima, paullo majore, anfr. 6, subplanis, apice obtuso, apertura lunata (pariete penultimi ventroso-arcuato), se- 
mioblonga, columella oblique recta, margine externo convexo; long. 61/2, diam. $2 \mathrm{~mm}$. (Pyrencer, Gallice.)

3. Ferussacia (Risso) Bovici. Testa oblongo-etonguta vel sulfusiformis, lavis, nitida; anfr. planiusculi, in apiccm acutum terminati; apertura circiter dimidiam longitudinem aquans, columella torta, callosa, oblique truncata, paries aperturalis callo dilatato pertenui munitus.

Infbitat in silvis, hortis, fruticetis, in muscis, inter folia putrescentia et sub lapidibus, et precipue in alluviis fluviorum.

1.) Folliculiana BourG:: testa magna (l. -10, d. 3-4 mm.), corneo-lutescens, columella recta, intus callosa vel sublamellosa.

14. C. folliculus Gronov. (1781): t. cylindrico-elongata; anfi. 6, irregulariter crescentes, 4:s paullo major, 5:s magnus, ultimus $1 / 2$ long. non attingens; apert. lunato-oblonga, margine exteriore ad insertionem primo sulcato, antrorsum arcuato; long. 9, diam. $3 \mathrm{~mm}$. (Gallia merid.. Italia, Catalonia, Lusitania.) T. corneolutescens, spira conico-elongatula, peristoma acutum, simplex, colttmella parvula, recta. Fig. Bourg. Malac. chat. d'If. t. 2. f. 1-3.

15. C. regularis Boura. (1866): t. cylindrico-elongata; anfr. 7 , regulariter crescentes, ultimus $1 / 2$ longitudinis non attingens; apertura oblonga, margine externo antrorsum regulariter arcuatulo; long. 10, diam. 3 mm. (Ins. Mralta, Itul. ad Napoli.) T. corneo-lutescens, spira conico-elongata, peristoma rectum, simplex columella recta, parvula, parum intus torto-lamellosa. Fig. Bourg. l. c. t. 8. f. $7-9$.

16. C. nitidissima Kryn. (1833): t. ovato-oblonga; anfr. 6; apertura elongata, angulata, antice obtusa, columella labro duplo brevior, subcontorta, peristoma simplex, albescens; long. $4^{1}{ }_{2}$, diam. $1^{3} / 4$ ". (T'auria). T. acuminata, flavescenti-cornen. (Kr.) "An ealem species ac Ach. folliculus?" (Pfr.)

17. C. gronoviana Risso (1826): t. cylindrico-obesoque-elongata; anfr. 6, irregulariter accrescentes, tribus superis regularibus, 4:0 majore, sinistre præsertim convexo, 5:o maximo, ultimo $1 / 2$ longit. won attingente; apertura lunato-oblonga, margine exteriore parum ac regulariter antrorsum arcuato; long. 8, diam. $3^{1 / 4} \mathrm{~mm}$. (Galloprovincia, Italia, Malta, Sicilia, Dalm., ad La- 
croma, Gracia.) T. "paullulum streptaxiformis" (Bg.), pallide corneo-lutescens, spira attenuata, obtusa, peristoma recturn, simplex, columella paullo curvata, parvula, vix sublamellosa. Syn. Ach. folliculus Ben. Fig. Bourg. 1. c. t. 2. f. 4-6.

18. C. vescoi Bourt. (1856): t. oblonga; anfr. 6, supremi regulares, 5:us maxime accrescens, ultimus $2 / 5$ longit. rquans; apertura oblonga, margine dextro antrorsum arcuato; long. 9, diam. 4 mm. (Gallia, Hisp., Lusitan., Italia, Sicilia, Malta, Gracia.) T. cornen-lutescens, columella recta, intus calloso-contorta, ad basin attingens. Syn. Ach. palustris Parr. Fig. Bourg. 1. c. t. 2. f. 10-13. Forte varietas C. folliculi.

19. C. nebrodensis BeN. (1862): t. elongato-fusiformis; anfr. 7, regulariter accrescentes, ultimus cylindraceus, basi rotundatus, $2 / 5$ longitudinis subrquans; apertura semiovalis, superne angulata; long. $9^{1} / 2$, diam. $3 \mathrm{~mm}$. (Sicilia.) T. cornea, spira elongato-conica, apice rotundata, sutura impressa, anguste marginata. Fig. Ben. Ill. Sicil. t. 10. f. 6. (Ben.).

Obs. Celeb. Bourgurgnat in Malac. Alger. duas species C. vitrea Webb \& Berth. analogas nominavit: C. (Fér.) aphetina, "species spira perelongata, F. vitræ analoga" (in Sicilia) \& C. (Fér.) abromia, "species lanceolata, ut F. vitrea, costulata" (in Algeria et Sicilia). Descriptiones earum non vidi, nec specimina. C. vitrea W. \& B. sese, teste cel. Mousson, distingit: testa subcylindracea, spira celeriter attenuata, anfr. $6^{1} / 2-7$, primis regularibus, de tertio magis descendentibus, 4:0 et 5:0 elongatis, ultimo breviore, non attenuato, plane rotundato, apert. brevi, late pyriformi, superne angulata, inferne rotundata, perist. tenue labiato; $1.6{ }^{1} / 2-7$, d. $2 \mathrm{~mm}$. (Teneriffa, Lanzarote, Fuerteventura.)

2.) Proceruliana Bourg.: testa major (l. 13, d. $4^{1} / 2$ mm.), pariete aperturati albido-unicallosa, columella bicallosa.

20. C. laniellifera Mores. (1851): t. fusiformi - elongata; anfr. 7, supremi lente accrescentes, 5:us paullo major, ultimus $1 / 2$ longit. non æquans; apertura oblonga, margine externo sat arcuato, marginibus vallido callo junctis; long. 13 , diam. $4^{1 / 2} \mathrm{~mm}$. (Sicilia: Ben.) T. corneo-lutescens, spira elongato-conica, peristoma rectum, simplex, columella contorta, ad basin sicut truncata. Syn. Glandina l. Mor., Tornatellina l. Pfr. \& Azeca l. Pfr. olim. Fig. Ben. Ill. Sic. t. 5. f. 28. (Ben.)

3.) Tohenwartiana Boung.: testa minor (l. $4^{1} / 2=6^{1} / 2$, d. $2-2^{1 / 2}$ mm.), albida 
rel hyalino-vitracea, columella truncatula, curvata.

21. C. hohenwarti Rossxr. (1839): t. subfusiformi-oblonga; anfr. 6, nltimus convexiusculus, spiram non requans; columella vix truncatula, basin non attingens; apertura oblongo-acuminata, margine dextro medio subdilatato; long. $6^{1} / 2$, diam. $2^{1 / 2} \mathrm{~mm}$. (Carniolia, Ital. septentr., I'riest, Dalmatia.) T. lutescens, spira turrita, obtusa, anfr. convexiusculi, columella verticalis, intorta, apertura angusta. Syn. Helix pusilla Schacchi 1833? Fig. Rossm. Icon. f. 657.

22. C. jani De Betta (1855): t. fusiformis; anfr. 6, ultimus ventricosus, dimidium testæ æquans; columella arcuata, basi abrupte truncata; apert. pyriformi-elongata, margine dextro antrorsum valde dilatato; long. $51 / 2-63 / \%$, diam. $2-21 / 2 \mathrm{~mm}$. (Italia borealis, Tirolia merid.) T. albida, spira conica, acutiuscula, peristoma rectum, acutur. Syn. Ach. acicula Porro, aciculoides Strob., veneta Charp., Gredl. Fig. Bourg. in Rev. Zool. 1857. t. 1. f. 5, 6.

23. C. gredleri Küst. (1\$70): t. ovato-fusiformis; anfr. 6, ultimus $1 / 2$ longitudinis superans; columella breviter paullo arcuata, oblique truncata; apertura lanceolata, margine dextro antrorsum arcuato; long. $5 \frac{1}{2}$, diam. vix $2 \mathrm{~mm}$. (Tirolia ad Trient, Tricst.) T. albida, spira acutiuscula, anfr. convexiusculi, apertura angusta. Fig. Neunter Ber. nat. Ges. Bamb. 1870. t. 1.

24. C. bugesi Bourg. (1866): t. oblongo-elongata ; anfr. 6, duo supremi lente, cæeteri velociter crescentes, 5:us maximus, convexior, ultimus magnus, rapide descendens, $1 / 2$ longitudinis exacte requans; columella basin non attingens, subtruncatula; apertura pyriformis, margine externo antrorsum arcuato; long. 5, diam. $2 \mathrm{~mm}$. (Gall. prope Montpellier in alluviis fluvii Lez.) T. hyalino-vitracèa, spira sat elongata, obtusiuscula, apert. superne acute angulata. Fig. Bourg. Moll. nour, t. 30. f. 12-14. (Bg.).

25. C. paladilhi BourG. (1866): t, elongato-lanecolata: anfr. 7 , regulariter ac sat rapide accrescentes, ultimus $1 / 2$ longitudinis non attingens, paullo descendens; columella subtruncata, basin non attingens; apertura pyriformis, margine dextro antrorsum presertim ad partem basalem arcuato; long. 6, diam. $2 \mathrm{~mm}$. (Gall. cum priore.) T. hyalino-vitracea, spira producta, lanceolata, anfr. subeonvexiusculi, sutura impressa, apert. superne angulata. Fig. Bourg. 1. c. t. 30. f. $18-20$. (Bg.)

26. C. moitessieri Boura. (1866): t. oblonga; anfr. $6-7$, lente ac regulariter accrescentes, ultimus dilatatus, non descen- 





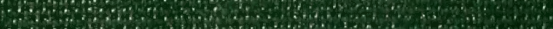

